

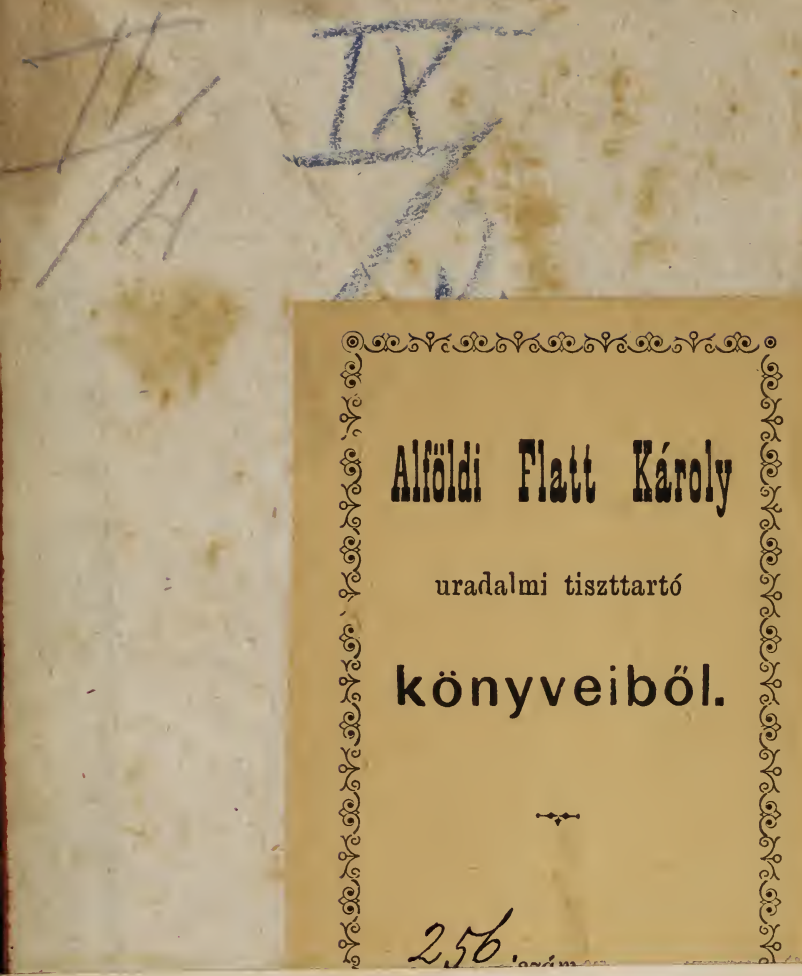

$\frac{43}{5 \times 4} 9.5$

1493
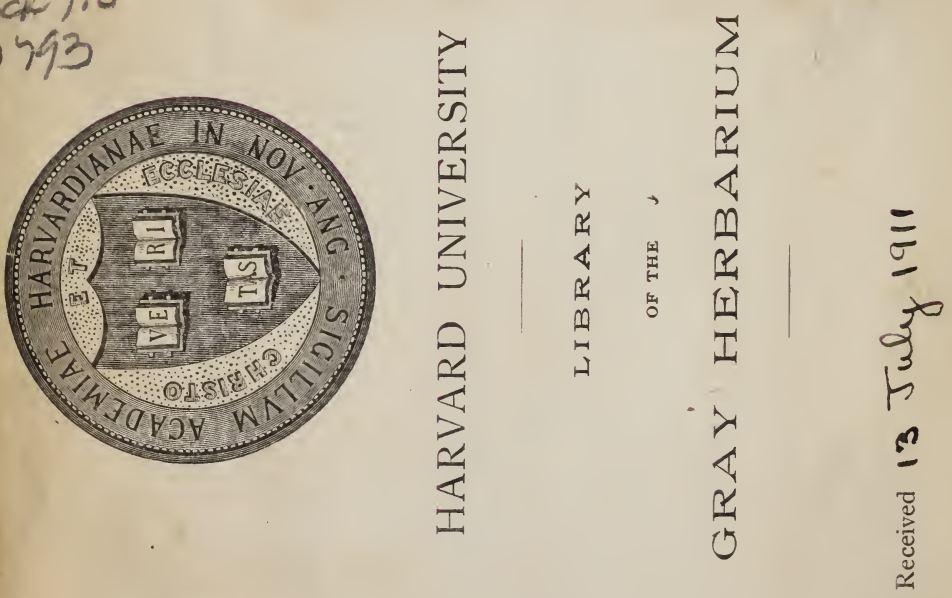




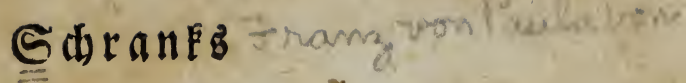

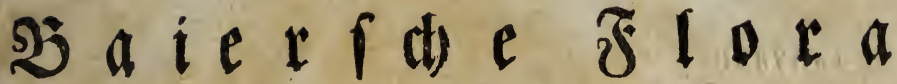

sum bequemern Gebrauche

als

S

in tabellarifale form gebract.

Rit einigen

Aenderungen un $\mathcal{Z}$ erbefierungen.

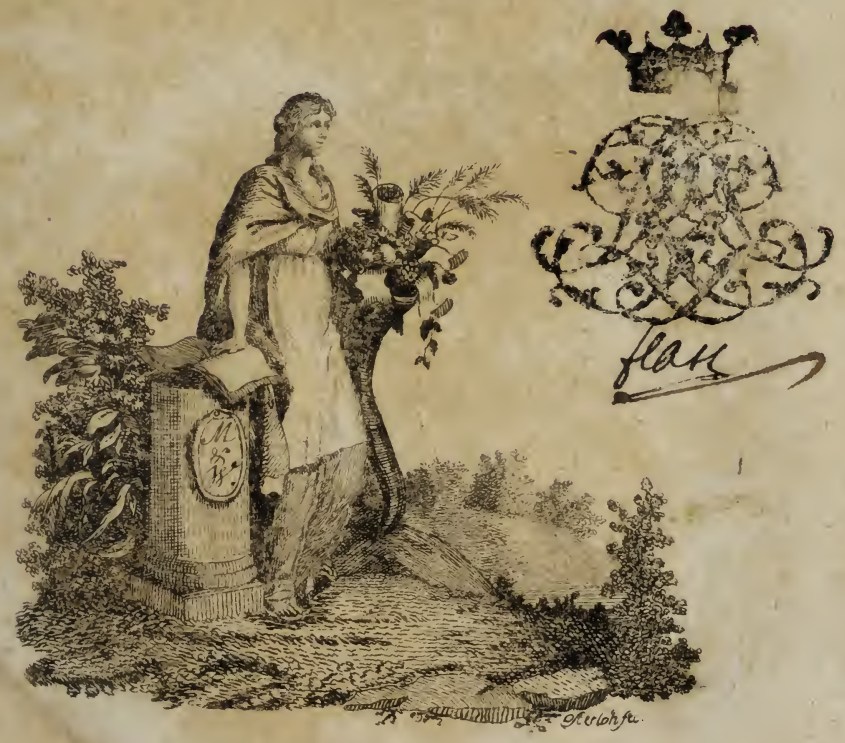

Tiegensburg,

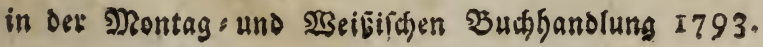


Out 13,1911

Gray Harbarlum Mervasd University 


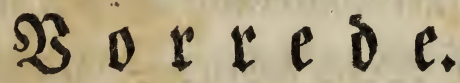

乃

Cirfts ift Dem 2ufuánger in Der $\mathfrak{B} 0$ tanif unents behrlicber, und felbft Dem geribtern Senner nidbtz angenehmer, als ein bequemes botanifforez Șands,

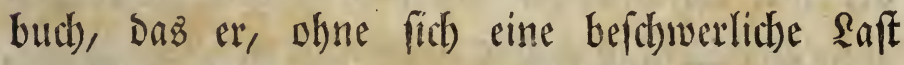
aufoulegen, auf peine Excurfiunen mitnelymen, und fogleich, auf Der Stelle ciner gefundenen \$Jflanze, zu Piathe ziefhen fann.

Sith glaubte, Dem Deutfichen, noch mehr bem baievfoten Soutanifer einen Beffallen zu erweifen, wemn ich) Dem $23 u n f(b)$, Den einige Sitteraturzeitunt gen áuß̧erten, entfprád)e, Schranfs gilora nad)

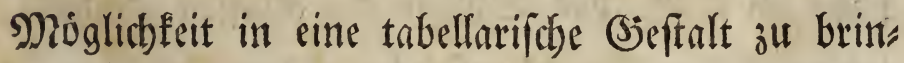
gen, wie ez einft Schraber mit einer betráctytlis

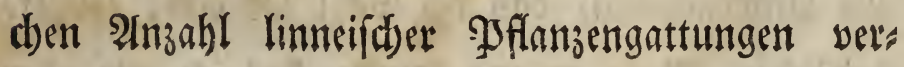
futbte. 
Sibranks baierfbe Flora fobint mir vor andern zu fo cinem Sandoutche tauglicí) zu feun; weil beffen naturbiftorifbe Werfe bereits̃ cinen entidbiebenen 2 Berth baben; - weil biefe Flora fo mantbe Beritbtigungen und Berbefferungen bes linneiften Syftemes enthált; - weil fie fü bair erfche Botanifer gerade Dab erfte, witbtigfte $2 B e r f$ ift; - weil fie gewifis manther Siráterfundis get als Safobenbuch mit fich tragen zu fornnen swünft); - und weil fie fith endlich ofne grofent

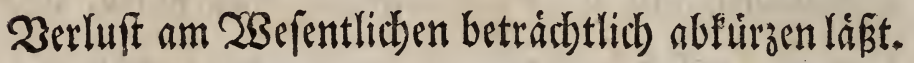

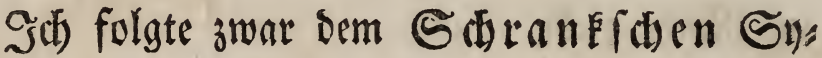
fteme mit vieler (bienauigfeit, bod) erlaubte ids mir auch einige (abweidbungen, wenn idh Dadurdh Die Sache Deutlicber vorzuftellen, ober Die Muibe Dez Foricherz zu verringern hoffen fonnte. So babe ich j. B. Daz Syftem Der Rlaffen geándert,

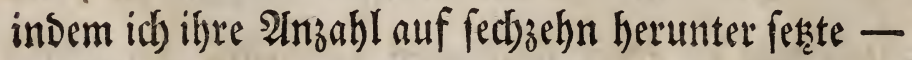
ein UInternebmen, bab ich wohl bely einer fonders beitlichen flora wagen fonnte, bas idh mir aber 
bey einem vollitindigen Syfteme nicht fo leicht erlauben wurde. - So babe ich ferner Der Bes

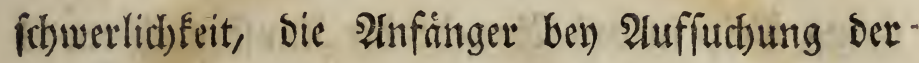
PSflangen mit ganz getrennten (Siefd)ledbtern fins Den, badurtb abzuhelfen geglaubt, Daf́ itb bie ganz månnlicben Bluthen, ganz für fid alleine, obne

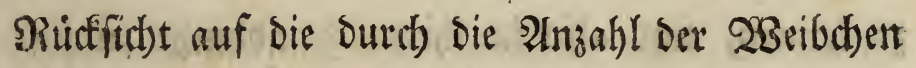
beftimmen Sronungen, und eben fo auth bie weiblichen 3 luthen, ohne Rutefitht auf bie surch bie Maannd)en beftimmte Slaffe, in swoen Dros nungen einer neuen Slaffe, wovon bie erfte alle blos mánnlicten, bie swote alle blos weiblichen Blithen enthålt, behandelte. - So habe id auch), nact) bem Beyfpiele Sude ows, bie vors zuglidfften Dronungen jeber Rlaffe, wo es bie Natur ser itiol felber enthaltenen \$ुflanzengattun: gen geftattete, Dureb bie Pbtheilung in Baiume, Stauben, Rráuter uno (Soráfer ic. gemacht, weil mich (Erfabrung uberzeugt, baj burd) Diefe, jebem auffallende, uno nut in wenigen Făls Ien triglidye Eintbeilung fehr viele Dhube uno

$$
\mathfrak{A}_{3} \quad \text { Beit }
$$


Beit beym Ltnterfuchungagefóafte erjparet werde, und weil idb $\mathfrak{e z}$ fir eine fehr verzeibliche 2 bweis (d)ung hielt, in bas (Siewebe ber אunft aud) etwab Natur mit cinzutragen. - So nahm id) endlich auch) bie allgemeinern (Sartengewaicb) auf,

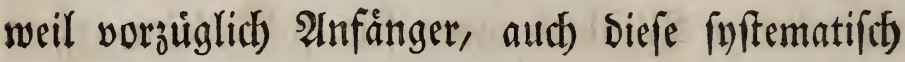
zu fennen, und alfo in ifrem Sandbutbe zu fint Den wunffben.

Da $\mathfrak{e}^{b}$, wenn mant cinmal Den Siattungas: namen einer P̧flanje gefunden hat, fehr vergebliche Mhihe und wahke Beitverfplitterung ift, bie for on befannte (Siattung, um audb ibre Arten zu finden, nochmal nach Slaffen und Sronungen, und ber meiftens nur foseinbaren, oft mit vielem Zwange aufgeftellten Berwandtfdaft Derfelben, fuchen zu múken: fo bractete ich die (jattungsnamen in sin alphabetifchez Riegifter, wo Dann bey ieder (Szats tung bie verichiebenen befannten 2 rten, eben aud) wieber, to viel ez muglid war, in tabellarifthe Form gebractht fint. 
Den lateinifichen (B)nttungbnamen muste it)

bey Diefem Siegifter Dem Deutf(t)en vorziehen, weil er beftimmter ift, uns weil audb ein bloz beut: forer Naturforfither Den im Syfteme vor fidh ftehenden lateinifiben 9Ramen ohne Seftawerde im Regifter wiro nadbidhlagen fónnen. Llm Der Rlage liber bie Befchwerlichleit, die bie vielen barbarifhen ober halbgriedsifhen Woirter bent (biebátbtnife Der Nichtlateiner verurfacben, aแรzus weithen, habe idh auch Die Deutifhen Benennungen beygejesct.

Die Zerbefferungen, bie id) in litterarifiben Seitungen, Sournalen 2 . angezeigt fano, bielt ich fur Pfflid)t anjubringen, fo bald idf mich) riberzets gen fonnte, Daß̧ fie Berbefferungen wairen.

Dic Angeige Des 2 Bohnortes beftimmte iđh) lieber nach Der sage, und Der Befdaffenbeit des Sodens überhauptz, alz nach indiviouelen Sort. f(b)aften. Sob idh hierim redit gethan babe, mo: gen Freunde Der Botanif entifteiden.

$$
\mathfrak{I}_{4} \text { Den }
$$




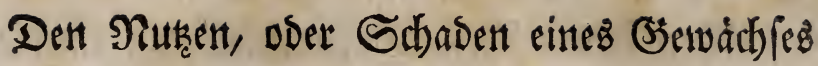

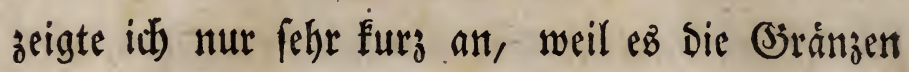

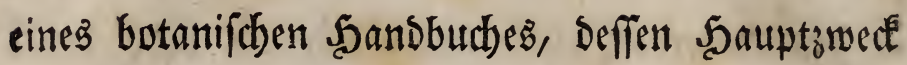

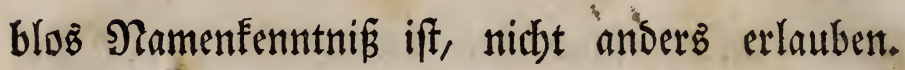
(J)a nidbtz bawon zu melden, bielt id für einen Febler, weil immer bie erfte Frage bey einer neu befannt gemachten $\mathfrak{P f l a n z e}$ ift: " $\mathfrak{B a z}$ bat fie "für Eigenldhaften? was nutht. fie?"

Die mit einem * bezeichnetent fint in ber פachbarfchaft Der Sauptftadt $\mathfrak{B a i e r n z ~ g e f u n d e n ~}$ worden.

Shabe id) Durch Diefe fleine Bemůhung Siråus terfreunden Etwaz angenebmez geleiftet, fo madjt es mir wahre Freude. 


\section{Ibeil. Battungen.}

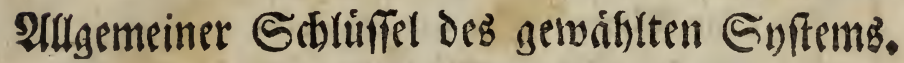

A. ewaedie mit beutlidben Shluthentbeilen.

2. 3witter.

a Uluverbutbene Staubgefáze, uno zwar

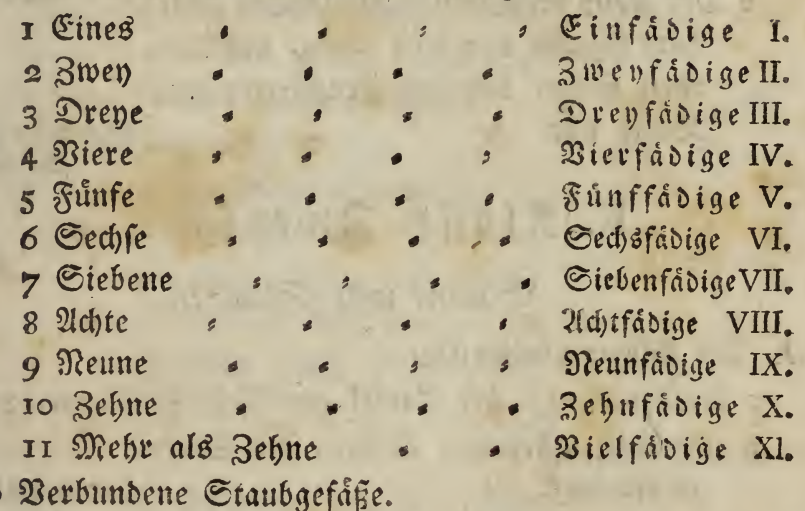

aa )ittelft Der Triger.

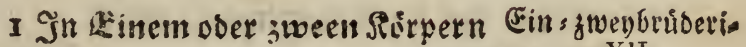

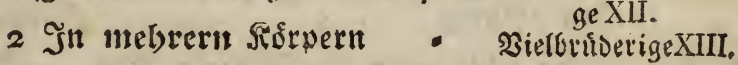

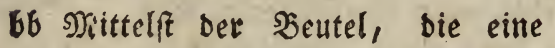

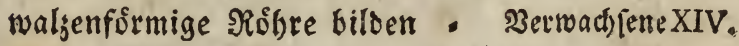

25. Эlos mânnlidje, ober blos weiblidje Sllitben

.

B. Berwåcbie mit unbeutlichen \$lutthetbei len

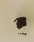

Bjetrennte Bies (ib)lechts = Ṡlutthen $\mathrm{XV}$.

Unfenntliçe গ̧lú: then XVI. 


\section{Rlaffe. Sinfádige.}

Siratut.

A. Mit einem Staubroge: Der Seld febr furg; bie Slume feblt, Det Staubfa. Den fitht auf Dem Fruchtenoten. Der Saame nut Ein Sorn " Edjafthalm, Hip-

B. Nit zmeen Staubwegen. puris.

a Der Relch feblt; bie Sllume zwenbläts terig; Die fiapfel zwevfäd)erig, vier. faamig

$=$

b Der sield orentheilig; die Slume feblt; গBafferfe:u, Calber Sname nur Ein Rorn, bie blüs then fellen bey ber Frudtitreife eine Seere vor = : Sthminfuect, Blitum.

\section{Rlafie. Sweyfidige.}

\section{Şåume uno Stauten.}

A. Die Slume vierfpaltig,

a tridterfórmig; bie Rapfel swenfádherig Stieder, Syringa.

b mit malgenformiger Siơbre; Die Љeere vierfaamig = " 5ૃartriegel, Ligu-

B. Die Slume ungleich, mit smenfpaltiger litriche. 
2. Die Slume rachenformig, gefpornt; Die Sapfel ifád)erig

I Der Reldh faft zweylippig, frinfs ipaltig

Fettkraut, Pinguicula.

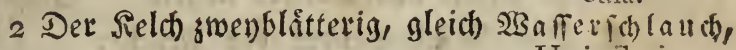

5. Die Slume rabformig, 4-5fpaltig; Utricularia. ein Etude Eleiner; Der Sielch 4-5 theilig; Rapiel zfacherig, berzfơ: mig

๔. Die Sllume einlippig, einblatterig, EFrenpretia, Veronica. bie Rippe ungetheilt. Nit cinem Saulden, bas eine balbtellerfor: mige शarbe, unb zween Staubbeus

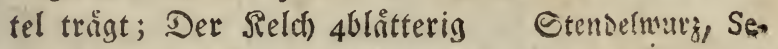

D. Die Slume slippis.

A. Der Shlutbenftanb beutellos.

a Die Sobrlippe fefr furz, ber Sield) fünflatterig gefơrbt, bie Etmugefåfe nufitebment ; bie Unterlippe grofi, wegftebend, flack);

$x$ fpornlos , : Tagrour, Epipa-

2 rúcfwairts in ein f̧orn verlängert = Snabentraut, Or-

33 Die Sberlippe flein; Die rapias.

Ilnterlippe felgr grofi, boot: formig, allfgeblafen; ber Sield) 6blâtterig, gefärbt; bie Staubfáben all die Sh: ren bes Selmes anges wachen, burdh èine Forts rek̨ung mit bem Etengel nerbunden

- 2ienus[ifuli, Cypripedium.

b Nier 
b Nier nacte Saamen; ber Selch gleidffirmig; bie Slume 4fpals tig, faft regelmåßigi, ein \&ap. pen auggeranbet; bie Staubs. gefễ

B. Die Sluithen ober ber frucht; pus.

Sielch) und Sllume 2blatterig; bie frudft fteifborftig, meiftens 2faa: mig

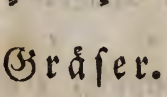

Jૃextenfinut, Circaea.

* Staubwege; Der Seld ein 2/dualiges, Iblütbiges Эålglein; bie Slume eine 2idhaalige Spelige

s
Rutigras, Anthoxanthum.

\section{Riaffe. Drebfádige.}

\section{Siduter.}

A. Nit I Stautwege.

2. Die Slüthen uீber ber Frutht.

a Der Rield feblt; bie Slume einblät. terig, an Brunbe gebogen; Der Saa: men nut Gin Forn. - "Sbaldrian, Vale-

b Die Sllume 6theilig; riana.

a gleids; Der Biriffel bat 3 sufamm. gerollte Narben

Safran, Crocus.

5 Die bren obern Slumenftucfé fteben naifer aneinander, bie untern mebr weg; bie Staubfäben fommen aus ben Winfeln ber Slumenftúffe, aufitebeno

Sdjwerditie, Gla-

e in ber Mitte, brenblåtterig, bie diolus. Slútbe umgefebrt; bie Staubbeu: tel auşwårts gefebrt

Sdjwettel, Iris. 
2. Die Bluthe unter Der Frudt, ber Rel 3 blätterig; Die Şlume 5 blâtterig; Der Gaamen nur Ein Rorn, ifhnedtenfor: mig

B. Mit 3 Etaubrogen.

Snorpelfraut, Polycnemum.

a Der Stelá) 2blätterig; Die Slume eins blätterig, unregelinn̊fig; bie Sapfel bren: faninig

b Selch und Sllume 5 blåtterig; bie SRap: fel einfẩberig, faft walzenfơrmig, an Der Epitae fich o̊fnent Siontie, Montia.

A. Mit I Staubmege.

\section{(5) id fer.}

2. Der Etengel mit einer marfigen Sub: ftanz gefüllt; (Şalbgras) bie Sllume feblt; Der Caame einfórnig; bie Rielch= bålglein fpreuartig.

a gefåuft, einfchaalig . = Rnopfgras, Schoe-

$\mathrm{b}$ auf zwo Geiten, Dachsiegelformig Eupergras; Cypec unordentlich Dactziegelforrmig. rus,

a Die sluttben in faft waljenfors. migen Alebrcten; Der Saamen nacfit ", " = Binien, Scirpus.

b Nertrodfnet; ber Saame mit fefr langer Seibe umgeben. 2230llgras, Erio-

55. Der Stengel (Scalm) hobl; Der Relth phorum.

feflt; bie Slume eine zmevidhalige

Epelge = $=$ " : Borftengras, Nar.

B. Mit 2 Etaubregen. dus.

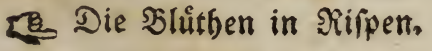

2. Der Selch einblưthig

2 zmeyflappig

a Die slume einfaliefents 
1 Die Stlappen fabufürnig, gleichlang = - Słlanjgrag, Phala-

2 gleidfbreit, oben runb auss, ris. geidnitten, mit boppelter Epise

Qiejograb,Phleum.

b Die Slappen faft gleid)grof; Die Spelge felre futry; Die Rar: ben pinfelformig . STritengrab, Mi-

c Die Jilappen ipitaig, fleiner, als lium. Die Epelze; Die Natben Det fainge nad) rauth

D Die Epelse bat an "ber ånęern Straubarab, Agroftis.

Selappe eine gerwumbene, am Grunde gegliederte, auperor: Dentlich lange Granne = Pfriemengras,

e Die Spelje cinflappig, mit ein= facher Spise " = Fuchofiofmanz,Alo-

b Drevtlappig, Die Dritte Silappe fưrjer " " " Ediwaden, Pani-

3. Der Reld) zblúthig, zmenflappig.

a Mit einem britten, nod) unent= wickelten Slüthd)en; bie $\mathfrak{J l u t t h =}$ d)ell vom Feldhe eingebuillet, bie Relchflappen gleid) " = Jierlguab, Melica. b Drne cinem oritten Sllutthchen Sirnelen, Aira.

c Die ânfere Relchlappe grôfer, Die fleinere umfä̧end; Die åll fere Spelze meiftens mit einer Siranne 3 s Dferograb, Hol-

(C. Dex Sield) 2 biz zblutthig, furget, cus. alB Die Slúthdhen, zllappig; bie silappen ungleid), langetfơrmig, fes gelforrmig ineinander gerollet; bie \{ifpen Dunfel veilblau

- Molinie, Molinia. 
D. Der Reld vielbluthig, zmenflappis;

a Die Silappen am Sande vertrodf= net, etwas fpitigig; Die Alebrchen eyformig =" = Silpengrab, Poa.

b Die Silappen bauthis; bie Sllume berzfơrmin; bie Siipenåfe libers baingend mit zitternben 2 eforden Bittergraz, Briza.

c bie eine Rlappe gróßer, mit ei=

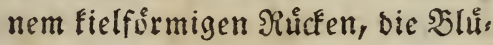

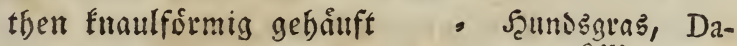
d Die Rebrchen rundicht mit einer ctilis.

Granne alts der Epitze Der zwey)= flappigen Epelge = = Eiswingel, Fe-

e Die Aebrchen linglidft, Die Giran, ftuca. nen unter Der Epitze der Epels zenflappen; Die Epeljen zjeilig Trefp, Bromus.

f Die 2lebrchen långlicht; eine ge=

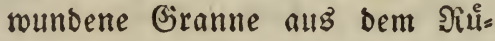
cfen, ober alts bem Grumbe = Şaber, Avena.

g Die Spelize unbemegrt, am Grun: De zottig; Die Sieldhlappen gleidh Robr, Arundo.

2. Die Slluthen in 2tebren.

a Der Sielch Iflappig, vielblütbig; bie Rlappen an Der Alukenfeite. Die Aebrchen flachgebruidft, mit ber fla: d)en Seite an bie gemeinichaftlidje Spule angebrưcft " " Sold, Lolium.

b Der Seldh 2flappig.

a Iblưthig,

I an Der Seite ber Shlúthathen; allezeit 3 \$lüthchen in 6 sield): flappen beyfammen (mad)en bas fect) $\mathfrak{b}$ lâtterige $3 \mathfrak{b l u m i g e}$

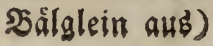

(3etfe, Hordeum. 
2 Die Slůtbchen eingeln; bie Granne gegliebert s Sartgras,Andro-

b zblutbig, Die imneve Rlappe an pogon.

bie gemeintohaftliche Spule ange. oruckt $=\quad$ : Roggen, Secale.

c Sielbluthig;

I bie Saanten långlid)t enfo̊rmig, bevberfeitz frumpf; Der Eiruno ber Epule frumpf $\quad$ weizen,Triticum.

2 Die zweyblattrigen Rlappen an Den breiten Geiten Der flad) gebruidten $\mathfrak{A}$ ebrdben, bie mit Der flad)en Seite an bie ge: meinf(j)aftlidise Epule anges Sruicft fino " Scaatgras, Ely-

3 Die Sullappen an ben falmalen mus.

Seiten, bie innert febre furg; bie Alebrdsen flach gebruidft, - mit ber formalen. Seite an bie Spule angelebnt $=$ = Tinuldiglas,Crae-

4 Die filappen linienformig zuge: palia.

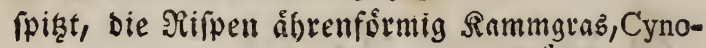
fiurus.

\section{RIaffe. Bierfadige.}

Die Graubgefâpe mit ber Blume nid)t verwadhren.

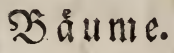

Mit I Staubrese, ber Reld) 4 afjnig, feft furz; sie \$llume 4 blåtterig, ůber bent Fruthtfnoten; bie Stcinfrudjt cine 2f̊̊: d)erige \$?แี

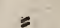
Siñ ter.

1. Mit I Staubrwege

A. Die Slutben ůber Der Frudbt

a Die Slume einblátterig 
a trichterformig; bie zween Samen

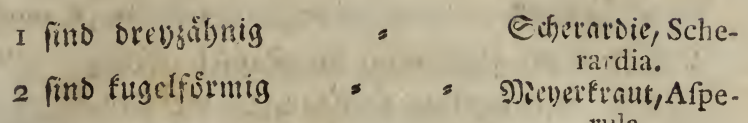

6 rabfurmig; bie zmeen Saamen furt

fugelfórmig = = Eabtraut, Ga-

b Die SIlume 4tlleilig

lium.

I Der Relch 2blatterig; bie Rapiel

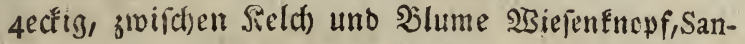

\& Der Seld́ Eaum Eennbar; die Siap. guiforba. fel lederartig, jufammgedruidt jus rưffofichlageli, = = \$alantie; Valan-

c Sie Silume 4blatterig, ber Sield tia.

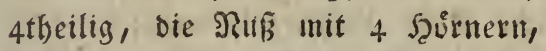
Den ebemabligen Feldtheilen = Stadielnú,

B. Die sluthen unter ber Frud)t.

Trapa.

a Sield uno slume 4fwaltig; Der Sans Det Sllume ungebogen; Die Etaub. triger fefr lang, die Rapjel afäches rig s

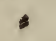
- Sisegerid), Plan-

b Die \$llume fellt; bet Gaame im rieldie eingefdilofien.

I Der Sield) sipaltig, ber Saas

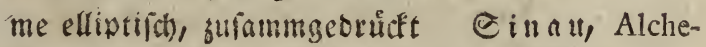

2 Der Reld) 4fpaltig; Der Sans me enforrmig milla.

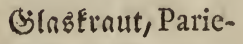

II. Nit 2 Etaubroegett. taria.

a Der field) 4 oder spaltig; bie Silume feblt, I oder 2 Saamen " "Fiauenmintel. b Der Seld 4 biŝ 5 theilig; bie Slume dyen, Aphanes. 4 bis 5 fraltig, bie Siapfel afaid)erig Fladjgleive, CuIII. Nit 4 Ctaubregen; Die slume 4 blats terig. 
a Ser Selds feglt; ber Briffel feblt, 4 Sanmen = - " Enameraut, Po-

b Der Sielch 4blítterig, bie Stapiel rfáches tamogeton. rig, vierfhanlig, vielfaanig; bie Tră.

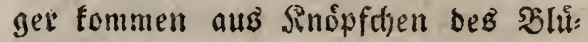
thebodens $==+1=$ Miafteraut,

Die Etaubgefäpe mit our Shlume verwad)[en. Síduter.

I. Nit I nacten Eaamen; ber Blumenbos ben foreutig;

a Die Slutthe unter ber frucht; ber ges meinf(d)aftlid)e Reld) Dad)jiegelfirmig; ber fonberfeitliche rôfrig; bie Slume bat eine atheilige Ober = uns eine 3 thei. lige Unterlippe

Sugetbrume, $\mathrm{Gl}$.

b Die Dluthe ober bem fruchtfnoten; bularia.

ber gemeinidhaftlidfe rield vielolatte. rig.

a Die Slume 4ffaltig; Der Fructsts boden enfórmig = = Sartendiftel,Dip-

- Die Sllume 4 ober 5fpaltig; ber facus. fonderbeitlid)e Reld) Doppelt; ber Saame mit einer Rrone "Scatiofe, Scabio-

II. Mit 4 maditen Saamen. fa.

A. Der Relch alippig.

2 Volffommen gans an ber Minbung, nach abgefallner Slume won ber obern Decielformigen Rippe gefdloffen

b $23 i e$ vertrodfnet, oben etwas fiad), Edjildéraut, Scubie Dberlippe etrab alf wårtz gebo: sen; bie Dberlippe Der ß̧lume et 
mas gemoltbt, 2ipaltig, an ber lins terlippe das Mittelftuck berffirmig Meliffe, Meliffa.

c Bilockenfórmig; die Dberlippe aus: gerduntten, bie untere 2fpaltig; bie Dberlippe ber Slume flad), bie uns tere 3fpaltig, ftumpf; bas, Mittels ftuck gróser, geferbt

- Millittis, Melit-

d Doer fůnf̧̧ånnig, ober 2blåtterig; tis. unter jebem Sieldhe ein braunes Sllatt; Diếe ஒlắttchen liegen wie Dad)giegel, und bilden eine itbrell. fơrmige frŭlle; bie Đberlippe ber Blume lo̊ffelformig, aufrecht, bie un. tere 3 ipaltig, bie Stucte enforrmig, gans

e Die Dberlippe Der Şlume belmfór. T:nioran; Origamig, zufammgedrucft, ausgeranbet, Die Unterlippe 3ipaltig; 4 Staubtrís ger, panrweife auf einem gemeins fdhaftlid)en Stiele; bie untern met: ftens beutellos.

- Calbevi, Salvia.

f Die Dofrlippe zzånnig, bie untere Sippe 2borftig; Der Ed)lund mit Zot: ten geid)loffen, zut frud)treife ver: engert

g Die Dberlippe ift tellerforrmig, bie , Thymian, Thyuntere 4 fpaltig; bie $\mathfrak{b l u m e ~ u m g e b o . ~}$ gen; bie beyben äufern Gtaubräden baben unten eiren umgebognen Fort. fât : : " :" Safilien, Ocy-

$\mathrm{h}$ Die Staubtraiger alle zmenpipizig, Der Staubbeutel an Der einen Spitze

- Dorunefle, Prunella. 
i Die Dberlippe 3 - bie mtere $2 \xi a ̊ b=$

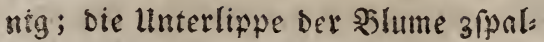
sig, oas snittelftuc 2theilig; unter den Duiuln eine vielborltige Sculle Sintrboften, Cli-

B. Der Feld in 5 ungleidse Abronnitte nopodium. geîalten, bie Slume Ilippig, rachens formig,

a in ber Rogre Gantis, bie Dber: lippecingefpaltenes 3åknd)en, das Dittelftucf ier unterlippe vers Eebrt bergformig \& $\quad$, Cunfel, Aiuga.

15 fratt Der Sobulippe bis an bie Siofre gefpalten, auscinander ftes beno; die Strubfioen an biefer Sboilung

C. Det Reld 5ådnig.

- Bamander. Teu. crium.

\section{Die $\mathfrak{3 l u m e ~} 2$ lippig}

2. Die Unterlippe 3fpaltig

a Die Drertippe belmfirmig, aa an ber Spire geterbt,

a Die Seitenfticte Der Itnter. lippe fpitzig, langetformig, zubenoen Eeiten bes Echlut= Des ein flacher 3ahn "Pollibie, Polli,

BDie Eeitenftucte Der Inter. chia.

lippe faft zugeruntet; $\mathfrak{z}$ bevs Den Seiten des Sdylunbes cill unten bobler $3 a b n$ \& Rogenoeficht, Ga. bb oft ausgefdintten, enforemig; leopfis. die Seitenftúffe Der unterlinpe abroints aeridtet; ser Echluno obne Zabn; Die yerblutgen 


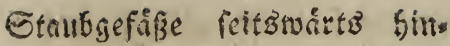

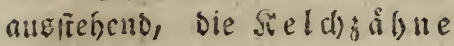

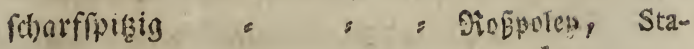

6 Die Oberlippe nemig gensift; chys. ber freld becherformig, 5ectig, Ioftreifig - Sallote, Ballota

c Die Doerlippe aufredst,

I bie Ctaubbeutel parmets in Beffalt eines fireujes zufams mengeneigt : = B̧unoetrebe, Gle-

2. Die eine Narbe bưllt Die ans coma.

Dere faft ein, Die Etaubgefábe

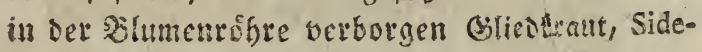

d Die Sberlippe flad); die Sisfre risis. gebogen, bie Relchzăhne graunen: formig

פ. Die Iliterlippe zlappig, werfelint Setonie, Betonica. berjfirmig, Die Dberlippe helmfis: mig; z̆l beiben Eeiten beb Edhluns bes ein flacher 3afn

- Taubneter, La-

c. Die Uluterlippe geferbt; Der Sias mium.

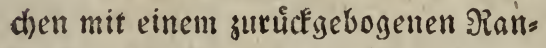
Se, sie Staubgefäße gemåbert : Nepte, Nepeta.

造: Die Slume faft regelmáping

a vieripaltig; bas breitere Stuct ausgeranbet; Die Eraubgefåfe aufred)t, auseinanber febeno = Narnge, Mentha.

b trichterformig, frumm; 2 soer 4 Etaubgefåfe; Samen po viel alB Staubgefåke; cin $3 a \mathfrak{n}$ am sielche furger

Eijenfrant, Ver-. pena. 
D. Der Feld 5 auth mebr zingitg, bie Slume zlippig, bie Unterlippe 3ipaltig.

a Die Sberlippe gemólbt, ganz; bie Staubbeutel zumeilen mit glån. zenoen \$uncten befäet

Edwenturuang,

b Die Sberlippe gerabe, linienfors Leonurus. mig, 2paltig, ber Reld) tridter: firtemig, Ioftreifig

2fnoorn, Marrubium.

II. Mit bebedten Gaamen.

A. Der Selch ztwenlappig, ausgerandet, gefôtbt; bie Şlume slippig, meniger gefârbt; bie Dberlippe lănger (Rapfel 2föcherig)

- Vartfie, Bartfia.

B. Der Reldh 4fipaltig, bie Blume slippig,

a trid)terforrmig; bie Oberlippe 2 - bie untere 3 paltig; bie siapiel Ifáthe. rig, Ifaninig, fugelformig, vom Siels d)e eingehulllet; ber Steld) furz, re: gelmåß่̧

$=$ : Tozzie, Tozzia.

b frazengefictiähnlich

a Die Siapfel 2fåd)erig; zufammges bruicft.

I Die Staubbeutel 2lappis, eis ner ber untern bat an bem einen Lappen einen fleinen Stachel = " : 2lugentroft, Eu-

2 Die Slume radienformig, phrafia. bie Dberlippe zufanmges brictit, am Ranbe suruidfges fhlagen; bie Rapjel auf ber einen :Rath aufipringend Rubweizen, Melampyrum. 
3 Der Seld) baudbig, Jufamm. gebrickt, Die Siapfel frumpf Saghnenfam, Rhi-

6 Die Srapfel Ifåcherig; am Sirunbe nanthus. Des Fructifnotens ift eine ger bructle Druffe, bie Rarbe einfach Sinuppentour

C. Der Reld 5ipaltig. Lathraea.

a Die Slume elippig; bie Unterlippe 3theilig.

a Die Dberlippe gemsilbt.

I 2 m Grumse DeB Fcudtefno: tens ift eitre Srüle; bie Narbe etheilig; bie Siapiel einfo̊d)erig : = Connenwulz,Oro-

2 Die Siapfel 2ficherig, viel= banche. raamig; fpringt oben auf Eánleftaut, Pedi-

bie Soerlippe ftumpf, einges cularis. (d)nitten; bie Unterlippe febr fur; ; ber @ediluno offell, fugels forrnig aufgetrieben

כraunwurz, Scro-

c Die Dberlippe an ben Eeiten phularia. zurúcfigeidlagner, ber Edjluno burd) eitten Graumen gefdilors fen; ant Sirunbe mit einem Sporne, ober furgen Sacke; Eswenmal, An-

b Die Şlume glodenforrmig; die Sap: tirrhinum. fel vieliaamig.

I jmenfähdherig; bie গ̉lume balls

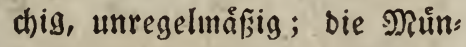
oung reicht, sfpaltig Singethut, Digi-

2 einfád)erig, 2fdaalig; bie ઝ̧lı. talis. me ziemlid) regelmẩig; bie Staubgefăfie paarneife genc์ bert

Simojelle, Limo. fella. 
D. Det Selch 5theilig, oder auch 7 blåt. terig, 2 Etrubfäben fino beutellog, (bie sapfel zwenficheris, 2fohaalig, viel. (aamig)

\section{Tinffridige.}

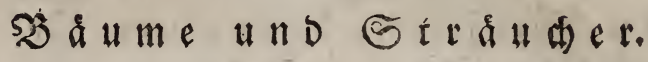 \\ Mit I Gtaubweg.}

A. Dic Dlume cinblatterig.

a Unter bem Frud,fnoten, flein, an Siel che angemadjen; Der Sians 4-5fpál= tig; (biere Itbichnitte foheinen blos Relchid)uppen

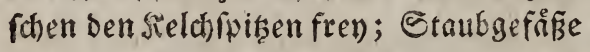
find fo viel als sllumenlappen; und ftehen biefen gegemiber; bie beere hat nicht to viele Fåd)er, als bie Slume Sappen; ber Seld) Iblâtterig, glodiens formig

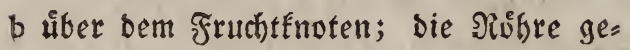
Sueuzoorn, Rham bogen, Der Rand 5 theilig, Das 5 te Stuice tiefer abgerondert, Der field) fefr Klein; bie $\mathfrak{b e r e} 2$ fächerig - Epedflitie, Loni.

B. Die Slume 4-5blatterig, unter Dem nus.

Frudtenoten, Der Field) 4-5theilig; Die Rapél 4-5fächerig, 4-5idhanlig, 4-5eckig, gefârbt

C. Die Blume 5 blătterig cera. Gratiola. 
b Slumenble̊ter แmo Etaubgefåge aus Dem Sitheooben; die Becre von Fieldye umgeben, sfaanig

Epbet, Hedera.

b Hnter Dem Frudtfnoten; Die Sllimen: blätter oben zufammentaugend; bet Briffel feblt; Die Deere 5 framig.

2seinftor, Vitis,

\section{Mit 2 Craubuegen.}

Der Reld 4-5fpaltig, bie Blume follt ; sie Sapiel ift eine bairtige, faftlofe, breitgeorudfe Slafe, oben mit citem (Einfd)nitte.

\section{Mit 3 Gtaubwigen.}

2 Die Sllume ưber Dem Frudtflnoten, funftheilig; Der Neld 5tbeilig.

a Die Beere eimfanmig,

জืatherbutien, Viburnum.

b - _ Dlevfonmig = Scotoct,Sambucus.

b Die Blume Imter Dem Funthoten, 5blitterig; Der Reld) 5theilig, ges färbt; bie 3 Rapfeln bertwachjen, aufgeblafen; in jeber jween fugels formige Snamen mit einer Sarbe. (S)t ift cine Sîpfel leer)

Wimpermuß, Stapliylea.

9) 4 Etaubwegen.

Die Sblume 5 blatterig.

Stedpalme, Tlex.

$$
\begin{aligned}
& \hat{\imath} \mathfrak{r} \mathfrak{a} \mathfrak{t} \in \mathfrak{r} \text {. } \\
& \text { כwoitter. } \\
& \text { Mit I Gta } \operatorname{s} \text { b wege. }
\end{aligned}
$$

I. Die Slumen I blåtterig.

A. Unter Dem Frudtifnoten;

24 nackte Saamen; 
a bet Schluno offen, fitralenlos, (nackt)

I jebes paar Caamen in eine be: fonvere Saamenfaut verfalof. ren; Der Sllumenrano robrig, aufgeblajen;

- \$23actistlume, Ce.

2. Die Slume glocfenförnig, un= gleich

rinthe.

3 Die Slume tridhterformig

aa Der Selch 5 fantig , Eungentraut, Pul-

b6 Der Seld 5theilig : monaria.

Eteininame, Lithofpermum.

b Der Salund der slume gefdlofien, und zwar

aa mit beroorragenben Edhuppdjen, Drůfen, ober budkeln

2a Die Slume práfentirtellers fớrmig, 5fpaltig; bie lappen auṡgeranbet $\quad=\quad=$ : Bergişmeinniçt,

bb Die Slume trichterfo̊rmig Myofotis.

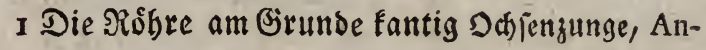

2 Die Robre ber Slume ges bogen = = = 2 Bolfägefítd)t, Lyc-

3 Die 4 Saamen mit eingel, opfis. nen, an bem Briffel anges befteten, (d) wadiftadhlichen

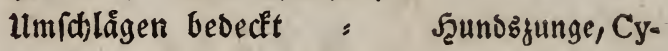

4. Der Frudtiteld) zulammges nogloffum. brưdt'; bie Saamen långs lid)t, sufainm, und aneinall.

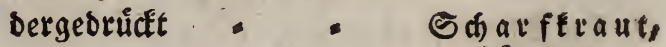
Afperugo.

cc Die Slume rabformig - Borretid, Borrago.

66 mit 
bb mit 5 pfriemenförnigen Spitzen, Die in Sufummneigen einen füegel bilben, ber slumenrand roobrig aufs geblaifen

b Die Eamen bebedf.

Seinmell, Sym. phytum,

aa in einer siapfel, bie

a Ifächerig ift

aa Die $\mathfrak{b l u m e n}$ in einer einfachen

Dolbe, die einen Hinfthlag bat,

I pråfentirtellerfôrmig, ber Edflund mit Drufien be= reşt: " s"

2 trichterfơrntig; Der Sd)lund naclt , : : :

b6 Die Љlumen figen wirtelfor:

Siannsharni(i), Androface.

Edilubelolumen Primula. mig an langen Stengeli, fino pro̊ientirtellerförmig; bieStanb. fäben an ber blumenrơbre, bie Rarbe fugelformig

- Malierviole, Hot-

cc Der \$̧lúthenftand allberş. tonia.

B. Die Blume rabfórmig

I Die Riaplel kugelförntig

2. zugerpiset, Iofit)aalig

3. Ifchaalig, zerfpringt mach ber Dues Evfintadie, Lyfimachia.

re it: 2 f̧albutugeln; bie Staubgefá̉e fonumen aแts einem båntigen Ringe,

ber ben fructitnoten ungiebt "Saudbeil, Ana-

2 Die Rapfel follieft cine beere ein; bie gallis. Slumenro̊bre febr furz; ber Sahlunb bervorragens

- Sifiveinstrod,Cy-

3 Die Rapfel entund, mit bem Reldje clamen. umgeben. 
2. Die Bblume an ber innern flåche Jottig; bie నarbe 2fpaltig. = Sottenblume, Me.

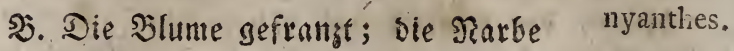
2lappig $="=$ " TSaloidmidie, [2. Die Slume glocfenformig, zerriffen, Waldfomidia. sielipaltig; die Japlel vieljăfnig,

b eficherig; - Colbanefle, Sol.

an Die Blume ift tridyterforrmig,

I in galten gelegt, in ben $\mathfrak{x}$ sin=

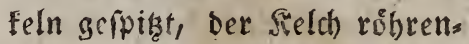
formig, ectig; bie Suapiel 4 fdraas lig $=$ (1)

2 etwas ungleidy, ftumpf; bie Stedapfel, DaStaulgefäfe fino umgebogen; Die Siapfel hat einen Decel Silfentraut, $\mathrm{Hy}$ -

66 Die Shlume iff rabfirmig, etwas ofcyamus. ungleid); die Staubfäben zottig; Die Sapfel vielfaamig 3 = SEollflume, Verec Die şlume ift glodenformig, bafcum. faltig, mit 2 Rarben; sie Siaprel bat in jedem Fache zmeen Saas men

c 3fächerig, 3fhaalig, vieffamts; bie 2Binde, ConvolBlume 5theilig; ber biocen if nit Den unten flach georúcten Trågeru verichlofiem, bie ?arbe ift zwey= ppaltig

bb in einer 2fácherigen beete; vulus.

a Die Şlume rabformig

I Die Etaubbeutel fino faft sufaumgemachien, an ber Epik̨e 2lócherig , Naditidattent,So. lanuin. 
2 Die Stantgefâke gegenein. ander gentigt; die Secte ift in ben aufgeblafenen Frudit: feldh eitsgerdiloffen " SubenEirfate,Phy.

5 Die Slume glockentarmig, bie falis. Staubbefibe ausemander ftes bend $==$ 2Bolfstitfite,

B. über Dem frudttnoten; Die frucht iff cine Siapfel.

I Die Shltme glodfenformig; Der boden von bem, unten flacige: brudten Theile ser Staubgefáae bedectt; Die Siarbe 3ipaltig, die Stapjel ofnet fich an ben Eeiten Blocenturumeg

2 Die Silume prafentirtellerfurmig; Atropa.

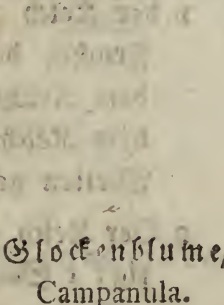
Die Ctaubfíben bededt ron Den Echuppen ser Slume, bie Staprel sficherig = $=$ = "Ylunge, Samolus.

3 Pie Şlume radformig, 5theilig;

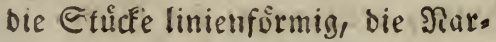
be 2-3fpaltig, bie Siapiel 2-3fas: cherig. = = , siapunjel, Phy-

II. Die Slume 5 blåtterig teuma.

a unter bem fructifnoten

a untegelınẩig, mit einem Epcrne;

1 Die 5 Seldhblätter lanjetfirmigh unten funpf, kiber Dem Brunbe angemactien : : Deilden, Vicla.

2 Der Relch $2 b$ läterig : = " Epringfratit, Irs-

b tellerfo̊rmig; bie B̉lumentlåtter uns patiens. ten sufaumgeleimt, zno tellerförmige Narben, eine uber Der andern

Cinngtůn, Vinca, b siber 
b นี้ (d)aftlide relch ro-20blátterig, unter Dem Srudthtenoten; Der fonberbeitlidje über semfelben, 5 fpaltig; sie $\mathfrak{B l u m e n b l . ~}$ linienformig; Die গ?arbe ber ?anoblưtg: d)en 2fpaltig; ber mittlere ungertbeilt, feilfôtrmig, fottig $=$ - Jafione, Iafione.

III. Sie Şlumen feblen gang,

I ber Sieldh Iblatterig, rubrig, ůber ber Frudt); Die Staubgefåze fommen aus Dem Sield)e; ber einjelne Saame mit Dem Relabe befleibet, oben mit beffen Sheilen gefroinet " " Leinblatt, The-

2 Der Reldh 5 blätterig; Der Griffel 2ppal: tig; I Saame linfenfórmig fium.

פieloe, Atriplex.

\section{Mit 2 Staubwegen.}

1. Die Slutthen einjeln, Dber in 2leften.

A. Die slume Iblatterig; die Siapfel Ifå= doerig, 2fdaalig, vieliaamig; ber Eirif.

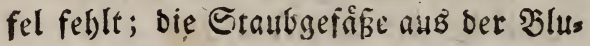
me, welche

a rabformig ift ; an Girunbe jebes Slumenftúcfes fino zwo 2̧erties fungen mit Franjen umgeben, ber

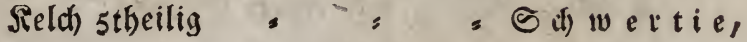

b roffrig, unb fo wie ber Seld, Swertia. 4-7ipaltig ift; bie গ?arbe ein: facb; bie Caamenbóben nach ber

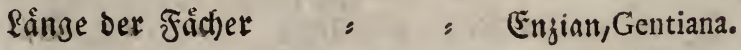

B. Die Slume feblt;

a Der Reld) 5blåtterig, mit I nacften Saamen, 
a Det nietenformig, und in bem etras fleirchigen Selchboden ein: geichloffen ift

Pinnen, Beta.

b Der linfenformitg uiber bem ecfigen stelche iff,

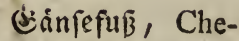

b Der field) 5theilig; eine vielfanmige nopodium.

Siaprel; 8 oder ro Etantbgefä̉ie, wo= von die Şalfte beutellos ift.

3irufftraut, Herniaria.

II. Die sblutthen gebåuft, ftiellos, auf einem fegelfơrmigen Sluttbévoden aufitizeno, mit. telB Spreuer von einanber abgeronbert; ber gemeinfdhaftlicte $\mathrm{llm}_{\mathrm{m}}$ h)lag mehrblåt= terig, lang; ber Sield) länger, als fein Silithchen.

III. Die Sluthen in Dolden, jebeg \$lưth=

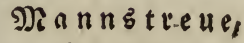
Eryngium. d)en 2faamig; bie Gaamen nacft.

A. Dolden unb Doldchen mit f̧ưllen (mit gemeinid)aftlidsem und befondes. ren Umidhlage)

a alle Sklưmchen fruchtbar,

t bie sußBern geftralt, bie Sctutte ungertbeilt, lang, lanjetfórmig; bie Saamen faft tellerfórmig, am Ranbe geferbt = " Diebltaut, Tort ungefiralt. dylium.

a Die Slumenblåtter bersfor: mig,

I eingebogen ausggerandet, bie Frucht långlidht enfơrs mig, geftreift, bie Ulm= fhlagblătter linienförmig sogeineft, A thamantha.

2 Die 
2 Die Frtudt fapt fugelformigi 5 freifig benderfeits geferbt; bie Sallldhen eilleitig, mei= ftens nut abläterig = Edierling, Co.

3 Die fructit faft enformig, nium. geftreift

a etwas linglich) gufamm: georufft ; bie Sctúlle $a b=$ warts gefefret "Silze, Selinum.

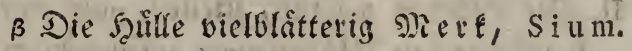

b Die Sjlumentlátter eingeroll, gants.

I Die Futht bevserfeits mit 5 Furd)en; Die Sjlumehen gleid); sie Şuillen mit

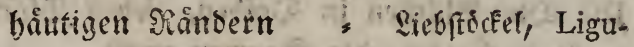
2 Die frucht isublicte, jufant. fticun.

8) gebruidft, ageftreift; bie * Şulldidir lânger als die Dolodjen, 5 blitterig = Dutdwwadi, Bu. c Die Slumenblätter etwns pleurum. flach

I Dod) eintoarts gefruintmt; bie Frucht rundicht, ecfig, bicist, bevoerietts mit 3 Furchen " " "Engerwury,Anger

2 Die Frudht enformig, ges " lica.

- ftreift, bie Dolde ganz arm nIt Şlümchen, bie. F̧ứlle umgefăbr vierblätterig = Sifon, Sifon.

b Die )Rittlern unfrudtbar

a Die Dolven falt fopfformig, sie Fruidte feifborftig : Sanifer, Sanicula. 


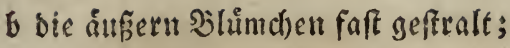
Die Srutldhen gefiedert = Sselbribe, Dau-

c Die ätBern Sllumb)en geftralt; bie cus.

Slumenbl. eingebogen, auggeran: Det, bie Frucht Deutlich ffeiffor. frig; bie Şưllen unjertbeilt " RTettenesubel,

D Die Fund enfirmig, benderfeits Caucalis.

geflügelt; (Die f̧ưfllen febr furş) f̧an:ftrang, Peu.

e bie Shlumenbl. éingebogen, auzs. cedanum. gerandet, weglebeno; die Frudt långlict)t, mit 8båntigen Slůgeln (bie f̧üllen febr furg)

f bie Slutthdien ber Mitte flein, Eaferfinut, Laferpitium. mit gleid)grofien Shlumenblåttern, bie bes llmfreifes grofi, mit un= gleiden Blumenblattern; bie Fricht mit bem Seldhe getront Oenanthe, Febert

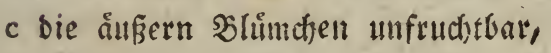
soloe.

bie fonderbeitlichen Şưflen lanzet. formig, wegftelsend, gleidlang, länger als bie Ctralen, geförbt 2fftrang, Aftrand bin uns wieder unfruchtbate \$llim= d)en, bie Frucht elliptifd), flad): gebricft, ausgerambet, in ber 刃itte geftreift; die fautlen abfalleno =

B. Meiftens eine allgemeine Iblatterige cleum. Sctille, bie befondere fehlt; Die blus menblätter eingebogen, auggeranbet, am Siưcfen mit einem Sitele; bie frucht långlidt)yformig s Silmel, Carum.

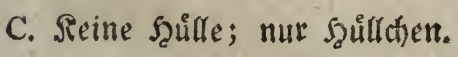
t Alle \$llumchen find fruchtbar 
x bie mittlern Slündhen finb flei. ner, bie Fruttht glatt, mit bem Sieldue gefrónt

- Drexdefanmen,

2 bie Şlumenbläter ziemlich flach, bie Frudht gefurcht Phellandrium.

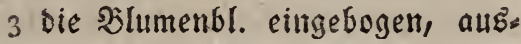
geranbet; bie Frucht rundicht, zufammgeoruift, in ber Nitte bo: derig, mit einem sande umge: ben, (NB.) zumeilen bat fie Şülle uno fretllden

SBRuterich, Cicuta.

4 bie ફ̧น̊lllhen vielblåtterig; bie Mieifterwurz, InSlumchen etwas umregelmåfig; Die Saamen evfurmig geftreift; bie Dolbe Eugelfôrnig mit I ober 2 Umidhlagblattchen peratoria.

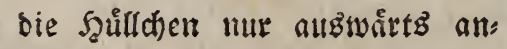
gefeţt, 3-5blåtterig, die frudt geftreift = " Bileine, Aethura.

t Die mittlern Slümd)en unfututbar

a bie Frudlt lang, glatt, bie $\mathfrak{b l u s}$ menblåtter eingebogen, ber ffơr

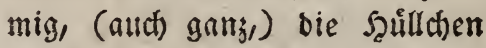
abwairts gerichtet, vertieft = SåtbetEvof,

b bie Slumenblåtter ungleich, aนş, Chaerophyllum. gerandet; Die Frudt)t långlicht, pfriemenformig $==$. Sistbel/, Scandix.

D Dbne alle Şưllte.

a bie mittlern Sluimchen meiftens un. frud)tbar; bie Frud)t elliptifd, flach sufammengebruckt; bie Dlumenbl. eingerollt, gans

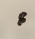

b bie Şlumd)en alle frudftbar, bie Frucht geftreift, evferemig
Selel, Sefeli. 
I långlid)t, bód blåtter herzfofrmig :-

Beişuß乃, Aega.

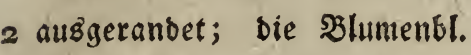
glattrandig podium.

Fendiel, Ane-

3 fehr flein; bie Slumenbl. ein: thum. gebogen " " Eilge, Apium.

4 bie Rarben faft flugelforrmig; bie Dolben vor bem Sllutbe. ftand ůberbángend

= Bibernelfe, Pimpinella.

\section{Mit 3 Stanbwegen.}

Seld) uns Silume sblätterig, regel,

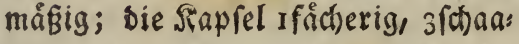
lig

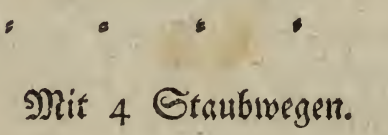

Mit 4 Gtaubwegen.

Det Reld) ift 5theilig; Die Sllume 5 blåtterig; Den Frudtffnoten unt: seben 5 Drůien, Drvon jede mit mebrern geftielten fugelid)ten Drǔs. chen belergt ift

Şน์โnerdarm, Alfine. 
Mit viden Stantwegen.

Der Selit) 5 blätterib, tiber Dem Girums se angerwadjen; bie blume bat 5 pfrientenfơrmige Slåtter; bie Sans uten fins unbedeclit, uns am ralgen: formig auşwach fenden Frud)tboden

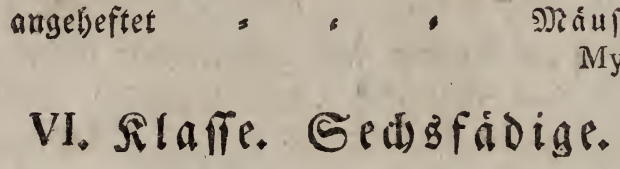

\section{D)it I Gtaubwege.}

Şalbgrâjet.

Der Reldh ift 66latterig; bie \$lume feblt.

a. Atis bcm Sufen eines Şlattes fơmmt cinse tualgenformige retule, Didbt mit Shlutben bedectit $=\quad=\quad$ S SnImub, Acorus.

b bie Shitben in Enaulformigen, gebณ̊uf ten Aebren, ober in Sifipen, auch ein. geln, bie Sipapfel if Ifächerig : Sinfe, Iuncus.

\section{Sĩñ åt.}

I. Nit einer Shathenicheide; Die Slume Gblåtterig.

a น̊ber ber Frudbt

I bie 3 innern shlumenblątter mechs feln mit ben åußern ab, fino feirs zer, und oben berzformig ausge= f(d)utten; bie Shluthenicheibe in ber Dittte, unb an ben Eeiten baiutig, alf Dein Rlucten bobltielig, an ber Spise auggeidnitten • Sonneeglode, Galanthus.

2 bie 
2 bie \$lumentsl. gleich, an betr Spis ken bicklid)t; sie blume gloden. formin

3 bie grof̧e Slume entbålt nody eine Fruflingsglocte, Leucoium.

innere rblåtterige an ber Niuns sung atigebreitete gefarbte SJlus ne, welche finme ein soniggefä neun ; Die Stanbgefófe find in

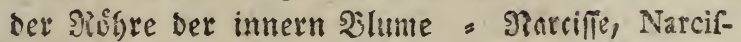

b unter ber Frucht; die Şlument. fus. lanjetformig, bie Siapfel orevpeltig, 3 fîcherig, bismeilen 2 Wlütbenfchei. 1 Den
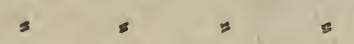

Sast (1), Allium.

H. Weder Blutthenidheibe, noch Reldh.

A. Die Slume Iblâtterig,

a 6fraltig; bie beere gefledt, 3 laamig, 3 fücherig

b Gtheilig

गanblume, Convallaria.

a oben an Fruthifnoten fint 3 Şnits: d)en; (bie 23 urgel eine 3miebel) Şyazinth), Hyab die Silumenroibre fur', walsenfot: min, bie Staubgefốfie abwärts gebogen; (bie 2rsurgel frotig) Epedelitie, He-

c sie unten breitgebrudten Gtaubs trioger becfen ben Frudiffnoten affooille, Afpho-

c uแregelmåfig, rọbrig, ůber bem cinthus. merocallis. delus. Fruchtenoten am Grunde bauthig, Det Rand endet fich unten in eine lange zungenforninge Platte; Die शarbe 6theilig; Die Rapfel 6fächerig. - Dfterluzen, Ari-

B. Die Shume 6blitterig ftolochia.

2 bie Slåtter wegftehent 
a bie Staubgefâfie wollig - Bauntume, An-

b bie Staubgefåfe alle fabenformig,

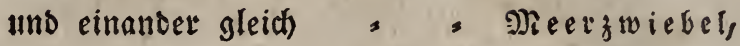

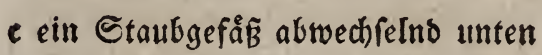
breiter, als das andere = Sogetmild, Orni-

b bie Slåtter gerabe auffetene thogalum.

a rebr lang; bie Staubgefåze refre furg; Dér Oiriffel 3fpaltig; Die

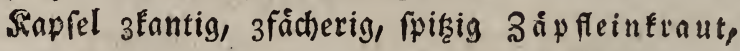

b bie 3 innern ஒlåtter mit ben åu. Uvularia. fern abwectielno, an ber Epise umgebogen, bie beere 3fädherig, in jebem Fache 2 Saamen = Epargel, Afpara: c glecfenfơrmig gus.

a faftig, fpróbe, bie Rapfel 6furchig, 3 fächerig, 3 f haalig; bie Edhaalen burch gegitterte Şaare verbunden;

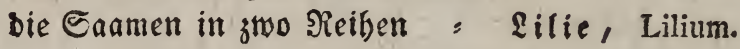

b ber frudthtnoten ift eine 3 fantige Såule obne Griffel : ' Tulpe, Tulipa.

III. Der Seldh Iblatterig, zmolffipaltig, glos denfurnig; bie \$llume fellt oft, fonft ift fie 6blâtteris, fờm aus bent Sieldhe; bie Raprel 2fåcherig s " " Pepliz, Peplis,

IV. Der Reld 4 blåtterig; Die Slume Erews: fơrmig, 4 blätterig

A. Die Frucht ein Sdhottchen

2 bas Sd)sttchen unausgerandet

a etwas zulammengebruict, am Ente griffellog, bie Edjaalen ziemlich flad, mit ber Stheibes wano parallel

Eeinootter, Myagrum. 
b faft fugelfórmig mit einem fegel. fơrnigen Giriffel an Ende; bie Sdjaalen loffêlfờmig : Scungerblämdien,

c elfiptifh), sufammengesruedt, volt: Draba. fommen flad); Die Sd)eidemans farallel, bie Sielchblätter am Grumbe baudsig

- Mionoviole,Luna-

b Das ๔afittden nusgerandet, ria.

I ber;formig; bie Echanlen fiels formig, wiberfinnig s Siefie,Lepidium.

2 verkebrt berjfơrmig; bie Ectjaa. len mit Fielfornigem Sande Toifdeleraut,

3 aufgetrieben raud); bie E(haa= len bơderfơrmig, fumpf = Isffelfraut, Coch-

4 faft fugelforrmig; bie Edjeibes learia. wano parallel; einige Troiger baben cinen einwairtz ftebenden 3nbn $=$ Z(t)ife, Alyfium.

5 jufanmegebruckt, flad), boppelt, (iil Der Mitte von Der wider. fiumigen Sdbeibewant zufanımen gezogen;) bie Cchaalen faft tellerformig; Die Reldblatter am Crunde bauchis

Doppelidils,

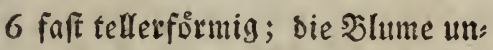
Bifcutella.

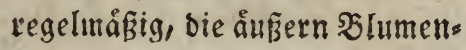
blâtter gre̊ßer $=$

Salternienf, Iberis.

B. Die Frudt eine Cdhotte

Thlafpi.

a faft walsenfórmia

a furingt elaftifus mit zuruictiges rollten Edbaalen auf; bie ミar: be ausgerandet - 3nfnfraut, Dentaria. 
B pringt mit geraben Schalen auf ; Sieldh und Sllume find lofe, offen, faft regftebeno : Brunnenteffe,

c zwifhen jebem furzern Trăger Sifymbrium. uno bem fruchtfnoten if eine Druife, und eine andere zmis fchen jebem lângern Tringer, unb beim Sieldie

I bie Camen fugliḑt, Sogl, Brafficas

2 bie Edjotte von ben Sna: men aufgetrieben

t $\mathfrak{u}$ ber benfelben borns artig, verlångert, ets was ablang, rauch Genf, Sinapis.

\pm vielhócferig, ober ges gliebert

Rettig, Rapha,

b gufammges rickt, nus.

a maljenruno, linienfortmig, fpringt mit juruectgerollten 巨chaalen auf; Die Rarbe zertbeilt " Carbamine, Car-

D Der Seld) gefdiloffen mit 2 am damine. Grunde boofferigen Slâttchen, auf benden Geiten bes frucht: Enotens cine zalinfe̊rmige Drůie, bie fid) bey einigen verliert, bey andern wridffi ; bie Saas men flach $=$ = $=$ Levéoje, Cheiran-

- Păulenfórmig, genau vierfeitig; ber thus. Reld) ziemlid) gefhloffen; bie fürs zern Traiger baben Druifen am Erunde Şeserid), Eryfi-

d linienformig, lang; bie Narbe ein. mum. fach; vier Drůfen am Shlutheboden,

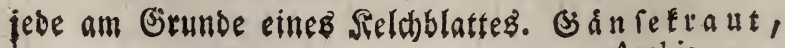


e vierfeittg, fefre lang; Reld) uns silus

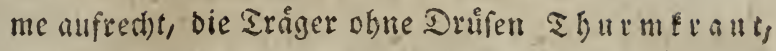

- f fur\}, långlicht, langetformig, Ifâtbes Turritis. rig, rfaamin, 2fchaalig; bie Echaa= len fohifformig " =" $\mathfrak{W}$ aib, Ifatis,

g geftecift, jufanmmedrucft, flach, etwas gegliedert; an jebem furzern Sråger ift eine Drůfe, bie feinen Grund un: giebt; zwell entgegengeferte Reld)s

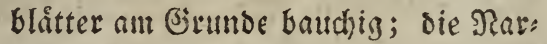
be untelt gablicht, die Stricke sben

einanser anliegend
Mit 3 Gtanbuegen.

A. Der Selctif felglt,

Sataitutiole, Het fperis.

I bie Slume Iblatterig; bie Ro̊tre feft lang, Der Siand glocienformig, ótheilig,

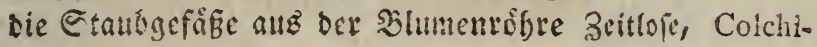

2 bie Slume 6olätterig; 3 vielfannige Siaplelt

$=3=$

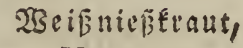

3. Sieldh und Şlume 3 blátteriy,

Veratrum.

a I Enamen, nacft, brenfantig, won ser (idi) (a)licẽenoen Sblume bedecft; bie Narben zertl)eilt " " , 2(mpfer, Rumex.

b $3-6$ federformige Narben; sie Siapfel ftumpf, 3-6föd)erig, Die Griffel feblen Drenfpi Trig-

C. Der Reld) 6łlatterig; Die Blume febit lochin.

a 3 Giriffel, glatt; bie Rapfel sfeitig, 3 fůcherig; bie ミ̉lumenblåtter linienfór. mig, frumpf

Sceriterie, Herite-

b bie Griffe! feblen, 3 aufgeblafene, riaas ria. mige Siapfeln

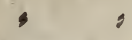

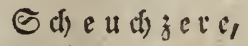
Scheuchzeria. 
Mit vielen Gtaubweget.

Relch unb Slunte. 3 blátterig; mebrere Saa: men

\section{- Rlafie. Siebenfádige.}

I Der Sieldi) 7 blátterig; Die Slume 7tbeilig, flach), regelmåbig; bie Seere faftlos = " Editmitaut,

2 Der fielá) Iblåtterig, fünfzånig, Trientalis.

baudhig; bie blume fúnfblåtterig aus bem Seldie, bie Sapfel bat 2 beutlidie, und I unbeutlidjes Schaa:

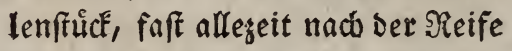
nut Ifä̀cherig, Ifaanitg, vor Der Neife 2faamig = = Tob́aftanie, Aefculus:

\section{Rlaffe. 2(dtffádige.}

\section{Stråude, und fitadartige Siáter.}

I. Bollkommene Bhitben

A. unter bem Fruchtfnoten; ber Selch 4 blâtterig; bie Slume 4ipaltig; bie Staubgef. aus bem \$lútbeboden; bie Saprel 4 fäctherig, , "J̧eide, Erica.

B. über Dem Fruthtenten;

a Der Reldh 4fpaltig; bie Slume 4 blät terig; Die Sapfel malsenforrmig; Der Saame nactet Rúbrapungel,

b ber Reld 4åannig; bie Sblume balt. Oenothera. d)ig, glodenfơrmig, bie ఇưnbung 4รăhnig; bie Staubgefá $\mathfrak{B e}$ aus bem Slưtheboden; bie Seere 4fåd)erig, sieliaamig : Sceibelbeete, Vaccinium.

II. UH: 
II. Unvolffommene Şlutthen

a ber Reld feblt ganj; bie Slume rôh= rig, vieripaltig; I Saamen, geidnabelt Etellere, Stellera.

b Der Reld) genau mit ber Stume gufam. mengewadhren, viertbeilig, gefâtbt; bie Seere fleifhig, Ifaamig = Daphne, Daphne.

\section{Ir\&uter.}

I. Mit I Stanbwege

Der Relch 4fpaltig; Die Slume 4bläts terig; Die Sinpel lang gezogen, (eine Sdotte) unter ber Slüthe; bie San; men mit einer feacrfrene

23eiberid, Epilo. bilim.

II. Mit 2 Staubwegen

2 ber Seldi) 4-5fpaltig, gefåtbt; bie Slus me fehlt; bie fiapiel 2 gablig, Ifád)erig, vielfaamig

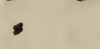

b Reld) uno Slunte 4blatterig, bie Raprel If nicherig, 4fochanlig

III. Nit 3 Staubmegen; ber Sield) 5 theilig gefôrbt; Die \$ুlume fehlt; I lnแjetfôrmi. ger oder 3 fantiger Sname s

IV. Mit 4 Staufwegen

a Reld) und Slume 4 blåtterig, bie Slumen: blätter fohnal; bie \$eere ffädherig

Einbeete, Paris.

b bet Relch 2fpaltig, unten; die গুlume 4-5fpaltig, oben; bie 5̧ecre 4-5fäd)es rig, mit bem Reldye sufammgewad)ren Mit I Staubrwege; ber Relch 5paltig; Die פllume 5 blătterig
Siltzitiaut, Chryforplenium.
9) Moehringia.

Wegtritt, Polygonum.

Si Tamétaut, Adoxa.

2horn, Acer.

IX. 2inalle. 
44

\section{Silafie. Neumfádige.}

Nit 6 Etautwegen; ber Selch fehlt, bie Slume 6blåterig, 6 vielfanmige Rapfelm 'Baflerviole, Butomus.

\section{Riaffe. Zefnnfidige.}

Stråucber, und frraudbartige Sirůuter.

A. Mit I Ctaltbwege, ber Reld 5 fmaltig, ober 5theilig; bie Slume Iblätterig, 5fpal tig

a enfórmig; die Staubgefõfe aแB bet Sblume;

a mit einter Sapfel $=\quad=$ 2ndromeda, Aile

$b$ mit eitter Seere; sie Slume am Girunbe Durchfichtig , SBâtentraube, Ar-

b flach; Die Siapfel foringt am Grunbe butus. auf

c faft tridhterfo̊rmig; Die Staubgefo̊ne aus Den \$lútheboben, abwairts ge= bogen; (mit einer siapiel) " Jlpenbalfam,

B. Mit 3 Stantwegen; bie \$̇lume 5 blatterig

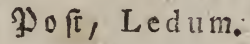

a ber Reld) I blätterig, bauchig; bie \$lus mentåtter mit গågeln, ůber bentels ben eine Eleine getbeilte \$latte ant Grumbe ber silumenblattplatte; bie Sappel 3 fächerig . "Silene, Silene.

b ber Relch 5 Glátterig, bie Slumenbläts ter febr Hlein, ausgerandet; 5 Tråget wechfelmeife beutellos, die Rapfel Ifåd)erig, 3idjaalig, 3famis . Egrterie, Cherleria. 
กำuter.

I. Mit I Staubwege

a bie Sblume feglt; ber Sield) 8-Ioblat, terig, Die 4 oder 5 ånßern auswairts bauchig: Etaubgefäize 8-10

Mionotropa, Mo notropa.

b bie Shlume 5 blåtterig;

a Der Relah 5 blätterig; Die Blumen. blitter ffehen meg; bie Staubtråger fino mit punftformigen Drifen bes fäet; bie 5 Siapfeln verbunden = Diptam, Dictam*

$b$ Der Reld) 5theilig; die Rapfeln $5 f$ a: nus. cherig, an ben Sianten aufipringent; bie Staubgefä́pe aus dem sluttbes boben

H. Mit 2 Ctaubrogen, ber Reld) Iblätteris; ISBinterguin, $\mathrm{Py}$ rola.

a bie Slume feblt; bie Staubgefåne aus Dem Sxelche; bie 2 Saamen nactit, vom Shalfe bes Reld)es eingeichlofien = Rnauel, Sclerans

$\mathrm{b}$ bie $\mathfrak{3 l u m e ~} 5$ blätterig thus.

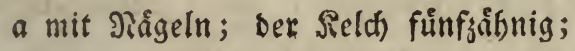

I walgenfornig, am Grunde mit 4 Schuppen bejetit; bie Rapfel mals zenfơrunig " " Nelé, Dianthus.

2 rofbrig, am Grunbe fahuppentos; Die Siapfel langlicht . Ceifenfrant, Sa-

6 nasellos; bie Siapfel Ifådjerig; ber ponaria. Relch 5 theilig

I ecfig, glodenforrmig, an ben Ecfert gefôrbt; Die Siapfel Eugelformig Brypstraut, Gyprophyla. 
2 Euts, fpişig, die Rapiel spdnab. lig

III. Nit 3 Staubrwegen;

Eteillbredje, Saxifraga.

a ber Seld I Ilatterig, aufgetrieben; bie

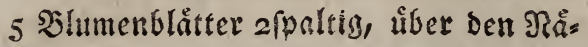
geln nackt; bie Riapfel 3 fâdberig . Ekliesweid, Cucu-

b ber sieldh 5blatterig, wegftebeno; bie balus. Siapfel I fädherig, vielfaamig

a bie Slumenblatter atheilig - Mecirich,Stellaria. b_____ gang : Sandétaut, Arenaria.

IV. Mit 5 Staubrwegen; Die Slume 5 blåts terig.

A. Der Reldh Iblåtterig,

2. 5fpaltig; ant ล̊ußern Grunbe jebes Fruó)tunotens eine fleine હa)uppe; 5 Fruchttnoten , ,

b 5 ร̧̊̊็) geln;

I bie \$latten ftumpf, ungertbeilt; bie Rapfel Ifädherig - Tiaben,Agroftem-

2 Die \$latten einge(d)nitten; bie Siaprel 5 fåcherig,

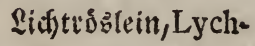

B. Der Reld) $5^{\text {blatterig }}$ nis.

a bie Sllumenblátter an ben Någelu verbunden; : I Raprel, stantig, an Den Stanten aufipringend = Saurtlee, Oxa-

b bie Slumenblatter 2fpaltig; bie Rap: lis. fel iffnet fid) oben =" = Şornktaut, Cera-

c bie Slumenblatter sans; die Sapfel enfoirmig, 5fdaalig ftium.

Sparté, Spergula.

XI. 2ilafie. 


\section{Silaffe. 23ielfädige. \\ $\mathfrak{B} \mathfrak{a} \mathfrak{u} \mathfrak{m} \in \mathfrak{u} \mathfrak{n} \mathcal{D} \subseteq \mathfrak{S} \mathfrak{x} \mathfrak{a} \mathfrak{d} \mathfrak{e}$.}

I. Mit I Ctaubrwege

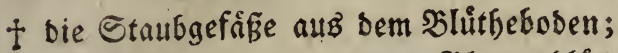

Der Reldi) 5theilig; bie 5 Şlumenblát=

ter am Ende geferbt; Die Riapfel tro:

cfelt, fugelfôrmig, 5 fîcherig, es wirb

aber nur I Saamen reif, bafjer bie

Siapfel meiftens nur ffähtherig fheint \&inse, Tilia.

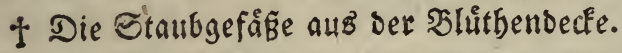

A. Der Reldi itber ber Frutht, 4-5thei,

lig; bie Sllume 4-5blåtterig; bie stapfel 4-5fä́heri̧, vielfaamig : Rronenjafmin,

B. Der Seld unter ber Fructst, 5ipals Philadelphus.

tig; Die \$llume 5 blåtterig

a ber Stein ber Steinfrud)t giem.

(ich) flach) georuicft, ober erbien= foremig, glatt, allemal mit vors

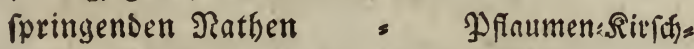

b Der Stein ber Steinfrucht runz:

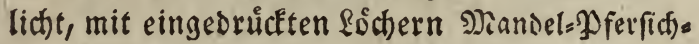

II. Nit 2 Staubwegen, feltner mit I; ber baum, AmygdaReld) oben, 5ipaltig, bie \$lume sblåtterig, bie Seere 2faamig " = " Sagedorn, Cra-

III. Nit 3 Staubwegen; Der Relch oben taegus.

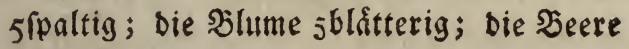
3raamig

IV. Mit 5 Staubwegen; Der Relch oben,

Dogelbeerbaum, Sorbus.

bie SIlume 5 blåtterig

a mit ainer 5 faamigen Seere - Dripel,Mefpilus.

b mit einer 5 fäh)erigen, vielfaamigen Rernfrud)t

Bitn:2fpfelenum,

Pyrus.

V. Mit 
V. Mit vielen Staubwegen; sie Shlume 5 blitterig

a set feld) oben, sipaltig, Erugformig; sie'beere fleifthig, mit ben fieifen Sieldhifheilen gefroont; die Snamen zolstreid, freifoorfiti, an ber Echaale ber Fruicht angewadjen $=$ Siofe 1. Rofa:

i ber Steld) unten, 5theilig; sie Seere ans einzelnen Kleinen, einfaamigen Beerdfen Jufammgereşt

Erombere, Şim: beere, Rubus.

Sigentliche uno ftraudjartige firauter.

1. Die Staubgefốne aus ser Şlưthendedłe, mit 5 Staubrogen.

a ber sield) 5ipaltig, sie ßlume 5 blåts terig; 5 audh melgrere vielfanmige Siapieln = " = " Epieraie, Spiraea;

$\mathrm{b}$ ber Sield 5 - Iorpaltig;

a bie \$lume 4-5blatterig; bie Reldf) fthdie mechlelmeife (A)måler,

I ber Gamenboden wirb bees renformig, und fâllt ab = Evtbeete, Fragav

2 Der Saamen nadit, an ben ria.

fleifdlofen Saamentoden bes feftiget $=$. - Fotentilfe, Poten-

3 bie Saamen jahlreidh, mit tilla. geglieberter Branne = Marymizigeum.

6 die Blume 5 blitterig, die Sifumen= blätter fleiner als̄ ber feeld), lan: aetformig, fpiz̨ig; Der Frud)tboden enforemig, ichmammig, b!eibent, nactit

Siebenfinge!s Eitat, Commarum. 
c Die Shtume 5-8blåtterig; bie Grifo fel aus der Seite des Jrudthno. tens; bie Eaamen mit baarigen Sd)wåmen

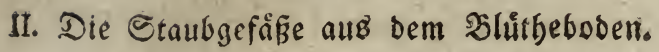
$\operatorname{SBa} \log \delta$ ttiti Dryas.

\section{A. Nit I Staubrome;}

2. Der Sield) I blåtterig

a 2fipaltig, bie Bhtume 5 blåtterig, bie

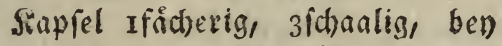
einigen 2 inten sifnet fie fid nach Der Quere

Wortúlack̂, Portu-

b 3-4ifaltig, altf ben Frudthtnoten laca, aurfithento bie bllune fehlt; bie Siapfel leberartig, gefrónt - Seafelmurg, Afa-

c I2paltig, bie \$̇lume 6bläterig aus rum. Dem Fielche; die Sapfel afähtherig, vielfamig

25. Der Reld) 2blatterig; bie Blume

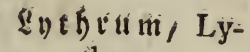
4 biåtterig.

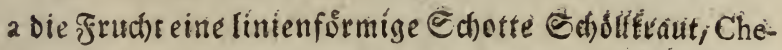

b bie Siapfel Ifócherig, mit bek breh lidonium. ten నarbe gebecit, und anter bie: (er burd)! lochert $\quad+\quad$ Mrogn; Papaver.

(3. Der Sield) 4-56låtterig

a bie blume hat 4 ftumpfe $\mathfrak{b l a ́ t t e : ; ~}$

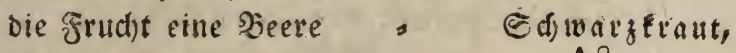

b bie blume 5 blatterig; (sie Frudt) Actaea. eine Stapfel) \& Sielchb(âttchen Heis ner $="$ Ciffentsadjen, Ci-

c bie $\mathfrak{B}$ hume vielblätterig, in 2 oder ftus: mebrern encentrifthen Reiben; bie Siapfel fieifhig, wielfficherigy abgefiuget

Seevoie, Nymphaea.

B. Mit 
B. Nit 2 Staulbwegen; die Slume 5 blät=

\section{terig}

a Der Sield 5 5̧ånnig mit einem Sleben

fetde; 2 Eaamen im Neldgrunbe Doermennigh

b ber Sield) 5 Ulätterig; die (Jiriffel feb. Agrimonia.

len; 2 vielfanmige fiapfelm - Dfingftrole, Paen

C. Mit 3 Stallbwegen onia.

a ber sield) Iblátterig,

a jertfjeilt; bic şlumenbläter jels ichliffen, bie seapfel oben offen, Ifídjer:ig

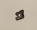
:

$\mathfrak{B} a$ แ , Refeda.

b die Slume 4-5blâtterig; die Rap. fe! zlaamig

b Der seldh 5blåtterig, gefårbt,

a mit einem Eporne; bie Slume Iblatterig, gefpornt, ber Sporn in Den Reld́biporn verientet

6 mit ber Slume fufaummgeleimt; Sitteriporn, DelDns oberfte Relchblatt belmfo̊rmig; zwev) voll Dell Etaubgefåzicn ges

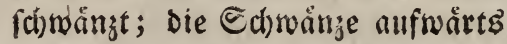
gefefrt, am Ende gerollt = Sturmbut, Aco-

D. Nit 5 Stallbmegen

a Der seldy fehlt; mehrere slumen. blatter, unb in verfatiedenen concen:

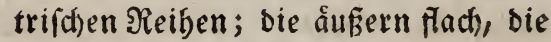

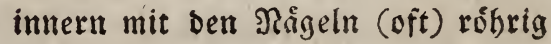
aneinander gemad)fen; Die platten

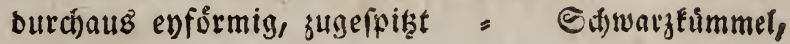

b Der Setch 5blatterig, gefărbt; die Tsolfsmild), Euphorbia. phinium. nitum.

Slätter flach; bie 5 গ̧lunenblåtter mit bem Seldhe gleichfärbig, mechiel. weife, an ber Seite angewachlen; jebes trichterfórmig, 2lippig : 2lglen), Aquilegia. 
E. Mit mehrern Staubregen;

a init einer Slluthenid)eibe; bie Frudtsts fnoten machien in mebrfitamige bees ren aแs; ; inner ber Ect)eide ein läng: lichter fingerformiger Bapfen

a auf melda)en bie Staubgefáfe mit ben vielen Frudjtfnoten blumenlos vermengt find = Calla, Calla.

6 weldher oben nackt, in ber Mitte mit vielen Etaubgefåfen, ties fer unten mit Etempeln beferet ift; bie Echeide fapusenformig 2tron, Arum.

b Saeber Sieldf, nod) Sluthenidheibe.

a Slumenblitter $4-6$, in einer cinfachen ?leite

I mit vielen feberformig ge: (d)wänten Saamen - SBaldrebe, Cle-

2 mit vielen ungefhrwånten matis. Saamen, Die Slumenblät: ter rebr binfällig : Biefentaute,

3 mit mebrern, If åd)erigen, Thalietrum. an ber obern ?ath aufs fpringenten, vielfantigen Rapieln = = Dotterblume,

b Slumenblåtter แmgefåbr I4; Caltha. mefrere, faft rumbftielige, balb. blümdenformige \$latten vor Den Etaubgefǟenen - Engelblume,Trolc viele જ̉lumenblätter in megrern lius. $(2-3)$ Reifen; Die Saamen nackt, zablieid)

2fnemone, Anemone. 
c ber Reld 3blätterig; viele blumen,

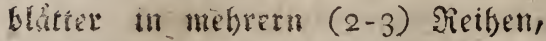
Die Eanmen nacit, zoblreid) = Eeberfraut, Hepa-

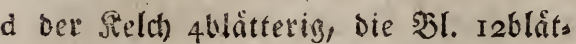
tica.

terig; viele nacte, geidmantmte Ena: men

$=$ :

Doppelolume,

e ber feld) 5 , aldi) mebrblatterig Atragene.

a groß́, gefärbt; Shlumenblätter 5, aud) mebrere, fletn rofbrig; bie Griffel reitwints in Den Fruds) Enoten eingelafien $=$ Niefsurty, Helle.

6 Shlmenblitter $5-15$ in einfadjer borus.

Sieibe, cinfache viele Enamen auf einem fitumf fegelformig werben: Den Samenboden

c Shlumenblătter 5 ; eitre Drúfe in Fellet dod en, einem Gruldsen eines jeden গa gelz, oft mit eimer Echuppe bes

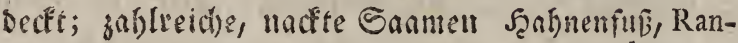

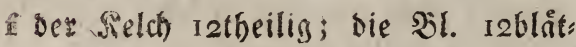
Adonis.

terig; 12 vielfanmige Sinpfeln - Scauăwu'z, Sempervivum.

\section{Rluffe. S sin oder guevbrubrige.}

Reine Edunterlingobluthe, fondern regelmäig.

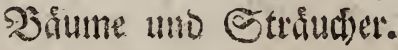

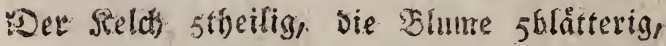
Die Rapfel sfåd)erig, 3fhualig; die Saa: men mit ḩartfonen; 10 Tläget , Tamarišle, Ta-

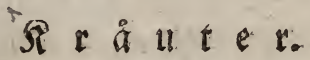
marix.

I Nit 5 Ttågern; Reld) und Blume 5 blitterin; Die Stapiel 5 fohanlig, Iofåd)erig; bie Saamen eimeln = Lein, Linum. 
2 mit to Trigerll, einige bavon zumet: len beutélos; I Staubweg mit 5 ఇarben; Die Frutht 5fanuig, Beid)un belt

3 mit vielen Trägern; bet Seeld bop" Etordidilabol, Geranium. pelt, der cuspere 3 blätteriz, ber ill. nere Iblatteriz, 5fualtig, die Şlume Iblätterig, bis an ben Gimus stbeis lig; Die Siapleln zablecid), Iramigr in einem sireife

§otipel, Malva.

\section{sine Edometterlingatuthe.}

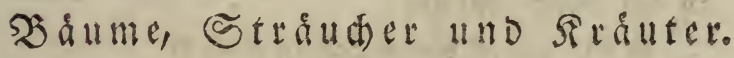

I. Nit 6 હtanbbeuteln; am Birmse jeber Sluttbe 2 Sjlütheanfäzze; ber Feld gefärbt, ttad) Der Eeite, zblåtterig, zlippig, bie Dberlippe ridfwrits in eine SiEfre vers langert; 2 Slumenblátter, mit ben Seld). blattern wedbfelfettig, oben an Ende mits einanoer verbunden, 2 Sriger, jeber mit 3 Seltelir " " " surand, Fuma-

II. Snit 8 Scutelir; ber Seld bat 5 blits ria. ter, = baveu Pligelobulich, geförbt; Die

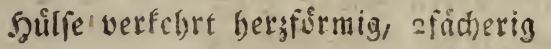

Sitelfystume, PoHI. Nit 1o Etmubgeuteln. lygala.

\section{A. Ille Trriget in Rinem fiórper}

a bie Narbe unbebart

I ber Reld) 5jånnig, baudjig; bie

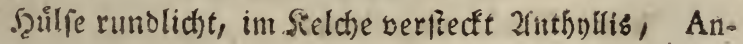

2 ber field) 5 theilig, bie Ibeile (d)mal; thyllis. Die Fubne geftreift; Die Sculfe

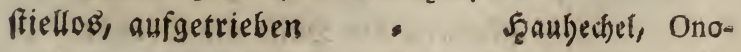
nis. 
3 ber Reld 2lippig; bie Dberlippe

2- Die untere 3ipaltig; bie Trabne

långlid)t, vom Stempel abwårts gebrúçt

b bie शarbe zottig, bem Enbe beß Ģrif: fels an ber Seite Der Rånge nach) angewachien; ber Reld) 2lippig Pfriemen, Spar.

B. 9 Tråger verbunben, I fren).

હinfter, Genifta.

a bie शarbe' gebartet

a ber Relch 4fipaltig, bie f̧úlfe lang gegogen s

Fobinie, Robinia.

$\square$ ber Relch 5 ipaltig; tium.

aa bie zween obern Bågne fúrger,

I breiter; ber Griffel zfan. tig, oben mit einem zottis gen Riele : = Erbfen, Pifum.

2 ber Briffel flach, unten brei: ter, oben fottig - Platterbfe, La-

3 bie Rarbe an ber unterfeite thyrus. gebartet " "

4 bie Şưlfe mit Suft aufgcblas ren $=$ Colutea, Colutea.

bb bie zween obern Bånne tiefer abgerontert; bie Relchmůnoung

rhief; ber Giriffel linienformig Bergerbfe, Oro-

c ber sieldh zlippig; Ctaubgefäge, bus.

Biriffel unb Schiffden idneden: firmig genunden : : Sognen, Phafeo-

b bie গ?arbe ungebartet; lus.

a ber Seld alippig

I bie Dberlippe 2- bie untere sipaltig, bie Şúlfe am Girunde bưnner, faft geftielt . Cutifug, Cytifus. 


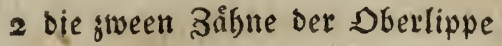
jupammgervachen; sie Fabme Eaum länger als bie Flügel, bie Scullfe verengert " Sronenwidé, Co-

$b$ ber Sieldh 5theilig; sie Ibeile ronilla.

gleid), ro lange als bie Slume Einie, Ervum.

$c$ ber Sieldh 5 fpaltig

I bie Şuilfe 4fantig, ober faft malsenformig, inwentig unter Den Eaameit mit unvollfomme? nen queerlautfonden Sdjeides mainben befergt; Die Sanmett faft walgenferemig

,

2 bie Şuille faft im Sieldje verffeckt

t bie Reldbånne lang; ber un: terfte am långften

,

Rlee, Trifolium.

† bie Boifne furz, ber unterfte ant Eurryeften

3 bie ḩưllie 2fâd)erig; bie Sdjaas

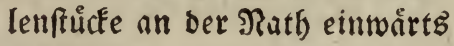
gebogen

4 bie J̧ưllfe gebogen; bas Schiff: M)efifotug, Melilotus.

Tragant, Aftragalis. dhen yon ber fabme gerabe wegftebeno :

5 bie f̧ưlle fid)elformig

t fulammgebricfit, an ber ei= nen Seite einigemale aub: gerandet

\pm runblicht

6 bie Spitze bes Sahiffichens fatief Şufeifen, Hippocrepis.

গ̇ogelfuß̇, Ornithopus. abgeftukget 
I bie Şứlfe gegliebert, febes Gilied Ifaamig eúfitee, Hedy. farum.

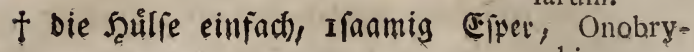
chis,

\section{XIII, Rlaffe, Mefrerbriberige.}

Siduter.

Die Tråger in 5 Partbien; ber Relda 5íaltig; ber Rand ber \$̧lume 5tbeilig, ber Grunb um bas mitttere Eăuldyen beraufgesogen, baffeltbe einfúllend; die to Srăger paarmeire aneinanber und mittelfî eines Gerabbangenden Stiels thens ant Säuldthen inner ber SBlumen. baut befeftiget, und aubeinander fregend Geibenpfanges

2. Mit ģabltreiden Trågen, weld)e haarfó: Afclepias.

mig, am Brunbe veriftiebentlich mits pinanber verbunben find; ber Selth 5fpaltig; bie \$llume 5 blåtterig " Johannib́rnut, Hypericum.

\section{Strå $u$ dícr.}

Die Tråger in 6 Rơrpern, auf jebem 2 Staubs tafeln; Seldh und Blume 6blätterig Gauevoorn, Berw beris.

\section{XIV, Rlaffe. Die Staubbeutel verivadben.}

\section{Siråuteir und fleinere Etråud)er.}

t Lauter כawitter.

* Eauter Shalbblůmdiden.

1. Der soben nactit
a bie Samen alle fromenlos

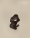
Raintohl, Lape
b- geftoont. fana. 
a bie fraarfrone gefiebert

I fieflos: Der Feld bennabe malgens formig, bie Edhuppen alle aufges rid)tet, bachjiegelformig , Römenzahn, Leon-

2 bie Seldhichuppen in 3 tro concens todon.

trifchen Reiben, faft wegftehent, fo lange, ober auch länger, alz bie blímchen

Boctzbart, Tra.

3 ber Sield) walgenfirmig; bie Sd)us: pen ungleid) bad)siegelfórmig, lan, ietforrmig

1 bie Şaarfrone eimfach gopogon.

Ecorgonere,Scorzonera.

I geftielt; bie fieldorduppen unglcidh, sie obern aufred)t angeorudft; bie untern faft einen ఇebenfeld) bil. Dend

Foraxacum, Ta.

2 Piellos; ber Sield) malsenformig, raxacuin.

etmas eyåntich); Die Schuppen alfgerichtet, oachsiegelforming

3 ber Sielá) Égelfớrmig

an tie Sdluppen in mebreren গeifen = : =

166 Die Ectuppen in jwren Sireis

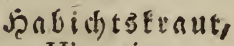
Hieracium. fen, bie innern långer, bie älB̈eru viel fưzzer , Elepis, Crepis.

4 ber Reld) walgenformig

aa bie Etriffe ungletd), badfsiegels

fortmig, breit, lanzetfơrmig Salat, Lactuca. bb ungeråhr mit 5 Schuppen; et: ma 5 Slutitben Se fenftraudi,

5 ber Sielch róbrenforrmig; bie in, Prenanthes. nern Schuppen långer, am Sirunbe ein Eleinet Nebenfeld

Egonorille, Chondrilla. 
II. Der Soben fpreuig;

a bie Eaamen mit einer Searfrone, Sauf́raut, Hypob - - nacft = " Eidjorie, Cicho-

III. Der Soden zottig; bie Saamen mit ei. ner J̧aarfrone - 3úllidf,Andryala.

* Eauter róhrige Blưmchen.

1. Die Slůthen fopffơrmig

A. Die Scaarfrone einfach

a Der Slitheboben bebaart; bie Reld). (c)uppen mit badenfórnigen Stacheln Silette, Arctium.

b Der Slluthebosen mit Sorffen befeşt, bie Sielchichuppen mit geraben Spi= hentr

Diftel, Carduus.

c ber Sllutbeboden ipreutig, soer mit Borften befest; bie âuberften Reld). (d) uppen blattformig, gefiebert, bie innerften bandformig, trocken, raus ichenb, ftralblumchenformig , Eberwur, Car,

d Der Slútheboden mit 4ecfigen Sellen; bie Saamen 4 fantig lina.

Selfentinut, Onopordon.

B. Die ḩaarfrone gefiebert

Eirfium, Cirfium.

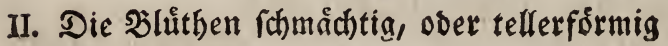

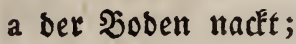

a bie Şaarfrome eillfad);

I ber Selch balbfugelfơrmig, bachs żiegelfôrmig; bie Giriffel Eaum lån. ger, alB bie Sllumchen " Ssoldyaar, Chry-

2 ber Reid walsenforrmig, am Grrun. be faft mit einem Nebenfeldbe, armblưthig

Deftroury, Cacalia.

b bie. 
b die Saarfrone gefiedert, ber Relá) långlicht, bad)sienelformig, bie Birifs fel faft 2fpaltig, lang : Eupatorie, Eupa-

b ber Soben (preuig; Der Reld) bachsie. torium. gelformig; Die Saamen mit 2 fteif: borftigen Granen - Zwevzajn, Bi-
dens.

† Die Sluthen im Llmfreife von verichiedenem Eie. fhled)te, oder Jiefdylechtlog.

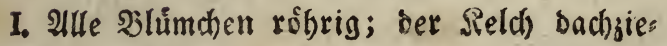
gelformig.

A. Der Slútheboben nackt

2 bie Slithdhen bes $\mathfrak{u m f r e i f e s}$ meiblid)

I faft blumenlos

2 3ipaltig

3 zwirchen ben Reldhichuppen, bie faft gleid), am Ranbe trocten finb; bie Blütbchen ber Scheibe männlich; sie Saamen (oft) fronenlos

b bie Sllithdhen Des Umfreifes (oft) Switter; Die ber Scheibe 3mitter; bie Şaarfrome gefiebert; bie oberften, innern Reldjichuppen rallfheno, geo fårbt

B. ber Slutheboven mit Borftell befegt; Die şlưthden des unfreifes geidhled)t.

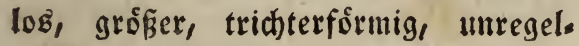
måzig

3

C. Der Sluttheboden bebaart; bie Slúths chen bes Umfreifes weiblich, faft blus menlos
Fadenfraut, Filago.

Rugxp fanze, Gnaphalium.

Slocenolumey Centaurea.

2Bermuth, Abfinthium.

II. ふ่i\&: 


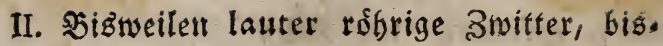
weilen im unfreife faft blumenlos, ober Şalbblumd)en, ber Silutbeboden uadt, bie Saamenfrone einfach.

I Die Reldyichuppen alfe gleich, ro lang alş bie Slumen Şuflattid), Tufi-

2 Die obern Sield)ichuppen gleich, pfries lago. menfirmig, bie am Girunbe nicht io zablreich, fehr flein, Dactsiegelformig, alle an ben Episen verbramnt Jatobafraut, Se-

1II. Sisbrige Zwitter in Der Nitte, im IIm: necio. freife weibl. Şalbblúmchen.

A. Der Slutheboden nactit,

2. Der Sieldh Dad)ziegelformig;

a alle Saamen mit einer F̧aarfrone

a ber Sielch langlicht; bie Echup: pen fpişig; die ångern fưrger, mit megftebenden Episen Jishpflatige, Cam

$b$ ber seldh walzenformig, bie nyza.

Ed)uppen lofe, in mebreren Sieifen $=3$

Iftmannstraut,

c bev Steldh faft halbugelformig; Erigeron.

Die Staubbeutel einfact)

- jeber Staubbeutel unten mit 2 Soriten

Sternblume, After.

$\mathfrak{U l a n t}$, Inula.

c Die Sieldhid)uppen in mebreren Reiben, angedrúckt, Durd)aus gleid)fårbig

Siolorutbe, Soli-

f bie Reldfiduppen in zwo seis ben $s$

23olvertew, Ar-

b bie weibl. Saamen nackit (bie ber amitter nut gefroont) nica.

Scmientwurz,Doronicum. 
c bie Sramen geranbet

a bie Seldhid)uppen in groo Seiben Jinastiebe,Bellis.

$b$ ber Sield balbfugelfơrmig; bie

હd)uppen nidht reibentweife Pyyethrum, Py-

d bie Snamen meder gerandet nod)

getront, wollfommen nadft

rethrum.

Nuterfiaut,

3. Der Reldh einfach, gleidh, vielbli̊t. Matricaria. terig

I die Ganten mit Şaarfronen 2ljhenpflanze, Ci-

$2 \longrightarrow$ Det Eajeibe fers= len, die bes Lumfreifes find frumm, geranbet, $\quad$ Pingelblume, Ca-

B. Der Slitbeboden fpreuig; neraria.

a Der Saame nactit,

a bie Şalbblumdten fparfam-5-10;

Der Selch) balbfugelformig, bacts ziegelformig

Ed) afgatbe,

$b$ Die Şalbblůmdjen jaflreicher; ber Achillea. Shlithebooen Eegelfơrmig

b alle Saamen mit eintem Ranbe ge: trơnt

c bie Caamen bes umfreifez fehlen,

Shamillen, Anthemis.

Tinszange, $\mathrm{Bu}$ pthalmum.

a Die Caamen ber Edreibe mit zwo abfallenden Epiz̧en gefroont; Dev Stelch bachsiegelformig, Der ફlluttbes boden flach

-

Sonnenblume,

- Die Saamen der Scheibe mit swo Helianthus.

Grannen gefrònt; ber Relah viel. blåtterig, Die untern Reld)ifhuppen wegftetyens

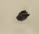

ITs angengefid $\mathrm{t}_{\mathrm{y}}$ Coreopfis.

XV. 2ilatis. 
62

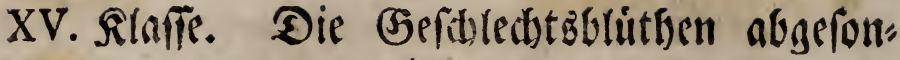 Dert.}

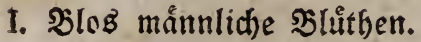

A. Die Etaubgefåze frev

1 mit I Etaubgefåke; Die Slưthe ofne alle Slutthenbecfe, einģeln, in ben 2Binfeln

$=$

2 mit 2 Staúbgefåß̊en;

I ber Sielch eine Iblätterige Sdhuppe; am Brunde eine Drůfe, = 2 Beide, Salix.

2 ber Reld I Ilatterig; bie Slume fibit $=$

Teidflinje,Lemna.

3 mit 3 Ctaubgefåẩen.

a Der Reldh fehlt; sie Slume Iblăts terig, am Sirunde gebogen

b ber Feldh Iblåtterig; bie Slütben in eitrem bad\}ziegelformigen, wal.

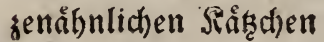

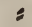

c bet Reld 2flappig, unbewebrt

a sbluthig; bie Epelge mit einer geglieverten Granne; Die Sllu. then geftielet -

Sartgrab, Andropogon.

b 2blutbig; bie Epelge unbewebrt Tilfiches Sorn,

c 3blutthig; zwilchen ben 2 månn:

Balbrian, Valeriana.

Ricoglaż, Carex.

lichen ein weibl. Şlüthdhen; Die Epelzen am アande gefranzt, bie áupere Slappe mit einer furzen feifen, grannenaibnli= chen Borfte

D Die Slume fehlt; bie \$lluthen Sayaftane, Savain einer fegel = ober folbenfor: migen $\Re$ ifpe

Pferogera, Holcus. 
d Der Reld 3 blâtterig; bie Slume fehlt.

a Die Shluithen in einem walgen fơrunigen Siăhchen (Die ঐflanje ein Şalbgraġ)

$b$ bie sblittfen in eimem faft runs ben fiåżdien (ein grabartiges Siraut)

4 Mit 4 Staúgefớzen.

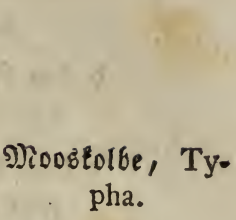

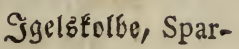
ganium.

a Die slume fehlt

a Der Reldh 4tbeilig; Die Etautbs beutel trågerlog, am Sielche angemadjien

Miffel, Vifeum.

6 ber Sieldh) 2theilig .. Şaftoorn, Hip-

c ber Sield eine zectige Edjuppe હagel, Myrica.

- Der Seldf 4theilig - Niaulberbaum,

e Der Seldh feglt audh = Nerfel, Urtica.

b bie Sllume radformig; ber Sield) 4)̊ånig = = Etedpalme, Ilex.

c Die Şlume theilig; ber Sield

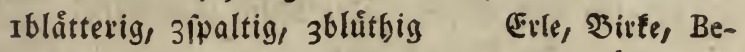

d bie şlume 3 blůthig, 2blåtterig; tula. Der Seldh) 3 blätterig. Fin 2 nfang

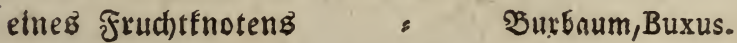

e Die Slume 3-4theilig; Der Seld fehlt. MRit einer Epur eines Girifs fels

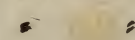

Salantie, Valan-

5 Mit 5 Staubgefáanen; Die Slume tia. feflt.

a Der Reld Iblåtterig, 5tbeilig

a bie Trăger långer, alş ber Relldt;

Die Beutel 2fnópfig - Epinat, Spinacia.

$b$ bie 
5bie Sråger fefre furró ; bie Şets tel 4eckits " Sant, Cannabis.

b ber Selch $3-5$ blatterig; Etaubge: fâfe $3-5$.

2(mintauth), Ama.

c ber Seld 5 blåtterig; bie Trioger

rebr furz, bie Seutel ablang

6 Nit 6 Staubgefåsen; ber Sield) fehlt; ranthus.

f̧opfen, Humu: lus. bie Sllume 6blåtterig. Wom Frudbto fnoten nur ein Infang

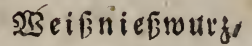

7 Jit 8 Staubgefáken.

Veratrum.

a 3 săme

a Der Sielch 3fipaltig, fouppenars tig, 3blüthig; Staubgef. 8-I0. Scajelftaube, Co-

b Der sield faft 5 fivaltig; bie rylus. Slume fehlt; Etnubgef. 8-12. Eiche, Quercus.

e ber Reld 5fpaltig; bie Silume 5 blåtterig , $\mathfrak{l}$ born, Acer。

- Der sielch eine gerrifiene Echup: pe; die গ̇lume fråufelforrmig, rfhief, gans = " Eipe, \$ałpels

b (5in Siraut. Der Seldh 4 blåtterig; baum, Populus. Die Slume feglt , s Severf́raut, My-

8 Nit 9 StaubgefåBen; ber Reld riophyllum. 3theilig.

a Die Blume feblt; bie Staubbutel Eugelförnig gepaart.

$b$ sie Blume 36 låtterig

$=$

Singelferaut, Mercurialis.

Trojedbin, Hydro-

9 Mit 1o Etaubgefåzen; ber Reld charis.

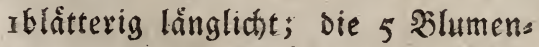
blâtter mit Năgeln; die Platten eins geid)nitten

Eiditiósteiti, Lychnis.

1o গRit 
10 Mit mehr als ro Staubgefånen.

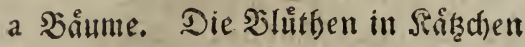

a ber Sield eine Iblüthige $\subseteq d)$ up: pe; bie פlume 6tbeilig - IBalfmub, Iuslans.

$b$ ber Reld) I blåtterig, glocfenfór

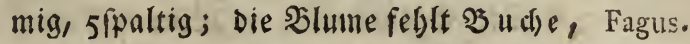

b Riråuter

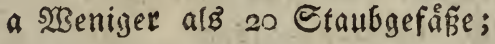
Der Sreich vieltheilig; bie Silus me feblt

5 mebr alв 20 Etaubgefófie,

Şoublatt, Ceratophyllum.

aa aus ber Slume; Der Reld 3 blätterig; Die slume 4theis lig; Die Trăger febr lang, baugend

Sedjerbfume, Po-

bb aus bem Shlutbeboden; terium.

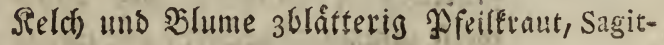

B. Die Etaubgefã taria.

a Sล̊ume

aa bie Staubgefå̉e in Rinem Sions per. Die Slume feblt.

a 3 Tringer; dic Echuppen bes

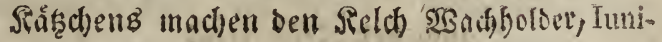

5 vicle Trơger. perus.

a Der Seldh ber Sinope bat 3 ober 4 Ed)uppen; Die Etaubbeutel ichildfork mig, vieljpaltig Sarbaum,Taxus. \$6 Der Jield 4blàtterig Fidjte, Pinus. 
bb Die Ctaubgefábe in mefrern Ror: peru. Der sield cine gefranzte,

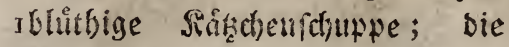
Slume feblt ; Shager beblatifig 10, jeset mit ztween Staubbeuteln

b Sirniter.

Scaunouthe, Carpinus.

aa Die Staubgefáge in Kimen Sior: per

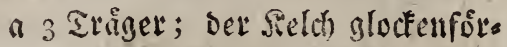
mig, Iblatterig, 5zốnig; Die Silume stbeilig

Sillbiö, Cucur:

6 5 Erriger; Der Seld gemein. bita. (d)aftlid), badjitegelfornig; Die

- Slunte funffpaltio, tridsterfor. mig; oer Slütbeboben fpreuig. Epigktette, Xan-

bb oie Staubgefáfe in melyern Sidr. thium. pern; Der Sield bat 5 pfriemens formige Bâtne; Die Blume ift stbeilig; 5 Iriger; vier war: weife zufammgetwachlen, ber funfte frey onia.

II. Slos weibliche Slitben.

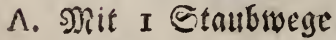

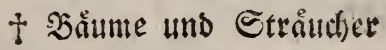

2. Die Sllume feblt

a aud) ber Seld feblt

I bie frudht eine faftige Sees re; (oie Rinopie offret fid) in 3 oder 4 Sduppent

Tarbaum, Taxus.

2 Die Frucht I langetformiges Rorn in eitrem zungenform.

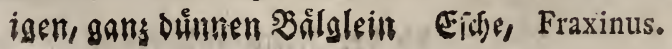


b ber Reld eine Iblätterige 1 blü thige Eduppe mit einer Drůre am Grunde; Der Giriffel 2fpals tig; bie Siapfel Ifädberig, 2idjaalig; bie Gaamen mit ei: ner Staarfrone

SBeide, Salix.

c ber Sielch 2ipaltig; ber Griffel Ifach; bie כeere Ifaamig

Scaftoorn, Hippo-

গ. Die ふুlume birnformig, (d)ief, phae. gan; Der freld cine zerfaliffene Schuppe; die Eaamen in einer Siapfel mit einer Saarfrone

\section{t Sráuter.}

Pappel, Erpe, Populus.

a bie Blume feblt

a ber Reld 4 blatterig; Der Birifs

fel feblt; Die Frud)t eine rian. mige Beere = " Miftel, Vifcum.

$b$ ber Sield) 3 blätterig; bie গars be 2 fpaltig. Eine faftlope Stein= frud)t , s

Sgetátolbe, Spar-

c ber Geld) 2blítterig; Der (Jirif. ganium.

fel 2fpaltig; I breitgebruidter Saame -

Dielbe, Atriplex,

- Der Reldh 4fpaltig; Der Saame

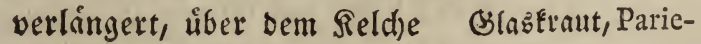

e ber Reld) 2flnppig; I glanjen. taria. Der Saame; Der Ciriffel feblt Neffel, Urtica.

$f$ ber Seld) Iblätterig, e̊fnet fich an ber Seite; bie Siaplel If it: d)erig, fugelfớrmig, mit läng: lichten, an ber einten Seite ges. ftreiften Saamen

Feidjlinle, Lemna.

E 2 g Det 
I Der Seld sieltbeilig; Selch und Briffel feblen; I maciter Saante

J̧o tophyllem.

b bie Bhlume ift 4theilig; Der Relth 3 blatterig. 2แร ber verburtete Shmentofgre ift eine 2fanmige beete geworbes ", " Bedferblume, Po-

c Die Bilume 5thrilig; Der Sield terium. 5 intitig;

I Det Eriffiel 3-4theilig; bie 2arben misgerandet

2 glodfenformis; Der Griffel ลanturite, Bry5ipaltig; bie frudt)t eine Beere; bie Samen am Sian, De aufgetrieben

Sirtbis, Cucurbita.

d bie Slume an ber Mothoung in onia.

5 frumpie Rappen getbeilet, am

Grunde gebogen; der Sielúi fehlt; Der Gamen mar Ein Sorn

t Griffer.

\section{Ealdrian, Vale- riana.}

a Die Blutthen in einem bachsiegelfúts

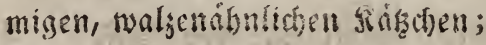

I Der Seld) 1 blinterig; গias ben $2-3 ; 1$ Drentantiget Eaante

Siieograg, Carex.

2 Der Reld) baarformig, jottig; Ein Samentorn, alf cimet banrformigen Eanmenfrome auffirend $\Rightarrow$ Mooskolbe, Ty-

b) Sield) und Blume 2 fdhalig; mur pha.

I fabenformiger, berabbangender Giriffel; Caamen einselin, in beit

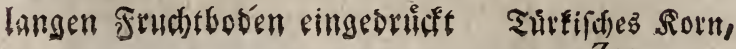
Zea. 
3. Mit 2 Etrubrogen

a Cin Gras. Der Selds aflappig; bie Silapen faft faljuformig, foff gleid)
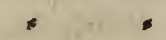

b Srónter. Die Slume feblt

a ber Seld) Iblitterig, gany;

I an ber Seite flaffent; sie Frutht

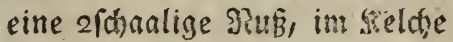
weridyloffen $=$ $=$ Şanf, Cannabis.

2- fotief fich offuend; bie Frudht in Bapfen; Der cintyche Caas me im blitterigen sieldie vers (c)lofien $=$

Şopfor, Humti-

3 ber Ield eine 3 edfige Sduppe; Saveliane, Savaftana. bie fonberbeitlidjen grud te in in eituen beerenilynlichen, Ieber: artigen Bapfen gefanmelt; jebe einfaumig $=$ Songet, Myrica.

1 b ber field 3tbeilig; sie Rapfel z f no:s pfig, 2 fĭd)erig

Singelfiaut,

c ber seld) ablatterig, jwevblitbig; Mercurialis.

Die Eteinfuch)t trocten, fradylig, 2fpaltig; Der Siern 2 fächerig

Epif̧⿻上丨tete, Xan-

c গ̧åume. thium.

a Die Slunte feblt

I ber Reld) Iblåtterig,

aa volffommen ganj, fieifoor: fitig; I enformiger Sanme in aubgernachienen Sieldhe (rid)e, Quercus. bb faft 3ipaltig, zblütbig; ber Saame gefligelt Erile, Sirtfe, Betula. 
ec 2 blüthig, fduppenartig; bie Frucht find Bapfen; in jeder Schuppe 2 gefligelte গRffie Fidre, Pinus.

2 bet selda) 2 blatterig, zerriffen;

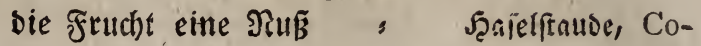

3 ber Reld) 4 blâtterig; baz Sintes d)en wiro zur Jeere . Jiaulberte, Mo-

b bie Şlume 4theilig; ber Selch 4 fipaltig, íber bem fruchtfnoten. Cine trodene Steinfrudtt : $\mathfrak{B a l l n u}$, Iug.

C. Mit 3 Staubwegen. lans.

2 Sล̊̊me.

a Die Slume fehlt;

I Der Reld 2flappig; I Saante Surbaum,Buxus.

2 ber Sielch Iblătterig, 4s̊ănig; ber Sielch wirb jur Raplel, bie

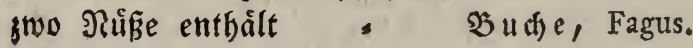

b bie Sלlume 3 blåtterig; ber Seld) 3theilig; Die Seere 3laamig Wadjholder, Iuni-

b Rråuter. perus.

Die Sllume feblt; ber Reld) $3 \cdot 5$ bläts terig; Die Siapfel Ifädherig, theilt (iid) nad) ber Dueere; I Same 2(maranth, Amav

D. Meiftens 4 Staubwege, sod) zumeilen ranthus. mebr, ober weniger; bie פlunte feblt. a Rråtuter.

a Die Bluthen in ben SHinfeln; ber Reld) Iblátteris; Samen fo viele, alB grudbtfnoten a Bannidjelie, Zan-

ber Sield 4fpaltig; I Saame im verbårteten Meldhe nichelia.

Spinat, Spinacia. 
e Det Seldh 4 blatterig; 4 uncfte Samen

Fiderblatt, $\mathrm{My}$ -

b (sin Bautur. Der ßeld) eine Iblis riophyllum.

thige, jottige Siafbchellobupe; Die Silume feldhåfinlidy, fechsipaltig; jwev) Etuicle bavon grî́fer; 2 Frudts Enoten, jeber 2 Griffel $=.2$ Schunbudhe, Care

E. Nit 5 Ctaubmegen; Der Selch Iblat: pints. terig, linglicht ; 5 Slumentlatter, jebes mit einem Nagel; bie \$latten einges fohntten; Die Sapfel sfähterig = Iidtisstein, F. Niebr als 5 Etanbroge; sie Bhlume Lychnis. 3 blätterig

ber Sield) alth 3blatterig; wiele nadfte

Enamen

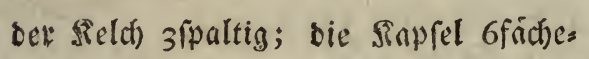
rig, vielfanmig

Pfoilfratt, Sagittaria.

Trolitisis, Hydrocharis.

\section{Silaffe. Uneentlidbe Blutthen.}

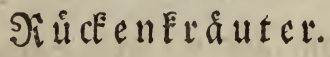

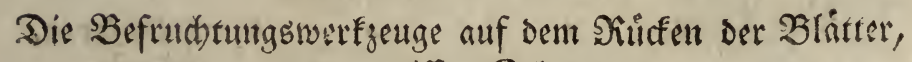
oder gerwiffer Edduppen.

I. Sie Slúthenfduppen in länglichten $\mathscr{A} e$ s: ren, foiloformig, unten mit ben 2 efruchs tungšwerfieugen befergt; Der Stengel ges gliebert, bas Raub im Surt, gegliebert, fabenfórmig = = " andelivifd,

II. Die Frudthe fltgelfêrmin; Der Stengel Equifetum. faft gegliebert, das faub fabenformig, glieberlog, in suirl : a Chara. 
III. Die Rapfel sfnet fich in bie Queere; bie - Hebren zungenformig, zwenfeitig, geftielt; bas \&alb geftielt, ganz = = 9Rattergunglein; Ophiogloflum.

IV. Die Sapfeln wfuen fid in bie Queere; bie Aebren traubenformig, geftielt, einfach Doer åftig; das Rnub geftielt, gefiebert, Doer gefiebert zeríchnitten

Traubenfarm,

Osmunda.

v. Sie Frudtfapfeln

a it puntformige Ђ马æ̊ufchen, reifenmeife georonet $=\quad=\quad$ : Tipfelfarm, $\mathrm{Po}$ lypodium.

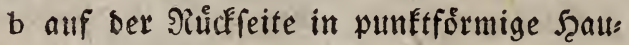
fen reibenweife georbnet, bas blübende faub von bem unfrudtbaren in eben Derfelben Pflange verfchieden

Spicant, Struthiopteris.

c nebmen bie gange Sactifeite bes Raubes, wo fie fitsen, affenthalben ein

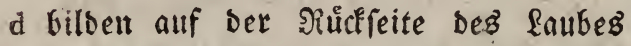
Sollfartn, AcroSirten, bie mit bem Slattrande, unb Der $\Re$ ibbe parallel fieben Sa $\mathfrak{a} \mathfrak{m} \mathfrak{a} \mathfrak{r} \mathfrak{n}$

e bilben Rinien, bie auf bie Mittelribbe rifief freben Pteris.

Streifenfatros Afplenium.

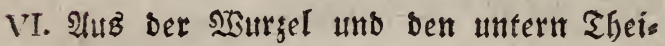
ftichum.

Ien ber Slattifiele fommen evformige Entloe, berer llnierfeite nad) ber Range

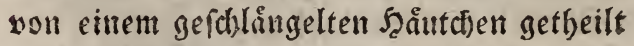

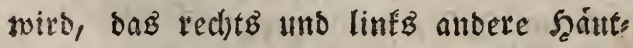

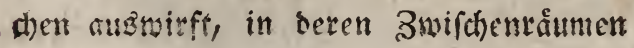
Die Befructungànerf́geuge finb; Das Enutb verichieben; bie Şlutben 3mitter . Dialfitie, Mars filea. 


\section{2no of e.}

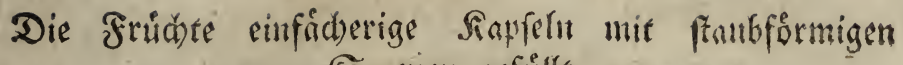
Saanten gefüllt.

2. Die Sappel mit Gleibendem Deckel; $a b=$ fallent

3. Der Nandrand ber Sapfel-

a nadfe $=, \quad=$ feulenfermigen Enden ber obern Zmeige Torfmoos, Sphag,

c baarfein, cinreibin gefranjt; bie Fran num. zen fdraubenformig gemunben $=$, Miollie, Mollia.

d in einer গitibe gefroint

a mit 4 Sronenfticfen = Siel'ashn, Tetran

1 bie zåntue boppelt; ber lunterfats Dhumuno, Phafcuin.

Sablmuni, Gymnoftomum. ser sapfel ben ber frudstreife ver: febrt fegelformig aufgetrieben, oder butformig erweitert

c Die I6 Sironenftude phis.

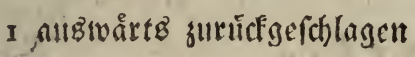

a priemenfórmig

B oreyedformig

2 nid)t zurlicfigefdlagen, gans

3 oben zweyrpaltig

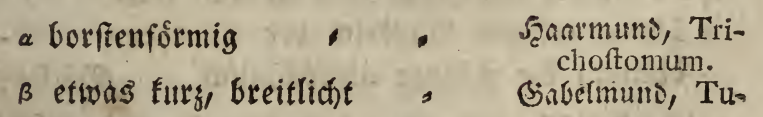

b 32 Stronenftucfe; bie Epitzen mit eis uer Şaut verbunden

e in stwo Reiben gefront, bie âußere Reibe if mit 16 ล̊ăhnen verfeben. (c) it minsos; Splachnum.

Eecrifie, Lcerfia. Birimmie, Griminia.

Vseifie, Weiffia. fcina.

STsibertfon, Polytrichum. 
a bie I6 Zånne fpişig; bie innere Sicibe

I mit einformigen Fransen,

+ verbunben burd) eine ges meinichaftliche Şaut aut astie, Leskia.

† ganz unverbunden $=$ Neckete,Neckera.

2 mit verf(t)ieden gebildeten fralt: zen aus einer gemeinichaftli, chen fraut = "2 2fimoos, Нyp-

3 mit einer şaut mit ober obne Framen $=$ = sisebere, Webera.

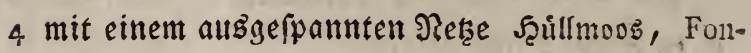

1 bie I6 Såhne an Den Epiţen an= tinalis. einander bangens; bie inmere Seife mit I6båutigen flachen వàt): nen

c Die 16 3irithe abgeftutrt; bie int Solreute:c, Koelnere Nieige mit einer faltigen Saut

burteaumie, Buxbaumia.

\section{$\mathfrak{A} \mathfrak{l} \mathfrak{g} \in \mathfrak{n}$.}

a Mifroffopiich Elein, moosånnlid), unter: getautcht, mit febr langen, gernden, baarformigen, burchfichtigen Stralen Scaarmoos, Chae-

b bie Raprel 4fhaalig, fitellog, reif klafs tophora. feno, in ben șinfeln ber Slüthen= blåtter; bie Pflange moobånnlich Sårtapp, Lyco

c bie Rapfel 4fạaalig, reif Ereusformig podium. aufipringenb, meiftens geftielt; die Stånme friechend, bie Sweige oft auf: gerichtet, ober mit Blattern bebecti Sungermannie, Iungermannia.

d Cin 
d Ein Slatt friechent,

I lappis, aus demfelben fins Sticle mit cinem Sçute an ber Spitze alls: geridytet; Die Slüthden und Stapfeln auf ber Unterfeite Diefes feutg

Mard)antie, Mar-

2 fett, mach Der Ridftung Der Nerven chantia. Iappig, aus bem Enbe Der Nerven ein Gtiel mit fuglichten Fruidten an Der Epiąe in einer ftiellofen Iraube 3 Infie, Blafia,

3 etwas fett, auf bemfelben becherfers mige Erfoofungen, unb aแs biefen pfriemenformige Etiele, bie reif in zwo Echalen berften, unb den Ean, men ausfduitten $\quad=$ Sgornetiojorf, An-

e långlichte, burchfichtige, bullfenånnliche thoceros.

Gebñure mit 8 Durdrafheinenden Ean= men, Der Ctrunk feblt oft gan's oft fehr einfach, worauf idhifitelformige, tellerformige, oder polfirige Seirper, dic eine unendidje Nenge nufgeridhteter Saamengésurule smirchen nutliegenten unverbundenen Fioden enthalten

Id ditinamen, Oeto.

f eine meblige, oder fruftenformige, oder fpora.

fabenformige, ober blatterige, obet ei: nem falfartigen Strauche åtuliche, oder båutige NFange, mit Ssargen, Netht. flimpchen, Sinpitelldyen, Edjưfleldhen ober Siechern . " Sledte, Lichea.

s lange, aud trocken, biegrame, bolsige, oder faft hornartige, åftige faben, obne alle Epur von Frudfifification Niwhaar, Usnea.

h ein bloffer vegetirender Gtaub ofne Edjüfieldient
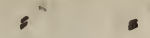

,
Staubmoos, Lepra. 
i feine magere Satre, wie Cammet, obne

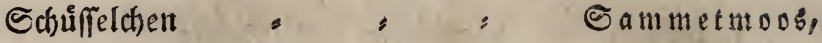

k Gaarformige, inwendig mit Şlíschen angefülute Fåben ",
in einem unaufioglichen, borftigen, gals

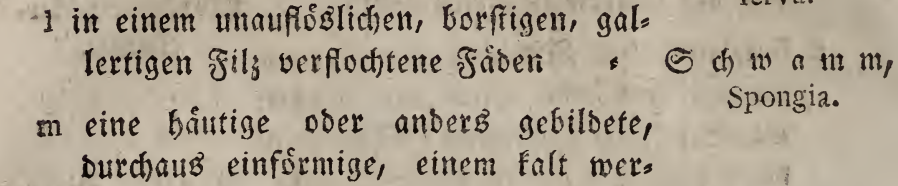

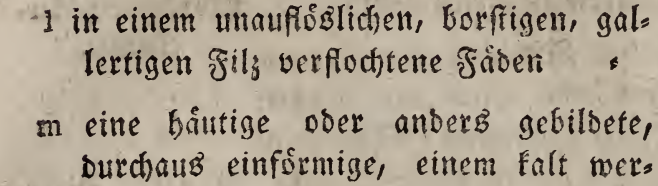

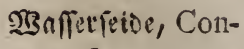
ferva. bensen feime ânliche, elaftifhe, troden zufammenforumpfende, in ber geud). tigfeit fich rebr aubbefnende Pfange. Sieine Spur woit Frudttbeilen, Siallerte, Tre.

n eine burdfideinende, rogrige, auffer Spongia. bem $25 a f f e r$ gang zufammenfallettoe, tro. dfen gan jufammenfdrumpfonde Szaut, ofne fructitbeile Ulve, Ulva.

- eine fugelformige Garte Shange nit eis net meblatigen, oft trodinen grafie angefurlt Siugetpilf, Sphasria.

$p$ ein feiner weiffichter Etanf auf ber mella. Sberfiad)e ber Breige, oder ein born: artiger, getweihformiger Sorper, an ben Enoen mit feinem Staube bereift Fingerpil; Xy-
laria.

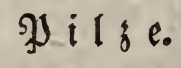

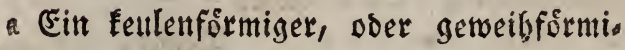
gev, Didhter, zấber, fofmieriger Wils mit feinem Saamenffaube bereift

b ein leberartiger mit ber Unterflåde ans Seulenpilz, Clavaria. getwachiener, auf ber Dberflakd)e aberi. ger $\mathfrak{P}$ ils

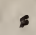

2oeinpilj, Merulius. 
c ein aufredter Eticl, mit einem differ abgerunbeten šósidjen, bie Fruftificu, tion auf bem Sespedden idsmierig

Sinepfpilz, Acro*

a ber faut am Ende bes Etrunfes fopfs fpermum. åbulich)

I an ber innern Eeite glatt, an ber

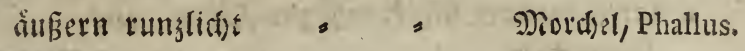

2 ben volliger Reife gitterformig, Durchbrochen

Eitterpily, Cla-

e ein werfóleben faltiger \$ils, beffen kuls pere glähe mit einem Eaamenreife bes follagen ift " " $\quad$ Faltenpitz,

f bie lunterfeite bes f̧uteg

I mit frralenfơrmigen જ̧lâttern

2 mit untereinander verbundenen so̊n: ren

3 mit weiden Etacthln, bie mur am Srunbe verbunden find. Etauthelpily, Hyd.

g ein bedherfórmiger \$il 3 , nichts Der vori, gen

h Etautbormige Eaamen, Die ber oben

Ṡlittetpily, Agaricus.

Lóderpily, Boletus. num.

Bed expit Peziza. alfipringende Sils auştrent, ober it feinem Jnnern eutbålt

i ein wedelformiges f̧âptchen, bas bie Etaubpily, Lyco. perdon. ftaubäbnlichen Saamen trågt, ein end formiger bưnner Secher, Der in ber S) itte nach ber Suecre abipringt, uno DaB Şåuptchen blof́ fitellt

Solbenpilf, Ste.

s ein geftieltes tropfenåbnliches, Durch) monitis. (c)einise รูåuptcden

Edlaudfpily, Af cidium. 
1 eine Slafe mit wafferiger feudtigteit gefuillt, mit cinem balbfugelformigen Scute gededft -

Şutwerfer', Hy-

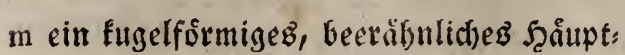
drogera. d)en, bas feime reifen Gaamen in fleis nen bichten $\mathfrak{T}$ råubchen fammelt

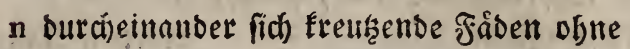
Sujimmel, $\mathrm{Mu}$.

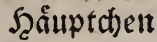
$=$ פ):00ev,Mucilago.

- ein fwinnengewebartiger, zwifh)en Den Fingern zerflieflender \$ils.

SBettergotte; Ly. cogala. 


\section{II. $\mathfrak{I} \mathfrak{b} \mathfrak{e} \mathfrak{i} \mathfrak{l}$. $\mathfrak{A} \mathfrak{i} \mathfrak{e}$}

5. Abfinthium, șermuth.

2 Iaufend gefiedert; Die Ctucke lanjetfór: mig; Die Slluthen faft fugelformig, ein. reitig (bılb). Juli, 2lug. Wobn. ungeb. Srte. Grbr. Epeife, Nedicin. Farb.) vulgare, semeis

b bie Seitenftamme auffehend; bie Stengel net.

unb פlătter filgig, grau; bie Slâttchen 3theilig; die Stúdfe linienformig, lang (

\section{Acer, 2Iforn.}

2* Die Slåtter fünflappig, ungleid) fågę̧åh), nig; Die Slluthen in Srauben (WOLn. in

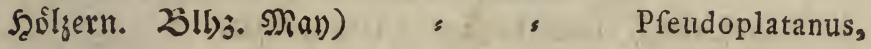

b fd)arffpizing geşånt, bie \$lưthen in einem sueiß̄er.

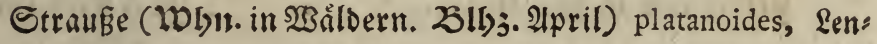

c bie Rappen fumpfectig (WWlit. in Scedfen. ne.

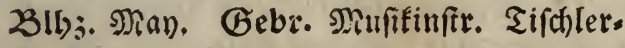

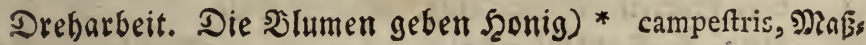
bolde.

\section{Achillea, 2(bjillenfraut.}

a Die Slätter fahmal lansetfơrmig, fringig, fein figezainnig (LDLn, an Straffen. 2153 .

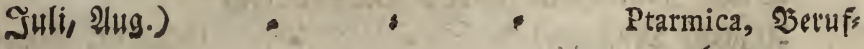
fraut. 
b bie Sllitter gefiebert jeridnutten, von aufs liegenden Scaaren grau; bie Etuffe vors wairts gerid)tet, am (Fnte eingefdnitten; ber Stengel auffétend (MInu. Siebirge. 23ly;. 2ug. Septo.)

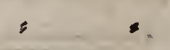

c bie Slätter in foldmale, fpiţige, wenigges

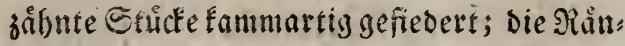
Der ber אeldjituppen verbrant (Wobr. Gebirge. 215: Juli)

d bie Slitter boppelt gefiedert, glatt; bie Iezten Blättchen 3-5ipaltig (10bu. Strafs , Fen, Aecter, SBiefen. Zlbj. Sommer. Gebr. Sctlungșittel) * =

\section{Aconitum, Sturmhut.}

a Die Vlătter .5theilig; bie Etůcfe feilfós: mig, vielfpaltig; bie $\mathfrak{B l u}$ then mit 3- 5 liaps feln (Wolgn. Sobgebirge. Zblbj. 2iug. Sept.)

b Die Silitter vielfach jevtbeilt,
C 1 a ve in na e, Strintaute. atrata, $\mathfrak{B}$ ergmuts terḱtaut.

I Ganofermig, etwas bebaut (TVhn. S3åls ber. $2316 ;$. Jull. Gebr. wiber Unge" jiefer, und in ber 2tpoth.) * " L y c oct onum,

2 bie lesten Studfe lang uno fhmal, mit Millefolium, Sdyanga:be.

Cammarum, lidjt: blauet. gerbet. zitemlid) parallelen Seiten (WOLn. ŞBâl, Der, Gå̊rten. 23lb3. 21tg. Gebr. Nies bicin, fonft (c)

\section{Acorus, Calmus.}

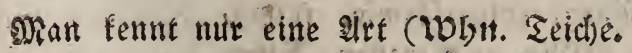
214). Juni, Jutt. Gebr. Standtwein,

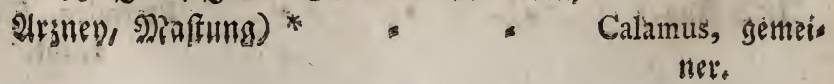


Acrofpermum, Sinopfpils.

1 Slåtterpilfåanlid), intwendig hobl, mild rafmfårbig, leberartig, trocken : ficcum, trodnet.

2 Ed)mierig; Der Siơrper ziemlich glatt, runglic)t, f(h)mutigig roth, bohl, Der Siopf flach, weişlid) (WOln. bebde auf alten Eto̊cfen)
Acroftichum, כollfarren.

Die Strunfe zmenmal gablig; die leste Gabel ungleid); bie Stridfe linienfors.

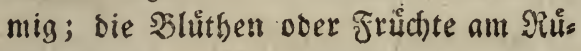
cfen bebecft (Mbur. Felfen) : reptentrionale,

\section{Actaea, Éfrifopfofraut.}

Die Traube enformig; Die Frichte fino Seeren (Why. Doralpen, auch) Fleine

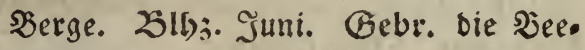
ren geben Dinte) * = = fpicata, gemeines.

\section{Adonis, 2 loorits.}

1 Die Slume 5 blătterig; bie Frichte en= fớrmig

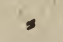

$=$

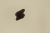

2 bie Sllume eblatterig; die Fruedte faft walzenformig (Whn. bende auf Aectern. 25lb). Juni, Julii)*

3 Die Slume ůber 12 Slåtter; Der Sten= gel åftig, Iblutthig; bie Hefte unfrud)ts

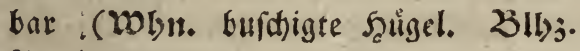
April, Mar))

\section{Adoxa, Sifamfraut.}

aeftivalis, Som. merabonis.

a u tum nalis, Sertofradonib.

vernalis, Fruif. lingšadoniz.

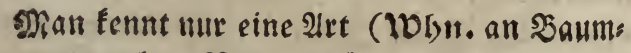

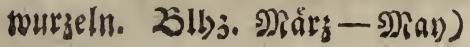

mofchatellina, gemeines. 


\section{Aegopodium, (3ier.fh.}

Die oberften Stengelblåtter brenfingerig

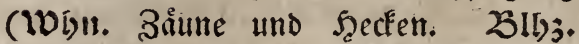

Suli-Septb. Gebr. Epeife, f̧onig)* Fodagraria, 3ip:

perteinftaut.

\section{Aefculus, Jioffaftanie.}

Die Sluthen fiebenmânmig (Whit. wirb

fehr băufig gezogen. 3lb;. Niay, Jumi.

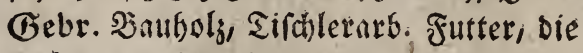
\$lütben geben fronig) *

Hippocaftanunit; gemeine.

\section{Aethufa, Gileiper.}

Die Blatter breinal gefiedert, eingefhnit: ten; Dié Dolbett aus ben SIattwinteln

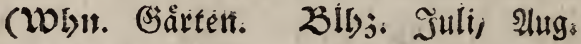
Gebr. (d)ådlich)

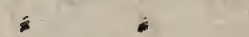

C y n a p i iting,

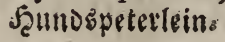

\section{Agaricus, S̉ldttetpily.}

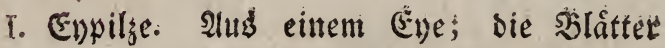
weî́; ber Strun mit cinem Ringe.

\section{A. Det Şut fladi)}

21. am Rande geftreifit

I oben grau, weifsgefledt; ser Strunt weif, Did)t, oben mit einem meif: reir, geftreiften Singe (Wolnt.

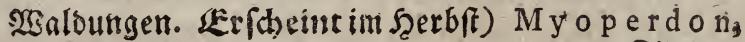

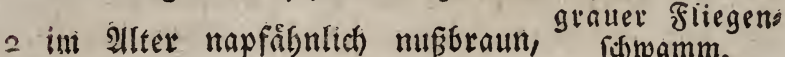

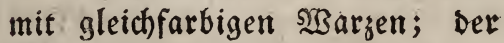
Strunt walzenfờrmig, bich)t, meiß̄,

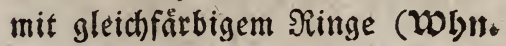
faubbólser, Rerid, im Suli)

(i)tramm.

fubobfcurus, nuf brauner fliegen: (i) 
3.. Reibenåbnlid, blaäitrobgel6, gegeh Die Mitte flevig; Der StrunE wal, zenfórnig, ziemlidf glatt; Der ßing

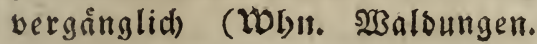
(Exid). im Serbite)

B. Der Scut genooltat,

furfuracetis, flitijl: get Eivpilz.

a, grall, weifrwargig; Det Strunf ab́. wairts bicker (Tolntr. SWaldungen.

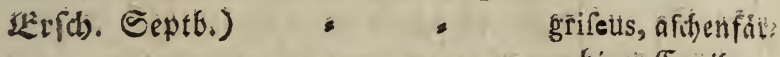

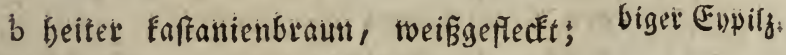
ber Strunk unten Eeulenformig, oben

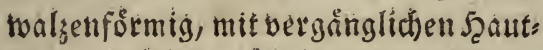

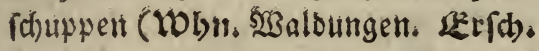
Scerbfit)

C. Der Şut Eegelformig, obent roth mit

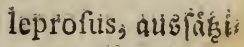
meiß̂nen Şarzen, ain Silanbe geftreift

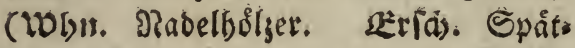
(ommer) , mufcarius, totbet

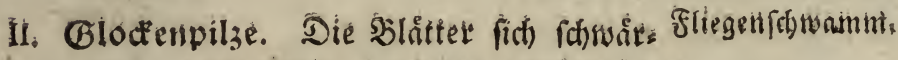

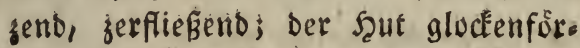
mig; ber Strumt xộrig, walsenfơrmig

A. Weifis;

a ber b̧ut geftreift,

a verengert; bie Slâtter pebr zallt,

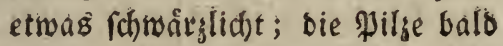
cingeln, balo gebåuft (WDbur. int Gärten auf bretternen Einfaffun

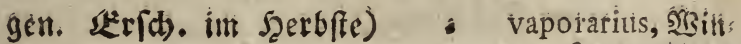

b) fleifhlos

I faubfárbig, anfånglich enfờ tet = Sęaitspaboen. ftul. mig; bie Slittter meifí; bie Silje gebăuft (Wolnt. an Straf

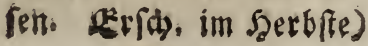

ovatus, eiffortint

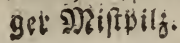


2 jottig, grau; bie Slåtter grau; bie Silge gefellig (wobur. auf beftaindig feuctem 5̧olge) nanus, వwergglos

c frumpf fegelformig, denpily.

I weifllicht, feingeftreift; die Siląt. ter weif́; ber Srumf am (irun: De getumben, bicfer (lang); mebrere \$ilge nacbeinander, boch abgefondert (Woln. an ben Einfaffungen Der Giarten. betten. XErid). in Setbfe)

2 glatt, aidhengrau; bie Slátter vom Strunfe entfernt, weifi ; ber Strunf binn (lang) (10bu. unter Den giosfen)

$\mathrm{m} \mathrm{u} \mathrm{fcorum}$

braun; Der Gtrunf glatt, nacft Moosglocfenpily: (whi. unter bem Giefträud)e. (Exfd). im Mian)

b ber Scut ge(d)uppes, fleifhlog,

extinctorius, Qdichbornformiget Silăttetpilz.

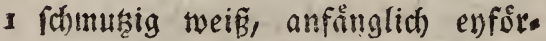
mig; bie Şläter fattid)warz; bie Pilge ziemlich eimgeln (Whit. an Giebrifden neben ben Etraffen.

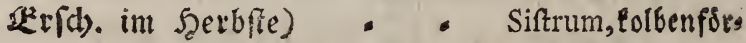

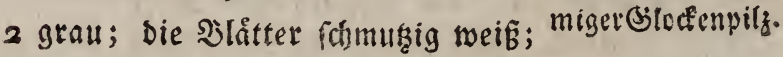
oie \$ilge eingeln (WDLn. Nift. betten aus Gerterlobe. 纹rfh. im fruiblinge) = : vaporariorum,

B. berbleidst roftbraun, geftreift, unten fruticum, ¿̋jifj. glocfenpily.

Qobpasoenftul.

Dicfer; ber Şut etwas fieifhig roftgelb,

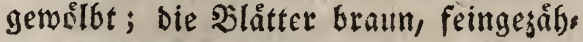
nelt (WDhn. auf Sergen. KErfd, iu Serbfte) 


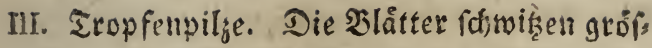
fere, ober fleinere Tropfen (ichimmernbe Puntte);

a bie blåtter ungleich), weislichtbraum, feid) ; bas Fleifh weif̃; ber StrunE Did)t (MVhn. an Etraffen. Erid). im Scerblte) . ".

b Der Wils. bolftrig; ber f̧ut forwiat (d) warge Iropfen (Why?. an Fid)ten. ftimmen. Lerrab. in Eeptb.

hydrophorus, ges meiner Tropien. pils.

Melaclaeon, Din: IV. Fekenpilze. Die Dberfette bes Shutes jerrifien.

1. Der Etrunf ofne Stinge, bicht

a volf; ber frut fleifdig, gelblidbt, mit Feifhfårbigen Eduppen; bie Slats

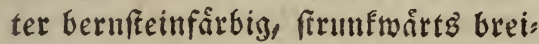
ter (IOLn. in Soghlwegen. 代rid). im Szerbfte) =

$\checkmark$ geftreift; ber Scut flach, an Sanbe gewólbt, Dunfel faftanienbraun, Elein, calophyllus, findurblattrizer Se:

हुenpily. (A) uppig; Die Blîtter bellkoftanien=

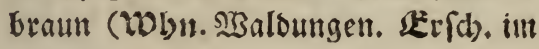
Serbfie)

$B_{0}$ Der Strunf mit einem Singe, tenín wisenoer Fiopfenpils.

fucefcens, bidiult:

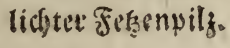

a Walgenfertmig, geftreift; ber ?ling bråunlicht; Der F̧ut gewólbt, rơth: licht braun, geflodft; sie Slätter lichtbraun (WDLn. auf alten Stơcken. Erfd). im Şerbfte gebiuft) . Irus, flocfiget ges

6 hohl, gef(f)uppet; ber Sing meí̈; ह̧enpily. Der Şut leberbraun, geichuppet; Das Jleich) menig (Wobrin. geiellig auf als

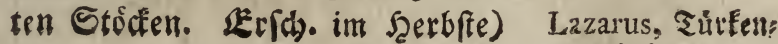
táubling.

V. Tล̊นต: 


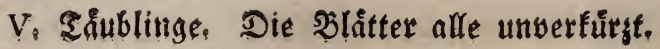

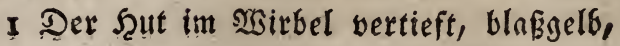
etwas wargig, filjig, am Rande ge. ftreift; Die Slätter gleichlang, mild). tahmfärbig; ber Etrunf nacft, bicht, aufwairts bicker (WWhn, in $25 a l d u n g e n$, Lerfd). im frerbfte) " " integer, gang bläta

2 ber feut fleifhig oben roth, troden; bie teriger Taibling. Slätter faft alle gleidflang, weiñ ; ber Strunf rundlidt, bid)t, mand)mal an (5i: nem Ende sicfer (Woln, in $\mathfrak{S a l d u n g e n . )}$ r u b e r, rothey

3 Der F̧ut leberartig, telferforrmig, im ₹åbling.

Wirbel vertieft mit Neblfaub bebect'; Def Strunf Eurs (Mo)n, auf alten Sitchtenftódfen)

vernus, záfes

4 Der Sut balb fugelformig, oben meblig, ₹åubling, im Wirbel vertieft; bie Slåtter alle gleidhlang; ber Ctrunf fury; bie \$ilge gebåuft, leberartig, braun (10hn. auf

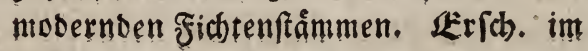
Frůblinge) , farinofus, meflis

VI. Pfifferlinge, (STeiftens Mildagebende get Tåubling. \$il $\mathfrak{e}$ )

A. Mildsende,

2 Die Nild weín;

a Der Şut flach), fleifhfợtbig, glatt; Die Slâtter blakroth; Der Etrunt walsenformig, glatt, of ne ring (Whn. in $\mathfrak{W a l b u n g e n ~ e i n g e l n , ~}$ QEvidb. im Sctb.)

\$ber f̧ut rofffárbig mit nieberlies lactifuus, Breits genden Botten, in sirirbel ver. tieft; bie 'Slitter gelblicht; bet Etrunf bohl (Woln. unter Fid): sen. $\mathfrak{L} \mathfrak{x}\{\mathrm{d}$, im Septb.) 
c ber frut flach, ober vertieft; Die Şlatter riftig herablaufeno; das

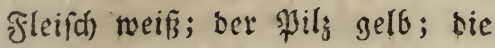
Nild) wenig (20 hrs. in 233 alduts gen cingeln uno gefellig. 位rifb. ium S马erbfte)

Cantarellus, ge:

- bas fleifh mildurabmfórbig; ber meiner Pfiffer

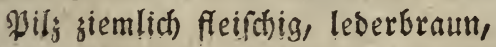
obne Strumfring (IDbur. auf ftei: nigen şiefen eingeln, uno fwars ram gefinuft. UEtifb. im Eeptb.) fufcus, braunet

b bie \$lild jiegelfärbig; ber Scut balb: Táubling. fegelformig; Die Slitter ineinanber laufeno, etwas herablanfeno; das

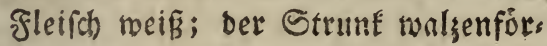

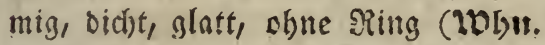
in 23 oloungen. (ERrd). in Şerbfte) cinnamomeus, c bie Mild gelblidht; ber Şut grof, fimmetfárbiget Didé, flebrid)t, alatt, gelb; bie Slät: Jiffifuting. ter gelb; bas Jleifh weiß̄; Der Strunf bick, fur; mit cinem weislich)s ten, lócherigen 2 Befen bebecft (WDIt.

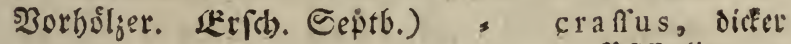

d bie Milla faframoth; ber \$ity rofts Pfifferting. fårbig; Der Scut vertieft; Die linns gerit Slátter etwa berablaufend; Das fleîh weif; Der Strumf bidht

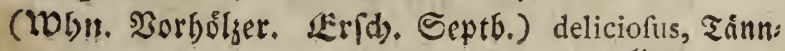
B. Nild)los, Łing.

a Der \$ily meiś; ber feut flact), ober vertieft, am Sanbe feid)t geftreift; bie 5 laitter dunne, Die lángern bers ablaufeno; ber Strunf did)t (WOLn.

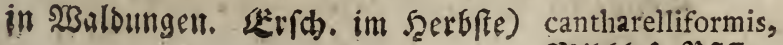
9)il(t)loier'spiffer: ling. 
b bet faut bertieft, fleifhig, am Ranbe geftreift; Die Slátter bicflid)t, une gleid), weif ; ber Strune weif́, nadft,

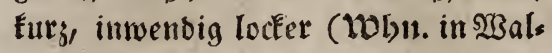

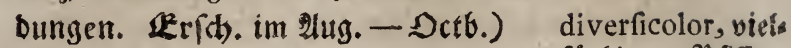
VII. Siragenpilze. Ein bleibender Rragen, uno nidhts der worigen.

a Der f̧ut gewolbt, fletidsig, gelbbraun;

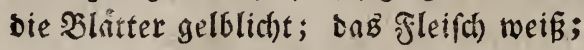
Der Strunf ưber bem Singe glatt, un. rein weifi, unter ifm fhuppig, balb rófrig, balb woll (Wolnn. auf mobern. Den Stơcken gefellig. (E)

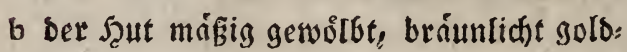
(id) gelb; bie Siătter reingelb; ber StrunE walzettfórmig, bicht, glatt, etwas ges ftreift; ber siing gelblid)t braun (WDlsit.

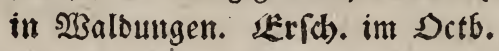

c ber Shut etwas gepolftert, etwas fhups fårbiger Pfiffer: ling.

pig, bóutig geranbet; bet Etrunf faft woll, unten bicker; ber \$ils burchaus weî́ (WWln. auf bergigen Aectern. XErifb. im Sctb. nad) Regen zaflreid), aber einjeln)

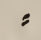
genpilf.

viII. Etthblîtterige Pilze. Die Blơtter rei. fulvefcens, gorts. gelflitgtet. (hen nicht an Den Strunf, weldher glatt iff.

a Der Şut fanft geweilbt, glatt, gelbbraun; bie Slátter merben bunfelbraun; ber Strunf walgenformig, oben geftreift,

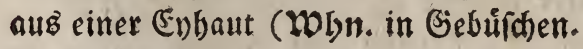
《erids. im Man)

abbreviatus, yer: fủrzłt blîtterigek Stukpily. 


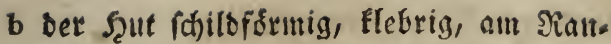
De etwas gefreift; Die Sllitter gelb. Iid)t; ber CtrumE zufanmengedruidt, nacḱt, rébrig (Woln, am Fufie alter Beftråtche. Xeridh. \$?ov. Dec.)

c ber Şut ftumpf Eegelfortmig, nuffárbig; ferotinus, fpaitec bie SIlätter weifi, erróthent; ber Strunf

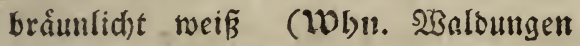

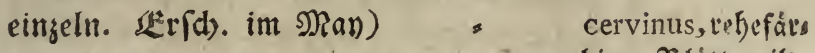

IX. Dưrrlinge. Der f̧ut fleifchlog, oft burd), biget Blătterpils. (d)ẹneno.

a Der Scut fatwach gemólbt, thonfiñbig, mit aufgetriebenem 2 sirbel; bie $\mathfrak{b l a ̊ t s}$ ter meifí, ber Strunk weißß, thonfärbig merbend.

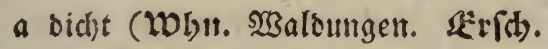
im fribling) = clavus, Nagela

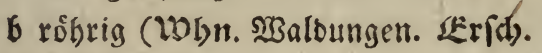
im ๔onumer) - . efculentus, r\$g.

$b$ Det frut gerwólbt,

a in ber Folge tellerforunig; bie Slatter breit, ber EtrunE bohl; ser Pils Durdials leberbraun (Whltr. in Erlengebuifhen. Rerfbs. im Detb.)

alnetorum, Erlen,

brothbraun, wie ber róbrige Strunf; riget ? a ge fo (⿻)丨 1 a $\mathrm{mm}$.

bie Sllitter meifß; Der f̧utranto zuridfigerollt (Woln. faulende (Fi, d)enrinde. (Erfid). im Sammer) convexus, gcwólb, c Der F̧ut etwas gewolbt, geftreift; bie ter Dlátterpilf. Blåtter am Strunfe breiter; Der Strunk nactit, lang, rơhrig (Wohn. unter sem

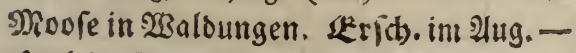

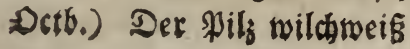
umbelliferus, Edirmpilz. 
d ber Satt ftarf genơlbt, feingeftreift; ber Strunt Duinte, lang, nadít, roobrig; Der Pil; flein, burd)aus nußbraun ( WOl)!.

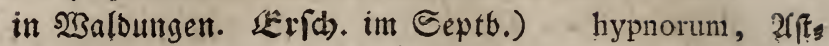

e ber ḩut fefr büne; bie Slåtter fpar. moogourtiling.

fam; Der Strunf nackt; Der \$il tlein,

I woll,

a aufrecht, zottig; bie slatter weißß; ber Şut fein zottig, balb: fugelfớrmig (Wou. Mioofe, alte Wflanienftengel. Serfd). int Suni) $\quad$ " villofulus, feirs

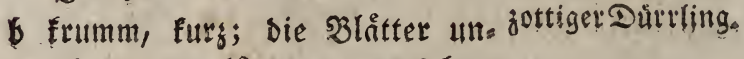
gleich; bie längern etwas bers ablaufend; Der şut vertieft; ber \$ily burchaub fapuşinb:aun (WDbn. im Sdlamme ftebender.

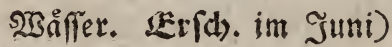

a q u a til is,

2 rơhrig, bưnte, lang; ber f̧ut ftern Sdjlammpily. fơrmig geftreift, gloctenfśrmig, im Alter flach (Molnu. unter bem Nioofe, eingeln, aber gablreich.

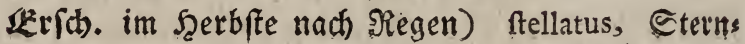

f ber f̧ut flach, tellerformig, fleifchfårbig; pily.

ber 2 sirbel aufgetrieben, ber - iand rumglid)t, und geftreift; bie Slątter meiß̈; Der Strunt fleifdfårbig, róbrig

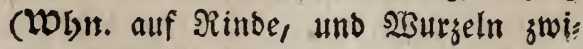
(hen bem Noofe)

parafiticus, iffmas pojender Slátter:

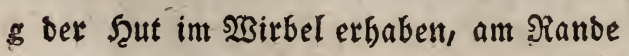
freifenlos; Der Strunf lang, bofl; pilf. ber \$ily fefr fleit, burchats weif (WObn. auf abgefallenen $\mathfrak{B l a ̊ t t e r n ~ u n s ~}$ Mgogitåminen)

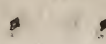

longipes, langfüF figet Slátterpily.

h oes 
h bet Scut armblatterig,

a rebr flach; bie Siatter gleichlang;

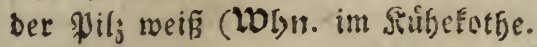
(2⿺辶斤 d). im Detb.)

paucillaminus,

6 nod) fleiner, als beym vorigen; ber Strunt febe Eurz, faum ingegen, Divtlingel zwar lotbrecht auf bie Flâche bes Suteg, aber am Rante eingefenft (Wolyi. im shoder alter Stécfe) lateralis, feiten.

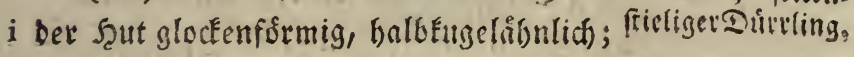
Die Slåtter fparfam, breit; der EtrunE auffteheno, ro̊krig, ant Srunde zottig; Der $\mathfrak{D i l}_{3}$ Durd)aus nelfenbraun (Wons. zwirchen Den Riken oder Sinde beg

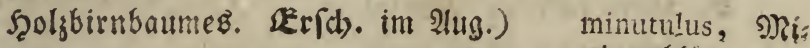

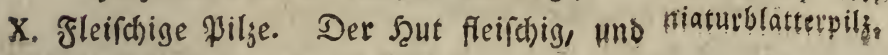
nidft's ber vorbergebenden.

A. Der f̧ut gemoilft,

\section{I glatt;}

I ber Strunf bictit, unten bicfer;

2 mit eituem falichen Ringe,

a meiË; bie Sllêtter veilenrotb; Der f̧ut roftfirbig (Whlt,

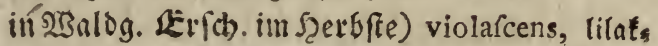

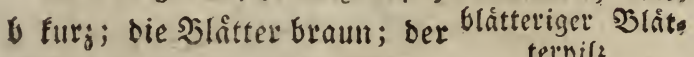

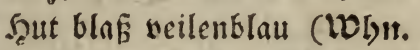
in 23 aldg. (Erido. im f̧erbfte) a me thy ft in us,

b obne Strunfring,

a glatt, zumeilen in ber Mitte amethyft fárbiget: פilättetpily.

bidfer; Der Scut grưtulicht, feucht, unb etwas flebrig; bie Slåter faneeneif (WWhn. in $\mathfrak{W a l}$ a

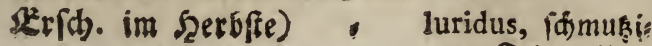


6 furs; ber f̧ut famt ben \$låt. tern veilenblau (Eerfd). int STetbfie)

coerulefcens, veis

t Der Strunf bobl,

a und wie ber Şut wadtsfárbig lenótauer Bjlåtter's fhrwefelgelb, fofmierig; bie

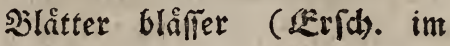
Septb.) = " = ceraceus, wactiss

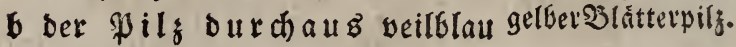

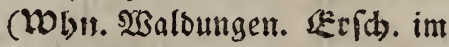
Scerbfte)

hyacinthinus,

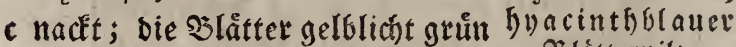
(Whit. in গabelgofgern. (Exfd. im f̧erbfte) = mutabilis, wandels

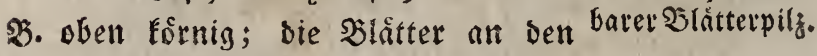
Seiten brâunlid)t gelb, ber Strunf unten sidfer; Der Silg blat - violaceus, blauer

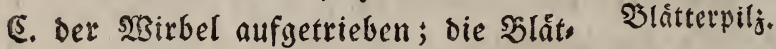
ter gezåfnelt; ber Gtrumf nalgen. fơrmig, rôhrig, nackt; Der \$ill weiß̄; flebrig (Wobn. Fichtenwålber. Lisrdb. in Detb.

- denticulatus, ges

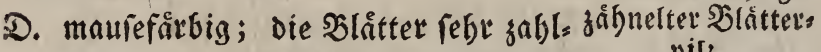
reich weî́; Dex Strunf bid)t, nackt, pily. wei $\bar{\beta}$, an Grunde bicfer (WWhn. in 23 al,

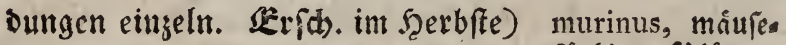
(E. Die Slâtter ro̊thlicht, braummerbend; fârbiger blattetDer Strunt bidyt mit einem Rragen. pily. refte (WDhn. auf Wreiben, WBiefen, Miftbetten. (Evid). im Suni, Juli) campeftris, Éam: F. oben zimmetfårbig, am Ranbe blås pignon. fer; bie \$lâtter gelblicjt; Der StrunE glatt, nacft, bohl; Das Fleiich wein

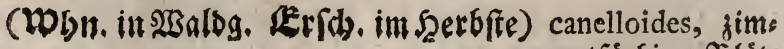
metfårbiger 'Slitt terpily.

ङ5. bie 
(3. Die Slåtter geferbt, braun; ber Strunf nackt, rồbrig, hod); Der Pils rehfirrbig, flebrig (Wonn. im Mioder

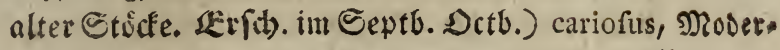

B. Der f̧ut vertieft, ober gemoltbt, ora. pilf. niengelb; bie Şlîtter bellgelb; Der Strunf rofbrig, fojuppig, gelb, mit fals (d)em Ninge, dab gleifh blafichmefels

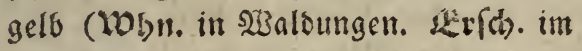
f̧erbffe)

C. Dex Şut am Rande gewólbt, braums citrinus, oraniens gel6er'Slåtterpirs. roth); die Slåtter weiß, in bie Etreife Des Etruntz Gerablaufend; der EtrunE voll, geitreift, mit falid)em gelbem Siin. ge, unten bicter (WObrr. Şaldungen. Eivis. im Şerbfte)

D. Der Şut glockenfórmig, braun, am placenta, Yfans nenÉdientáttet: Ranbe geftreift; bie Sllitter gelb; ber pily. Strunt nackt, furz, volf; meifteng zween aneinander, bef̧wegen ber f̧ut ungleid wird (Whlr. in $\mathfrak{B a l d u n g e n )}$

E. Der f̧ut mit erfabenem Ş3irbel, oben grandis, grofer Blâtterpilz. mâufefằrbig; bie Slătter alle gablig, unt wie ber lange, ringlofe, rofbrige Strunt fd)muţig tweí (Whn. gefellig,

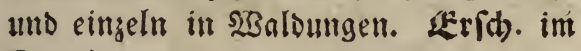
Sept.)

F. ber f̧ut flach),

mufcoides, miesers thonartiger $\mathfrak{B l}$ lits terpila.

a etwas in $23 i r b e l$ vertieft, grůn; bie Blätter ziemlid) gleichlang, gelb; Der Etrunt nacft, bicflidbt, voll (Ërid. in Serb/fe)

,

tricolor, gruำt Tiubling.

$b \mathrm{im}$ 
b im siriebel erbobeln, glatt, oranient gelb; bie Slâtter etwas herablaus fenb, idwefelgelb; bas fleifh gelb, licht tweiß̄; Der ভtrunt bicht, glatt, obne Eaamenbaut, geftreift (Wolnt. an $23 e g e n$; it $2 \mathfrak{B a l}$ loungen gebåuft. E.rdb. im Szerbfte)

G. Ser Şut gefáttigt roth, glatt, flebrig;

equeftris, भittels pily. bre Siltter gelb; ber StrunE loder, flebrig, roth, ringlog (Woltr. Sỉaloun= gell. Serfd). im setb.)

H. Der \$ils Durchaus rebfärbig; ber Strunf waljenformig, nactit, woll (Woly. it Nabelfortsern.) , , "

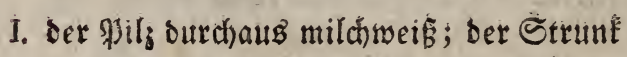

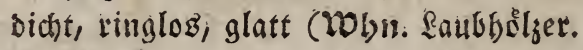
(Erid). Eeptb.)

-

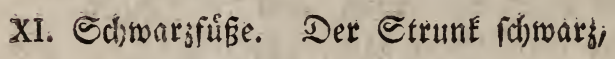

a pferbehaarfơrmig, glatt; ber şut balb fugelformig, weislidit, gefteift; bie 3lätter gleichfårbig, mentg (WObn. auff abgefallenten Fichtengapfen und Fỏbrens nabeln. 位rid). im Epatronmer uns hereffe) = " -

b hoh, nadkt, rỏbrig; ser şut siemlid) mager, gerwelbt, vertrocfuend (WDIn.

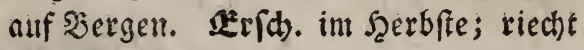
wie Sinoblauch)

xil. Eaftrublinge. Sogne EtrunE,

a vielfaltig, weißí, oben filstg; bie slåt. ter alt ber Sdineide bohlfeblig (WDitr.

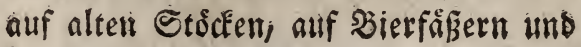
Sutchenrinben)

âlneus, Eutlett blâtterifilf: pily.

änd roface cis š 9) Zeernabelabulis

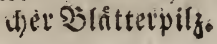

álliaceus, Snob́: Inutdbláttetpilfs

miniatus, mentigs totber Siátters

difciformis, telleits formiger $\mathfrak{B l a t t e t ' s}$ (n)

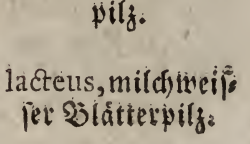


b leberartig,

a feitwairts anfitisent, bick, werfdieden fårbig, fatt Der Slatitter tiefe, laby)= riittffórnitge Gẳnge (Wh SBáumen)

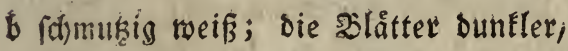
quernus, Eititens blättetpily. feitmarts in einen furzen Strun

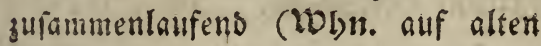
Etwifen) $\quad$ femipetiolatus,

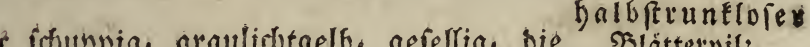

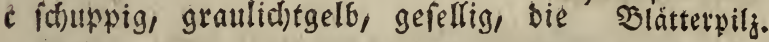
Sblåtter bunfler ungleidy; ein furjer, wagerecht eingelegter Etrink ( 20 bn. auf alten (Eid)enf(todfen)

querceti, Bictiens Frockelatterpila.

\section{Agrimonia, Doermennig.}

Die Etengelblatter gefiebert; bas ingleid)e

Slåttchen geftielt; Die Früchte fteifborftig (Woln. an Straffen, Siamen. Zlh). Juli,

\section{Agroftema, Siaben.}

Sottig; bie Relche fo lange als bie Sllume; bie blumenblatter gong, obne Intåke

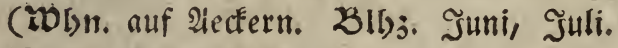
(Gebr: Medicin) *

Githago, gemeir net;

\section{Agroftis, Strauģrab:.}

i. Olne Girannen

2 bie Sifpenafte febr enge zufammengego. gen; bie ふllutthchen fritzig (WDlyn. Feld. wege. 21b). Juni)*

b bie Rifpenåfe wegftehend,

fylivatica, $23 a$ alo

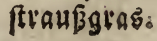

a Gaarfein; bie felchlappen oletalang 
1 Der Stengel friechend, ranfent (Whtt. Felotwege. 23!ls: Iuli) ftolonifera, aus; 2 fdhmal, fpikzig, geförbt; Der Salm laufender. aufredt (Woln. Gebuid)e, Straf= ren, פiaine. 23lly. Juli)* capillaris, baat*

6 nach bem Berblübell nieberbangend; formiges. Die Sieldtappen elfistrmig, lanjets

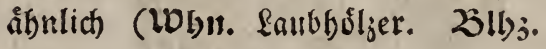
Juni) alba, weibes:

II. Nit Girannen.

a Die nutbere Spelienflappe mit einet fefr langen Siranne, Die unter Der Spize bervorfoimm ;

I bie Sirpe rebr weitfoneifig Whn. AecF. Zlls;:

2 die Siipe ver $=\{$ Juni, Juli. * engert $=\int$ Gebr. Fîrberev Spica venti, 2ffets ftrnusgras. interrupta, untets"

b bie åufere Epelzenflappe am Girunbe brodines. langbaarig, wo eine abgeglieberte Siranne betuorfơmmt, Die lănger als oas Silglein ift; die Rippe länglid)t (Whn. magerer ßoden. Zlbj. Suli. Gebr. Dedfen zu flediten) arundinacea, to 5 trs

c bie Aebrchen mit langen, geldhlångelten artiges.

Grannen; Drey Tiipenåfe aus eben Derfelben Stelle; die Slñtter borften.

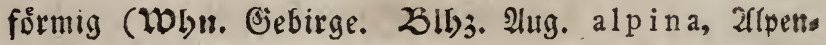
d Die Siâlglein gefâtbt, fpiţig; bie Spels frraubgrns. zen turzer als die Sålgletu; Die Giran: nen einmaits gebogen, linger als ber Sielch; die Salme am Girunde nieber.

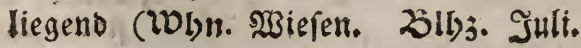
Gebr. Futter)

canina, Seunos. frraubgraร. 
Aira, Sd)mile.

I. Bramenlog; bie SRipue albeinanter ftes bent; bie Shlutthen lânger als ber Sield); Die Slätter flach (WObn. feud)te Sylefen,

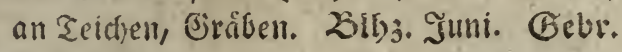
fiutter)*

II. Mit Bromnen.

aquatica, $2 B a \prod e$ :

(if)mele.

2 Die \$låtter boeftenfơrmig;

a bie SHame viel linger alE bie Epel. zen;

I ber fraln armblotterig; bie Sli then an Grunbe bebart (Woln. şerge. ¿ll)j. İtmi, Juli) montana, כerg.

2 sie Slåtterfleciben edfig; bie Slli. pitmele. then in einer ăbrenformigen ?itpe

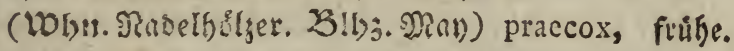
3 die Rifpe attseinanter fétend; bie Epelsenflappen an ben 'Episen

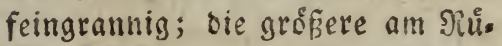
cken langgenmig (10bur. durret 2iobent. 25!53. Juli)

caryophyllea, nelo Eenaitige.

$b$ die (S)anne gegliebert, bas untere Gilipo fadenforrmig, braun, dab obere baarfơrmig, weifi, zwichen benden eilr feldfformiger Ning; bie Sirpe

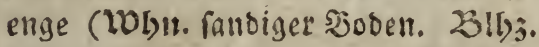
Juli. Gebr. als jumg zum Echafs futter)

- b bie Slitter fefr falmal, (trodfen) eins gerullt; bie Riffre (blutgend) aแbeinans ber ftebeno; sie Epelzen unbebaart; bie åufere Speljentappe 4jobnig, mit ciner Giranne am Grunde (Whyn. 2 Sie, [ก. 23!53. Jull. (Gebr. Futter)

canefcens, graue. 
e sie Sllather flad); Die Siffenaffe wego febento; sie Epcljen unten gottig; Die whEere Sinppe mit einer Gitanne, Die fo lange, a!s ber Seld) iff (WDIt. Wiste, fen. 23ins. Jimi. Gebr. Das Girab jum Futter, sas Etrob jul gefloditent I(rósiten)*

\section{Ajuga, Ssimfer.}

2. Siertantig puramibeniôntig; Die SIlatter ecfirs cingerounitten (Wosu. trodine, fonnige

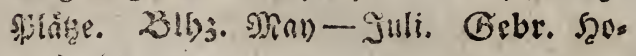
nig) * shramibenformig; sie Şlitter enforts mitg, nidis edig; sie Seldie bejaart, faft gottig (blly. Juni. Wobir. แun Surgballs (en) $=3 \quad, \quad$ genevenfis, ben

c Der Eiengel sumb, alls sem Grunbe fries

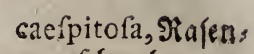
(i) mele.

pyramidalis, putras midenformige. fergúniel. d)ende Senten (Whn. STicfet, Etraflen. 25lly. frubling. Gobr. Fintberey, ben Sienen) *

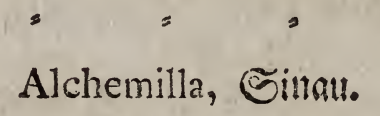

reptans, Bsilden: guinjel.

a Die Blâter lappig (TOlgn. fette $23 i e$ en. 2315;. ITan, Juni. Gebr. In:znev, Futs ter, Sobgerb, J̧onig) * " (jebirge. S15j. Juni)

\section{Alifma, Srofhloffel.}

vulgaris, Frauen mantel.

alp in a, 2flpems finau.

Die silntter enfơrmig, fpitig, nervig; bie Frudht frumpf, breyfantig. (Wobu. Grå. ben, Teide ac. Zlbj. Eommer. Gebr.

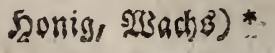




\section{Allium, \&aud).}

I. Die Dolbe bulbenlos;

A. bie 3lătter aus ber 23 urgel; ber Sdjaft blätterloz,

a faft rund; bie Srsurgelblåtter langets formig, geftielt ; bie Doldenftralen gleich goh (Wohn. Eiebirge. 23!h;. Jutni. (Eebr. Epeife) , urfinum, כadent

b von ber länge ber $\mathfrak{B l}$ li̊tter, biefe Enoblauch. rullo, fpiz̧ig auslatfend; Die Trå ger einfad), die Sulbe vielzấning (Woln. Felien, Giărten. 2blb3. Niay-Eeptb. Gebr. Speife) Schoenoprafum,

c ăı bem zwebfdneibigen vierfan. Ed)nittlauds.

tig; bie Blătter breitlicht, rinuens fơrmig; bie Trioger einfach) (Whln. Felien. 25!b3. Juni) angulofum, ectigs ftengliger.

D am untern Enbe Gaudhig; bie \$llats

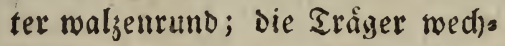

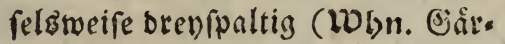
ten. SIlbj. Juli. Gebr. Epeife,

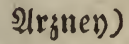

Cepa, ลิwiebel.

e zwevfdrneibig; bie şlåtter linien. formig, auf ber stůffeite runt. licht; bie Iråger einfach); bie

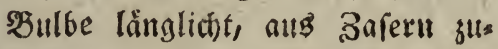
fammengefesct, im alter feitwierts gerid)tet (Wobn. in Şecten. 251 b). Juli)

B. Die Blitter am Sdafte,

fenefcens, গ̧erg: Enoblaudy.

a flach; bie $\mathfrak{b u l b e}$ mit einem Şurgel netge bedectit, lănglicht

I bie Tråger brempipaltig (Molhtt. (Bi̊rten. Zlb). Juni, Juli.

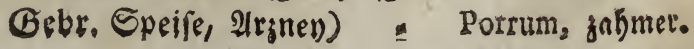


2 Die Sriger einfady, linger als bie Slume; sie S)olde fugels formig; bie \$latter erliptifd)

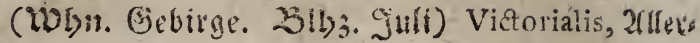

b waljenrund; Die Srager medfeleweife manishatnifos.

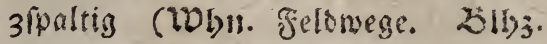
Julli)

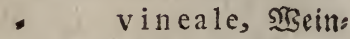

II. Die Dolve Gulbentragens; Die Dlatter bergs(aนt).

ain Edjafte.

a f(ocb),

a fein fågezånnig, segenfirmig; bie stäget wedhelsweife shaltig (Tolyn. binter Bounen)

arenarium, Ennos

6 an Reffen nergig; Die Dolde zwen: Enoblaud.

fuleibig, bie eine Echeibe febr lang;

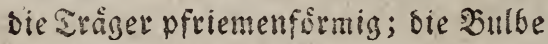
einfuch (Mobu. fette șiejen. 23igj. Şuli, 214.)

s

carinatum, Fiels blatteriget.

b Galbruno, roficig, gefurct); stuo Dolben,

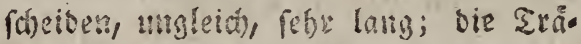

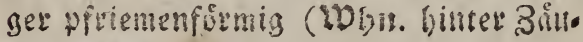
และ. Bibj. Juli)

\section{Alopecurus, Sudfsidfuans.}

oleraceum, Bials tenlaulif).

Die Bhtumenfelgen granmenlos.

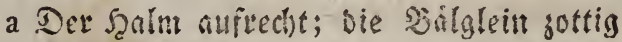

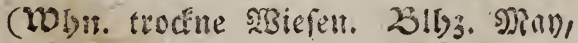
Juni. Gebr. Gutter)* = = pratenfis, 2ßiefents

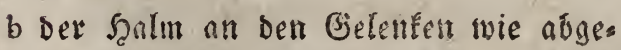
fuchsfolwan?.

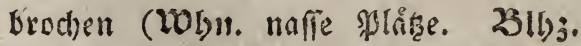

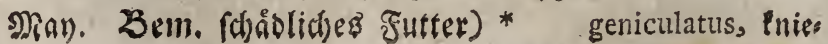
formiget. Alfine, 


\section{Alfine, Scifinneroarm.}

Die Slumenblätter 2tfeilig; sie Slâtter bers: nifnlid), enformin (MBgri. allentbalben. 23ihj. Jruibling mo Enmmer. Gebr. 2ogelfutter)*

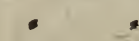

media, gemeinet.

\section{Alyffum, Steinf̂raut.}

A. Sirautartig; ber Etengal aufued)t; bie Blitter lanjetformig, volfommen gang, uno nevít bem Etengel und ben Edjôtt. d)en granfilgig; die Cabotthen langlidst,

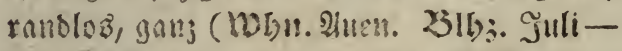
Scto. Gibr. cill-guteb Ecljaffutter) * incanum, graues.

B. JanlGfraltartig; bie Slatter mit geftirn. tcn STunten befert; feche beuteltragende,

a uno 4 beutcllofe Etaubfioen; bet Etchuel weitfoincifing (IObn. Şeis Den. 2lb3. Mian, Jumi) ${ }^{*}=$ calycinum, ges

b unto 6 beuteliofe Etaubáten; Die feldutes. Etengel aufred)t, fiemlid einfad); bie Selche bieibend (IDlyu. Iecter. 23lb3. Juni, Juli) " "
Amaranthus, Imarantf.

femidodecandrum, balgzwólfmánni: gez.

a Die Blitter an Der Epitze ausigeranbet; Der Etengel weitformeifig, etwas nicber. liegent; sie Achrdhen aus ben Eeiten,

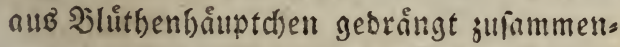

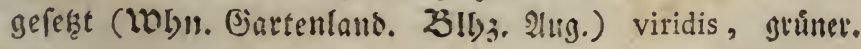

b bie Şlâtter gang; Der Etengel alfrecht;

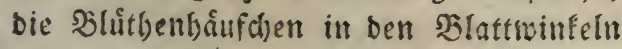
unb an ber Epitze weitlơufig (MLn. 2lecter, (G)ห้rten)
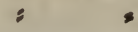

$s$
Bl i t u m, Sllut: Evalt. 


\section{Amygdalus, Mandelbaum.}

Die Slåtter grunomårts fómal zufammen. laufeno (Vobnt. am Donnufer. Gebr. Branbtwein, Sonig)
Anagallis, (Saudjheil.

Die ßläter unfaffend, enformig; ber Stell. gel nieberliegend;

a bie Şlumenblåtter gefrangt, etwas ge. ferbt (Wolu: Aecfer. Blb;. JuniEept. (Gebr. Arzmey) $=$ phoenicea, rother.

b bie Blumenblåtter fpißzig geferbt, etwaz gefrangt (Blb3. Jutni)

nana, 3 welge manoelbaum.

\section{Anchufa, Ddfenjunge.}

a Die $\mathscr{A}$ bren einfeitig, eingerollt; bie $\mathfrak{B l u}$ s then bachstegelforrmig; Die \$latter langet: âfnlid) (Whn. Felowege ůberall. 23!b;. Man, Juni. Gebr. Stonig, Epeife, Făr: (berev)

b bie Sluethenåfte 2theilig; bie Sllitter lång; lid)t gezogen (bll);. u. Gebr, wie oben) Andromeda, Antromeoa.

officinalis, gea meine.

a ngu ft ifolia, id)male.

Die Slutthenftiele gehånft; bie \$ltumen en.

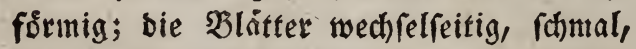
am Ranbe zuruefgerollt (Wobn. Morifte. 25(b). Juni)

\section{Andropogon, Şartgras.}

polifolia, poteys blâtteriger,

Die Aebren gefingert, linienformig, zottigs bebaart; bie Slutbdien ftiellob, gepaart, cin gegranntes, und ein grannenlofes

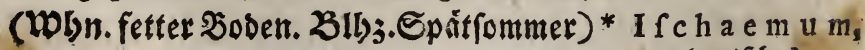
Deut $\{$ thes. 


\section{Androface, snammşarnif}

a Die sisurgelblatter, po wie Sdjoft uns Selch berhaart, gehâuft evformig; ber Seldh treufelformig (Wobn. bey beysen madfte Gebirge. 2Jlbj. Juli) ="

$b$ bie Bratter lattg, fhmal, slatt; bie Doloe

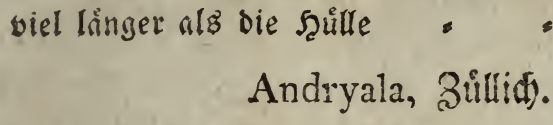

Die Silåter långlicht eyformtig, gatly, mit bervorfpringenden Beftren, glatt; ber Etengel einblitbig (Wolnn. Mlipen. 23ltyj. Suli)

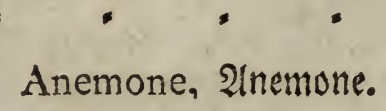

Chamae jarme, Eergmannbhat: niiff.

1 a cte a, militgo weifit.

uniffora, einbla thiget.

1. Die Caamen gefthwingt; Der Etengel Iblutbig.

A. Die Stengelblåtter umichlagfơnmig;

a bie reife Sllitbe ůberbangend; bie Slátter boppelt gefiebert,

a bie Etudfe linienformig; bie Şlumenblitter gernde; die ふ̉lu: the bebaart (SII); Naßy) Pulfatilla, nrobe

b die Stuicfe an ber Epite ges Suthenidielle. jơntit; bie Slumenblätter an Der Epiţe zurúd gebogen

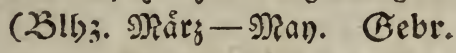
গ̧onig, গ্ৰad)8, Fårberey, Ipo: thefe)

b bie 23 urgelblätter gefinget ; bie pratenfis, Kleine Sititemidelle. Slittchen zeridhitten (LDbu. Aluen. 23lbj. 2uril)

c bie slåtter einfad) gefiebert (พDLn. patens, offene Sidjenidelle.

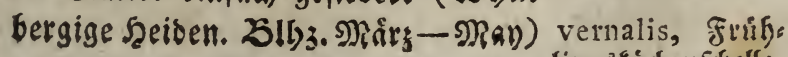
lingétúthenforelle. 
B. Die Stengelblatter 3 fach, zufammenge. wad) ren, 3mal zufammengeferat, vielfad) serifhnitten; bie Samen raub (Woln. Gebirge. 23ly. Junti)

alpina, 2llpenanes mone.

II. Die Saamen ungerdhwangth

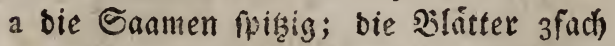

a brenfingerig, zeridnitten; ber Eten.

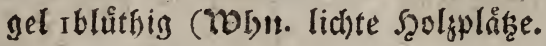
2315j. Nints-Niay)

nemorofa, $\mathfrak{B a l b s}$

$\checkmark$ orentheilig, eingeldnitten; bie \$lt. anemone. menblatter foft tellerformig; ber Stengel meiftens 2bluthig (HOl)ir. 23lb. wie oben)* . ranunculoides, b bie Saamen enfiermig, madt, nieberge. selbes gralobifn: bruflt; bie Sllithen in Dolben (Wolnt.

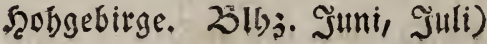

\section{Angelica, 2ingelife.} (d)en.

narciffiflora, mate ciffentiúthigezfne mone.

a Die Blätthen einander gleidh, elfformig,

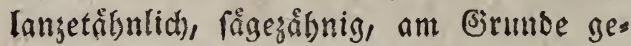
nau begranjt (Wobn. feltute \$lâţe, ufer. 23!b3. Jull, 2ug. Gebr. ben Sienen, fur bie Sctweine) * " sylveftris, wilbe.

b Das ungleiche, ober oberfte ふlättdien lap. pig; alle an ifren Etielchen etwas bers ablaufend (Wolsu. und 23lbj. wie oben. Gebr. Sranbtwein) *

\section{Anthemis, 2nthemis.}

Archangelica, eole.

2. Der Blưtheboben Eegelfơrmig; Die Blątter glatt, Doppelt gefiedert; bie leketen Slatts ftucke langetformig;

a bie 2lefte aus ber gangen fänge Des Ctammes; sie Caamen mit einent Sanbe getront, vierfantig glatt (W0bu. 2lecfer. 23lb3. Șuni-2/ug.)* arvenfis, wilbe: 
b bie Affe aus Dem ofern Theile bes Etammes; die Earmen fronenlog,

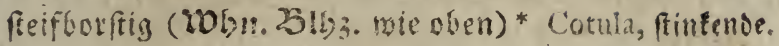

b ber Stral mit Dem Sillitbenteller gleid): färbig, gelb; sie utuen slatter boptelt, bie obern einfad gefiesert; sie Slattd)en

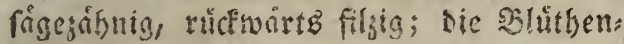
fitele als jortfenungen ber. Alefe weit ber. ab nacit (Mbn. Kilbj. wie oben. Gebr. Firferey) *

,

t inct oria, fäts benie.

\section{Anthericum, Epimnenfraut.}

a Der Edjaft affig; bie Blumen Fiad); ber

- Etempel gerade*

ramofum, îftiges.

b Der Edbaft cinfad); Die Blumen flad) wegs ftebens; Der Etempel gebogen (Wobn. beibe all Sergen. 23lbj. Juni - Ilug.) * Liliago, aftofes.

Anthoceros, Sjomeriforf.

Das SIntt am Bobent angebrideft, nusges fd) weift (robn. Ilecter. frutblbar int Detb.)

: . " lacvis, glatter.

Anthoxanthum, Siud)gras.

Die Aehre enformig, linglidyt; die Shlutgen

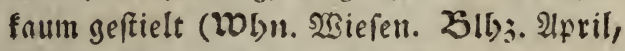
Nay. Gebr. Futter, Den Sienew) * odoratum,gelbes.

\section{Anthyllis, SSunbFraut.}

Sirautartig; bie NBurzelblitter enformig; vie Etengelblätter gefiedert, Die Blittchen un. gleid); bas Bluttienfiontchen aus zmeven

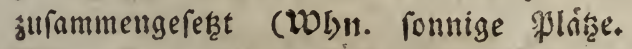
23153. NRav. Juni. Gebr. Futter) *

vulneraria, gemeines. 


\section{Antirrhinum, foivenmaul.}

I. Die Blumen gefpornt;

A. Die Slátter wech)lelfeitig;

a ber Stengel nieberliegent

I bebaart; bie Slítter eyfórmig (2Dbu. Hecfer. 23lbs. Epåtrons mer) = " " " fpurium, eyblitts

2 bie Blatter bersforrmig, ftumwf, terigeb. 5 lappig (Woln. Felien, alte Mau= erir. Zlls:. Sonmer - Cymbalaria, 3yms

b ber Stengel aufred)t; bie Aebren an belfraut.

Enven ftiellog; bic Bluthen bad). siegelformig; der Eporn lang; bie Slltter lanjetifntid) linienformig (WDlu. Hef́er, Aenger. Bll)3. JuniEeptb. Gebr. ßienen)*

Lin a ria, Seits.

c ber Stengel meitfaheifia, lebr åfig; Elaut. Der Sporn fury, fumpf; Die Sláts ter lanjetfirmig, ftumpf (IOLn. Etraf

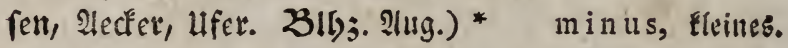

B. bie blatter gegenu์ber, linienfórmig, vierfach; Der Etengel weitffineifig; Die Sluthen trutbenformig; Der Eporn gerabe (Tolut. Strand und Ralchboden. 23l5;. Juli- Now.) *

alpinum, 2flyets lơvenmaul.

II. Die Slumen fpornlog;

a bie Bhithen in iffenförmigen Traus ben; bie Reldffticfe grofi, gerunbet (WDhtr. Felfen, aud anbere Sierge. 23lbj. Juni - 2(ug.)

maius, grofes.

I bie Sluthen in ben Slattwinfeln; bie Sield) ftúcke pfriemenfórnig, länger als bie ßlumen (WDn. bergige Biegenden.

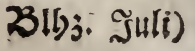

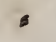

Orontium, Dos tant.

Aphanes, 
Aphanes, Grauenmintelden.

(Wobw tbonige Mecker. B!b3. Nay, Juni)* arvenfis, gemeio Apium, Eppid). nes.

a Die semselbläter gefiedert, geridnitten, gegoignt, Die am Etengel linienfórmig (10hn. Gårten. Gebr. Epcife) s Petrofelinum, Dei

b bie Etengelblatter feulformig; sie unter: terfilge. fien 5 fitigerig gefiedert (Mblr. u. Gebr. bie in Cumpfen molnnende Eorte (ctioblid), bie in (jarten zur Epeife)

\section{Aquilegia, 2lgley.}

graveolens, 3ek (iti).

Die Epornen frumm gebogen (Vobn. Sies bege, Biebirge. Blbj. Juni, Juli. Bebr. Эienen) *

\section{Arbutus, $\mathfrak{B a ̊ c e n t r a u b e . ~}$}

vilgaris, gemeis ner.

Der Etengel nieberliegent, Die Silatter wolls fonmen gang, unten pumctenlog (WObn. bergige Siegenden. B!b3. Mian, Juni. Gebr. Tabaf, Fincerey) , : Uva urfi, gei Arctium, Silctte. meille.

Die Blåtter hergfơrmig, unberwebrt, geftielt

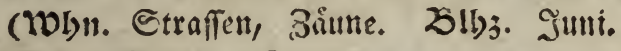
Gebr. Nebicin, Epeife) * , " Lappa, grofe,

\section{Arenaria, Candfraut.}

I. Die Slätter gegemu์ber,

a eyformig,

a ppitigig, merbig

I fursftielig, glatt (Wobn. Sautboels zex, Şecten. 23lbj. Juni, Juli) * trinervia, stelys ribgiges.

2 firele 
2 ftiellos; bie Silumen furger als Der Seldh; Die Pfange fein feifs

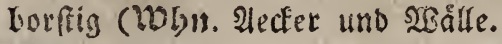
21!);. SMan)*

ferpyll ifolia,

6 leinglidst, faft geffielet, gefronst; bie

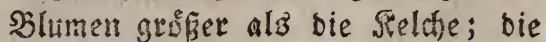
ges.

Selchblátter faft znervig (WOBn. Sie: birg. Sibj. Itug. Sept.) = ciliata,gefrangtes.

b linienformig, aufrecht, angebridét, mit zwo Futhen atif bent siuctern, am Grunde gebrångter; bie Seldblatter Iånglid)t, geftreift (Moln. bergige Bes gent. 23li)j. Juni-Eept.) = ftriata, geftreiftes.

c pfriemenformig, glatt, ant Birunde ver. bunben;

a Die Slumen linger als ber Reld); Die Sluttgen gerifpet; die Sapfeln aufred)t (20In. Stalffelfer. 23ll);. Sommer) ". . faxatilis, gelfen:

b Die Shlumen fưrger als ber Reld); Tandérant. Die Stengel aufred)t, 1 blutthig (IDbn. Etraffen. 23!ly3. IRay) tenuifolia, fdimals d fabenforrmig; Die Blattanforge båutig, blatteriges. langetformig (WDhus. abgetriebene 5ols: plåze. Zllbj. Sommer) = - rubra, rothes.

e borftenforrmig; Der Etengel gerabe, aufs recht; bie Slüthenrifpe Eundelfirmig; die Sllumenblåtter febr furs (WOJn. ? fafciculata, bums

II. Die Slifter balbruno, fleirhigt, ftumpf; Delformiges. Die Slumenblätter langetfơrmig, ungefibe stwo Blutthen auf einem getbeilten Blưthen. fitele num Ende bes Stengels : bavarica, baytio (ด) 


\section{Ariftolochia, Sfterlugey.}

Die Silátter herfförmig; bie Slluthen in ben

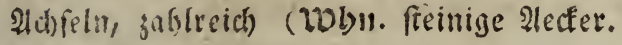

\section{5;. I):(n), Juni) \\ Arnica, srgolverley.}

Clematitis, ges meine.

a Die Britter gegenuiber, ebfúrmig, voltents men gan, (Whn. bergige Ëegenten. 23!b;. Juli-Eeptb.) , " " montana, weges

b ber Edraft nact, sam ófelog, einblutbig ridgläteriget.

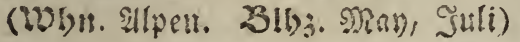

\section{Arabis, 2trabis.}

Bellidiaftrum, manß̧icbenartio get.

a Die Slatter umfaffend, faft Gersfismig,

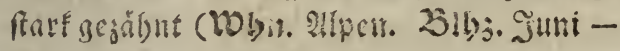
2ing.)

alpina, 2llpenatra

b Die sisurjelblåtter geftielf, enformig, lan bis. jetrifnlidí, ftumpf, gall; (oocr parfam uno (eid)t cingeichnitten); die Etengelblätter länglicht, ungeftielt (LDYn. magere Bininde. 25lb)3. Ispril, Jumi, nuch mieter im Scerbfre) thaliana, thatias

c Die untern SBlåtter leyerformig, bie obern nifise. lanjetformig, eingefdnitten, unb gans (Hobr. Bebirge. Z3lbj. Jumi-Eeptb.) H alleri, bals Artemifia, నenfuf̧. levifate.

a Die Slatter vielfach zerf(d)utten;

I oben glatt, unten filsig; bie Blutthen etwas uberbangend, in zufammengefers. ten Trauben (B!I). 2lug.) - pontica, pontis

2 bie Etudfe linienformig; Die Stengel (id)et. nieberliegend; bie 2lefte rutbenfơrnig (mbin. an Straffen. 23!l)j. Ang. Spptb.)*

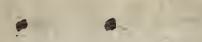

campeftris, milo ofer.

b bie 
b bie ¿latter sefiedent jerfönitten, unten filjig; sie Etudfe abermal gerfdutten; bie Sllutben in einet åbrenfornigen Sratbe, aufted)t (Moln. an Bămen, Sitinen, Sie= buffben. Jlbj. wie oben) ${ }^{*}=$, vulgaris, gemei. net.

\section{Arum, 2lion.}

Die Blatte fponsonformig, volffommen gan; oer Slatbenkolben twalgenformig (20lsn. naffe fette Oruinde. Zibs. Sgay. Gebr.

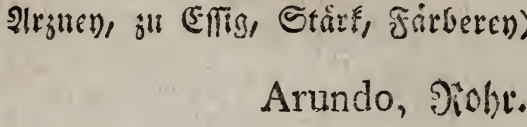

a Die Rifpe aubeinuttoer febeno; die Relche

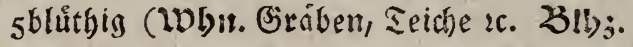
Suli. Gobr. zu Baft, Bimmerdeden, fár. beren) *

b bie SRifpe aufredt, unterbrod)en verengert; maculatum, ges meinez. bie Bälglein Iblutbig; Die Epelgen unt

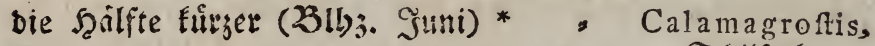

c bie Sieldhe Ibluthig; bie silumenblåtter Phragmitis, ges meines. flach; Die Slattf(heiben mit einem mebrs

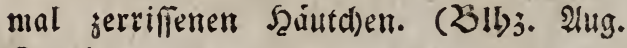
Eeptb. $=$ - epigeios, rano, Afarum, Şafelwurs.

Die SBlåter nierenformig, ftumpf (WDH. unter Sieftruchen auf fettem \$ober. 3lisj. April- Jưli)*

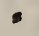

\section{Afcidium, ङd)laudpils.}

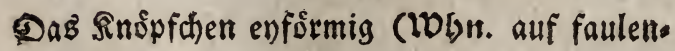

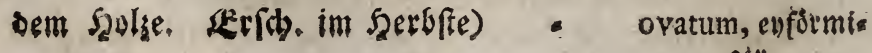
get. 


\section{Afclepias, Sdmalbenwur:}

Die Blöter enfórnig, ougchpist; Die Eten. gel aufvecht; bie Sluttben in Shufcheln (20hn. fondiger Soden, 2Galdungen. Slbj. 3au)-2tug. Gebr. Jutter fü Ziegen, fratt fladsz verarbeitet) * "
Afparagus, Epargel.

Vincetoxicum, gemeine.

Der Etengel frautartig, aufrect) ; sie SIlits ter borftenformig; unter jebem Slútien. fiele oren Slattanfäze; einer groffer

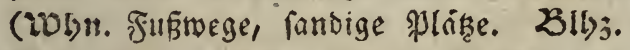
Nay, Juni. Gebr. Speife, 2ranty, ben \$itenen$$
\text { , }
$$$$
\text { Afperugo, Eđarffraut. }
$$

officinalis, gemeis ner.

Die Etengel niederliegend; bie Fruchtfeldhe zufanmengebruㄷt (Whnt. Steingaufen und fetter Boden. Zlibj. Nian. Gebr. Epeife, Futter) *

procumbens, nies Derliegender.

\section{Afperula, rsalomeiffer.}

a Die Blátter ad)tfacis lanjetformig; die Blutbenbưnbel gefticlt (Wobn. in Raubhéls 3ern. ઊlbj. Jull, 2ug. Gebr. Den \$ier nen, Futter, wider Notten, Argney) odorata, wabres.

b bie slatter linienformig, bie untern fed)s: fad); bie mittlern vierfach (WWn. WBie: fen, Sceiben. Zlbj. Juni, Juli. Gebr. farirberen)* . . tinctoria, fâtben

c bie Slätter vierfach) . Iinienforrmig; Die Det. obern હegenblifter mit SBlattanfingen; Die slumen vierfpaltig (IDl)n. magere Grưn. De, Felien, Mahern, Eebr. Färbered) * cyn a n chica,

23) หåunerourzel.

a bie 
d bie Blatter 6-8fad); bie Blitbenbundel an ben Enben friellos (MObn. fteinige Aedfer. Żlb3. Juni)

\section{Afphodelus, Iffooil.}

arvenfis, Ffoetr meyetínut.

Die shitteiribbe ber Dfumentricke tiefer ges fâtbrt; Det Stengel nact; die Blitter Degenformig, fielábnlich) (WDhl. bergige (jegenben. Bibj. Juli) $\quad \ldots$ ramofus, diftiget.
Afplenium,
Streifenfarm.

a Das Blatt volfenmen gans, gleichbreit langetformig, am Etwme nugigefintten; Der Etrut zottig (20hus. Selien)

S colopendrium,

b bas Slatt jefiedert, bie Blittchen faft feilifisunge. tellerfirmib, geterbt, won einander ents fernt (WOhn. Jelfenciscn, nlte Siantur) Trichomanes,

c bas blatt aftis gefiedert; bie Ilefte wed). 23 isertbon. felsmeife; bie Bláttdhen feilformig, geterbt (20l)n. wie oben)

\section{After, Eternblume.}

Ruta muraria, Minuertaute.

a Die Blätter frumpf, borftig, gans; bie Selchichupen gefrangt;

a ber Etengel einfach, einblutthig; sie Slätter verféturt lamzetformig (WObn. (s)ebirge. Zlisj. Juli, 2lug.)

alpinus, 2f(pent,

$\checkmark$ Der Stengel mebrblitbig; bie Sield). fremblume. fd) upwen ftumipf; Die Blatter langet. förmig, brevnervig (Woln. F⿻ůgeln, Derge. Zlbs. 2lug. - Octb.) * Amellus, blaue.

b bie Blitter enformig, edfig, gezánt, ge. ftielt; Die Blútben all ben (Enden; bie Seld). f(h)uppen blattífnlich, megftebend (Wolgn.

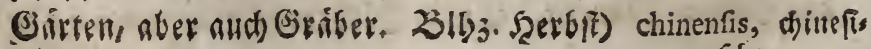
rige. 


\section{Aftragalus, ₹ragant.}

a Der Etengel aufrecht, gerabe, glatt; bie Siátter farf geficoert; bie sluthen in Trauten bangent; Die Scullen faft brey. fantig, an beyden (Enoen ipişig (MOlyn. Serge. 23ly3. Juni, Juli) -galegiformis,

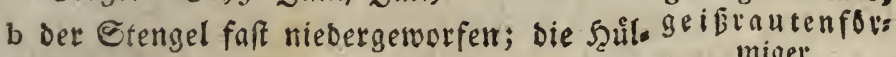
fen faft Fugelformig, nufgetrieben, zuge. miget. fpist, behant (Whn. Wiefen. Wlbj. wie oberr) * $\quad$ : " Cicer, wilbe Sis

c Der Etengel nieberliegend; bie ઝlutben in Irauben;

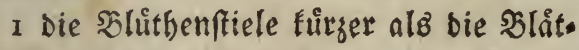
ter; Die Scurlfen faft orenfantig; bie Dlăttchen eyformig (LDLm. an Etraf. fen, 2lecter. 23lb3. wie oben)

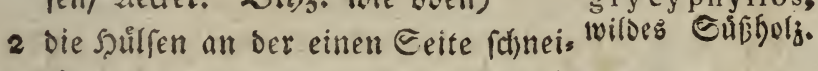
Dig, an ber entgegengefersten mit eis ner tiefen fångefterbe (Wohn. Gie. birge) . . . " alpinus, 2lipen.

\section{Aftrantia, Iftrans.} tiagant.

Die SIletter 5-7theilig; Die Ibeile feid)t 3ipaltio, am Rande Doppelt fágegribnig

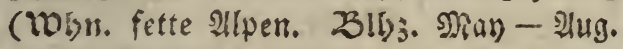
Gebr. $2\left(r_{j} m e n\right)^{*} .$. major, grobe.

\section{Athamanta, Şinfiatulus.}

a Die Fructit zottig;

a bie Sglátter boppelt gefiedert; bie leke. ten Sblättchen gefiebert, eingeidunitten; Ser Etengel ectig,

I an ben Gelenfen feingaarig; bie umichlagblattchen furger alz iste

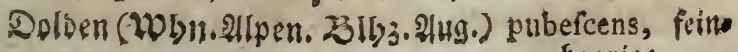
ban:ige. 


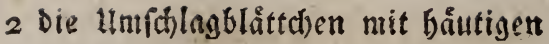
Painbern; bie fonderbeitlid)en lån. ger alB Die Dolodxen (WDLn. ŞOb.

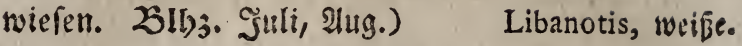

6 Die 3 lätter orenmal zufammengeferst, bes baart; bie Slumenblatter tief bersfers mig; Die Slattfengel meiffens 3 blätte. rig (Mobn. bobe (jegenden) s cretenfis, cretifaje.

b bie Saamen nact'; bie Solâtter boppelt gefiebert; bie Slăttchen alif Die Mittelribbe lothrecht, in Der zwoten Dromung feilfors. mig, eingeidnitten; Die Studfe an ifrem

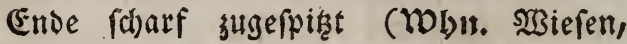
Felfen) $\quad=$ ", cervaria, (d) woarge.

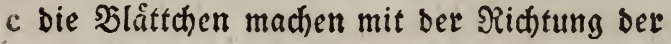
Nittelribbe redte unb fumpfe Sinfel; bie Spiz̧en ber lek̨ten Blåttchen fnorplig, weiß (WObn. bobe WBiefen)

Oreofelinum, כergpetellein.

Atragene, Doppelblume.

Die Slatter boppelt brenfingerig; bie Relch: blätter blumenblattíbnlich) (WWhn. Scobges birge. 23lbj. Juนni)

al pina, 2llpert. opppelblume.

\section{Atriplex, Meloe.}

a 2Ule รllitter beltafơrmig, fponbonåhnlich; bie fieldhtbeile Der meibl. Blutben belta. formig, meblig (Woln. an Băunen. 25ll)j. Spátfommer)

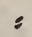

-

b ber Etengel weitfhneifig, famt ben 2leften faft nieberliegend, frautartig; bie unter. ften Slátter pfeilfơrmig, bie obern láng. lid)t eyforrmig, bie oberften lanzetfórmig

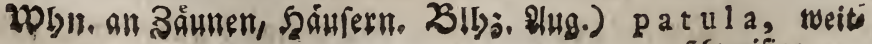
isjueifige. haftata, fponooni blátterige. 


\section{Atropa, 穴llfirfae.}

Der Stengel frautartig, bie Şlåtter eyfo̊:. mig, gans (Whin. in Sisaldungen, an Strafs fen. 23lly3. Juni-2lug. Gebr. fur bie Naler, Elrzmen) *

\section{Avena, Şaber.}

Bellad onna, ges meine.

A. Die Seldhe sweyblưthig; nur ein Sluttb: d)en gegrannet;

a besbe frudtear, ber Saamen ichrary. braun, อீfters aber blägelb, glatt

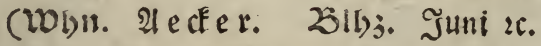
Gebr. Jutter, Epeife) * " fativa, gemeinet.

b mur eines frudt)tbar; bas mainnliche langgegrannet, bas 3witterblúthdhen

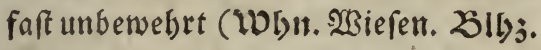
Gebr. wie oben * . " elatior, bofer.

B. Die Relche 2-56luthig; alle Blutthan mit Brannen; bie Aebratenfpuble bebart; bie Aebrchen in einer Rifpe

a bie åufere Epelzenflappe an ber Epi:

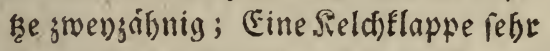
fur\} (10hn. 23iefen. 315). JuniFlitg. Gebr. Futter) * " " flavefcens, Codor.

b bie äunere Epelgentlappe an ber baber.

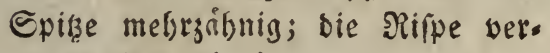
engert (mie oben) " = pubefcens, zarts

C. Die Relche 3 blüthig; Die Sllutbdhen alle behaart, gegrannet, in einer sipe (WDbr. 2lecfer. Żl5j. Gebr. wie oben)*

D. Die Sielche unb Alebrchen 5 blutthig; sie f a tua, Taubs Alebrchenftiele furs; alle Blutbd)en gegrans net, in einer Sifpe; bie Aegrchenfpuble

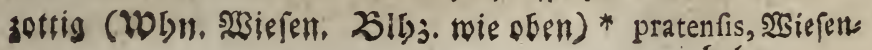
baber.

B. Bal- 


\section{B. \\ Baillota, Ballote.}

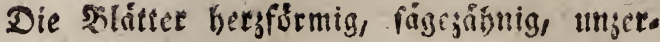

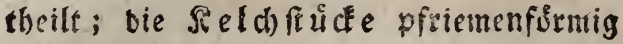

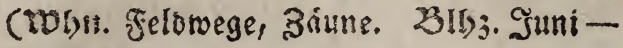
2lug.)*

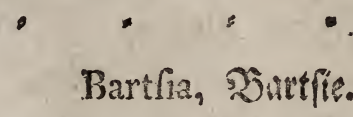

\section{sigra, fáfwarges 3indortn.}

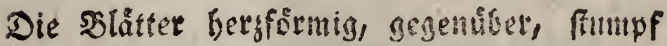

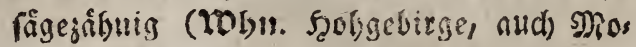

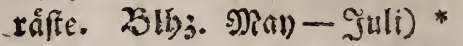

Bellis, Maáplieben.

al p ina, afpers bartfie.

Der Schaft nacte (MDbrr. an Straffen, auf

Weiben, im Barterlande. 3lb3. Das ganze Jabr. (Bebr. Irznet) * ${ }^{*}$ perennis,gemeine:

Berberis, 23 cinfifirling.

Die Blutfen in Irauben (Wobn. Şecien, Gebtiche. 23̈ly. Juni. Cebr. Epeife,

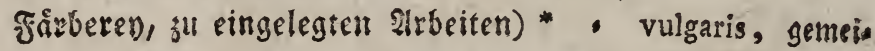
ner.

Beta, Siannen, rotise Siliben, Mangolo.

Det Stengel aufrect); die Soluthen gebåuft;

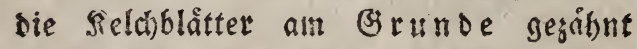
(Whit. wirb blos gebaut. 23lbj. Juni. Gebr. Speife) *

Betonica, SBetonie.

vulg a riss, gei meine.

Die Alefe unterbrod)en, Der mittlere Sap. pen ber unterlippe ausigerandet, geferbe

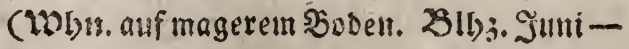
2lug. Gebr. Futter, Soltig, 2rs̆tte) *

offic in a 1 i s biaune. 


\section{Betula, 23irte, Erle.}

a Die Släter berjformig, iugeffist, bop.

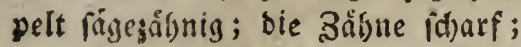

a bie Nefte gerabe (10hn. in 20rbol.

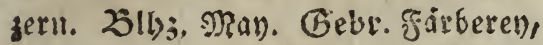
Berberen, gute Sioblen, guten গiuf,

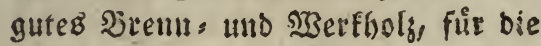

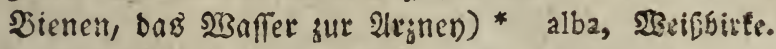

b bie 2lefte ůberbangent (1051t. 23!5j. Gebr. wie oben) * " pendula, f̧angels

b bie 3llitter eyfơrmig, boppelt fägezåt). bitfe. nig; bie Zåthne đharf; bie Zweige ectig; Die meibl. Råtzchenfticle åftig (Woln. Be. birge, in Saiern?)

ovata, evformige. c bie Shtnitter benderfeitş glatt, einfadh, fåge: zod fitele cinfad); bie Edfuppen rumjellos (WOLn. Niorafi)

d bie Sllitter faft tellerformig, utrorbentlich humilis, शrocafts frigejálug; bie Slattftiele mit Drúfen

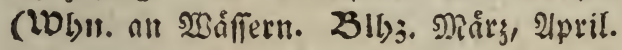

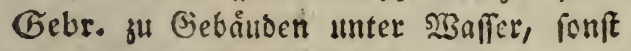
wie beu ber ŞSeifbirfe) * " "Alnus, Erlc.

\section{Bidens, 3mey;afin.}

2 Die Slatter 3theilig; Die Reld)e etwas blâtterig; bie Shluthen aufrech)t (WDbr. an Naffergråben. Zlh3. 2ug. Sept. Gbebr. Firberely)

b_ Die Slåtter lanjetrórmig

tripartita, 3theis liget,

a ftiellos; Sllitben uno Saamen auf:

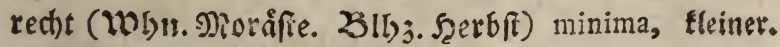

b umfaffent; nur bie Saanen aufred)t; bie \$lutben Hberbangend (WDbn. 23!bj. (Gebr, wie oben)

cernua, Runigun: Dentiaut. 


\section{Bifcutella, Doppelífillo.}

Die Blúthen fpornlos; bie Schottcher einem \$aare am Sanbe verbundenen $\mathfrak{E} e l l e r$ åfn, Iich, glatt (Wohn. Giebirge. Z Blly. Suni, Suli)

\section{Blafia, S̈lafie.}

Didyma, semeis

net.

Nian fennt nut eine 2 rt (Woln. auf thoni. gen Aecfern und tiberid)wemmen (j)egen: Dert. Kerfb. mit ber Frudht im Scerbfte) pufilla, Eleinfte:

\section{Blitum, Schminfbecte.}

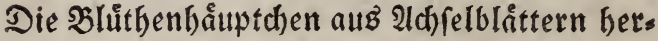
vorfommeno (WOhn. an alten Maltern. 23lb3. Sonmer. Gebr. zur (d)minfe) * virgatum, wuthens Boletus, £i̊cherforwamm. fortmige.

I. Shne Strunf, ober boch mit fehr fursem

A. Şolzig, ober faft bolzig;

a gepolfert,

I fefre feft; qutweilent ift eit Strunk Da, fteht eintwartg, ift braun; ber Sd)wamm unten flach, åtrferfit feinlơd)erig (WWhn. allf Bånmen. Gebr. zum 3unber)* "

2 woblriechend, auf benden Seiten igniarius, 3unset:

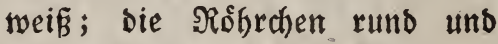
ectig, refre beutlich) (BDlun. auf

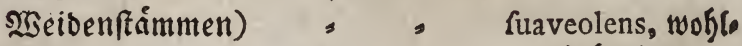

3 leberartig, lappig, bachsiegelartig, riechendet. gebáuft, oben fammetartig, ges wo̊lbt; unten feinlooderig, flach

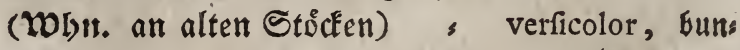

b viertelfugelformig, weiß, unten fómus [千 boamm. sig weiß̄ (WDhn. an Baiumen)* ter.

$21 \mathrm{~b}$ us, weiket: Daflótbetpily. 
e bufågnlich, braun, oben mit farbigen

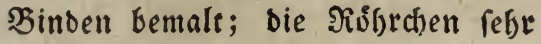
flein, unrcin wein (WObn. auf alten Saumftámmen)

,

hippocrepis, buf:

d in Glitterige \&appen getbeilt; sie fórmiget.

Rappen bad)ziegelforermig, Erats; bie Ridgrchen verbunben; bie Nündun. gen fefre flein (Whlt. an alten (5i, (d)en. Bebr. efsar)

frondofis, endis

e ber Scut vertieft, soendeltreppenfók, vienformiger. mig um eine Epindel berumlaufend fcalaris, stsendets

B. Breyartig, nur mit Einer Sberflid)e. treppenlóderpils.

a Eine brevartige, meißse Srufte, mit fchiefen malgenfớrmigen sidgren auf ber Sberfliche; die gange unterfeite auffistseno, nach bem Sertrodinen fils sig (WObn. an faulen Stócten)

Medulla panis,

b weiß̄, ober wafferfärbig, gans alts Biodérummenat: tiget.

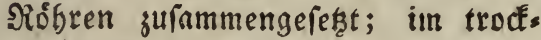
nen Suftande papieribnlich mit uns

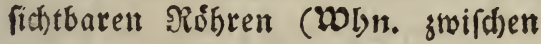

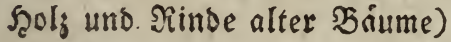

C. 2rseich, fleifhig, lappig, ro̊thlichtgelb, papyraceus, Pas pierlódherpily. unten gelb; bie Socher fehr fein (Wobu. an Baummurgeln)

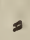

II. Mit einem Strunfe

A. weich, fleifhig;

a ber feut gepolftert,

a flebrig, fattgelbbraun, unten gelb; bie Eofcher rein und ecfig, abge. ftutzt ; ber Strunf furz, bick, mit einem falichen Etrunfringe; Das Flleifh) mildjrabmfórbig

- bulbofis, Dicefúfs

figet. 
B sben braungelb; bie Rsbren ectigt fommerig, fchiefabgeftumpft, grü. Iid)t gelb; Das Fleifd) neifi; Der Strun verfebrt fegelfórmig

b bet f̧ut gemólbt

aeftivus, Som merlocherpils.

I braungelb, unten bellgelo mit rums ben fodsern; ber Ctrun reiflid)t ( $\mathbb{E}_{\mathrm{r}} \mathrm{fd}$. im Sept.)

l u t e us, gelbet:

2 obell braun, unten gelb;

a Der Strum you fdwargen \$unt. ten rauh, unten bicler; ber spilz bicht . - " bovinus, siúbed

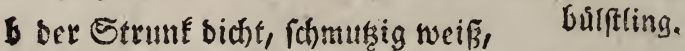
etroas rumlicht; bas fleifa weif̈, unverånderlict) - lapidum, uneÉbas

3 unten ginnoberrotí, fleinlơd)erig; tet Steinbúlfts bas Fleifh gelblicht, att ber Ruft fich fondirgend; Der Strunl furz, sid)t, bick, ròtblicht

- fubvefeus, Etcins

c ber Şut fleifhig, braum, Flebrig; bas búlffling. Fleifch weifß, an bet luft in Slau úbergebento

ling. 
2 oben fantmetartig mic conesutris (d)en Sereifu, unten braun, flein. lócherig.

(WG). alle anf $\mathfrak{3}$ aumfråmmen ober alten Stoffen; fie er: rdeinen vom Juli-Detb. Seiner efbar)

perennis, perennis tenoer.

\section{Borago, నòrigen.}

Die Эlätter burchaus mechelfeitig, bie Relche wegftebent offen (Whln. all Bartenbefrie. bigungett alB flichtling, fonft gebaut. 23ib. Juni, Juli. Gebr. Den Sienen, Epeife) *

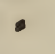

\section{Braflica, Sohl.}

officinalis, gemeis net:

a Die 2 Burgel fohinbelformig; bie S33urgels blatter leberformig; bie Etengelblatter långlid)t ber joơrmig, gejåbut

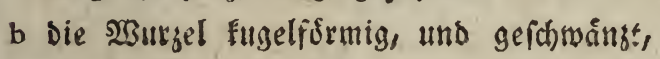
Napus, গeps, Siliveraamen. ober verfefrt fegelfirmig, am obern Enbe flach gebrickt, unb blâttertreibend . Rapa, Rube.

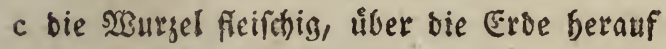
fortfeţend; bie şlåter an ifrem obern Embe fromenfôtutig . . oleracea, Robt.

Gemeinere 2(barten Der Riben.

a bie SYGurgel gans breit, fideiben = ober tellerfơrmiņ
I buxchaus weif

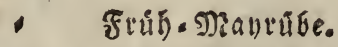
2 úber ber Erbe grůn, , grúnépfigesiutbe.
3 tiber ber Erde roth : , tothtopfige

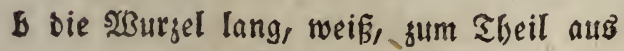
ber Eroe bercorftebeno, freiptsig, reidy, milo $=$ S2 5 c bir 
c bie 23 urgel lang, runo, weifß, feft in ben Эoben vermadjen

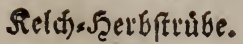

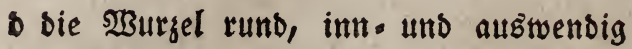
gelb

(NB. Man verwedtisle fie nidft mit gelbe Túbe. ber gelben $\mathfrak{n d g}$ re)

e bie Szurgel flein, furg, berbfleißhig

$r$ angenefimidnedfend

2 nuß̧artig fámedfens
$=$

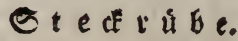

bailifae? mal. Eilifife.

\section{Ëemeinere 21rten des Siohls.}

a Die Blåtter glatt, fopfartig ůbereinander gelegt

\section{I grůn}

2 veilenroth

b bie şlåtter faltig, fraus

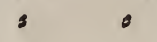

tig, Eraus
I gruingelb

2 rotbblau

c bie \$látter tief, uno fein eingefdnitten

d bie Slumen bilben unerofnet grofie Siépfe,

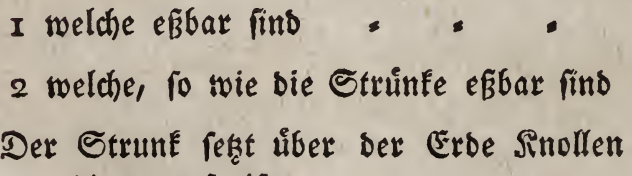
an, bie man ipeifet

f bie 23 urgel unter ber Erbe fopffo̊rmig

(Alle biefe Abarten werben theils auf Aeçern, theils in Gårten gebaut ; man

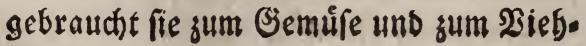
futter.)

capitata alba, weifjes Sinut. capitata rubra, Glaues Rinut.

fabauda, weif́et rBerfing.

fabellica, binuet खBetfing.

felenifia, braunes Siofl.

botrytis, Şlu, mentoly.

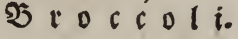

g o n g y lo des, Roblrabi.

Napobraffica, Tov: (d) en, Silumper: เน์bell. 


\section{Briza, Bittergias.}

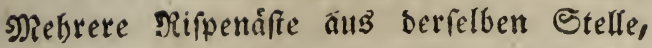
unorbentlid) zertheilt (Wolnt. Sxisten. Zilb) Nav. Gebr. Futter)

media, Biefenjit:

\section{Bromus, seepe.} tergias.

1. Die Rippe ůberbangend,

A. einfeitig, Die Aefrchen vielblithig,

a enformig, mackt; die Bralmen roeg. ftehend; bie untern Slätter benders feits jottis, bie obern feinfaarig fquarrofus, fpat:

6 id)mal, langgrannig, und twie bie rige. ganze \$fange von zarten Scaaren grau tectorum, Dadj:

c Die Branne unter ber Epitze der glat. trefpe.

ten langetformigen Epelzenflappe; bie inmere Rlappe gefrangt; bie Rippe autggebreitet

B. weitfd)weifig;

a bie Aebrchen zufammengebrudft

I breitlicht, 6blutbig; bie Spelgen Inngetformig; bie Granne unter Der Epiţe, länger als bie Spelge grandiflorus,

2 ebfơrmig, lanjetåfnlid); die Epels glob̂tútbige.

zen nadft; bie Grannen furz, ges rabe, ober gefiblângelt : vitiofus, gemeine.

b lanzetformig, 6bletthig, bebanrt; zween Sippenåfte aus berfelben Stelle; bie Grannen lang

- $\quad$ giganteus, Frutters treppe.

II. Die Rifpe etwas ůberbangeno;

2 bie 2lebrd)en långlid)t enforntig

a 4-8bluthig; Die Epelzen bactziegels formig, glatt, biutig geranbet, mit ftuntpfen Spiten, uns Eurgen etwas

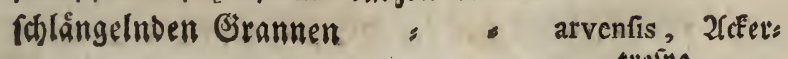
tiejpe. 
b 8-roblúthig; bie Granmen getabe von ber länge bet Spelgeti, bie গiis fpenåfe Eurs

$b$ bie Alfte forram, aus einter Stelle

multiflorus, vicls blutbige.

I alfe fur; ; bie Aebrchen breitgeoruedt, 6-8bluttgig; bie Orannen faft fo lang

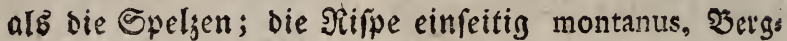

2 bie obern furker; bie Aebrden fleit, tiefpe.

evfốrnig, langgegrannet, nact't, arm. blưthig; Die Siipe aus̈gebreitet

III. Die গRippe etwas aufrecht;

trifforus, Dieub (t) thige.

a die Aebrdien ebformig, mit geraben Grannen, und neb/z ben Slăttern fełr weidhsottig

- mollis, weidfe.

b bie Aebrdhen långlidht enformig, glatt; bie Grannen lânger als bie Spelsen,

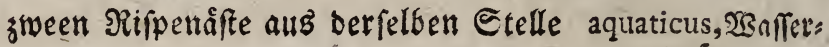
IV. Die Rifpe aufrecht, tiefpe.

I bie 2lebrchen linienformig, 3-7óluthig; bie Girannen Eury; Die Slåtter linien. fórmig

2. Die Aebróen etwas ecfig, fohmal walsen: formig, obne, ober bod mit âuß̧erft furşen Grannen

inermis, Quects tweppe.

V. Die Slưthen in 2lebren;

I bie Aehre úberbangend; bie Albraten ungefåbr 4blutthig, wedbelfeitig, runbs Iid)t; bie Epelgen zugefpişt, furget als bie Brannen - gracilis, fifmate.

2 Sie Rebre aufredt, am Enbe beb f̧als mez; bie Althrchen ungefẳnt I2blưtbig, weçfelfeitig, länglid)t maljenformig, fein bebaart; bie Grannen aus der Epitze

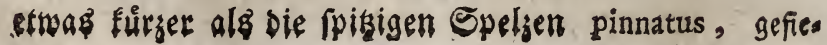
bette. 
3 bie gemeinfhafilidic 2 tebre aus gno ober oreb) vielblúthigen, flachgebrúçten, mit Der breiten Seite gegen ben Şalm ge. febrten 2lebrchen zufammengefesct - diftachyos, fnepi (WOb). Hecfer, Hedertånber, 2̦ie. åbrige. ien, NBege, Nauern. Żlis. Juni. (Gebr. (Jutter)

\section{Bryonia, 3aunrube.}

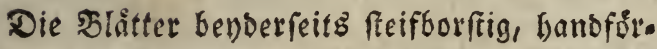
mig, ecfig, ber mittlere Rappen ftârfer ge. fpiţt; Die Soeven fitwars (WObn. in Ge. begen. 23!bj. Nan, Juni. Gebr. Gå̊rs berey)

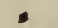

alba, weífe:

\section{Buphthalmum, Sinosauge.}

Die Reldue nactt; bie Slåtter medfelferitig etroas gejainnt,

I rauth, Die Spreublätter bes Fruchtbo. Detts ofne Grante

- falicifolium, neis

2 glatt; Die Eptentititter bes Fruchtbo. Denblátterigeb.

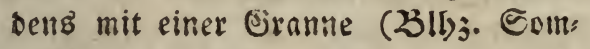

mer)

,

grandiflorumi gro B̈blumiger.

\section{Bupleurum, Şaafeno̊hrlein.}

- Reiut gemeinf(t)aftlict)er Utmidslag; Durch. wad)sblatter (WObn. bergige Gegenden. 215: Juni, Juli)

rotundifoliums

2 ber gemeinf(j)aftlid)e limidhlag etra 5 blåt: rundblåtteriges. terig, Der befondere. 5 blåtterig; die $4 \mathrm{~m}$. (c) Ingblåtter enfơrmig; ble blåtter enfô.

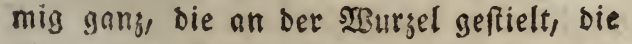

obern umfaffend (Wobn. und 25lb3. mie oben)

longifolium, langs छlåtterigez̆.

Buto: 
Butomus, 2Bafierviole.

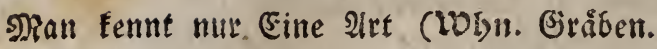

Sllj3. Juni, Juli) $\quad=$ - umbellatus, Dols

Buxbaumia, Sิuxbaumie. senformige.

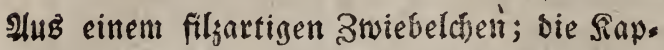

fe! langgeftielt (WDbn. an Fichtenmurgeln; bie Sinpfeln reifun vom $\mathfrak{M}(n)$ - Suni) caulefcens, ges frielte.

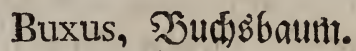

Nan Eennt nur eine wabre 2trt (Lobn. witb

. ftark gezogen)

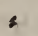

$=$

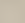

fempervirens, ges meinet.

2 Şaarfórmig

\section{Byffus, Sammetmous.}

I fefre weidh, bleidgelb, sann fich fhwars. zeno (Wont. am Fubboben feuthtftelen. ber Scâluer) = $\quad$ - feptica,fieferdes.

2 aufrecht, golbfairbig, in Alter graumer:

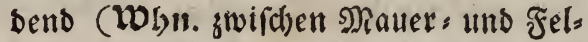
(enrisen, Noofen)

aurea, foftans gelbes.

b feberfórmige, forwimmende fäben in eine

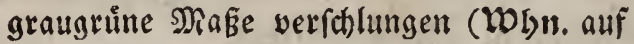
Seen)

c fabenformig, grün, friechend, åftig; bie

flos aquae, $\mathfrak{3} a \mathfrak{F}_{5}$ jetblútbe. 2lefte verfloditen (Wobur. all balbfaulen

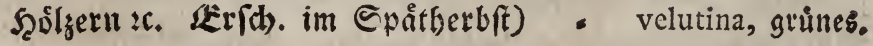

d febr flein, rehr âftig, tief fhwars (Woln. an altem (Semåur) $\quad$ - petraea, Feliens

- Gielbbråunlicht, gallertig; die Fafern vers fammetmoos. worken (WOgn. auf Den Steinen in 23 af. fer)

tremelloides, gals lertiges.

C. Caca- 


\section{C.}

Cacalia, ஒeftrourzel.

Die Sblätter nierenformig, fpigzåtnig; bie Reld)e ungefigr 36 lutthig (WDln. in Den 2llpentbålern. Żlbj. Juli)

alpina, 2llpenpefts wuizel.

\section{Calendula, Siingelblume.}

Die Snamen nachenformig, Erumm, ftumpfo fiac)lig,

I furg, unb swar alle gleid) fo (WDhn. Iecker) * = : = officinalis, ge*

2 bie n̊ußern gerabe, etwas långer, vor. meine. geftrectit (WDlsn. Grióber, gebaut) * arvenfis, wilbe.

Calla, Dractsenwurs.

Die Blatter berfformig; benderlen (jejhlechtg. theile in ber \$lutthe vermengt (WWhn. Wal= Dungen. Blbj. Juni, Juli)
Callitriche, Callitrid)e.

2. Die oberften Blaitter enformig, Die ůbri. gen linienformig; alle vollfommen ganz (Wobn. Gråben. 3lb). Frúbling, Som. mer) * . - . . verna, frúhlings

2 bie oberften $\mathfrak{B}$ låtter ipizig ausgeranbet paluftris, ges meine.

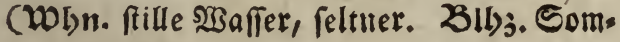
mer, Şerbft)

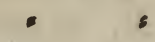

\section{Caltha, Dotterblume.}

callittidse.

a u tu m nalis, Şerbftcallitridje.

Man fennt nur eine $2 \mathfrak{x t}$ (WDbn. an $\mathfrak{B a d}$ hen, Zeichen, auf naffen $23 i e f e n .215$. 2lpril Juni, Gebr. Futter, fút Bienen, Speife) paluftris, geo meine.

Campa: 


\section{Campanula, ઉ3locfenblutte.}

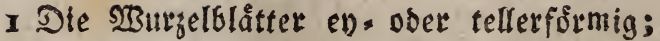
bie Stengelblătter linienformig (1Ob)r. sisiefen; fie bat zablreiche Abarten)

rotundifolia,

2 bie Slâtter ziemlity angedrúfft; bie 2 sur. rundbrítterige. zelolátter lanjetfơrmig, eyahnlid); bie Sterigelblâtter lntzetfórnig; ber Stengel ectig, bebant; Die siifine meitfdreifig; Die Shluthenfticle cinfad); Die Reldhtbeile Degenformig, am Sirunbe zrweyzånnig (Wolv. 23iefen. Z3!bj. Juni) = patula, weiti

3 bie Stengelblätter formal lanjefformig, et: if) weifigt.

was făgegantrig; Die Sield)tbeile wegfteheno; die siifpe ziemlich angebruickt (Woln. 2̧ors bolzer. 3it)j. Juli, 2lug.)

perfic if o lia,

4 bie Sllatter lanzetformig, bie untern eyfór. p fivifichblätterige. mig, geftielt; alle am Sirnde geferbt, unb melfellformig; bie Blüthen gerifpet (IDbn. in (jebegen. 23lbj. Junt. Gebr. Salat) Rapunculus, Tia

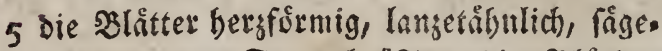
punzel. zånnig; Der Stengel åftig; bie Bhathen lang geftielt, serftreut einfeitig; bie Jeld)s theile zuruectgebogen (IDG). Siebege. DII);. unb Gebr. wie oben)

\section{rapunculoides, trai punjelartige.}

6 Det Etengel eurig;

a bie Slätter geftielt, hersformig, grob. figezaithnig; Die Reldhe gefrangt; bie Slutbenftiele aus breven jufammenge.

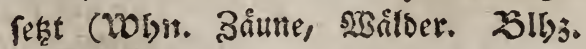
Juli, 2lug.) - . $\quad$ trachelium,

beinfach; bie ßltithen fiellos; bie an ber Eiaut.

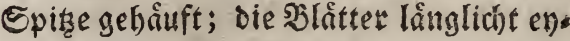
fơrmig, fein făgezsōbnig, bie untern ge.

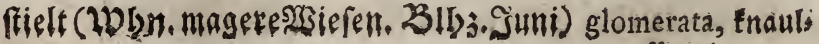
formige 
7 bie Rapfeln 5fiodierig, bedecft; Der Sten; gel ziemlich einfach, beblättert, aufred)t; Die Sluitben aufrecht, einjeln; Die Slume waljenformig mit wegftehendem sande (Woln. (jebege. 2ll)3. 2lug.), Medium, Mas

8 Der Etengel åftig, weitfdhreifig; bie Slåts viette. ter länglicht, etwas geferbt; bie Slutthen einzeln; bie Seld)e langer als bie rabfore misen Sllumen; bie Siapieln mit Sauten (Wobn. 2iecker. 25!bj. Jumi, Juli)

Cannabis, Şanf. $\mathrm{s} \mathrm{peculum}$ Stauenpiegel.

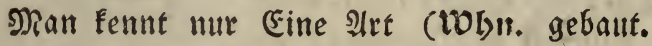
Gebr. Seinmant, Stricfe, Del, Niaftung) fativa, gemeinet.

\section{Cardamine, Sd)aumfiaut.}

a Die Slattter einfach, mit åftigen haaren gefrangt; Die Stengelblätter ungeftielt, lanjetformig; die 23 urzelbläter enformig, geftielt, ichrachgegingut; ber Etengel ein. fad) (Woln. Giebirge. Bllyj. Suni)

b bie Slåtter gefiedert;

bellidifolia, mấs liebenblâtteriges.

a bie șurgelblattchen faft tellerformig;

I bie obern langetånnlid) (Wobn. fend)te Sisiefen. 23lbj. Frübling, Commer. (Gebr. Sconig) * = = pratenfis, 2riefen:

2 bie obern enformig, nur 4 Stanbs. Ereffe. gefåne (Wobu. feuchte Sergnaloum gen. 3lbs. Commer) $=$ = hirfuta, borfiges.

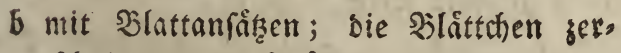
fdinitien; bie Sblutben blumenlos i m $p$ a $t$ i e $n s$,

\section{Carduus, Ctedobiftel.}

Epringtiefle.

A. Die Släter gefiedert serfonitten, 
a in Lenerformige Stidfe; Das Enbfriut grof; bie blithdhen burchaus gleid)

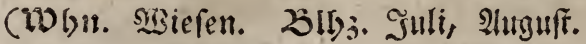
Gebr. Farbereb) *

tinctorius, E(l)ar: tentraut.

b Gerablaufens;

a Die Etricte banbformig, 3-4ifaltig, feinftachlich gefranjt; bie Epitsen ftad)lich; Der Stengal ofen blattlog, einblitbig; ons SBlitbenbaupt ueber. bangend (WhH. Magerer 5oden. 23lij. Juni-Eept.)

medius, langfties

6 unterbrodhen; bie Stuiffe edig, ftad): lige.

lich); Die Şluthen ûberhangend; sie Sieldhfdupen wegftebend, fiectend (Whin. Strafien, Felowege. Zllis.

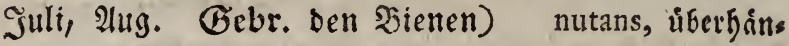

c gekroufet, fachlict); Die Shlithen am gende.

(Ende geniffert; bie Reldyftadyeln nad). gebenb (Wobn. Straffen, Stuinen) crifpus, fraufe:

c umfalfend, fachlid); Die Selchiduppen ftachlid) gefrangt (Wobn. all Bartenbe= friedigungen. 2lb3. Itug.) - marianus, frauens

B. Die Slätter lanzetförmig, berablaufend, Diftel. f(tad)lich gefrangt;

a bie untern gefiebert jerifhnitten, bie obern mit längern uno fưr zåhnen; Die Şlutthen am Enbe ges Drångt; bie Reldffad)eln nad)gebent (Whin. Ufer, gebaute Srte. Slbj. Juni-Sept.) - - perfonata, Rlets

6 Der Stengel armbluethig; bie Sielch. tendiftel. fduppen frachellos (WDhn. magerer \$oden. 2.bj. Juni-2ug.)

defloratus, vers טเน็โte.

Carex, 


\section{Carex, Segge.}

1. Nur Eine einfache långlicht walsenfơrmige 2ebre,

I oben mănnliçe, unten weibliçe Şlus then pulicaris, $\mathfrak{F} 10 \mathrm{f}$.

2 mit gang getrennten (jeiflectitern; Der Şalm borfienformig, abwoirts geftrichen, feinftad)licht (Wobn. 23ies

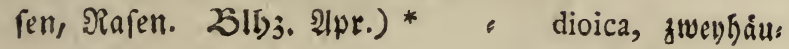

II. Niebrere Aebren. fige.

[ Sende Gefhlechter benfammen; ber f̧alm 3 fantig;

I bie Aebrchen bilben 2ebren, finb ebs: fơrmig;

t ber Salnt oben madt;

a zafilreich),

a rundlid)t, gebraingt, fitellos; an Cirunbe mit weiblid)en, an ber Epitze mit månulichen Sllutthen * " : fpicata, geáfitte.

b bachsiegelformig einanber be: ćend; Die lintern etwas entfernt; Die Slätter fiels formig; bie 2lebre faft 3 tan tig (WObn. feudste rsiefen.

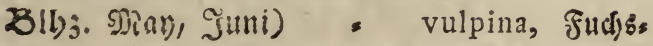

b bidit aneinanber; bie Seldr. jegge. fd)upwen langetformig, am San: De băutig; Der Scalin faharf, am

Grumbe blåtterig ( $\mathbf{S} !$ j). Jumi) leporina, Scafen. c geftielt uno ungeftielt, entfernt; regge. bie ungeftielten in Den 2 daffelit
(Woln. bobe serge)
- axillaris, 2fuferc jegge. d firisls 
d ftiellos ; Der Shalm-an ben Ran; ten feimitadicht;

a bie Alebrdien wenig entfernt, rums; Die Ropfeln fumpf cyformig; oas unterfe Slus thenblatt lanjetformig, forarf zhgefpizt; sie Eduppentaum vertrodivet

$\checkmark$ bie Ilebroben weitlâftig; bie

caneficens, gratu: lidjte.

Sanmengebåufe am Ranbe faum froblid) ; sas unterfe şlutbenblatt enformig; die S. up en moertrodnet (Wun. 2jerge. 23lly. Miay) loliacea, Lolfan's

c die Iefroben zelfreut, wect)= tige. felfeitig lontrglicht, bie in: tern entfernter; bas unterfe Blitbenblatt verfebrt feils fornitg, fdorf zugeipint; bie Sduppen in Der sitte ver= trocknet (10lin. Mnorifte. 3̇!bj. Șแni)

- elongata, verlóns gerte.

+ faft nat́t,

a aut bett fianten oben abwaits feinftadilicht, unten glatt; Die Ieble an der Epitze; die Aebrs c)en evformig genaibert, zer. frent, fiellos, wedfelfeitig; bie Saamenyebrufe am Sande feinftactlid)t, in bet sieife ans?s einander ftebend; das unterfte SIlutbenblatt berjformig; Die Echuppen an Den Seiten ver. trodillet (20bu. nieorige, feuds, te 2 Siefen. ZILj. Juni) muricata, zackige. 


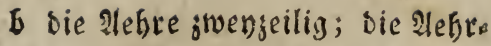
chen linglidist, wechlelipitig, runolicbt, ftiellob, genåbert WWhn. WBaloungen) = brizoides, 3itters

NI3. Carex filiformis. fountent. glabartige.

2 bie 2 ebrathen bilden Trauben;

I bie Traube an ber Epitze, ábren= formig; bie Traubenfticle firryer als bas Slưthenblatt, mérionrig; Der Şalm feillifachlict)t, obell naft;

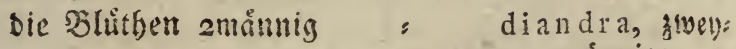

2 bie Tranbe zufantmenthefest; bie mámnige.

Tranbenftiele fo lang als bas 2 li: thenolatt, vielifyrig; bie $\mathfrak{B}$ lutt)chen 3 mónnig; Der Şalm unten ruto paniculata, rifpen:

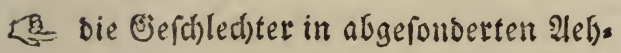
formige. rent.

† จ?ur eine månnlictie;

A. Die weiblitien fiellos, ober boch mit fehr Eurgem Stiele

a bie månnlid)e Megre evfo̊rmig,

a linglicht

I bičer unb långer alż bie weiblichen; weldhe rolgen: firmig, unten gentibert fino; bie Siefchichuppen rpisig; Die P?arben $3^{\text {thei: }}$ lig; bie Siapreln etwas filjig)* : = praecox, fríbe.

2 bie weiblichen fabenfórmig, (Eurs), Die ganje Ringe bes blattlofen Şalmes berab; Die Ståtter langer als bie fralme (WDn. $23 a$ alounger.

23l53. 2pril, Man)* humilis, niedrige. I3 
3 bie meiblichen genáfert, (Eurg), evformig; bie Relch, folupen gerundet, mit bäutigem Siande ; bie ミar. ben 3theilig, Die Sapfeln etwas fillig

ericetorum, Şei: Deregge.

b sie månnliche Albre walgenfót. mis

I lo̊nglidt); Die weiblithen fứzer, aufrect)t; bie 2let). ren weit bon einanber ents fernt; Die Seapfeln fpisizig; ber $\mathfrak{h} \mathfrak{a}$ lm faft runblicht diftans, gettentte.

2 bie weiblichen nabe an ben månnlicheli, faft fuglid)t eyformig; bie Relchichup. pen enfoirmig, lanzetâbn. lict), faft fpitzig, mit eis nem $(\mathfrak{d})$ malen, båutigen Ranbe; Die Saamenges båufe etrwa filşig : montana, V̌ergs

c bie mainnlid)e Aebre gleidfbreit; iegge.

I faft alle ain Ende des ḩalmes ; bie weiblichen faft fugelfoórmig, bie un= terften bev ifrer פeife geftielt, mit bauthigen, zu: geipisten Saamengebăus fen, bie långer fint, als ibre Dedfiduppen (1DLn. feuthte 2 Biefen. Zllyj. Juni) * : = flava, gelóa.

2 bie weiblichen auch gleidt)= breit, långer alB̉ bie månn. liden, unb fie ǔferwach. 
rent; alle aufred)t, nabe alteinanber; bie Siapieln entfernt

- Die mánnliche 2legre lanjet.

digitata, fingers

fórmige. ábnlid); die weiblid)en arm. blutthig, endich bie mónnlidgen úberwadjend; Die Etuizen Der Iebrenticle idheideformig, blattlog (WOLir. Misaloungen. 25lbj. 2(pr. Man) * " nemorofa, Şain:

e bie månnliche Atbre länglidht; regge.

Die wciblid)en lánglidb)t elli. ptifd, gemodfnlid) an ber Epise månnlich); bie unterfte gar viel fuirger alB Das Sllitbenblatt; imo গarben

$f$ bie månnliche 2legre mit ber filiformis, faden: oberften weiblichen alts einer: lev) Stelle; bie meiblichen lángs licht; bie unterfte aus eimer $\mathcal{A}($ ): fel, alle folange als bie mann, lidhe (Woln. naffe $23 a l d o r t e r)$ pedata, fugfor:

B. Die meiblichen geffielt, mit etwas mige.

längern Stielen ;

I bie Aebren fefre fur; Galbbans gend, langgeftielt, fabenfórmig; bie weiblid)en locier; bie fias pieln ăgeipişt; bie Slătter bor: ftenformig (Wobn. Sebirge)

2 bie maimuliche Aebre aufrecht, brachyftachis, lang; bie weiblid)e fabenfơr. mig, ůberfangend, fưrzer als die Stiele; Die Rapreln lang. gefpiz̧t; bie Blatter degenfôr: mig (WWIn. $223 a l$ bungen) - fylvatica, $23 a l b s$ iegge. 
3 bie rweiblicten 2 ebren walsenfors mig, entfernt; bie mánnliche lánger; bie Sthuppen behaart; bie Saamengebanfe ftumpf. (IDLn. Gråben) *

pallefcens, bleidfe.

4 bie meiblichen Aebren fark ents fernt, aufrect)t, zutr Blitheseit linienformig ; bie reifen Rapfelt aufgetrieben, ziemlich frumpf; bie månnliche 2 e bre an ber Epithe elliptifh (MObn. feudhte

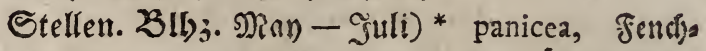

5 bie Aefren lingliclit, fatmal, regge.

faft linienformig; bie reiblichen entfernt ; bie Stiele baarfórmig, faum ůberbangend; bie Rapleln evformig, gefthuabelt, an ben Santen feinftachlicht; die Slåt. ter faft borftenformig : cefpitofa, Rinfers

6 bie treiblichen Aelyen eyfe̊rmig, iegge.

bie oberften faft ftiellob; bie månnliche viel buimner, wals zenformig, von bem 2dafelblatte ber unterften Iebre úberwadhen miliacea, Scirfen:

7 bie Alebren länglidit, waljenfór: regge. mig; bie månnliche (c)måchtis ger, bie meiblichenúberbangend; bie Tiapiéln etwas aufgetrieben, evforrmig

I Nebr, als Eine månuliche; ber Şalm limofa, Sthlamm regge. 3fantig;

I bie mănnlichen 2lebren malzenfór. mig, ziemlid) f(d)meidstig, bie weibs lichen Eurrser, ůberbangeno; ber lange Etachel Der aufgetriebenen 
Sinpiel jwenftad)lid)t (Woin. Seen, Seiche)

2 Die Iefren zientich fhmmidtig, bie veficaria, $\mathfrak{b l a j e n}$ weiblid)en fo lange, als bie mânn: Iichen, aufrectit; ber lange Sdanas bel ber Seapfellt zwevgablicht = bifurca, gablichte. 3 bie Rebren alle waljenformig, ling: lid)t, bie weiblichen geftielt, libers bangeno; Die Rapreln eyformig, gerunoet, f́üger, als bie Relds= ichuppe (Woin. fumpfige $23 i e f e n) *$ flacca, Novafts.

4 bie 2lebren lainglid)t, furggefielt, regge.

fürzer, als bas 2d felblatt; bie obern månnlichen bicfer, vor bem 2hifblüben 3fantig, (Woln, all

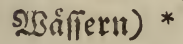

5 bie Rebren entfernt, die månnlis rufa, כitudys regge.

d)en bebaart; bie weiblid)en furys gefitelt; Slatter, Etengel, uno Rapieln bebart ( 1 lyn. fandiger Sioven)

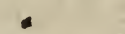

hirta, raube:

\section{Carlina, Eberwury.}

I Neiftens mit fehr ftursem, zuweilen aber mit mebr, alz fubhobem Etengel; allezeit Ibluthig; die Slåtter gefiedert (WObn. .̧eis Den. Blb;. 2lug. Eeptb. Gebr. Epeife, Feuchtigfeitsmeffer) ${ }^{*} \quad$ : = acaulis, grofE:

2 Der Etengel meifens viel-felten Iblütbig; Die Şlumen bilben einen Etrauß̈; Die uns terften blatter gefiedert zerfannitten, bie Etengelblätter lanjetförmig, gezånnt, ftact): licht. (WOlyn. Strafien. 23lt);. Epátioms mer, f̧erbft) *

vulgaris,gemeine. 
Carpinus, Şainbuche.

Die Schuppen ber Råąden flact, (Wolyn. Raubboflacer, 3ilyj. 2lpril, Gebr. Serbe.

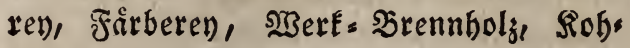
(en) *

\section{Carum, símmel.}

Die cintis befaunte $2 \mathfrak{r t}$ (wolynt auf 2 efern, Wistefen; blílt im Juni, Julli, wirt als

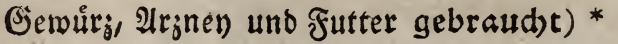

Betulus, gemeine.

\section{Caucalis, Silettentérbel.}

Carvi, Feldéñm nel.

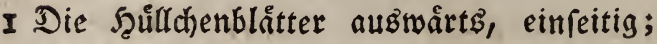
bie Stralblumenblatter febr grof̈, tiefges theilt; Die Ebeile langlicht enfoormig (WOLit. 2leker. 2ll!j. 2lug.

2 Die allgemeine Dolde 2 - felten 3 ftralig, mit grandiflora, grobis blumige. einem einzelnen, abfallenden Umínlags blatte; bie fonderbeitlid)e armfaamig, mit 5 nur außien angeiesten Umíchlagblättchen; bie Snamen mit gezäfnten Angelborften (Wobr. unter Getreib. Z1L)j. Juni)

3. Die allgemeine Dolde 3 ftralig, mit 3 bláttes leptophylla, fleinslátterige. riger, bảutiger şưlle; bie fonderbeitlichen Şưllthen båutig, mebrblåtterig; bie blưs thenftiele feinftad)lidht; Die $\mathfrak{B l a t t e r}$ einfach gefiedert

4 bie allgemeine Dolde 5 ftralig, am Girunde platycarpos, wabie.

zottig; mit 3-5 binfálligen, febr furzen

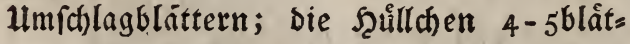
terig; Die Doldchen 3-4faamig; bie Saas men mit gemşbornfórmigen glatten \$ors ften; bie Slätter vielfad) zertheilt (WWhit.

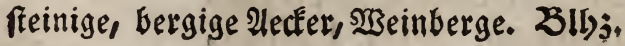
Suni)

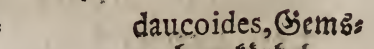
bornétibel. 


\section{Centaurea, flocfenblume.}

I Die Reldfichuppen in eine verbrannte, pfries menfơrmige, ausmårtiggebogene, borftig ges framste Eptize auslaufeno; die slítter uns

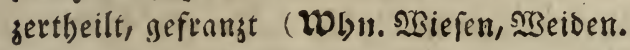
21b). Juli, 2(uguft)

phrygia, phengio

2 bie Teld)idhuppen fägełåbnig;

a Die Slåtter lanjetfórmig, Gerablaufenb; bie unterften gezånt (WDLn. Porge: birge. Z 5 :bj. wie oben). montana, Serge

6 bie unterften 5 litter einfach elliptifd), floctenblume. gezåtnt; bie obern gleidjbreit, am Ranbe gans; Die zuncichft an ben Billithen febr breit, balbgefiedert; bie Ślumen weiß́, rofenroth, blau. (Wobu. Recfer. 25lbj. mie oben. Gebr. Sutter, Den Bienen, Fårberen) *

cyanus, blaue.

c gefärbt; bie Sägegånne vertrocknet; bie Slatter linienfơrunig, unten wollig (WDin. Serge)

anguftifolia, (i) malblátterige.

3 bie Reld)ichupen borftig gefrangt,

a brenfantig, bie sluetgenfơpfden geidup. pet; Die 2̉låtter gefiedert zerfd)nitten; bie Slåttchen langetfórmig, an Der Mits telribbe fteifborftig, an ber Spitze mit einer furzen Giranne; bie Sjlútbenfticle cinblütbig (WDbn. SWiefen, Alefer. 25lij. Juli-Eept.) * =" Scabiofa, Flocken:

b bie ઝlåtter etwas wollig, fablwerbend, fabiofe. gefiebert; Die Sllâttchen gefiebert zers rchnitten; bie Blutthen in einer Rippe

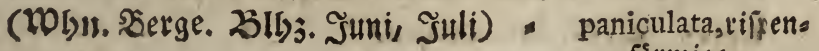
formige. 
c ant Rande verbrannt; bie \$litter gefies Dert zeridnitten; bie Stúdfe vollfomnten gang, febr famal (Woln. Serge. 23lbj. Suli)

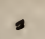

4 bie Relde vertrofnet jerriffen; bie slåts ter langetfisunig,

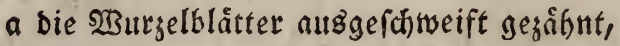
bie Aefte edtig (WObn. MrSiefen, Recfer.

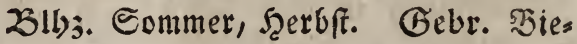
nen) *

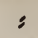

I a cea, gemcine.

b alle ausgefdneift gezånnt; Der Stengel Iblůthig. Wbly. 9gioraft. . 25Ibj, wie oben) *

\section{Ceraftium, Şutnfraut.}

1. Die Slutthe evform: $g$,

I gegeniber, bie obern ftiellos, bie unter= ften geftielt; Der Gtengel anfrecht, wie bie Slâtter, gottig, flebrig (WOLn. ma.

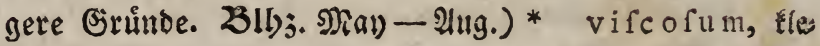
2 raub; briges.

a ber Relth) yon ber fánge ber $\mathfrak{B l u}$ menblatter fehr zottig; ber Stengel jiemlid) einfad), Die જ́látter friellos; (Woln. Straffen, $23 a \mathfrak{l l l}$. Bllyj. Ipr. May)
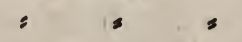

femidecandrum,

$\checkmark$ ber Stengel åftig, unten nieberlie. balbzẹnfádigez. gend (WDlyn. SBiefen, Aecker, Straf, (en. 2SII)3. Snay, Juni)* = vulgatum, gemeis

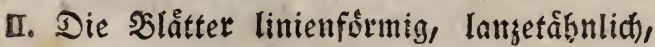
nes. ftumpf, glatt; bie Sllumen gro̊ßer als bet

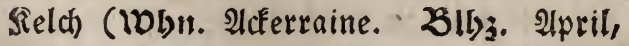
Mav. Gebr. Den Sienen) * 
III. Die \$latter herjformig, gegenúber fiel. log; bie Slluthen einjeln; bie Frichte ab. wåt bangetto (Whln. an Girâben, Duel. [eil zc. 25lb3. Jumi-21ug.) *
Ceratophyllum, Scornblatt. aquaticum, $\mathfrak{g} B n$ s Ferbornfiraut.

Die Blättes gablid)t, (10l)n. Teid)e)

I in 4 3infen getheilet, rauls - demerfum, raubes.

2 in 8 3infen getheilet, glatt . fubmerfun, glats

Cerinthe, SYadbolume. ter.

Die Sllitter umfaffend, gang;

I bie Blume tief 5 fpaltig; bie Theile fpie ķig, geichloffer (WWhn. Alckerraine, 引radjfelder. 23lbj. Stuni. Gebr, Den Siener) * $==$ minor, Eleinere.

2 bie Slunte feicht 5 fpaltig; bie Sheile fitmpf, offen (20!nt. Bebirge. Sib). Juli, 2iug.) - = = major, gů̊sere;

Chaerophyllum, Si̊lberfropf.

I. Der Etengel an Den Belenfen aufgetrie. ben,

I glatt;

a bie șurgst zmiebelformig (IDbr.

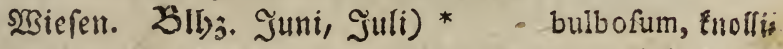

$\checkmark$ geftreift; die 2 latter boppelt gefies ger. Dert; bie lez̧ten $\mathfrak{B l a ̈ t t c t ) e n ~ g e f i e d e r t ~}$ zerfanitten; Die Nittelribben raub

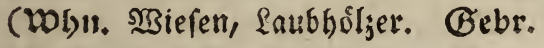
Den Sienen, Piebfutter) * " fylveftre, wilder.

2. raub, rôtflidtblau, an ben Belenfen febr angefdrwollen; bie slätthenftucte

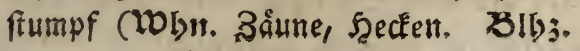
श्रpr. - ذัuni)*

- temulum, beraus 晌enoer.

II. Der 
II. Der Ctengel gleidffơrmig, unb wie bie Blattfitiele, Silótter und SIlumenblätter bes haart; bie Slätter 3mal gefiebert, einges fdnitten, fpişig (WOLn. Jergmålber. 2̧lbj. Sull, Ilug.)
Chaetophora, Şaarmoos.

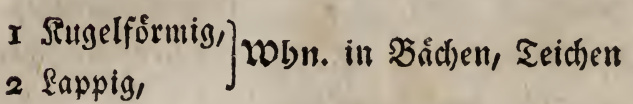
hirfutum, baaris
get.

globofa, fungelfórs miges.

lobata, loppiges.

3 lanjetfermig, ftumpf, einfad) (MObu. auf faulen siuctenfräutern)

Janceolata, Inifiets fórmiges.

\section{Chara, Almleudtete.}

I Die Etengel glatt,

a fracthellos; Die Slätter an ber Spize gablid)t (Whln. (Gråben) - " flexibilis, biegja

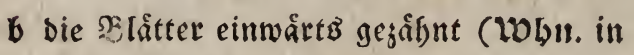

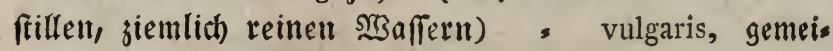

2 ber Stengel unb bie Slåtter fursffachlid)t; net. bie Etadjeln mabe bevfammen (Lobn. Ora: ben. Gebr. Jut treflichem Dung) - hifpida, fteifbots friger.

Cheiranthus, sevfoje.

Die Slåtter lanzetforning,

I glattrandig, frumpf, beftåubt; bie Ed)ot. ten jufammengebrúdt, alt ber Spize ab. geftumpft; Der Stengel walgenrund incanus, Eam

2 mit febt wenigen fachelartigen $3 a \mathfrak{f}$ tt chen, fefre oft, auch gans glattrandig, fpiscig, glatt; ber Etengel edfig , Cheiri, gelbe ßeils (Whit. bende in Birten allgemein. perte. Gebr. A(rgney) 


\section{Chelidonium, Sđjoflfraut.}

Die Shlutthenftiele in Dolden (WDbn. Girten,

Nauen z. 23!b3. Nian- Juli. Gebr.

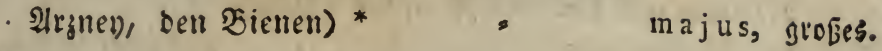

\section{Chenopodium, Şånjefuf.}

I. Die Blätter brebectig

I pfeilfórmig, fonft glattrandig; bie 2 ebren zufammengerest, blattlog, lang. ( $\mathfrak{W l}$ )u. fetter Boden. 2B!bj. Mal - Iuli. Gebr. Speife) * = = bonus Henricus,

2 bersfosrmig, buchtig gełåfnt, fpitzig; bie guter Şeintit).

skluthentrauben aufrecht, zufaumenges

rejt, blítterig, flürer, als Der Etengel * rubrum, rotget.

11. Die Slátter bersfớrmig

I jugefpist, gegaihnt, glánjent, am çrunbe ganj; bie Bluthentrauben in meitláufs tigen, blattlofen গifpen * ${ }^{*}$ - murale, Minuess ,

2 mit fpisigen Effen, uno einer lang ge. gånјеศนี่. jogenen Endefpis; die Slüthentrauben âtî; blattlos (21mm. ben Sd)mei.

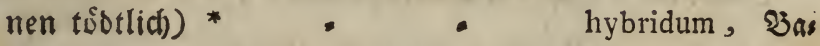

III. Die Blitter rautenförmig frntoginnefuB.

I breneckig, ausgefreffen, am Girunbe gan;, unten meblig; bie oberften lang; bie Trauben aufgerichtet *

album, พveiß̄et.

2 eváfnlid), glattrandig; bie ઝllithen Enculs

formig, in Den Slattminfeln * . vulvaria, ftine

3 buctio gesånnt; bie Mitulátter lanset. tende פdirise. fórmig, faft gally; Die blüthentrauben ăfammengefezt, faft blattlos * - Yiride, gtunet. 
IV. Die Blátter langlid)t

I enformig, glattrandig; Der Stengel uns ten nieberliegend; bie ふlưthen geripet, blattlos, aus ben Blattwinfeln (Gebr. Der Caame jum fifcbiang) * . poly ipermum,

2 Durchaus, buchtig, bie voripringenden Fiidsmeloe.

2isinfel gerunbet; sie Blitbentraubet vieltheilig (Gebr. wiber Miotten, anges nebm ried)ento * = " . Botrys, woblo

3 bie obern keilågnlich, lanjetíbntich, alle riechender.

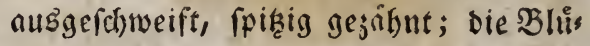
thentrauben einfad), gefaiuft * = glaucum, grauer.

Cherleria, Eherlerie.

Man fentut mix Eine sitr (Whys. Alpett,

2lb3. 2lug. Sept.) $\quad=\quad$ fedoides, feoums

Chondrilla, Éfonorille. nitige.

Die 2 Burrgetblátter geftielt, enformig, langet: åhnlidh, gezâhnt ; bie Stengelblatter ftiel. los, jalunlos, fitmal, an siande zurictiges bogen (Mbin. Iecker. Z3lb;. Juli, Alug. 2umr. Die Pflange ånbert (efbr ab) * . juncea, binfens

Chryfocoma, Jiolbhaar. artige.

Sirautartig; bie Ṡlåtter linienfơrmig, langet. äbulich), bie Reldhe offen (WWhn. Baume) Linofyris, rein: Chryfofplenium, smiljfraut. blätteriges.

I Die Slätter wébrelféitig (IDgn. waiffes rige Drte. Zlbj. Fruibling) - alternifolium,

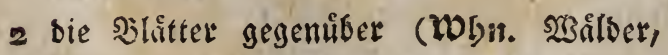
5lb). M(ay))

oppofitifolium, gegenblátteriges.

Cicho- 
Cichorium, NGegrwarte.

I Die Sllithen paarweife, fitellos; bie Slåt.

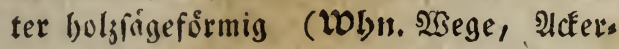
raine. ל̇lb3. Juli - Sept. Gebr. İränev, Epeife) *

2 bie Slüthen einzeln, geffielt; bie Sláttet ganj, geferbt (robir. Giärten. Gebr. in Der Siluche) .

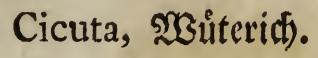

Die Slïtter boppelt gefiebert; bie legten Sjlittchen 3theilig, und einfach; bie Etudfe langsezogen, fcharf lägezåfnig (WWln. an

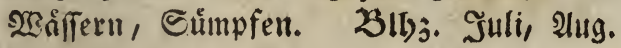
2(min. (ch)äblich) *

,

Cineraria, 2ifchenpflanje.

Die Şlutben in einer Dolbe; bie untern blätter fpatelformig, am erweiterten Theile grob rảgezåhnig; bie obern ganj, unten, wie Die Reldse uno Blutbenftiele filżig (Wobn. Norå̃fte. Sibj. Nay)

Circaea, Scerenfraut.

integrifolia, ganzblatterige.

I Die blätter enformig, juge pist, (d) wachge.

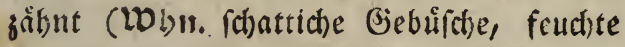
Laubbólger Zlbs. Sulli, 2ug. Gebr. Den Sienen) *
$:$
, ,

Intybus, gemeine.

Endivia, 厄ndivie.

virofa, gemeinet.

2 Die Slitter berzfơrmig, fat)arfgejåbnt
(WDbu. Jebirge)
alpina, 2trpentes xenftaut.

Cirfium, Diftel.

lutetiana, gemeis ก้อง.

1. Die פlátter ftachellog, umfaffend, gefiebert zerid)nitten, gefranzt (WOLn. fendte 2 Sie. ren. Slbs. Juli, 2lug. Gebr. Den Sienen, epeife) *

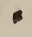

oleraceum, $P$ Bs bate. 


\section{Die SBlattet ftachlicht}

I berablaufens

a gefieber seridnitten; sas anfserfe Etuck Iantetförmig; bie Eeitenftuife alappig; bie Rappen aus einander fies bent; bie Slüthenfunpfe fillyig; Der Etengel bebaart ( 2 bl)m. Etraflen, Felowege. Blyls. wie oben) = 1anceolatum, lans

b thumal, gefrangt, mit vorfaringenden zetfortmige.

furgen Eeitenfúcfen; Das Entoftucté

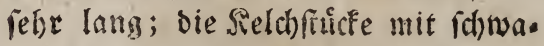
chen Stacheln; bie imern an ber Spitebåntig, webrlog (Wolyir feud)te Gruinde. B!b: Juli-Sept.) *

2 budtitis

paluftre, Eumpfs Diftel.

a bie Reldie foft walgenformig, tubes

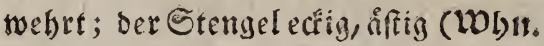
2ec?. Z153. wie oben. Gebr. Futter,

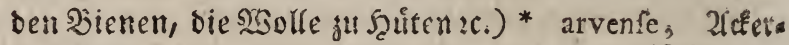

$b$ gefiebert, umfaffend; Der Etengel Diffel. einfach; Die Sblithen an Ende, friels log, von bett oberlten Bloftern bes Decft (10hn. Allpen. Sthy. twie oben) fp in of fit mum, III. Die şlatter gefiedert zerichntiten, ftiels Didffadblid)te. log; bie Sthite wedplelsweife in jween auf eimander geneigten flâchen; Die Jieldje fu: gelfurmig, fadblid)t (Wobr. sisege. Zlbs. mie obent. (Gebr. egbar) " IV. Stengellos; bie Sieldhe unbewefret

eriophorum, wobleopfige. acaule, fitengellofe.

\section{Ciftus, çiftoos@ent}

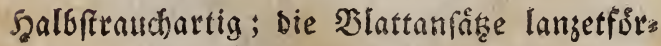
mig; Die Slâtter etwas bebart, länglicht, an ben Seiten gutúdfgerollt (WOhn. Jૃeiden. 23ibj. Ma - 24ug. Gebr. Den \$ienen)* Helianthenum, gemeintes. Clathrus, 


\section{Clathrus, Gitterpils.}

I Etrunkloş, falt fugelformig , sancellatus,

2 Mit einem Strunfe, ber Şut faft lång: ftrunt́lofer. (id)t

\section{Clavaria, Sieulerpilj.}

x. Einfacte,

\section{(Feulenformig.)}

I volltommen einfach) (What. auf abge: fallenen fidtentweigen) " $\quad$ piftillaris, fems

2 bie Etrinfe umgefehrt, puramibenforts pelformiger.

mig, gefreeff, jufammengewachfen, $\mathfrak{r a}=$

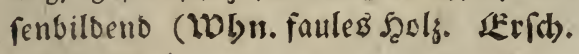
im f̧erbfte)

\section{A. vollfommen ganz,}

elveloides, Fats tempilzartiget.

\section{a. gelb, oben foluppig (Wobnt.}

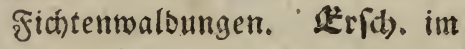

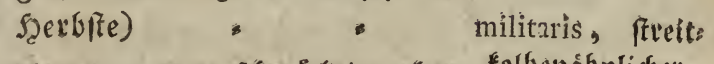

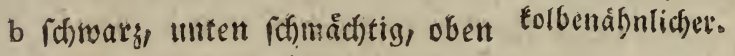
zurammengedrut, frumpf (Whin. şsaldungen, Biebege. (E)rfh. im frübling) " ophiogloffoides,

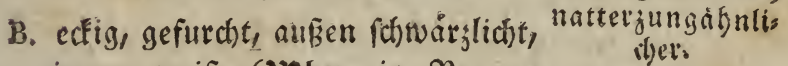
innen weif (LDhn. in Roggen unb andern Gragęhren (Zlmm. (ch)iolich)

C. grin; bie Sieule länglid)t,

D. gelb; bie Sielle blattformig su= fammengedrucft (Mb). abgefals lene Budjenblåtter)

ipathulata, fantels

II. Faft eimfach, oben abgeftuţ, meifis; bie

Clavtis, Diutters forn.

ferpentina, ficlans genfortmiget. aulBerften 2leftchen zufammengedrucft, band. formig (WDbn, in Radelbullsern) - damicornis, ithaufliget. 
III. 2letige,

a rumglidt; bie Spiţent ber leţten Aefte fumpf (隹rfd). int Frúbling)

vermicularis,

b wenig âfrig; bie Alefe prifigig; oraniens rurniformiget.

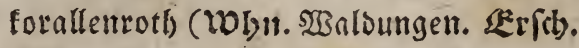
Ilig. - Nov.)

mufcoides, moos: nitiger.

c gelb; bie 2efte gleidh bidf, ain Ende qus

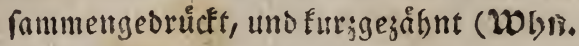

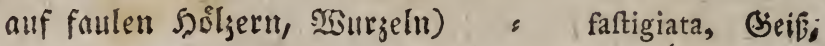

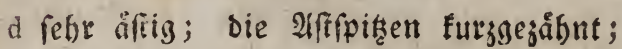
bait.

Der Strumf fefre Dick; bellroth, weif. licht, gelb (WOlnt. WBaloungen. SErfd. Spåtfommer, feerbft) s
Clematis, SZalbrebe.

a Die Släter gefiebert, famt ben Stengeln Hletterno; bie Bláttchen enfơrnitg hergång. lich, gan', oder eingeichnitten (WWly. Şe.

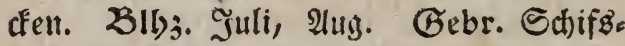
reile, Papier, eingelegte $2(16$ eit) * " Vitalba, gemeine.

b bie \$låtter ungepaart gefiebert; bie Slátts d)en eyformig, lantetåbnlid); ber Stengel gerabe, aufrecht; Die Bllumen 4 und 5 bläts

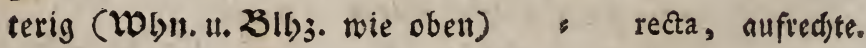

\section{Clinopodium, Doften.}

Die Slåtter enfơrmig, feicht făgejåthig; bie Suirlen in 万ănptchen georångt, borftig; Die Slüthenanfátze borftenforrmig, fúrget alB bie Quirlen (WDhn. Zåume, ḩecken. 2lb3. Juli, 2ug. Gebr. fofflidber Ihee,

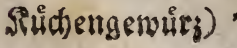

- vulgare, gu meinet. 


\section{Cochlearia, \&offelftraut.}

- Die Stengelblátter linglidft, etwag auš geidineift; bie SWurgelblätter faft teller. formig (Wvbit. an Duellen. $\mathbf{B} !$ b. Juni. Gebr. Epeife, ben Bienen) * - officinalis, gemei.

b bie Stengelolátter lanjetähnlidh, pfeilfórs nes. mig, gej̧âhnt, umfaß̧end

a ziemlid) aufrecht; bie Echiftthen ganj, zufammengedruckt, zfä́d)erig, 2jaanig; bie Sluthen in einen trug= Dolbenáfnlichen Etraune = Draba, Doldenfóv:

b aufrecht, anliegend; sie Sdjortthen migeb. loffelformig, ausgerandet (Wohn. Giråben. Blbj. wie oben) " foetida, ftinten.

c bie Stengelblätter hergånnlid) pfeilfơrmig, Des. umfafient, glatt (WObu. Aecter, wirflid) in Saiern?)

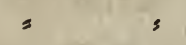
glaftifolia, wau:
blătteriges.

\$ Die Stengelblåtter zerfdunitten; bie 2 wur=

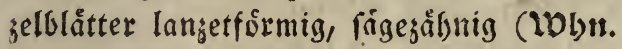

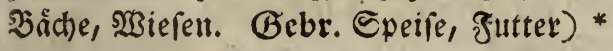

\section{Colchicum, Beitlofe.}

Armoracia, Siten.

Die Slåtter flach, langetformig; aufred)t

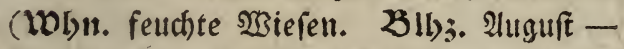
Nov. auth im fruffl. Gebr. wiber unges itefer)*

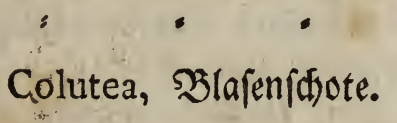

a u t u m na le, Şeetbftzeitlofe.

Baumartig; bie Slåttchen verfehrt bergfơ: mig (WOlnn. (Jånten, aud) wild) * , arborefcens,

Comarum, Sưnffingerfraut. baumattige.

Nan fennt nur Eine Art (WVhn. Sưmpfe. Sll)j. Juni, Juli. Gebr. Gerberev, ben Sienen)*

paluftre, rothes. Con- 


\section{Conferva, $\mathfrak{W}$ afferfeioe.}

E. Gant einfacte, Dưne,

I. gleichformige,

I geglieberte,

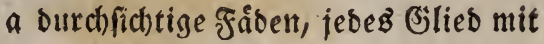

2 långlid)t gepaarten Saamenbåufs chen (WOly, vom 2 saffer verlafs rene Stellen. (krish. im Frůbling) filiformis, faden b in rammetionnliche Naken gebruft formige.

(WDln. Suellen, bie nicht tefries ren. $\mathfrak{E} \mathfrak{r} \mathfrak{d}$. DaB ganje $\mathfrak{J a b r )}$ fontinalis, Duell, 2 ungeglieverte, wafferpios.

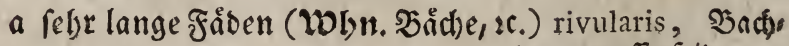

b fefre furze Faben, in eine fungel maflerfetide. frralenformig gefammelt (WWgn. an Noraftpfianzell. Krifd. Juli) Pi fum, Waafies

II. etwas folbenformig, am folbenfórmi. etbie. gen Theile truib; fonft fabenformig, ungegliedert, verworren, burdfifdtig (WDbn. an Fifhen) , " pifcium, Riemen

[2. 2leftige, jeide.

I weidhe, rebre gruine, nicht fingerlange Fáben " " aeruginofa, fwan

2 åıferft binne baarfórmige F̧aben; bie Lefte etwab verflod)ten (Whn. Gräben,

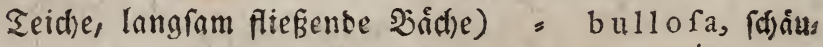

3 felre siftige friten; bie 2 effe in einander grúne.

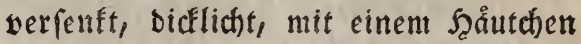
unter einander verbunden (WWln. im (jefundfabe ben Tegernfee) " thermalis, Ediwes 4 ungeglieberte, verflod)tene Fåden; bie felbadronferleibe. jưngern einzeln, âltig (WDLn. Pflangen= aufguำ )
infufionum, 2lufs guẼwafler jeide. 
5 bie Ctånme fafî gablicht, lunten gientict) cinfad), alfwairts kftig (TOL) auf Eteinen it Flingen)

[B. Snotig; Die Gidente linienformig, mit lapidum, Etein wallerperioc.

einem Sinoten am Ende; ein retroriger \$int in feiner Spitte (Wobu, frebende

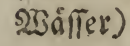

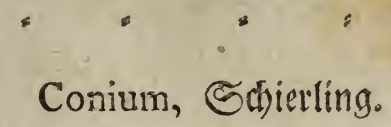

Der Eanme geftreift, geferbt (TVh). Bants tenlant, Aecfer, Echutthaufen, Bămte, an Waftern. Blls. Juni-2luguf. Gevr. Arjuev, fonft fdridlid) $*=$
Convallaria, Mayblume.

I. Der Edhaft blattlog, brenfantig, zwifhen zwen enformigen Sigur

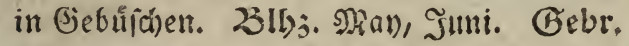
Fårbever)* . . . majalis, getmeine.

II. Die Slötter im Durrt; die Sllétben unter Den Slăttern, bangeno (Wobn. bobe sisals Dungen. 3lb). Juli, 2lug.)

verticillata, quirt. formige.

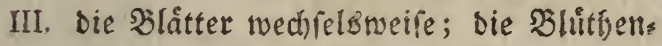
maculatum, ges Hestete. formige. fitele aus ben biattrutnfeln;

a meifrens nur Iblithig; ber Ctengel

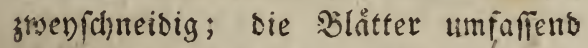

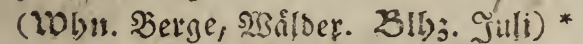

b armbluthig; bie Slatter enformig, zu= olygonatum,

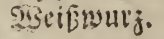
gerpitzt; Der Etengel eafig (WObnt umb 23ll)j, wie oben)

c mebrbluttbig; Der Stengel runb (WVIn.

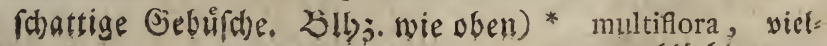
blútbige. 
IV. Die blitter hergformig, furghgeftielt, am Stengel; die Błlumen amainnig (WVbu. u.

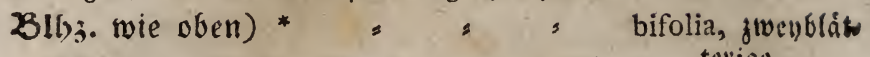

(Anm. alle Manblumen werben febr. terige.

won Sienen befud)t.)

Convolvulus, গ̇inde.

Die Slưthenftiele Iblúthig; bie Slåtter pfeils fơrmis,

I ftumpfipitzig; bie Pfeileden fotharfwitzig (Whn. Heder, sirege, auch) (Biåten. 25lt)3 Eommer. Bebr. mildfwermeb. renbes futter, ben \$ienen) * , arvenfis, 2fife"s

2 bie bintern Pfeilipizen fumpf, faft zu: winde.

gerandet; Die Sllitbenftiele 4 fantig (WWhn. Zåune, Şecfen. 23lhj. 2lug. Eept.) * * $\quad$ fepium, 3aun

Conyza, Dirrwur;.

Die Blötter lanjetformig, 亿pił̧ig; Der Sten,

gel einjibrig; Die Slưtben ftraubfórmig fquarrofa, ge: meine.

Coreopfis, Eoreopfit.

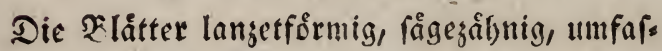
reno, gegenúber (Whan. an 23 affergråben. Zెlb). 2luguft-Eept.) $*="$
Cornus, Dürrlik̨en.

a Die Dolbe add; mit einem 4 blåtterigen Itmichlage; tie Aefte verfdieben gebogen

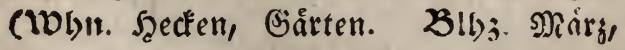
21pr. Gebr. Gerberen, Thee, in bie Stuthe,

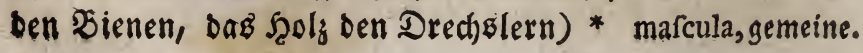

$\mathrm{b}$ bie 
b bie Dolbe unaidt, obne Umidslag; bie Iefte gerabe (IVlan. Gebege. SIly;. Juni. Gebr. (jerberen, Sel, bas Şolz bell

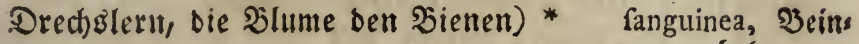
bol?.

Coronilla, Sironwicfe.

a (Ein Strauth; ber Stanm ecfig; bie \$lú: thenftiele ungefåthr 3blutthig; bie SBlumen: någel 3 mal långer als ber Seldh "

b ftrauchartig; Der Stengel eigentlid) bols Emerus, Ecots piontítonniffe. jig,,$\frac{1}{2}$ Spanne bod), nieberliegend; bie Blăttchen evforrmig, eilf an einem Slatte; (bie Dluithen in einer Dolde); bie Şưlfen verengert, berabhangend (WWhn. Scaine. Zli)j. M(a), Juni) * = =

c frautartig; Der Etengel affig, ectig; bie montana, Betg: fronmite. Sluttben in Dolden; Die Scưllfen 5fantig, bev) voflliger Sieife aufred)t, gegliedert; bie 3littchen (ehr zablreich mit einer Ëranne (Woln. Hecfer, SWiefen. Zllj. Juni भug.) * : :

Corylus, Scafelftaube.

Die \$látter enforemigi zugerpight, ungleid) fä. gezånnig (Wolnn. Szecken. Zllhs. Febr. 2lpr. Gebr. Epeife, Del, Dred)Elerarbeif,

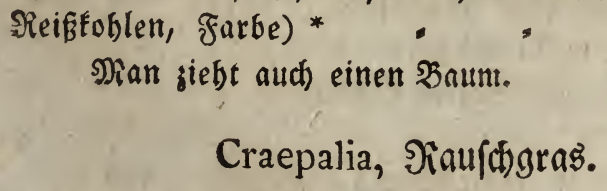

Die 2ebrchen etwas entfernt, mit ङुrannen won ber fange ber 2 lehrchen (Wohn. Alecker, Saine 2c. 315j. Juni. Gebr. (hbriblich) * te mulenta, ge: meines. 
Crataegus, Şageoorn.

I Die slåtter eyforormig, ungleid) fógegritgnig, unten filsig weis (Wolsn. Sebege, Shecken.

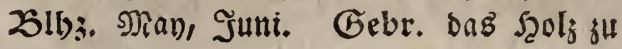

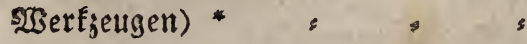

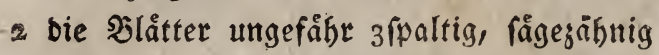

Aria, 2trtegtucet: boum.

a bie Stưcke ftumpf; bie blutthen ein = แn zwentweibig *

Oxyacantla, ges

bie Stůcke fpişig; bie Slưthen burchaus cinneibig *

(Woln. Gende in frecfen. BIl)3. Mat), Suni. Gebr. NRaftung, Futter, Epeife, Brandtemein, Den \$jienelt,

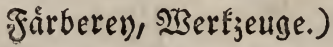

Crepis, Jippau.

r. Der Stengel einfach, armblitterig, arms blůthig; bie Blåtter långlicht, gejånnt, bie bbern umfaffent, bie untern geftielt; bie

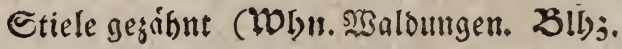
Julli, 2ug.)

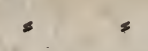

2 bie Slütbenfife farrig, bie oberftent ton ben untern tiberwachfen; bie- 23 Burgelbläts ter boligågeformin; die Stengelblätte: fchmal, langetånnlid), fponoonformig, fitiels log (Żlbj. Niay, Juni) " "

$\$$ Die Blatter gefiedert zerfdnitten, famt Dem åftigen Stengel fteifborftig; bie Stucke gezábnt (Wobn. an sisegen, Ed)utthaufen.

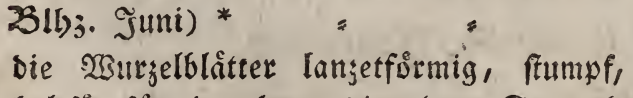
bolzfingefirmig, glatt; bie obern Stengels blåtter ponoonformig; ber Stengel cins fach, oben vielblůthig; bie Rielche etwas sottig (Wobr. Şeiten) * s " virens, grưnet. 
5 bie Stempel, fo weit fie uber bie Strub. beutel Gervorragen, braun; bie blatter lang, fdimal (WDln. STeiben, Felowege, Dåd)er. 23lb3. Juni-Sctb.)* " tećtorum, Dadj,

6 bie Blätter bolzfägeformig gefiedert zer. pippau. fdhitten; bie Stůcke am Sirunde bes obern

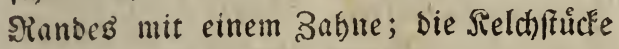
filjis, all ber Mitteltibbe mit meidhen Etad)eln befefat (tDhn. Sisiefen, Garten.

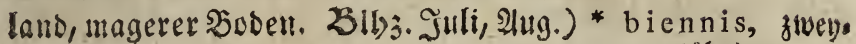
jäbriget.

\section{Crocus, Safran.}

I Die \$arben fafief abgeltust, gang; bie Blite

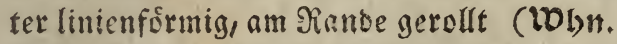
Giarten, aud) wilo. 2315:. Sept. Dct.

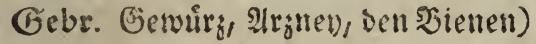

2 Die 2arben lappig; Die \$̧läter begenformig, fativus, SBiente: infiran.

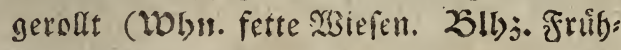

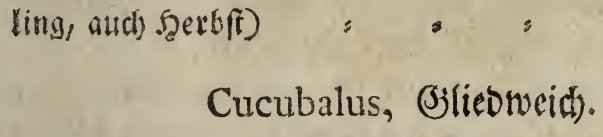

1. Die Şluthen Stwitter; Die Seldbe faft Fu= gelférmig, glatt, aberig, neţåbulich; bie Prigel Der Silumenblatter obrformig ers

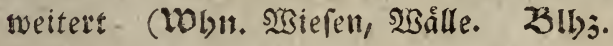
Juni-N/ug. Gebr. Den \$ienen) * Behen, gemeiner.

II. Die Slüthen gong getrennt, zmenhoiufig; Die 2ilumenblätter ungetbeilt; linienfồ. nig; Die Sblatter fwatelformig (WDbn. Fel. fen. Slhj. Sommer) " = Otites, ofutbffe!s formiget.

Cucumis, Surffe, sRelone.

I Die Slattwinfel red)t; Die Fruid)te evfớs: mig ablang, raub = " fativus, gemeine. 
2 bie Slattwinfel fugerunset; bie frithte etwas fnotig, soer rippig

Melo, Melonte.

(Woln. bende in Eårten. Bil);. Sulli.

(Gebr. Speife, Del, ben Bienen)

Cucurbita, STurbis:-

I Die Blåtter hersforemig, weid, feinge. zåfnt, etwas ecfig, filjig, unten am હ゙runbe zmendrufitg; bie fruidfte bolzig

lagenaria, $\mathfrak{F}(a=$

2 bie $\mathfrak{b l i t t e r ~ l a p p i g , ~}$

identuribis.

a rauh; bie Fruthte glatt; bie Caamen mit geichwollenem গanbe s Pepo, gemeiner.

$\checkmark$ bie Frůd)te wargig, fmotig; Die Cchaale

boljig, batt " - verrucofa, wat:

c bie fruid)te cingebrüft, fnotig; ber Eten. ziget.

gel aufrecht $=\quad$ Melopepo, Nies

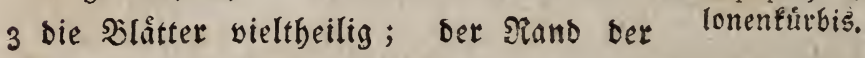

Saamen am Grunde ermeitert $\quad$ Citrullus, STaflers

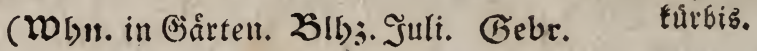

Den Bienen, Epeife, Raftung, Del,

Ariner.)

Cufcuta, Slachşatioe.

Slattlos, fabenfơrmig

I sie Sluthen in Snauln, fitellos, ofne Slưthenblätter (Wolnn. Fette SBBiejen. 23lb). Juli, 2ug. Gebr. (Farbe) * europaea, ges

2 bie blüthen gebåuft, mit Slútbenblät. meine.

tern bededt, sipaltig
Cyclamen, ๔đ)

Der Şlumenrand zurúcfolelegt; bie \$blåtter gerundet, hersformig (Woblt. \$erge. Sllyj. 2(ug. Sept.)

E p i thy mum, Eleine. 
Cynogloffum, Scunbş;unge.

Die Staubfäben firzer, als bie Slume; bie Blătter lanjetfôrmig, grob filşig, bie unter. ftengefitelt, enforntig, lanjetínnlich) (WObn. Felowege. 2llyj. Iuni. Gebr. Den \$ies nen) *

\section{Cynofurus, Sammgras.}

offic inale, ges wóbnliche.

I Die ß̧ưthenblåtter gefingert zerf́thnitten (Wobn. STiefen. 23!l)3. Juni. Gebr. Futter) *

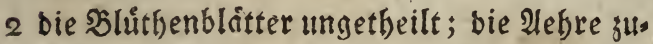
criftatus, gemeis ne?.

fammengesrúct; ; bie åuß̉ere Spelzenflappe mit 3 febr furzen Oirannen an Der Spite (Whln. Sandfelfen. 2llyj. May) "Seffleria, blaues. 3 bie 2lebrchen obne blưthenblatter einfeitig, zwenseilig, fitellos, fteif, ftumpf, angedruift (WDLn. Durrer Soden, Felien) , durus, batteb:

\section{Cyperus, Ẽpergras̀.}

Der Şalin naft, zfantig; bie Aebren gebraingt

I bid)t, ftumpf, lanzetfórmig; Die Dolde 2 blåtterig; bie $\mathfrak{b l u ̈ t h e n f t i e l d ) e n ~ u n g l e i d ) , ~}$ einfach

2 flad), linienformig; bie bolbenformige Rifpe 2-mebrblätterig * . " fufcus, braunes.

(WDin. bende auf feuthten $\mathfrak{B}$ iefen. 2ิlb). 2uguft.)

Cypripedium, Denus(d)ub.

Die $\mathfrak{B}$ Burgeln faperig; Die Blåtter ain Stens gel enfỏrmig lanzetínnlich), zugefpizt (LDbn. in boben Gegenden, frainen. 25!b). NRay, Juni) * . . . Calceolus, gemeik net. 


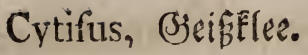

1. Sbaumartig; sie Bhithentrauben einfad),

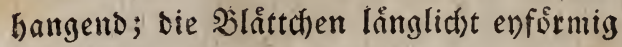
(WOLn. wirb alz Erbjenbaum gezogen. 2(1);. Nan)

II. Rrautartig;

Laburnum, §iee: baum.

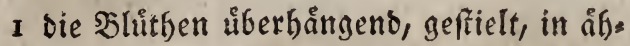
renåbnlidben einfacten, aufred)ten Iraus ben; bie Slattthen lónglid)t enfơrmig

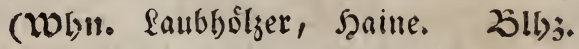
Jumi-Aug.) * = , ' nigricans, iffruars werdender.

2 bie $\mathfrak{B l u t h e n}$ am Enbe ber aweige, faft in Doloen; bie Sielche elippig; die Aefte aufredt) Die Blåttchen eyfơrmig = capitatus, fopfs

3 bie Şluttben aus ben Şlattwinteln; die formiger.

Sieldhe 2lipsig; die Êtengel firaudheno

a nieberliegens; bie Bletthen cints zelm, und gepaart, faft längs des gattsell 3roeiges, auf fehr furgett Stielen (Whur. an Etraffen, mas

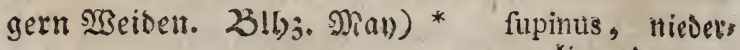

5 weitfinneifig, 4tantig; die Şluthen liegender: einjeln, geftielt; bie relche aufge= trieben, furs, wollemmen glatt (Wol)r. fette Anger. 23!b). Juni, Suli)

\section{D.}

Dactylis, Şunbsgras.

Die Ripe einfeitig, georaingt. (Whbn. Trsies fen, 2 Seiben, நradbacker. Zlbj. Den ganzen Commer) . . . . glomerata, ktruts formiger.

Daphne, 


\section{Daphne, Daphne.}

I Die sluthen friellos, an ben Eeiten ber 3weige, alleseit 3 aus einer binfölligen

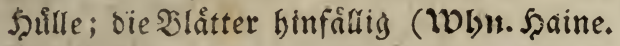

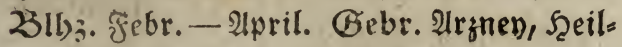
mittel, SRalerfarbe, ben Sienen) *

2 bie Slåtter lanjetfơrmig, glatt
a Die Trăubchen in ben \$lattminfeln
La u reola, gots
beeroanhne.

b bie blutben an ber Spitze, friellog, nn=
Mezereum, Seis Detbait. gebåuft (WDhn. Şeiben)
Cneorum, Steins

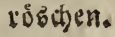

\section{Datura, Stectapfel.}

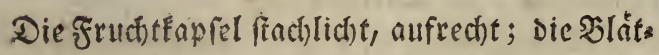
ter. evfơrmig, gejålint buástig, fpiţig, glatt; (Whn. Echutthaufen, Girrten. Slhj. Juli,

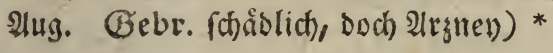

Stramonium, ges meinet.

Daucus, Bjelbrůbe.

Die Saamen rautf; Die Slattfitele unten net: vig; (Wobn. Wiefen, 2nger, Biårten. Gebr. Epeife, Arzney, Futter, ben Sies nen) *

\section{Delphinium, Sitterfporn.}

t Fine cinsige Stapfel; ber Etengel meit. fhweifig iffitg; bie jiluthen zerfireut.

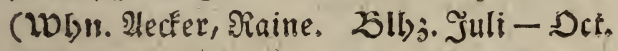
Gebr. Den btenen) * = " Confolida, Felos

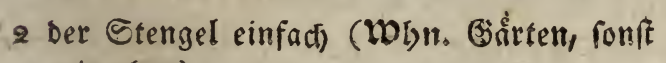
wie oben)

\section{Dentaria, 3afnfraut.}

Ajacis, Barten: litteriporn.

I Drenfache, orenfingerige Slatter (WWh,

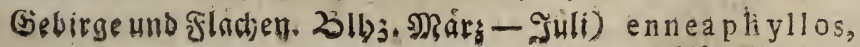
9 Elôttetiges.

2 Die 
- bie untern Şlâtter gefiebert; bie sberften einfach); (h) warze, beerenaibnliche Sollen in

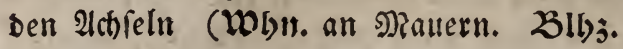
$\begin{aligned} \text { May, Juni) } & ==\Rightarrow \\ \text { Dianthus, Nelfe. } & \begin{array}{c}\text { bulbifera, bollen } \\ \text { tragendes. }\end{array}\end{aligned}$

1. Die Slutthen eingeln;

I bie field)idjuppen lanjetformig, meiftens nur zwo, feltener 4; bie Slumenblätter geferbt (Montr. 23iejen, Straffen)

2 bie Reld)iduppen fpithig, furg; bie şlus deltoides, Felos nelfe. menblitter in baarformige lange Etucfe vielfâltig zerid)nitten; ber Stengel aufs recht (WDGn. Şắlber zc.) .

3 bie Rieldhichufpen febr fury

fuperbus, prådjs tige.

a pfriemenformig geipigst; bie \$llumen. blatter tief eingefdyitten; bie \$låts ter linienfơmig, feinfågezăbnig (Whln. 2Wieien) -* *

plumarius, \$eloet:

$\mathfrak{b}$ evrund; die Slumenblatter gekerbt (WOln. (Järten) : "

II. Nebrere \$lůthen bexiammen; bie Seld). nelfe.

Cariophyllus, Şartennelf́e.

f(d)uppen lanjetformig, folang als ber field), fpişig, und to wie biefer uno die Slătter bebaart, uno nod úberdies freifborfig. Armeria, wilde III. Die ઝlưthēn gehñuft; die Sieldhichuppen Vartneife. evfôrmig, pfriemenformig, gefpigt

I fo lange als ber Sielch; Die Slátter

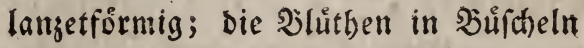
(Wolit. in Den gemeinjten (jầten) * barbatus, fahtme 2 furrger als ber Relch; bie blâtter liniente forrmig, orennervig (WWhn. auf $23 a ̊ l s$

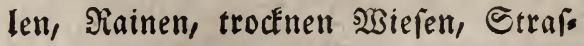
fen zc. Sllys. Juni-Sept. Bebr. ben Şienen, (2)iney) * Sartheujernelfe. Dićtant. 


\section{1}

DiEtamnus, Diptam.

Die Slåtter gefiedert (WOLn. Felfen. Z3I)j.

Juni) $=$ " = albus, weißier.

Digitalis, Fingerfut.

1. Die Reldbblåtter evfor rmig, zugefpizt

I bie Unterlippe Der \$lume inmendig ge: flect't; bie blätter lanzetfơrmig, fåges Jăbnig, unten etwas filzig, geftielt (IDlil. Fandige Oegenden) = purpurea, rother.

2 bie Şlumen punftenlog, bie Slätter ges ferbt, am Slattfitele herablaufend, uns ten raul (LObn. wie oben) = alba, weiber.

II. Die Relchblåtter lanjetfórmig; bie Slåt. ter umfafiento, fágejn̊bnig;

I bie Sllumen behaart, am Rande gottig, ftumpf; Die Dberlippe 2lappig; Die Sielchblåtter ftumpf; bie Blätter enfơr.

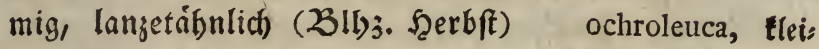

2 bie Slumenlappen fipirig; ber oberfte ner.

2fpaltig; bie \$lătter unten behaart (Woln. Alten, hohe f̧ofljer. Żll);. Suni-Eept. Gebr. alle werben von Sienen befucht, fonft (d)áblich) = ambigua, getber.

\section{Dipfacus, M্জeberfarte.}

I. Die Slätter ftiellog, meiftens zufammen. genactiren,

I rigezátinig; sie Epreuer zwifhen den Slưthchen backenfórmig, furzer als bie ઝlůmchen (Wolt. Moosácker) * . fullonum, ge:

2 bie Epreuter zwifhen ben Blutthchen ge. meine. rnde, linger als bie Blúmd)en (Wolnt. Etraffen, magerer Boden, Gråben) * fylveftris, wilde. 
II. Die Blätter geftielt, meiftens gesibrt (Woln. an Bráben, Sisaffern. Slbj. Juni24g. Gebr. Den Bienen) $=\Rightarrow$ pilorus, belgatk.

\section{Doronicum, Siemsivแrj.}

I Die untern Blåtter fpatelfórmig, weitläufs tig figejafgnig; sie obern lanjetförmig, fitellog, ganj, alle gefranzt; Der frud)t: boden erbaben (23ib;. Juli - Sept.)

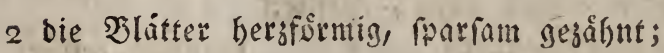

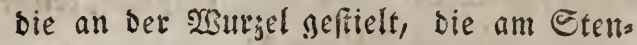
get umfofieno

\section{Draba, Scungerblumben.}

bellidiflorum,

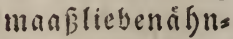
litite.

\section{Pardalianches,} semeine.

I Der Echafit cinfach, faft naft; Die 2 Strzels bläter in eime Sofe geffellt, lansetformig, volfommen gans, borfitis gefrangt; Die Slumen gelb (10bn. Felfen) = ciliaris, geftangs

2 bie Edsifte naft; Die Sisurgelolatter lan. tes. setformig, fwarfam eingefdrutten; bie Slumen weif (WOGn. magere Stellen, aud)

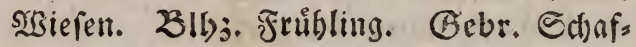
futter) *

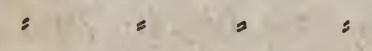

\section{Drofera, Gommenthau.}

verna, Frifflings: bungerblímdjen.

I Die Cdrifte aus ber Srumbel;

a bie 2 latter teller:

fờrmig

$b$ bie Slátter lángs

lid)t $=.1$

Dryas, Waalogotttinu.
WhIt. S) o L i̊fice. rotundifolia,

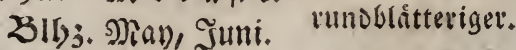
longifolia, lang: bråtteriget.

I(a)t Blumenblåtter; bie Blåtter einfach), ger ferbt (WOLn. Gebirge. 3lb;. Juli)

octopetala, gas manoerartige.

E. Echi- 


\section{E.}

\section{Echium, গatternfopf.}

Der Stengel erhaben punftirt, fteifborftig; Die Etengelblätter lanzetfórmig; freifbor: ftig; Die Slüthenígren aแs ben 2ldfeln; bie Sluthen einfeitig; Der ßlumentano uns gleich) (Whlt. Wsâlle, Strafien, magere Exunoe. 2Jbj. Juni. Gebr. Den SBie. neII)*

\section{Elvella, Şaltenpils.}

- vulgare, gemei. net.

I. Mit einem Strunfe;

\section{Der Ctrunt ro̊hrig,}

a faltig gefurd)t; ber f̧ut faltig, laps pig, abwarts gezogen, " lacunofa, mordjets

b etwas sufanmengedruidft; ber f̧ut flad), ant Rande wellenfortmig juruids gerollt

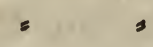

c unrein meiß̄; ber f̧ut unorbentlid) berabgezogen, fdhwars und faltig, unten unrein wei

2 ber Strunf voll; Der f̧ut abroirts ges zogen, fren, unten fnotig; ber Saamen.

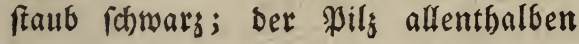
forbars

3 ber Strunt gebogen; ber Rand fraus; pezizoides, Gectiet: pilfăbnglititer.

bie 2 ubenfeite vom Saanenftabe weißs: lid)t; Der ganje Pil $_{\text {s }}$ trompetenformig, fdivary

4 ber $\mathfrak{P i l}_{3}$ verfefrt Eegelformig, trid)ter sifnlich), mit zutrudfigeroultem bicklidhten Sande; inmendig fchwars, aufen afchen. gralt

Cornucopiae, trompetenformis get.

Cruc i bu 1 um, fibmelftiegelfot: miget. 
5 ber gif jiegeltoth, baafenohrformig, oben gerundet, flebrig " = rufa, obrformiger.

II. Struntlofe Arten;

I Eine leberartige, feitruárts auffitzenbe, benderfeits jiemlid) glatte und ebene

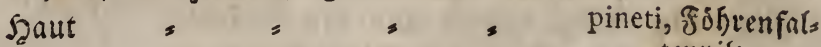

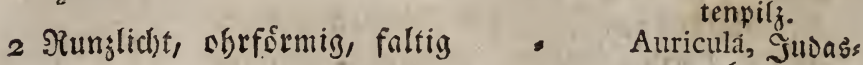

3 23achsartig, feffelformig, wumgelnt, aufs fen weislicldt, immentig bodreth coccinea, garmins (Wolyn. alle in 23 aloungen auf Sins fárbiger.

ben oder alten Eticken. Qkridj. meiftens im Serbfie)

Elymus, Şaargras.

Die Aefre fiemlich aufred)t; bie 2lebrchen glatt, wech)elfeitig, bie unterfen gepaart

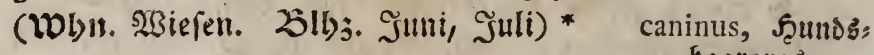
Epilobium, भiseiberiø. bantgias.

Die Sjlátter lanjetfórmig,

I zerftreut; Die Blumenblätter ungleid) (WWhr. altes Gemiuer) * " anguftifolium,

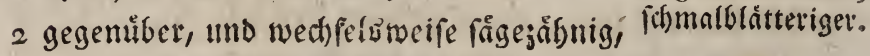
fo wie ber runde Stengel zottig

a umfaffend; Die Blumen grố

b fiellos; bie Plu? 10 Ln. all Gráben. men flein

3 furzgeftielt, gezålont hirfutum, zottio
get.

parvifl orum, $\mathfrak{k}$ leinblumiget.

a Durchaus gegemuீber, enformig, glatt; Der Stengel etwas raut)

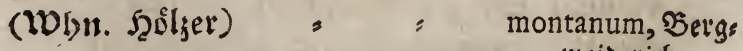

6 nur bie untern gegenuiber; der weiderid.

Etengelvierfeitig (20bn. Sräben) tetragonum, viets jeitiger. 4 亿dmal 
4 fanmal, gegentiber, vollfommelt gants; ber Stengel aufrecht (Wolsu. fenthte Drte) * " " paluftre, Sumpfs

5 enåbnlid), breyfach), gejớlynelt, glatt; ber Etengel 3 fantig

(Sil);. Dutd)auts Juni-Eept. Gebr. iveiberidf.

Futter. Die Saamentwolle mirb von

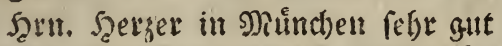
bejablt, und ju friten, $\mathfrak{B a t e n}$ ic. verarbeitet)

Epipactis, Siagwury.

I Die șitzelut faferig, buntoelformig; Der Etengel mit Edheiben befleidet; Die lun terlippe tief 2ffaltig (Wolnt. Wâlber. 2153. Jumi, Juli)* = s " Nidus avis, Glatt

2 Die Sisurgelfnolfen långlid)t, gebåuft; Die trigonum, Drev. fantiget.

Slîtter focheibefórmig, unfaffeno; bie Shus then einfeitig gewunden; bie Unterlippe

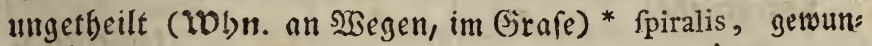

3 brey) zottige Reldhblitter, bie untern $\mathfrak{3 l a t}$. Dene. ter enfornig, nervig, geftielt; bie $\mathfrak{b l u ̈ . ~}$ then einfeitig (Molyn. Sisaldungen. Zlbj. (1แ19.) *

4 bie 2 surgel faferig; bet Stengel 2 blattes rig; bie linterlippe zfpaltig;

a bie $\mathfrak{B l a t t t e r ~ b e r ~} \xi=$ ? formig * = Whin. $2 B$ i e fen. cordata, ferffors

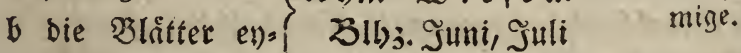
fơrmig * = ${ }^{*}$ ovata, erfortmige.

5. Die 23 urgel faft walsenförmig, nad) allen Eeiten (auch aufwartb) åftig; ber Cten: gel mit anliegenden Echeiden; bie unters lippe 3 fpaltig

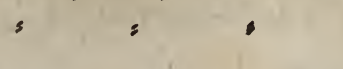

corallorhiza, fos rallenartige. 


\section{Equifetum, Sandelwi}

J. Der Stengel mit einer 2 ehre an Der Spise; bie $\mathfrak{B l a ̊ t t e r ~ a ̊ f t i g ; ~ b i e ~ S l a t t r i f t e ~ b a a r f e i n ~}$ (WDhn. feuchte $2 \mathfrak{B a l b u n g e n )}$

fylvaticum, $25 n$ nld: fandetwija).

II. Der bluifende Etrunf blattlog, mit vers trodfneten, pfriemenformig gefpalteten Schuppenringen;

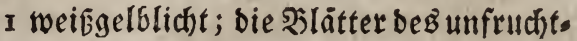
baren einfadb, 6- Io im Duirl; ber

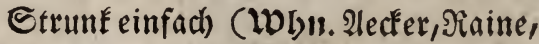
\$Biefen) *

2 Fleinfteiforftig, ant Grunbe oiftig. (Woln. feuchte $\mathfrak{S a l b u n g e n ) ~ " ~ h y e m a l e , ~ S i n n . ~}$

3 gruin; ber unfruchtbare bebláttert, arvenfe, 2lfers. fandeltivijo. klaut. ungefibr 40 Dlátter in Duirl (WDbur.

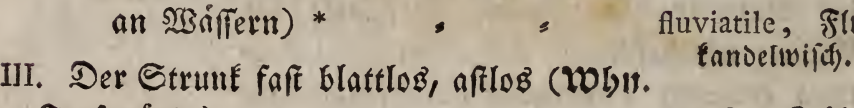
Sorfgruinde)

limofum, Schlañ fandelwilín.

IV. Der Etrunk ecfig, âftig; bie ßlátter cin, fach); zumeilen find mebrere blütheníbren am Ende (IDinn. Griben. Kerids. Der Aefs ren ium Junt. Gebr. ein (häbliches Jutter, aber jum \$uthen ber Metalle, Der Bimmer ze. gut)

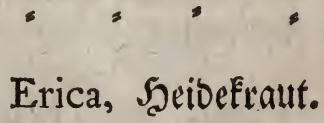

I Die Staubbeutel gmengrannig, innerbalb ber ミlume; bie Bllumen ungleid), glocfenfior. mig, von ber Rånge bes Rieldieb; bie Blát: ter gegenúber, pfeilformig (พDLn. গRabel= bólger, Noriffe. 2̇lb3. Aug. Gebr. ben Sicnen fehr gut, Futter, Gerterey) * , vulgaris, gemei: nes. 
2 bie Etaubbentel feidut zmenfipaltig, unbes welirt, herworragend; bie Shlumen ling: licht enformig; bie Blätter 3feitig, 3-4fad) (WOLn. auf Dưren ঔláten, Eandbergen.

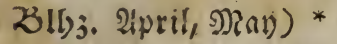

carnea, fleifds: rothes.

\section{Erigeron, Stob Enat.}

I. Det Etengel $I-2$ blüthis

I Der Sielch etwas behant; die Blatter

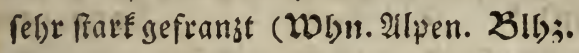
Juli, Ilug.) $=$ = : :

2 Der Reld) Eaum bebaart; die Blatter faft

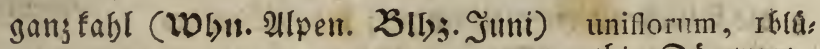

II. Der Etengel riftig; bie Aefte

alpinum, 2rfpens flober:aut. thige Dútrwur'.

I wech felsweife; bie Blithenftiele cinfad); bie Şlitter lanjetformig, galls (10bn. Adétraine, Etrafien 2c. Zlibj. Juli, Slug.) * = s acre, Eleine blaue

2 allfecht; bie Bluthen in Siifpen; bie

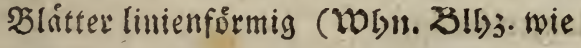
obeen)* $=$

3

canadenfe, canas Dijules.

\section{Eriophorum, MOSollgras.}

I. Der Şalm beblittert, runb;

I bie Slätter (heibentibnlid); nur Eine 2lebre mit vertrodineten braunen ङdups

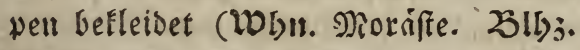
Suni)

2 bie Silatter flach; mebrere Aebren, nach váginatum, rufers Diges. Dem ßluethenftand iverbangend (MDlsis. fumpfige 2 Biefen. Zils. 2lpril-Suni. Gebr. Die Eaamennolle zu Fichern, Bettget, F̧uten, wirb von Şrn. Şerzer in

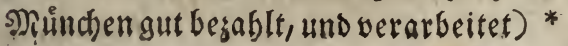

poly thachion,
vielábrigez. 
II. Der Scalm naft, brenfantig, nur gu unterft

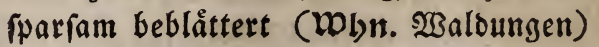

Ervum, sinfe.

alpinum, 2lpen. wollgiaz.

I Die Slutthenftiele einblütbig, lang monanthos, ein. blútbige.

2 bie Sluttbenftiele meiftens żwenbluttbig

I bie Saamen etwas sufammengedrúctit

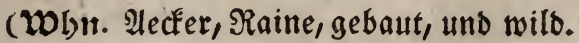
23lyj. Sutli. Gebr. Speife, aber bart gu verbauen) * $\quad$ = Lens, gemeine.

2 bie Saamen fugelrund, 4 in einer Scưlie

(Wobn. 4. 2llbj. wie oben) = tetrafpermum,

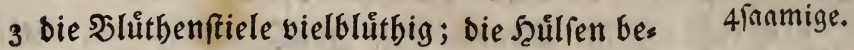

- baart, 2faamig (WWln. h. Zibj. wie oben)* hirfutum, gwels

Eryngium, Mannstreue. jaamige.

Die slätter umfaffend, gefiebert zerfdhliffen (Won. ungebaute Srte. Ṡlb3. Juni) * campeftre, Fetb. Eryfimum, Şederid). mannรtteue.

I. Die ミlätter langetfơrmig,

a gezáfint,

I won nieberliegenden Şanren graus licht; bie Relche burchaus gleichfårbig

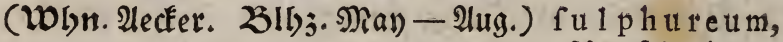

2 bie oberften Stengelblatter falt lis inbefelgelber.

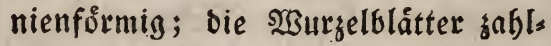

reid); bie Selche an ben Spitzen bun=

felfárbig (WDbur. Berge. Slbj. Nay) hieracifolium,

6 solffommen gals, mur mit fparfamen, Gabiditfiautblats vorfpringendell Żiłnen; Der Stengel ecfig (IVIn. Necfer. WIbj. Mian, Juni) chei ranthoides,

11. Die Slätter tellerformig, berzånnlid, ges lafblumenartiget. zågnt (WWh. Gebuifhe, OGftgårten. Z3!bj. 2(pril, Mada)

Alliaria, faudfat: tiget.

III. Die 
III. Die Blatter lenerfórmig; Dab álnerfte Slåttchen faft tellerfórmig (WDlsir. feuchte

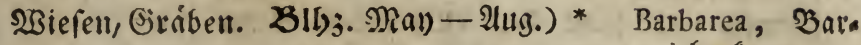

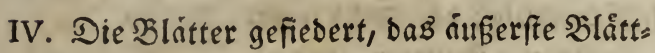
bentialt. d)en felgr grob; fpondonfórmig; die Schos ten aufrecht, angebruickt (Whn. Straffen,

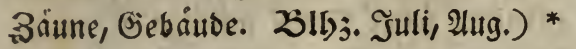

o flicinale, ges wófnlicher.

\section{Evonymus, ઝfaffenÉąpel.}

\$. Die Slåtter geftielt, eyformig langetånn.

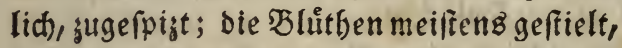
meiftens 4 mannig; bie Siapfeln ftumpffan. tig; Die jungen ameige 4ecFig (Woln. Şes c'en. Zil)j. Man, Juni. Gebr. S(f)ubn: gel, Drechslerarbeiten, Der Sname Del) * vulgaris, gemeis

2 bie Slatter enformig, gang; Die शarbe ttes. fopfifnlich; bie jungen 3weige rundlid)t; Die Rinbe glatt

\section{latifolits, Eveits blátteriges.}

\section{Eupatorium, Eupatorie.}

Die Blątter gefingert (Wobn. an ufern. 25!bj. Juli-Eept. Gebr. Man fonnte fie wie Şanf benư⿱宀㠯en) * : cannabinum, நanf: aitige.

\section{Euphorbia, 930 (fริmild).}

1. Die Şauptbolbe befteht aus 3 Stralen; jeber Etral trågt 2 Doldchen.

I bie Umiblagblitter enfórmig; sie Slat: ter gan berfebrt evformig, geftielt (Wobn. Sartenland. Bllyj. Sommer)* Pe plu s, runde.

2 bie Ulmidhlagblatter langetformig; bie Blåtter linienfớrmig (WOIn. औecter. 25lbj. wie oben) ,

exigua, Fleine. II. Die 
II. Die J̧auptbolbe 3-5ftralig; jeber Stral mit 2 Dolbd)en; Die Umichlagblatter en: formig, und famt ben elliptifden Sten.

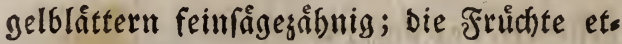
was uneben (Won. fteiniger boden. 23lbj. M(av, Juni) $=\quad=\quad$ duicis, fúbe.

III. Die frauptbolde 4ftralig; jeber Stral mit 2 Dolschen; bie Slitter gegeniber, glatt: ranbig (Whn. Raine, aufgemorfene Şügel) Lathyris, fprings IV. Die frauptoolde 5ftralig; Ermutartige.

I jeber Stral mit 2 Dolbchen;

a bie Umichlagblätter ber Sauptbolde lănglidfteyformig, jene ber Dolodjen berjfơrmig, Rägegábnig, vollfoummen getrennt; Die Slâtter fpatelfórmig, fágejågnig, bebaart; bie hapfel wars zig (WWlit. fetter 5oden, 2jract)= åcfer) *

b Eurrzer als bie lumfallagblatter; bie cordata, bergs búllige. Slumenblátter gan; ; Die Slátter wedbelfeitig unt evformig; wie bie Hlmichlagblátter (Bll);. Sommer) hirfuta, jottige.

c Die Doldchen wieber zfralig; Die Llm: fhlagblatter ber frauptoolde enfơr, nig, jene ber Dolbchen burdiwachs: blâtterig (Wobn. Trsaldungen an Straffen. Zlb). 2Atg.) = fylvatica, $23 a l o$

2 jeber Stral ungefăbi mit 3 Doldden, wolfšmil๘).

biefe wieberum 2ftralig; bie blátter lanzetfórmig, fågez̊ånig

a bie Umíchlagblátter Der Doldchen ev): formig; bie Frudbt ftarf, uno unors bentlich warsig, oft zottig sugleid); Der Griffel stheilig " $\Rightarrow$ verrucofa, zige. 
b bie ltmidhlagblitter ber Dolbchen hers: formig, am giticten bev Mittelvibbe oft banig; bie Fruidtete war;ig, mit glntten Bwifd)enftrid)en (23lb). Juni, Juli)*

3. platyphyllos,

c bie Llminchlagblatter ber Doldochen ver, beritblätterige.

febrt enfơrmig; bie Blåtter feilfór= mig, fägejådnig (WWlyn. Bartenlano,

Dradbåcfer. 21b3. Sommer) = heliofcopia, fon:

V. Die Şauptbolde vielftralig; bie Seitent = nenmendige. afte unfruchtbar;

I jeber Stral mit 2 Dolbchen; bie Utnts fchlagblatter ber Dolddien faft berifor. Inig; bie frudht glatt; Die \$llåtter li. nienfornig. (Whn. magerer Soben, șålle, an Ctraffen. Z\$b). Juni, Julli. Gebr. Farberey) * = Cyparifeas, cys 2 jeber Etral mit 3-4 Doldchen; sie preffenartige. Frud)t warzig; Die Blatter lanjetfór. nig, fitelíos, meiftens volfommen gans (Błbj. Nav, Juni) = = paluftris, Sumpf Euphrafia, 2fugentroff. wolfsinildis:

I Die Slatter eyfơrmig, geftreift, ftumpf,

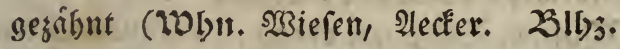
Juni-Cept. (Gebr. Futter)* officinalis, gemeir

2 bie Slátter id)mal lanjetformig net.

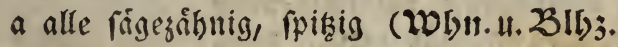
wie oben * $* \quad=$ Odontites, brom

$b$ mur bie untern fögezånnig; bie obern ner. volfemmen ganz; bie Slumen gelb:" 1 u tea, gefber.

$$
\text { F. }
$$

Fagus, כ̧ude.

Die Slåtter enfơrmig, unfenntlic) fågezóth. nig; bie jüngern mit scăaren gefrangt 
(Whit. SYBalbungetr. 23l5; Mav. Gebr. Das $\mathfrak{a} a b$ zum Sierben; Det Saame jut

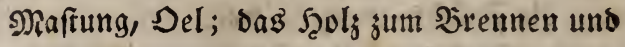
zu Soblen. Eine Ant beift Weif̧budbe, bat weiferes Solg, belleres $\mathfrak{a} a$ b, und bläfern Saamen) *

fylvatica, Sioth: (buá)e.

\section{Feftuca, ๔đwingel.}

1. Die Sippe einfeitig;

I Die Aebreten mit Grannen;

a bie Siifpe verengert, bebaart; ber Şalm oben vierfantig; bie Slatter pfriemenfórnig (Whit. magerer $b_{0}$. Den. Slb3. Man, Juni. Gebr. Schaaffutter) * *

ovina, Eciaaf:

b raut); bie Aefrchen 6 blůthig; bas idfwingel.

leşte Silůt)doen grannenlos, bet

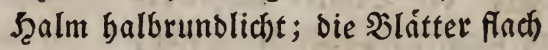
(Wolu. L. Sll);. wie oben) - rubra, iother.

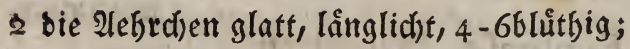
bie Slåtter borftenformig eingerollt; bie \{iippe lanjetfórmig; Der Şalm burd)aus rumblidht (WWln. 4.23 l)j. wie oben) duriufcula, நanter.

3 bie 2tehrchen gegramet, ober grament los; runt, 6-8blutthig bie Sitipenåfte aufgerichtet, faft anliegent (20)n. 23 ies fen. 23lbj. wie oben. Gebr. Futter

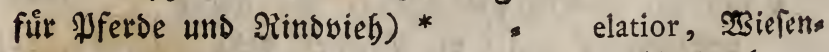

II. Die Milpe fiemlich einfach, aufgerichtet, (d) roingel. faft anliegend; bie Alebrdhen gramnenlos, armblưthig; bie obere Rield)tlappe faft lån. ger, alb alle Slâttd)en jufammen . decumbens, lies gender.

III. Die 
III. Die Siipenåfte serftreut, alfrecht; bie 2lebrd)en faft ftiellos, unberwebrt, anges bruidf', vielblütbig, rund; bie innere Spels zenflappe jwevipaltig; bie åniere abges ftumpft (Wobr. frille, reime Şaffer, Grå= ber. 5lb: wie oben. Gebr. Der Gaas ne jur อiruge $2 c$.

fluitans, Edywo Denid)wingel.

Filago, siufreffraut.

I Die Şlithen in ben $\mathfrak{W}$ infeln und an ben Episen Der 2lefte, in faft fingelformigen Scaufdien; Der Stengel gaflicht; Die 2lefte parrig; die Slätter prisigig, filsig (Wolt. Hecker, \{aaine. Zうbj. Juli, 2ug.) * " germanica, beuta

2 Die $\preccurlyeq l$ lutben aus ben Slattwinfeln, an ben iches.

Seiten, ftiellos, fegelformig; Der Stengel aufrecht, zertheilt; bie Slåtter mit einem ftartwolligen fillye (WWhn. Dưre \$låke. 231b3. Juli-Gept.)

montana, כeergs

3 Die Slüthen in ungeftielten Sơndeln an tubtfitalt.

Den Enden Des Stengels uno ber Aefte; ber Stengel aufrecht, åfitig, wollig; bie Eamien mit einer Şaarfrone (WObn. unter ben Stoppeln. Blij;. 2lug.) *

4. Die Slluthen am Ende gedrångt, swifden arvenfis, 2fater: tublétrat.

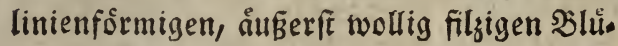
thenblittern; Der Etengel gan einfach (WObn. bobe Felfen. 25lb3. 2lug.) " Leontopodium,

\section{Fontinalis, Şůllmoobี.}

Die Ståmme fd)wimmend; Die Slåtter ens fơrmig, Lanzetåhnlich, růưnortb vertieft, einworits erfaben; die stapreln aus ben

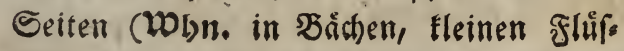
(eii)

- antipyretica, grof กิยื.

Fraga- 


\section{Fragaria, Erobeere.}

I Ranfend; ber Frudtroden wird fleifhig (IDbn. III 23 aldungen. 25l5;. 21pr. - 2lug. Gebr. Speije, Arzuey, den Bitenen, Ger= beren) * " " " vefea, semeine.

2 Rantenlos; Der Etengel niederliegens, twurgelno; der Futchtboden faftlos (WObur. bobe Giegenden. 2lbj. Juli)* = fterilis, taube.

\section{Fraxinus, Ejole.}

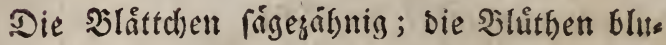
menlog (20bir. Raubbóljer, faaine. 23lbj. Apr. Gebr. Das Saols fommt bent Eichens bolge gletch, gut eingelegten = uno Drebarbei= tell, abnelt gebeigt Dem Nabagonifol $l_{\xi}$ * excelfa, gemeine.

\section{Fumaria, Eroraud.}

I Die wisugel fitollig,

a meiffens hobl; Die blůthenblätter lan= zetförmig * " = " bulbofa, Enollis

b niemals bohl, fonbert bidht; sie Shlis get. thenblatter gefingert; Der Stengel ein fact)

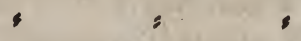

digitata, fleiner.

2 ber Etengel weifforneifig, febr âftig; bie Slätter vielfach zertheilt; Die Stúcfé zus gerumbet, lanzetformig; Die Şülfen ein= faamig

(IDbur. Nodererbe, Getrege. Zllj;. officinalis, gemeis 21pr. - Juni. Gebr. Den bienen)

\section{Fufcina, Gjabelmuno.}

1. Der Ctamm blâtterig gefiedert,

I ganj einfad); bie Slätter gul benden Seiten simjeilig 
a bie Fruchtftiele aแs่ Dent Girunbe unter ben frifd)en Slåttern (WDyn. (d)attid)te Scedfen) * " taxifolia, eiben.

$b$ bie Frudtfftiele aus Der Spiţe blátteriger. sber Den frifchen Sbláttern, bryoides, gefieder:

2 éftig; Die Slátter benderfeits znenzei= ter.

lig; bie Rappelfitele aus bem Crunde (Whn. wie oben)

$\therefore \quad$ :

II. Die Ståmme jitemlich aufred)t; bie Slåts ter linienfirmig, fpistig, faft fidselígnlid), nach einerlev) Seite geridhtet; bie Saprel etwas gebogen; Der Dectidnabel lang

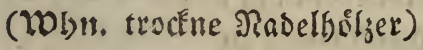

III. Det Etengel friectient; die Aefte auf. fcoparia, befents formiget. red)t, zmeigig; bie 3weige jiemlid) aufs red)t, runolicht; bie Slåtter breitlid)t, fcharfipisigig; bie Siapfelftiele zmeigelang; bie Rapieln aufrect) (Wobn. an Saum: wutrzeln)

IV. Die reifen Sapreln enfortmig, trichomanoides, wiederthonattis get.

a gefteift, etwas überbangend; bie Strimms chen gablicht; bie blätter pfriemenfơr mig; bie Frudtffiele aแв ben Geiten (HOlnn. feuchte $23 a l b u n g e n)$

- purpurea, purputs

b Die Rapfeln enförnig; bie Dedel id)nas fciurides, mailles fiswanzartiget. rotbet.

belformig, frumn, faft fo lang als bie Saprellt; bie 3tweige ant Ende äftig; bie Slitter bachsiegelformig, langetígn. (id), zerbrecblid)

glauca, glaugrí: nei.

V. Die Rapfeln überbangend; bie Strimm. c)en aufiect)t, gebrängt, rafenbildend, ziem: lid) eimfach); bie Blåtter borftenfơrmig; bie Srudtftiele aus ber Ditte (WDbn. nabe bey Duelfen) c)er. 
VI. Die Slåtter einfeitig genteigt, fichelåhns lid), pfriemenfo̊rnig, aus einem båutigen Girunde; Der Sapfeldectel fchnabelformig (WOLn. $2 \mathfrak{s a l d u n g e n )}$

$$
\begin{gathered}
\text { heteromalla, eins } \\
\text { peitiger. }
\end{gathered}
$$

\section{G.}

Galanthus, Gdjneeglicedfen.

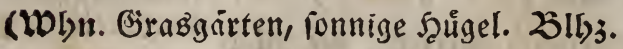
Febr. - Apr. Gebr. Den Sienen) * nivalis, gemeines.

Galeopfis, Sooblialin.

I. Die Şlâtter Lanzetånnlid linienfơrmig; bie Dberlippe ber Slume linienforrmig, an Der Spise orevgrăbnig; Die Stengel am uriprunge ber 2 lefte aufgetrieben (WDbu.

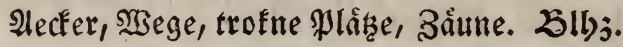
Suli - Dct.) * .

II. Die Slätter enfoirmig

Ladanum, iffmals blâtteriget.

I fpiçig; bie $\mathfrak{b l u m e n}$ nod) einmal fo lang, als Der Reld), Rishig; Der Stengel an ben Gelenfen fnotig (SSie oben) * Tetrahit, Tes

2 bie Blumen viermal fo lang, als ber

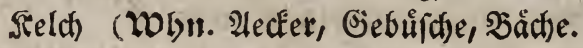
25llj. wis oben.) tiabit.

\section{Galium, \&abfraut.}

I. Die reifen Fruid)te glatt (Jin Der Sugeno fino fie mandi)mal raub)

I Die நlåtter sierfach, langgegogen, un, gleid), (fifmal, ftumpf; ber Stengel vier. fantig, an ben Santen, wie bie Slatter am Rande, feinftachlid)t (Whrt. feuch)te

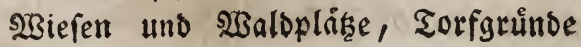
25(b). Juni 2c.) . . !

paluftre, Sumpf: IabÉtaut. 
2 bie Stengelblätter meiftens fünffach); bie 2litslatter vierfach; alle etwas breit, licht, faft linienfơrunig, glatt, am Sianbe feinftachlid)t; ber Etengel (fth wad), viev: fantig, an ben Santen feinftachlicht

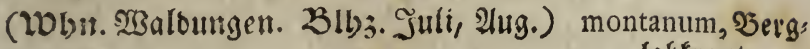
3 bie Slåtter 6 fach), Ianjetforemig, labfinut.

a mit einer furzen Stadbelipitze, am Sande mit bafenformigen Etacheln (Wolnn. feuchte sjiefen, Moorerde,

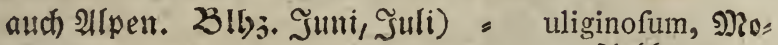

b am Rande feinffachlicht; bie Snoten raftabtitaut. einfach); bie Fruid)te in ber Jugend etwas raulf; bet Ctengel (d)wad),

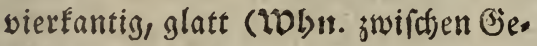
frnoltchen. 23!b3. wie oben) = fpurium, neífes.

4 bie \$loitter 6-8fach, mit einem Stachel an ber Epise; bie untern eyformig lan: zetáfnlid, bie obern lanzetábnlid), lis nienfơrnig; bie Blithentuifthel aus ber Epise, orevtheilig, gablicht (wown. Al. pen. 23lly; Juli)

puffillum, fleinez.

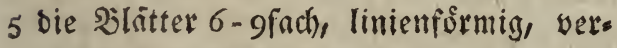
febrt, lanjetrifnlid), feinftachlid)t, zuge: fpist, glattrandig; bie untern feinftachs licht gerandet; Der Stengel glatt, viers fautig, am Cirunde geftreft (WDhur. fteinige $\mathfrak{B a l s u n g e n ) ~}$,

6 Die Sllátter 8fach

fylveftre, Şain: laberaut.

a linienfơrmig

a Ianzetåfnlich, fcharfipitigig, gans megftebent, glatt; Det Etengel (d)wach), glatt; bie 2lefte wegftes beno, viertantig (WObit. Şedtert,

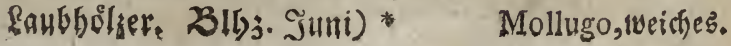


b Inten stweumal gefurcht; bie blis. benden 2lefte furs; bie Sllume gelb, auch) weir (WDbn. Raine. 23193. Juni-Eepr. Gebr. Futs - ter, fárberen)* : " verum, gemeines.

b langetfismig, zugefpigt, feinftadjlid)t fî̉esôbnig, glatt; bie blitben aufs recht, bie Fruchte liberhangend; bie Blumert gelb (WDLr. fteinige berge.

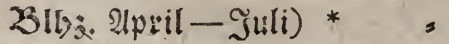

c bie Slüthenblatter gegemúber, paar. minutum, Heis neछ. weife, alle verfebrt enformig, langets åfulid), zugeipist, am ?anbe feinfachs

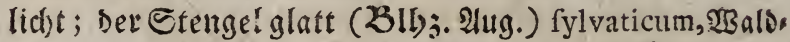

7 bie Sllatter 8-1ofad), in Suirlen, li. labtinut.

nientơrmig, an ben Eeiten zuruefges rollt; ser Etengel unbewebrt;

a bie Bluttenftiele swewgablid)t, an ber Spitze Des Ctengels; sie găıe Pf. blåulid)tgruin

glaucum, meets grưnes.

b bie Slüthemftiele orengablicht; bie

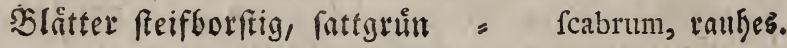

II. Die reifen Früd)te fteiftorftig

I bie slåtter 4 fad), brevnervig

a enfirmig, fteifborftig gefrangt (Wobr. fumpfige $23 i e(e n .23$ lbj. Juni) - rotundifolium,

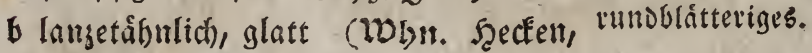

2giefer. Gebr. Farberen) * = boreale, nord:

2 Die Blätter sfach, lanjetformig, am licter.

Ranbe und an ber fielformigen Mits telribbe ruddmårtb feinftadjlid)t; bie Rnoten jottig (WWhn. Gärten, Heder,

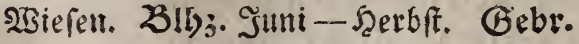

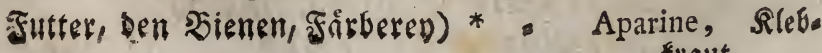
fraut.

Geni: 


\section{Genifta, Ssinfter.}

I Der Stengel gegliebert, gefligelt; bie Slätter lanjetåbnlid) evfơrmig (Blbj. Juni, Juli) - . . fagittalis, pfeilfor,

2 unbenebrt; bie slatter langetformig glatt; Die 2lefte aufred)t rundlid)t, geftreift (23Ib). Iuni-2lug.) = $=$, tinctoria, fátbes

3 bie Slắtter langetfórmig; bie unfrudhtbas ginftet. ren Iefte fadblid)t, bie bluibenden webr. IOB

a bie Şúlfen glatt (WWhn. Eaubbolf̧er. 2(1)3. May, Juni)* : = germanica, Deut.

b Die Şúlfen zottig (Bll)3. Spåtrom. mer)

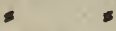 \\ Gentiana, Enzian.}

- anglica, englándt: pher.

1. Die Slumen 4fpaltig,

I ungebartet, fitiellog, in Quirin (woln. magerer Boben, Ojebirge. Zlbj. Iuni 2(ug.) *,

2 bie Slumenlappen am Ranbe gefrangt

(Won. uno \$ll);. mie oben) * " ciliata,gefianzter.

II. Die গlumen 4-5fpaltig, pråfentirteller. fórmiģ, am Schlunde gebartet (WWlsn. wie oben. 2 lbj. Aug. - Octb.) * = Amarella, bittes III. Die BLlumen 5ipaltig

cruciata, fteuf: formiget.

2 trichterfórmig

I ber Stengel gablicht, mebrblutthig (WDlnis. SBiefen, waldige Biegend,

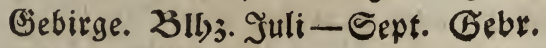

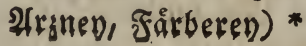

Centa urium, - Taulenoguloens traut. 
2 Det Etengel einfact);

a Die sisurgelblatter nieberliegend, gebåuft, grổert als die Etengel. blitter (WObu. feuchte sistefent, feltmer anf trodfnen. SIbj. Ipt. Shay, oft wieder im Sctb. Gebr. fie geben eine blaue Saftfarbe) * verna, Frúhling b bie 23 urgelblätter aufgerichtet, bach. enzinir.

ziegelformig, fleitter als die Sten= gelblatter (Whr. Bebirge) = bavarica; bairis

b prifentirtellerformig; bie seldhwintel lider. breitfluglidht; Die Silatter evfôrmig, ftiellos; Der Etengel vierfetitin (WOHI. S2Biefen. 2̇lb3. Stmi-Eept.) ${ }^{*}$ = utriculofa, baus c glocfenfiermig, idiget.

a långet als bet einfiad)e Stengel

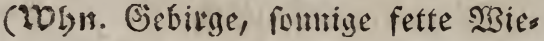

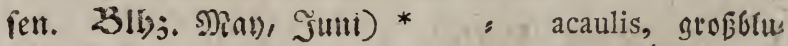

6 gegemiber, fo twie sie Blatter, migct.

I Inigeftielt; bie blatter enformig, lamjetăbulich, (d)arf zugefat (WVhar. (chattichte berge. Żll)j. 2lug. Sept.)* : " afclepiadea,

2 furbgeftielt; bie slatter ftiellos, f́l walbenwur

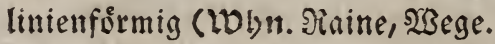
315). Juli-Sept.) * s Pneumonanthe, $c$ in Duirln; bie Sieldie abgeffumpft Eungenblume.

(20yn. Oebirge. Zlbj. 2lug.) purpurea, purputs d rabfermig, in Duinln; bie Sieldhe falei, rother. benfornig (WWhn. Siebirge. Sll)3. Juni, Juli. Gebr. Ju Drandtewein) 1 ut tea, getber. IV. Die Slumen 5-aud) Gfpaltig, glodien. fơrmig, umorbentlich punttit; - bie Selche

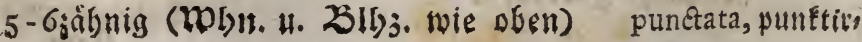
ter. 


\section{Geranium, Gtordfobutel.}

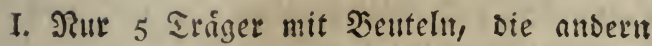
beutellos; bie \$lutfenftiele vielblutthig; bie Slåtter gefiebert; bie Şlåttchen jers fdinitten; Die Sticfe eingeid)nitten; bie Etengel nieberliegend (WWhn. auf Mecfern, an Straffer. Zlly. Ipr. - Sept.) * c i cu tarium,

II. Alle Irriger mit Seuteln;

(d) ierlingsblifteris ger.

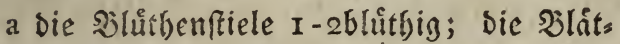
ter faft tellerformig, funftheilig, orey= fpaltig (SIl): Juni)

fangu ine u m, vilutwur.

b bie Slůthenficle 2blůthig

$5=$ Die \$lumenblätter aug̈getanbet,

I fo lange als ber fielch,

a verfebrt ber zfớrmig; bie S̉lats ter nierenfismia, 5-7ipaltig; bie Sticke 3ipaltig; bie Relche zugerpist; der Etengel nieber. geworfen (MOLn. Båute, Niaus exn, Straffen. 23!ly. StmiDetb. Gebr, furbie Edafe) * ro tu na ifolia,

$b$ bie Etempel ratili, troden; sie rundolátterigei. Slåtter jtbeilig, zimaltig; bie Bluthenftiele febr lang; bie 5arften burchgeljendo brifenlos (2引lyj. Epatfommer, 5̧erbit)

2 faft fo lang als ber Sieldi); bie diffectum, zets lifuittente. Siapreln uno gramnigen Sieldye flebrig, rauf); Die Slåtter nieren= fornig, faft 7 theilig; bie Theile 3 fwaltig; bie wegftebenden saare mit Drưfen

bohemicum, bớg: militser. 
3 bet Stengel aufredt;

a bie গ̧látter faft ráilofơrmig,

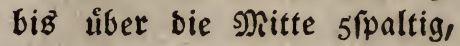
eingefdnitten (Whbu. Gebirge. Zlí);. Juli)

fy lvaticum,

$b$ bie Sluthenftiele uno bie ઝlüs thenblatter wed)felfeitig, bie Seapfelnglatt; bie Reldhe ftumpf (उll)3. Juli)

พBaloftortidinas bel.

molle, weidjer:

4 ber Stengel meitidneifig; bie Blåtter nierenforrmig, banbábn= Iid); bie Etticfe gleidjbreit, am Enbe gezånt; Die Reld)e gramnen. (DOB) *

- pufillum, fleiner.

[E. Die Ђlumenblåtter gam,

I rud wårts nervig, am Oirunbe sots tig; bie Slatter 5-7fpaltig; bie Stůce feilånnlid), langetformig, eingeidnitten

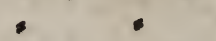

2 bie $\mathfrak{B l a ̊ t t e r ~ f a f t ~ f d h i l b f o ̛ r m i g , ~ v i e l = ~}$ theilig; bie Sbeile faft gefiebert, serfd)nitten, runglid)t, fpişig (Whir. Werge. Zlbj. Nanz 2 . Gebr. ben bienen)

pratenfe, Srsiefens

3 bie paluftre, Cumpf: fitordjidnabel. frotisidjnabel.

gerig; Die Slättchen gefiebert zer= (thnitten; bie Stúcke gerundet, mit einer Spişe; die Jieldue zebn= ecfig, bebaart (Wobn. an Strals fen, zwifhen Felien, auf Steins baufen, Mauernz. 23lbj. Manม.ug.) * 


\section{Geum, Senedictentutrs.}

I Die Blithen geneigt, ober liberbangent;

Die Grannen ber Fruidte feberartig

a gemunben bie Frrid)te länglicht (WWln. feuchte \$iefen, an Şrid)en. Zllyj. M(a) - Suli) *

6 gerade; Der Stengel rbluthig (Whin.

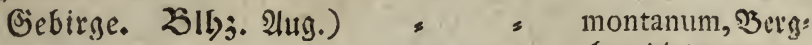

2 bie ஒlutthen aufrecht; bie Fruid)te fugel, benedictentwurg. benesictentury. forrmig, mit nackten, backenfírnigen Birans nen; bie Slåtter leverförmig (Whir.

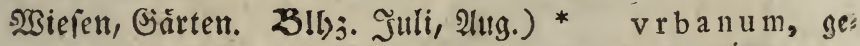

(Gebr. bie erfte und lejte $2 \mathfrak{r t}$, viel: meine.

leicht audh bie jwote, jur fobgarre, ben sienen geben fie auch Stoff.)

\section{Gladiolus, ๔dinertel.}

Die Slätter begenfórnig; bie જlưthen ein= feitig, entfernt (WDlyn. 23iefen. Bllyj. Sulli) *

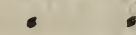

com mu is, go: meinet.

Glecoma, (Jundermann.

Die Slåtter nierenfơrmig, gefetbt (WDLn. Girtenlanb, an Gebantben, Båmten $2 c$. 2కlb). Frufling. Gebr. Futter, Den ミies nen, unter $\mathfrak{b i e r )}$ *

hederacea, ge: meine:.

\section{Globularia, Siggelblume.}

I Der Stengel mit vielen langetfórmigen Şlittern; bie Sisurgelblitter geftielt, enfores mig, am Ende bremsåtnig (MLn. magerer, founiger 3oben. 25lb3. N(ay) *

vulgaris, ges meille. 
2 ber Stengel faft blattlog,

a einfad); bie $\mathfrak{b l a ̂ t t e r ~ g a n z ~ w e r f e f r t , ~ l a n : ~}$ zetformig (LVhrt. Sod)gebirge. Zlbj. Juni, Jufi)

b Die Blätter berzåanlich feilfơrmig, am nudicaulis, naffi: ftenglicjte.

Ende eingefdnittell, Dreysånnig (WObr. u. ZIl)j. wie ben ber erften) * " cordifolia, bet:

\section{Gnaphalium, Siainblume.} blátterige.

I. Der Stengel ftraud)artig, offig; bie 2lefte ruthenfơrmig; bie Şlåtter fdimal, lanzeto formig, ftumpf, allentbalben filsig; ber Strauß zufammengeiest; bie Relchichuppen gologelb, (WVhn. an Felbwegen, auf Siat, nett 2c. Zlb3. 2lig. Sept.) "Stoechas, ges

II. Der Stengel frautartig, wie bießlåtter filgrig, meine.

I oben åftig; ber Sluthenftrauß suram= mengerest ; bie Silåtter wedbfelfeitig, Iônglicht, langetfórntig, fpizig, unten fils

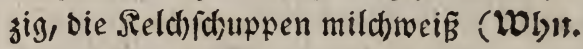
fette $233 i e(e n .23$ lly. 2lug.)

margaritaceum, petínfồtrige.

2 gang einfact);

a ber Slútbenftrauß einfach), am (Enbe;

Die Slutthen zmenhåufig; die ranfen nieberliegend (Whn. magere Plåke.

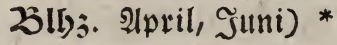

b Der Slůtbenftrauß̧ zufanmengefest; dioicum, zweys biaffige. Die Slätter lanjetformig, bie untern ftumpf; bieReld)id)uppen id)wefelgelb arenarium, Gands

c bie Bluttben an ben Enden fugelförmig rainblume. gebåuft ; Die Blátter linienfơrmig, lan: zetålnnlid), balbumfaffend; bie Rield )= fchuppen gruin, mit breitem, meiß̧en

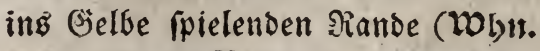
2derraine. 2ె!j. Juli-Sept.) 1 uteo a $1 \mathrm{bum}$, bleichgelbe. 
d bie Shlitfent aus ben obern Shlatt. winfeln, alt ben Seiten bes Sten. gels, in Sưfdelit, und einfact)

a furzgeftielt, werfebut fegelfürs nig; bie Epitenen ber Relcts fduppen braun (WOLn. Nabels bólzer. 23lbj. wie oben. Gebr. A( $\mathfrak{g}$ tiet) $)^{*}$

b ftiellog, fleiner, enformig; die fylvaticum, Şlåtter linienfơrnig; die $2 \mathfrak{3 u t : ~}$ gelblåtter fohnal langetfórmig, geftielt (WDIt. SiBaloungen. 23l5). Alug. )

Gratiola, đjottesgnabe. พืalorninblume.

germanicum, Deutidise,

Die Blüthen geftielt; Die Blintter lanjetfór's mig, fágegribnig (WDLn. Diebreiben.

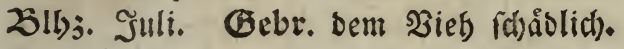
I(traney)

\section{Grimmia, S3rimmie.}

officinalis, milses 2fllin.

Die Rapfeln febr furzftielig,

I all Den Enden; Die Blâtter in ein Şaar auslaufeno; Der Deckel uno bie Bånge ser Sapfelmuinoung roth) (WOLn. Fels fen, Saum ftåmme, sie Siapfeln reifen im (Epithertfe) * = = = apocarpa, rotts

2 ber Dedfel gewolbt, jugerpist; bie Slåts Esprige.

ter angebriclit, aufrecht, lawgetformig, baarlos

a enformig (WWhr. auf Stcinen, Saumrinben) * = = ovata, evformige.

6 lánglid)t, verfegrt eyfôrmig, 4mal länger, als breit (Wobn. an ડ̉ăhs men) * lidjte. 


\section{Gymnortomum, Safinund.}

I Die Ståmmdhen aufrecht, affig; bie Blâts ter pfriemenformig, in trofnen Zuftande locfig, gerollt (Bobnt. auf Steinen, in

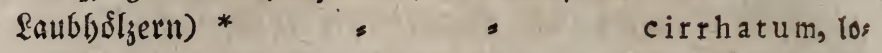

2 bie 3weige aufredtt, rafenbilbent; bie ckiger. Slatter langetfirmig, in ein langes fraar

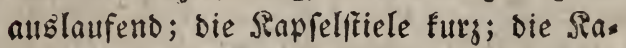
ffeln aufred)t (WDIn. Schindelbid)er, alte 5aumfrámme) *

3 bie Slatter flach verbreitet, eyformig, forarf gugefpigt; Die Siapfel verfebrt enformig, abgeftust; Der Deckel mit einer geboges nen, fd)nabelformigen Spiţe (Wobr. ůbers

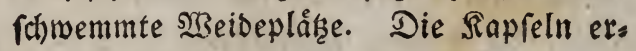
(cheimen im Miår , April)

4 bie Rapfeln aufrecht, birnformig; ber Des truncatum, abges fruztet. cfel jugerpigt; bie Epise etwas gebogen; Die Rappe pfriemenfórmig; bie Slătter enformig, burchicheinig (WDbn. Ebonboden, wo furges (Jras. Rerfh. im Şerbfte)

5 ftengelfos; bie Sapfeln aufrectst, gerwolbt, canefcens, grauer. lang zugerpizt; bie Epitze fohief; bie släts ter enformig, mit einem Sraar am Ende

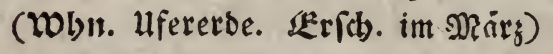

6 eiftig; bie Serpfelt aus bent Enben unb pyriforme, bitits fortmiget. Der Eeite, faft ftiellos; die Blåtter langets fơrmig, mit weifer butrdy)einiger Spikze; Die Selcblåtter mit langen Şaarfranzen am Ende (Whn. Steine in $\mathfrak{W}_{3}$ aldungen)

\section{Gypfophila, Sinpsfraut.}

ovatum, eyfor: miget.

Die Slåtter linienfơrnig,

I lamjetribnlich) 
a faftig; Der Etengel niebergerworfell, wurgeltreibent; bie 3weige aufredt) (S1): Jutui-21ug.) * repens, frie.

b flach; ber Stengel gablicht; bie Slumenblätter auşgeranbet

a bie Sieldhe fduppenlos ; sie ふlư thenftiele einblutbig (WWlnt. an

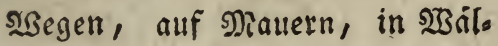
bern 2c. (Bl5;. Juli-Dct.) * muralis, Jianevs $\checkmark$ bie $\preccurlyeq$ luthenftele zmenblutbig (IVhit.?) - rigida, fieifes.

2 bie Sielche ectig, mit 4 eyformigen, zh. gefpizten Ed)uppen geftüt (IOb) গ্Sals

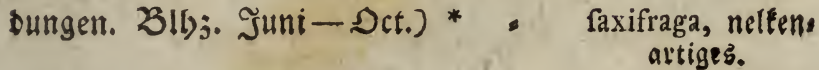

\section{$\mathrm{H}$.}

Hedera, Epheu, Winterguin.

Die Slåtter bis̀meilen enformig, unb gamg, bismeilen berzâfnlich, lanzetfơrmig, bis, weilen 3-5lappig (WDbu. 23nlbungen,

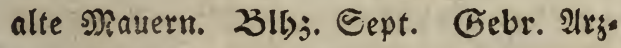
ney, Serberen, bie Secren zum 20 gel fang, bas hol' jum șein filtriren) * Helix, genteines.

\section{Hedyfarum, Şahnenfopf.}

Die Slåtter geficbert; bie Sullen glatt, ban= genb, in Trauben; Der Etengel aufred)t; ber Seld bebaart (WDIn. Allpen. Sll)j. Juli)

$$
\text { Helianthus, Sonnenblume. }
$$

alpinum, Milpens bahnentopf.

Die Slâtter alle berzformig, orennervig; bie Slůtben ůberbangeno (WOLn. wirb allent. balben gebaut. Bebr. treftichez Del, Schweinfutter, Den \$ienen, Frirberey) * annuts, getmeine. 
Helleborus, Niefsurts.

I Der Schaft einblutbig, faft naft; bie Slats

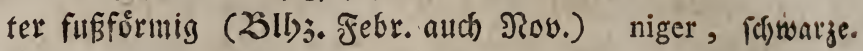

2 Der Stengel vielbluttbig, blåtterig; bie Blatter gefingert, fharf făgez̧ånnig (WDlyit. Giebirge. Zlyj. Nay, Jutmi. Gebr. Den Sieneit) $=3, "$, viridis, gruine.

Hemerocallis, รagblume.

I Mit fitwefelgelben \$lumen ( 5 lb;. Juli; 2inguft)

2 mit rotbgelben Blumen
Hepatica, Seberblume.

Die Slatter brenlappig; bie Rappen volffont: men gans (Wolnt. Raubholjer. Zllyj. Fes bruar-May. Gebr. Arzmen, Detr Sies neir) * . nobilis, genteitte:

\section{Heracleum, כ̉årentlau.}

Die Slättchen gefiebert eingerdutten; bie Sluetben alle gleid) (WObn. Wiefen. Zlb)j. Suni-Sept. Gebr. als jung 2iebfutter,

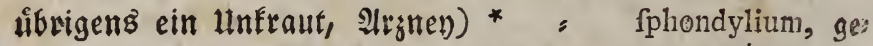
meinet.

Heriteria, Seriterie.

Die cingige 2 at wohnt allenthalben auf Ges birgen ober boben Gegenten (Blb;. Iuni, エuli)* ${ }^{*}=$ c a lyculatum, Herniaria, Soutfiraut. getbe 2rffodillilge. otaniengelbe. 
Hefperis, গiachtoeil.

I Die Stengeloli̊tter långlid)t, enforrmig, fpiß̨ig, vorfpringens, gezåant

2 bie Etengelblätter långlid)t berfformig, al= matronalis, ges meinet.

lentbalben gezăhnt; bie Slumenblátter ftumpf, am Enbe gans " " inodora, getuds)

(20hn. benbe auf Biebirgen. Zly;. Iuni, গuli)

\section{Hieracium, Şabidftfraut.}

A. Der Echaft armblatterig

\section{Ibluthig,}

a Init einem Kleinen Sblatte; bet Sield) mit weid)en Borften befest; bie STHurs

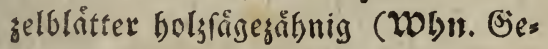
birge. $2 \mathfrak{3}(5)$. Juli)

b gutweilen beblâttert; bie Sllâttet lângs aureum, solbfars licht, gans, wollig bebaart; ber Feld febr gottig (Whan. Gebirge. 21 l)j. Suli, 2tug.)

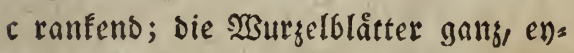
benes. lojer.

formig, bebaart (WWhn. 2 Sie Der. $\mathfrak{Z}$ ll)j. Jumi, Juli ${ }^{*}=$ pilofella, fleines.

b mebrblutthig,

\section{a ranfens}

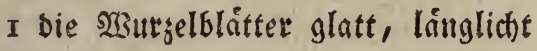
enforunig, mit Eleinen berworfprim genben Bâfuren (WDLn. Şecken,

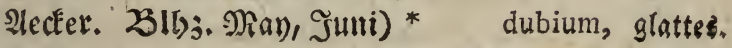

2 bie నSurzelblítter bebaart,

aa zutrgenfo̊rmig zugefpizt, voll= fommen ganzi felten gezånnt, (BObn. magerer Boben, Ruinen

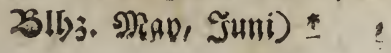

Auricula, grofés. 
66 evförmig, tiefgegrignt, nieber.

- liegent ; Das eimzelme Stenge!s blatt fleituce (Woln. Tonnige Dr:

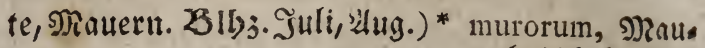

cc fotmal, langetformig, faft unges erbabiditftraut.

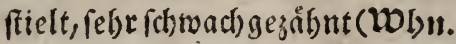

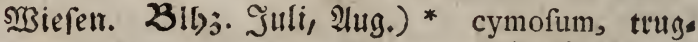

3 bie 23 urgelolitter faft tellerfôrmig, ooloenformiges.

in ber Jitgend fare bebaart, im Ilter glatt; Das eingelite Stengels blatt geftielt; bie SBlattfitele mols lig (WWln. 25 sloungen. 25ll);. Jult Szonig, wie bie 2 vorfergebenden 2trten)

4 Die 23 urzelblátter parfan gegafint p i 1 o fiffi in u m, langbaariger.

aa enformig; Die Blüthen fehr flein, an Der Epitze Des Eten. gels in einer Trugbolde; Die Relche mit einem ?ebenfeldhe, fil gig (Whur. Felfen. 23ly)3.2ug.) praemorfum, abs b6 linglidt); bie Slütben in eis gebiffenes.

ner engen Traube; bie Selde mit einem ఇebenf́eldie; bie Reld)id)uppen glatt ( 20 bru. Fel, (etr. 25lb). Juni)

lactucaceum, lat.

ec eyrutto lanjetålunlid), (h)wary. tiidjaltigez.

punctirt; bie \$lüthenftiele ein. blütbig, faft in einem Strauke; Die Slumen febre fadon fattrotb, ober oraniengelb; Der Reld (f)marzbarig; Der seiel jeder Sd)uppe am meiften befaart (Wolnn. Sebirge. Z3llyj. JuniEept.) 
B. Der Schaft mefrblitterig,

a âfitig,

a bie sluthenftiele einblutthig; bie SIlatter långlict)tenfơrmig, aufred)t, raub (Toln. magerer Soden. Zlbj. Jumi

- Die \$̧lưthenfiele mebrblúthig; die maculatum, ges
flecftes. 2Wurgelblitter enformig, lanjetäbn= lid); alle Sllatter gefitielet (WDbn. 23alounger. Blb;. Juli-2lug.) fylvaticum, $25 n$ ro:

c bie Etengelblitter fparfam, bersfor: babiditflaut. nig, umfafteno; Die Misurzelblatter gefticlt, långlicht enfờmig, gefriģt, gan

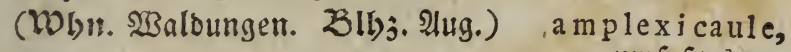

D bie ß̉åtter faft ungeftielt, evfơrnig, umfásteร. lanjetrínnlidy, fparfam, fein, uno jus weilen faft unfenntlid) gejåhnt; Die Saamen roth (Woln. allentbalben. 21t)3. 2ug. Sept. Gebr. Wachs unb f̧onig) * , = , fabaudum, fas

e Die Slâtter grob gejå̆bnt; bie obern vovifaber.

faft fponoonformig, umfaftend; bie untern gepitielet; Die Slütbenblätter uno Sielchichuppen fdiwars jottig

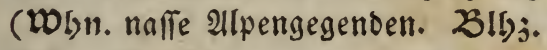
Juli-Eept:) = " : palu d of u m, f bie Sluthen bennabe in Dolben; bie Eumpfbabifits

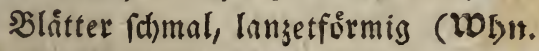
allentbalben. 23lbj. IHiguft, Sept. Gebr. bie shlutthen bienen ben Sienen, bie Blatter ben Echwei. nen; Das ganje Rraut giebt gelbe garbe)*

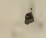

umbellatum, Dols senformiges.

b ein. 
b einfands

a bie Blluthen langyeftielt; die Blatter fefie fdumal langetformig, faft volltoms nten glattraltoig (Whan. Ilpen. Zllbj. Sommer) = " " porrifolium,

b bie Shluthenåfe fparfan, oben; bie land)bläteriges. șurgelblâtter id)mal lanjetformig; an Şlattfiele berablaufeno; Die Etengelblåtter gefietert zerid)nittent, in eine fămåd)tige Epiz̧e sulaufend; Die Stúcke zafulog, ungleid), gebo: gent; Det Sield) fatwats jottig (WWhr. Gebirge. 23lb3. 2huguti) = chondrilloides,

c bie sisurselblatter gefiedert serid)nits telt, an Slattfitele Gerablaufeno; bie thonorillenat: tiges. Stricfe baben am Enbe eine furze, fabenfơumige Spitie ; Stengel, Slät. ter und Aefte raub; ber Reld) lange banrig (Moln. Gebirge. Sll)j. Iuli, 2luguft)

humile, niedriges.

s bie Stengelblåtter hersformig, lans jetíl)nlid), umfaffend, gezaihnt; Der Rield) fel)r lofe, fanwar's jottig (Wotr. Giebirge. (25!b). Juni, Juli) - auftriacum, sfter:

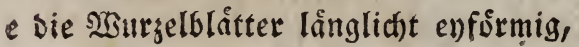
ieidsifides.

gerumbet; bie Stengelblätter balb. ummfaffend, långlicht; bie oberifen bers: formig; der Stengel ant Srunbe zwiid)en ben Slâttern zottig (WDbur. (jebirge. Zెlbj. 2ug. Sept.) = cerinthoides, f bie untern ఏlåtter geftielt, bie obern Immfafiend; alle evformig, lanzet. (ii) ábnlid), feingottig ; (Eine Sield)ichuppe entfernter (Woln. Serge. Zllbj. รัuli, \&(นguft)

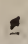
เwad) grbumenáfn.

vill ofum, zot: tiges. 
g bie Slatter umfaffend, zablreid)et als ben Der năchft vorgehenden airt, nackticheinent, in Der Ihat aber et: was filsig; die Sield)frufe faft gleich

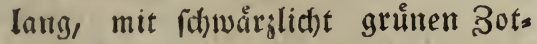
ten (WObn. und Blhj. wie bev ber vorbergebenden Art)

aurimontanum, IPondonblát:

Hippocrepis, Jferdef)uffraut. tertiges.

Die Slluthen geftielet, in einer Dolde; bie Şưls fen an $\mathfrak{A}$ Eenrande auBgerdineift (WWlnn. 23iefen, Saseiden. Wlb3. S)iay, Juni) * comora, Dolbens

Hippophae, Ðferoborn. thingender.

Die Şlåtter f(d)mal, langetfơrmig ( $\mathfrak{W}$ bnt. in 2uten an flůfen. 23lbj. Fruibling. Gebr. zu frecten, fodert aber fiefigen Boben) * rhamnoides, weir

Hippuris, Şippuris. Denblátteriger.

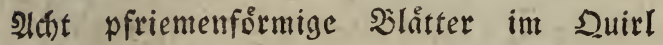

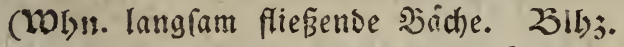

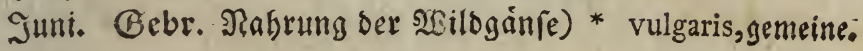

Holcus, Wferdegras.

a Die Reldre smenblittbig

I bas mainnliche Sbluttydjen unberwehrt; Des awitterblithdhens Girame fo lang als bie Spelze, ungegliedert, bafenfors mig (Whn. SSiefen, Inger, Feloraine. 25!h: Juni, Juli. Gebr. gutes Jut= ter) * $=$ = = lanatus, wolliges.

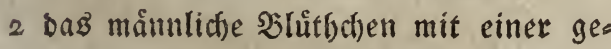
rabell, liber Den Seld) weit bervorras genten Grame; Die Bwitterbluthdhen unbewebrt; die 2lebrchen faft glatt (Whyi, Slbj, H. Gebr. mie oben) mollis, weidfer. 
b sie Seldhe 3blúthig; sie Seitenblithd)et

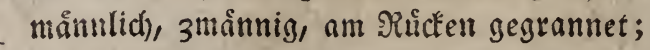
Das Nittelólưthchen ein Siwitter, 2månnig, granmenlos

\section{Holofteum, Epurre.}

odoratus, wofk riediender.

Die Shlüthen in einer Dolbe (MOhn. Idter=

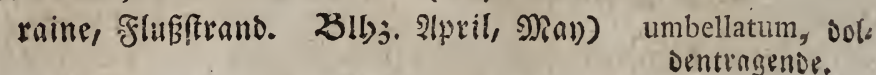

\section{Hordeum, bierfte.}

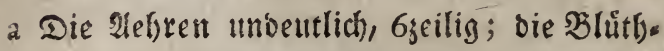
d)en alle frudtbar; bie Sanmell mit ber Epelge bededit (Wonn. allentbalben ges baut. 21bj. Jumi. Oebr. Sier, Brod,

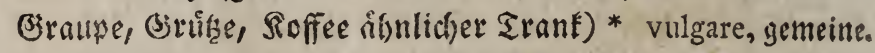

b Die Shlitbchen in swo Beilen frudtbar, it ben viet zimifhenliegenden unfrudtbar (Wobn. feltener gebalt. 25lbj. H. Gebr. wie obeli)

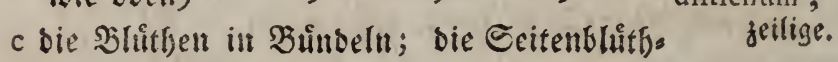
d)en monunlict)

I alle mit Eirannen; bes S̉lutthdenbins Dels Umidhlag 6grannig; die mittlern Giramnen aus bem Birunde (DOLn. Sduttbaufen, an 5̧åntern. 25lbj. Jimii) * " = murinum, Måaץes

2 Die Granne bes Sittelblithden febr lang; die Umfalläge franzenlos (WDyn. (S)ebirggegenden. 23/bj. Juni) *

Hottonia, Sottonie. gerfte.

montanum, $\mathfrak{b l g :}$ gerffe.

3ablreid)e Sllithen in Ouirlen; bie જląt= ter it Quirlen, gefiedert; bie Stưde linienformig (WDln. ftebendes 2153. Juni, Juli. Gebf. Den Bienen) paluftris, Eumpf: bottonic. 


\section{Humulus, 5̧opfert.}

Der sabme Sropfen if wom nuilden im Grumbe nidht werichieben; nur mad)t ifhn bie Siul.

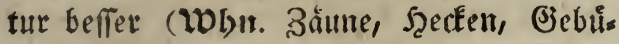
(che, Girirten. 2Bll)j. Juni, Juli. Gebr. Die $\mathfrak{S B M}_{3}$;elfiprofien als Salat; die Zapfen

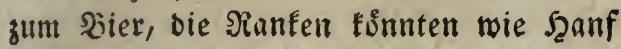
bearbeitet und benúk̨̧t merben)* . Lupulus, gemeiv

Hyacinthus, Şyacinth. ner.

a Die Blunten edig malsenfsrnig; bie obern unfrud)tbar, fleiner, länger geftielt ( $\mathfrak{W}$ b) 25iefen. 23lly3. Mian) $=$ " comofus, gezopfs

b Die Şlunten fugeltund, gleidffơrmig; die Silâtter wạlzenfórmig, mit einer アRinnê an

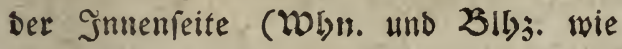
oben)"

c bie Slumen enrund, alle gleidffórmig *

d bie Silumen tridterfórmig, balb Gipaltig, am (sirunbe baud)ig

e bie Şlumen 6theilig, an ber Epiz̨e zurưcts

botryoides, Traus benfyacintb.

Mufcari, Miufcas tenfyacintt.

orientalis, gemeis gerollt

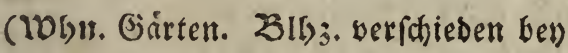
non fcriptus, nies Den 3 lestern 2rten.)

\section{Hydnum, Stad)elpils.}

2. Der Strunf fefr furz, am obern Ende

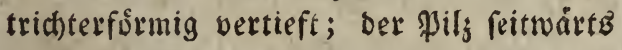
auffitieno, boljig, weißj, oben mit concen. trifchen Sireifen (WWbn. alte Saumftooffe) * p a r a fi t i c u m,

b ber Strunt febr dick, verfenft; ber \$il, lidmarozender. febre âftig; Die 2lefte und. 2leftchen mit viels åftigen weichen Etacheln allenthalben bes feşt (DObit. auf faulendem Fosbrenbolge. (1Erid. im Sept.)*

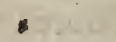

ft a la et i ticum, tropffteinartiget. 


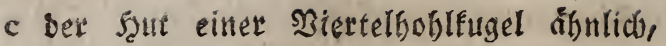

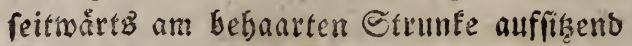
(Wohn. argefalleme Fidjtenzapien. Kerfid). in herbfte) * 3 :

d ser Satt grofi, gefduppt, in ber Mitte ver. aurifcalpium, ofr: lofferformiget. tieft; Der Strunt bicht, waljenformig (Whi. Э?abelfolzer. (Ėrfd). Sept.)

e ber Sait gewolbt, glatt; 'sie fiad)lichte llts imbricatum, ge: libupter. terietite etras am Strunte berablaufend; bie Etacheln feitwaits frehend (Whu. Fidjtenmurgeln. sizrd . Sept.) * s repandum, ges f bet \$sils bals geftielt, balb ungeftielt, feine (id)rocifter.

Confifeny, wie die eines balbgefottenen (Enweifies; die Stacheln enmeiffärbig

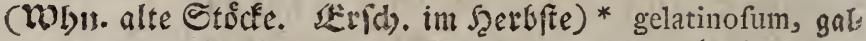
Hydrocharis, Srufabifí. lertiget.

Die cingige befannte 21tt wolsut in Giras

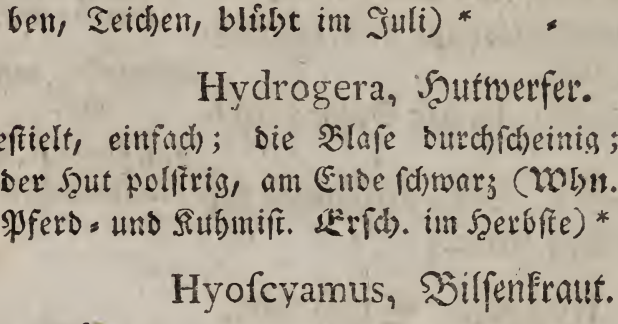
Morfus ranae, ges meinet.

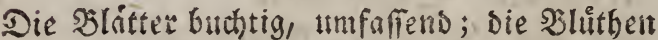
fitellos (Whys. an Etrafien, an Shiufern.

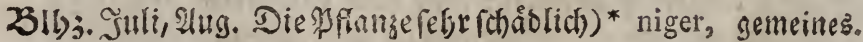

Hypericum, Johannisteraut.

Die blüthen 3reibig.

2. Der Etengel 4fantig, frautartig; bie Şlus menblitter am Sande mit (d)warsen Punfts oruifen (Woin. SBiefen. 25!bj. Juli. Gebr. Den Bienen) *

cryftallina, fros frallibeller. 
b ber Etengel jwebldineibig,

I fabenfirmig, meiffdweifin; bie şlithen

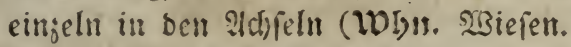
25lb). Julit)

2 bie blumenblätter fownars geftreift und

humifufum, nies Dergetworfertes. punftirt; sie Slatter eyformig, burch) fdeinig punttrt (Wobr. ISiefen, Iledfer. 2153. Iuni, Juli. Gebr. Färberey) * perforatum, ges

e bie Sreldhe fägęånnig, sruifig, Der Stengel meines.

walzenruns

I glatt; Die Slåtter ftiellog, umfaffend,

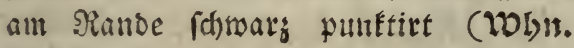

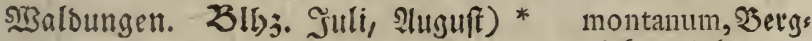

2 rattb; bie Slâtter långlicht eyfo̊rmig, bebaart ( $25 ! 53$. wie oben) jobnnniskraut.

hirfutum, zottis Bes.

\section{Hypnum, 2lfmoog.}

I. Die Sluethen Der mo̊nnlidben \$flange Eno: fpenfortmig;

I bie Etånmd)en mit alweigen fact) ge: fiebert; bie 3weige einfad), unter lech) ten 23 infeln wegftebent, am Enoe bes Crammes eine Ilebre bilbend; Die જląt. ter bachjiegelformig, fichelåthilid) (WOLn. 2orbơlzer. Zieife April)

filicinum, facten: Fiautíhnlitges.

$\approx$ bie Ctåmnte nieberliegend, Surd)aus stuevjeilig, n̊ftig; bie Aefte mit 3weis gen gefiebert; bie Slatter angebruckt; bie Saprelftiele aus ber Jitte Der Zwei= ge und $b_{C B}$ frautftammeg, einzeln und gebåuft (Whit. Ş̧aldungen. Reife Frubling) SBnndaftmoos. 
3 bie Ctåmme in LÁntfernungen wechfels. weife âftig gefiebert; bie ßlátter gerabe; bie Frudtftiele gebånft (WWhn. U. Keife wie oben)

4 bie Stămme faft mit 3meigen gefiebert, proliferum, iptofs fendes.

aufieteno; bie Zweige an Der Spize abmînt gefrummt; Die Sllitter lanjet. fơrmig, febr fa)arfipingig, abwoirts fichels formig gebogen (WDln. wie oben)

Crifta caftrenfis,

5 bie 2 efte zerftreut; bie Slatter brenccfig, fpitigig, nervenlog, im trodinen suftande geftreift, unordentlid) wegftehend

6 bie 2lefte faft nieberliegend; bie 3reige federbufdábn liches.

triquetrum, bievs ect์ โ⿳亠ttterigez.

unorbentlich) zerfiteut, runblicht, glån= geno; bie Slätter lơffelfórmig, lanzets signlid), gefpiţt, ober mit einem furgen Şaare geendet, faft nervenlos, bactsies gelformig (WWhtr. feudte Girůnde) illecebrum, Ealis

7 bie Ctỏnme âftig; bie Aefte weitlåuf. attiges.

tig, faft zwenfeitig zmeigig; bie ameige runblicht,

a nabelfórmig; bie Blâtter länglicht, nervenlos, bie Des थft, EnteB in eine fteife Epitze übereinander ge.

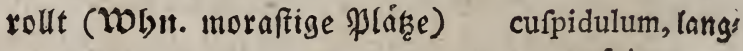

6. pfriemenfórmig, etwas abwoirts gefpiztę. gebogen; bie Slätter lanzetfor. mig, mit einer Nittelribbe; bie Enden ber Iefte nidht fpişig, nicht fteif (Wolnt. feudbte (jebuifhe) inerme, weble 8 bie Ståmme mit rundlichten 3meigen un= ไoโeร.

orbentlid) gefiedert, glånzeno; bie Slät ter loffelformig, in eine furge Granne

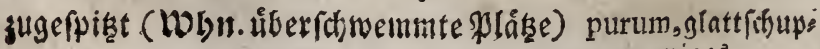
piges. 
9 bie Ctåmme niebergenorfen; Die Nefte aufred)t, etwas stweigig; Die Epizen ber Ilefte tiberfangeno; bie Sloitter umfalfent, breitlid)t, fpisig lonjetformig, geftreift; Die Rapfelftiele cingeln; bie

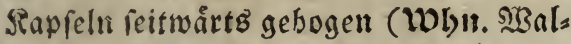
Dungen allentbalben. Die Sapfeln int (ommer) s - - fquarrofum, fitrat:

II, Die Bláthen ber mainnlid)en SPFange viger. fuopfförniz;

I bie Strimme nieberliegent, mit 2ieften Dopwelt gefiedert; bie Aeftchen sweigig gefiebert; bie Zweige fehr flein; bie Silátter Dachsiegelförmig, faum fid)tbat ('DI) SYGalbungen)

delicatulum, fat: tes.

2 bie Stămndhen dufrecht, mit einent sisurgelfilse, oben mit gafleciden, parals Ielen, einfachen 3weigen won veridbie, Denem (Beftolectite; bie feud)ten Blâtter

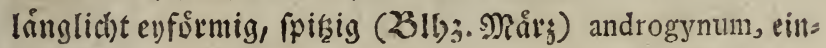

3 bie 3weige aufrecht, waljenformig; bie bộfiges.

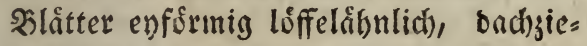
gelformig, angebricft; bie Raprelftiele aus bem Sirunde ber Strimund)en; bie Rapfeln iberbangend (WWlyn. feuchte

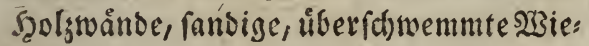
ien)

,

argenteum, filfick: Jlótteriges.

JII. Die Blúthen bet månnlidjen Pflange fternformig;

A. Die Rapfelt geftirist,

I walgenfơrmig; Der Deckel Eegelfor: mis, furg; die Silitter langetiolunig, mit einer baaribnlidjen Granne (Whit. Feldraine)

capillare, baaris geל. 


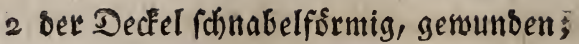
bie $\mathfrak{B}$ låtter enfơrmig, mit ciner Bors ftenrpitse; bie Ståmmoben oben bin. Delforrmig, zweigig; bie aroeige ull. ten fiemlid) Eabl, oben blåtterig (WDitr. 23 aldounger)

annotinum, meis

3. Die §affeln ůberbangend; Die Etåmm. Denblatteligez.

d)en einfach

2 bie frudstbaren aufrect), bie unfrudbts baren nieberliegend; bie Slâtter burd,icheinig punftirt, mis einer Mittelribbe

a volffommen gang, verfebrt enfoers mig (WObn. Steine an WSaffern

b figegánnig, gleidhlang, langetfór:

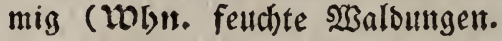
Zeife Jrůbling)

$b$ alle alffred)t;

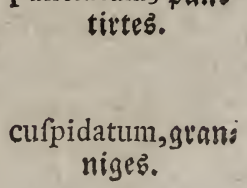
tirtes.

I Sie Spitze rofenfơrmig blåtterig; bie Blatter nid)t fo gleichlang, werben im Stodfnen nicht fo wel. Ienformig wie bey ber nåd)ftoors

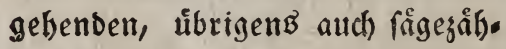
nig, langetfórmig, punftirt (Woln. wie obett)

$=$

rofeum, vofenfor: miges.

2 unten fafi nackt; bie \$latter långs lidjt, mit einer baarfórmigen

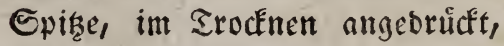
mit wegftehenden Epişell; bie Blútbenftiele aus bem Grunde (Woln. Felonege, grober Eanb;

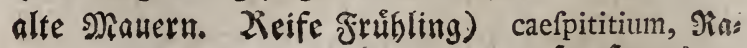
fenaftmoos.

C. bie 
C. Die Sapfeln etwas peitwarts geneigt; fugelformig, birnåtnlid); Der Dedfel flein, Eegelformig; bie 3weige fnum aftig, gans einfach, fabenformig aufs recht; bie Slátter angebrůcft, im feud)= tell Zuftallbe nad) Einer Eeite gerid)s tet (Tobn. an $\mathfrak{S a f f e r n ) ~} \quad$ = fontanum, Sucls

IV. Genvifie aftmoofe: nod unbefimmte nfrmoos. Jamilie.

A. Die Etrimme und Ilefe friedhend;

a bie 2efte mit Zweigen flad gefiebert; bie Zweigeblätter idjarf lanjetfirmig, ftammmárts einfeitig, fichelfsismig; Die Sapfeln ziemlić) aufrecht, evfôr = mig (Mobri. Sצaldungen)

compreffunl, zas fammgeovichtes.

b bie 3weige nufrecht,

I gerabe, ftumpf; Die Slátter it cine feine, itharfe, lange Spite aus: Inufens, gefteift, obne Mittels ribbe; sie Siapreloefel fury, fegel= formig (MOln. Salmutubcr. Die Siaffeln fommen in Scornung2(pril) $=$

-

rutabulum, fití

2 an ser Epite fich abwaitto Eruin. ấenformiges.

ment, wurjelno; bie Blatter nur fpişig, nicht geftreift, mit eimer Mittefrible; bie Rapfelbedel lang, fonabel. ober pfriemenfirmig rufciforme, murts

B. Die fruchtbaren Ståmme aufrect)t, mit thenblatterigez. uீberbangenten aweigen an ber Epire, bie unfruct)tbaren nieberliegent; bie Slåtter långlicht, gezáflut, im Irodf́nen gerwellt; bie Siaplelfiele aus der Spiţe, zablreid) (Woln. feudtite (jebuifdie)

undulatum, wels Ienformiges. 
C. bie 3weige öftig, malgenformig; bie ミlätter enfôrmig, nervenlog, punf́tirt, backsiegelformig (WObn. feuchte, fchats tige \$hitise)

V. Zmeifelfafte 2 ftmoofe.

lucens, glänjett: oes.

I Strimme und 2lefte friechent, fabenfors mig; bie 3weige aufrecht, gebringt; bie Sapfelftiele atts ben Grunde ter Bweige, etwas lánger als fie; bie Rapieln und Dectel gebogen (Wolnt. alte Saumrinben, faulende Dlnitter. Die Stapleln im 23 Binter)

2 Die Stånme friechend, bie 2lefte aufrect)t,

clavellatum, feut: fôrmigež.

a umorbentlid) mit 3weigen gefiebert; die trockinen Silatter fichelforrmig ges

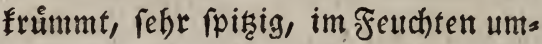
faffent evfơrmig, an ber Spisce ein. gefdnitten (WObn. Saummurgeln) cupreffiforme,

b faum zneigig, lang, unten bưmer, Suptefienafmoos. an ber Spire geftirnt; Die Sappels fiele aus ben Seiten ber Aefte; bie Blitter langetformig, siemlich lofe ftellatum, fietno

c zweigig, vielbluttbig; bie Zweige am formtiges. Ende jugeipint, etwas frumm; bie Blítter eyfórmig, fpişig; bie Rap: feln nufrecht, ibre Stiele aus ben Alefen (Whn. Baumrinben) = myurum, ungleis

o an bebden Seiten nicht zerfirent; bie c)eร.

Stomme etwas fiach gebrictit; bie Silitter in 2-3 Reifien, langlanjet. fơrmig, fipişig, burd)icheinig, queer: gewellt; Der Stamm unten mit bralts nen Zotten sid)t befleibet (Why. S3alsungen. Die Rapfeln fommen im ḩerbfte)

- undatum, getvelfs teร. 
3 bie Staimme aufred)t, unten gmeiglob, oben bunbelformig, sweigis; bie Bmeige runblidst, meifteng einfach, aufred)t; Die 23 urgel Eried)end, jottig (WOLn. Salsungen)

4 bie Etomme bis binauf mit einer bralts dendroides, baums nen $\mathfrak{S}_{3}$ Ulle befleibet; bie 3weige an ben Enden Dicflid)t; Die Siapfelfitele ge: munben; bie Slåtter lanzetåbnlid), fiel. formig, in ein Şaar geenbet (Whls.

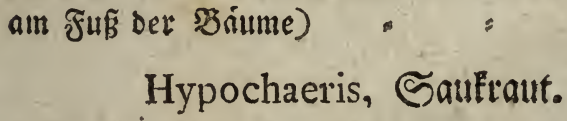

I Der Stengel meiftens Iblütbig; bie Blât: ter lánglid)t enfơrmig, ganz, gejấnt

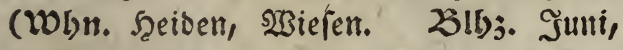
Juli. Gebr. ben Edweinen Futter, Den

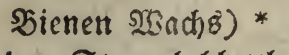

2 ber Etengel blattlos,

a neiftens einfach; bie Blithenftiele idjup. pig; bie SBurgelblatter nieberliegent, boljfigegágnig, frumpf, rath (2)lbs. Juli. Gebr. gefundeg গ3ichfutter) radicata; lang:

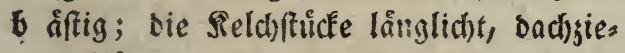
incantum, grates. artiges. belformig; bie Şantrone der Rand: blüthden fitellog, bie ser Mittelblitt): d)en geftielt; Die Slitter bud)tig, ges zábnt; bie \$flange glatt (WWbr. trodfne 2̧̧aldungen. 23ibj. Juli) - glabra, glattes.

\section{I.}

Iafione, Jafione.

Die eingig befannte $21 x t$ molnt auf Sorgen unb magern Wiefn allenthalben. 25lbj. Juni-Eept. Gebr. Den Dienen * montana, Şerg: iafione.

Iberis, 


\section{Iberis, Sautunfenf.}

I Die Blatter enfớntig, bie am Stengel umfafients, glatt, Dicflicht; bie Edfortthen prifmatifd); Der Griffel Thief (MOLn. Sies birge. Bl(t). Julli)

rotund if olia,

2 Die Slátter gefiedert gerfd)nitten; ber runoblítteriget. Stengel blattlog, vollfommen einfach (Woln. am Jfarftrande) * , s nudicaulis, naft. ftengliget.

\section{Ilex, Stectpalme.}

Die Shlatter enformig, fpifigig, am sianoe ftachlictit, ober volffommen gans (WDi)r. Biebirge. Gebr. Die Shlutben Den Sie.

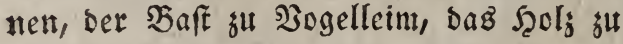
(c)ơnen Arbeiten, bie \$eeren eine Surtel. taubenfpeifie)

\section{Impatiens, Epringfraut.}

aquifolium, ges meine.

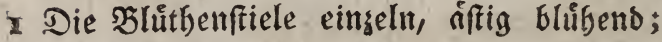
bie Blatter enforrmig; Der Etengel unter Den Slittern angefdrwollen (WDLn. fchats tige, feuchte sisaldungen, an ß̉åchen. Zlbj. 2lug. Sept.)*

2 bie Shlůthenfticle gebåuft, Iblüthis; bie Noli tangere, ges meinte. Slätter langetformig, bie obern wed)fels=

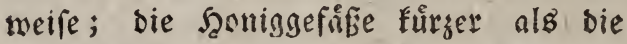

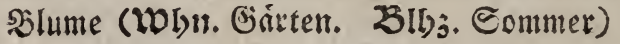

Balfamina, bals jaminen.

\section{Imperatoria, Meifterwur.}

Die cinsige befannte Ant wolynt auf bes birgen, blílgt im Suni unb Juli = Oftruthium, ges meine.

Inula, 2Ilant.

Die Slâtter umfaffen faft in allen Irten Den Stengel, 
2 fint entunt, runglidt, unten filsig; bie Reld)(t)uppen evrumb (WDLn. an Żåแ nen, Dôrfern, felten auf 2 Biefen. 2B!bj.

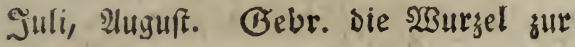
มี่แtev)
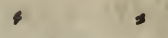

b find langlanzetformig, vollfommen ganz, uno fo, wie Der Etengel, allentbalben Dichthaarig; die Slutben meiftens ftrauf. formig (Whrr. Dưre Pläte. 25I5j. 2ug. (Ept.)

$$
\text { ? }
$$

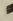

c find langetformig,

IIelenium, wonfs; let.

I fågezoifnig, unten zottig, ober glatt weiflicht grün; Det Etengel einfach, over siftig zottig, oder raub; Die Slutbenftiele an oer Spizze filsig, sblutbig (Wour. an Straffen. ZSIl)j. 2uguft)

2 glatt, - britannica, enga líndifficet.

Oculus, grofblus miget.

a feingefrangt; bie Aefte etruas ecfig, glatt (Monn. feud)te Wiefen uno

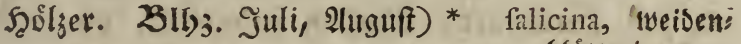

6 nervig; Der Stengel einblutbig (כIb). 2ug. Eept.) blätteriger.

enfifolia, Degens blátteriget.

3 etwas fteifborftig, farf bebaart; sie untern Blutben borber wad) fend; Der Stengel rundid)t bebart (20but. wie oben. 23lb5. 2(uguff) "hirta, vauber.

d find långlicht berzformig, etruas filfig; Der Erengel mollig, geripet; die felch. fdrppen borfenformig, filgig (MLIt. feudite Drte, an Grâber. 23!b3. Juli, 21!. Bebr. 2ranev, ben \$imen) * d yfenterica, Pubralant.

c fins 
e find wellenformig, bicht befaart; bie Slutthen faft fegelformig; Die Stralo blümchen febr furs (Wobn. Giråben. 23153. 21tig. Gebr. Der Rauch Davon veriagt Mitcfen und flơhe)

pulicaria, gisf. alant.

\section{Iris, Stwmerotlilie.}

A. Die Siarben ungebartet,

a grof; bie unfruchtbaren fleiner als bie Slumenblätter; bie blêtter (d)werd. formig (Woln. Siräben, Teiche. 23!l);. Suni. Gebr. Die ş3urgel zur Rof)= garre) * - . Pfeud-acorus,

b bie Slätter linienfơrmig;

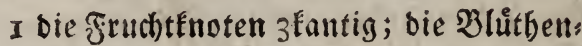
blatter vertrochtnet; ber Etengel runo, Ingefábr 3 blüthig (WDl)!. ŞSiefen. 25lh3. Juni) " =" fibirica, fibitifife.

2 bie Sruththnoten Gecfig; ber Etengel

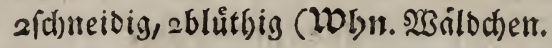
2lis3. Juli) " , graminea, grass

B. bie frud)tbaren నarben gebartet; die blåtterige. bartlofen volfommen gans; Der Etengel vielblůthig, långer als sie Blåtter; Die untern Sluetben geftielt (Woln. Sirirten, feuthte (jegenden. Zlbj. May. Gebr. zu Saftarben)* " $\quad$ germanica,

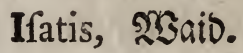
Deutiche.

Die Sisurgelolitter geferbt; bie Etengelblöts ter volftommen ganz, pfeilforrmig; bie Bblü: tbenftiele glatt; bie Edbettthen lămglidut (WDLn. auf cinem Jergablang. $2515 j$. Miab, Jimi. Gebr. Fãrberen)

- tinctoria, gemeis net.

Iuglans, 


\section{Iuglans, গֻּallnußi.}

Die Blatter beftehen aus fơrmigen, glat. teli, fatwach fágejábnigen, einander faft gleidgen Slättchen (Wobn. an Straffen, in Dorfgarten. 23ibj. Nav. Gebr. Die গuiffe seben eine gute @peife, gutes Del, gute Nild, bie balbreifen, werben einges rotten. Die grume Edjaale Dient zum Fărs ben, fo toie bie Rinde. Şols und গুM zel gu ben (d)ơnten Arbeiten) * " regia, gemeitre.

\section{Iuncus, Sinfe.}

I. 'Der f̧alm blattlog,

A. aufrecht;

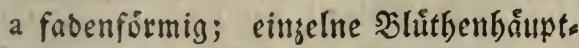
chen an ber Spitze megrblátterig; bie Ș!atter linienfơrmig, rinnenăbnlich

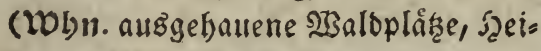
Den. 25!bj. Juli)

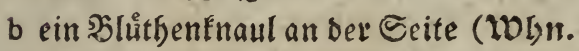

er icetorum, feeidebinie.

Siráben, Sưmpfe, Noråfte. 23!ly. Juni, Juli. Gebr. als jung zum হieffutter, bas Nart zu (lidttod)t, Die trocknen Etengel zu Strobtel. (erii) *

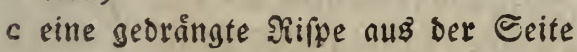
conglomeratus, sinopfoinfe.

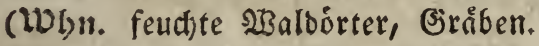
2̧153. Juni, Juli. Bebr. als jung zum Futter, fonft zu siorben uno $\mathfrak{T}_{\mathrm{a}}$ peten)

d eine rwieberfolt trugbolbenfơrmige effufus, Flattep binle. Siipe an ber Cpite; bie untern Riipenåfte långer, alle gebraingt, mit Doppelten Scheiden (WDl)n. naffe

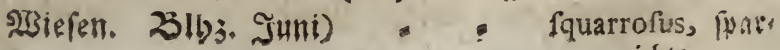
ridite.

B. et. 
B. etwas ůberhangens; bie SRippe aus Der

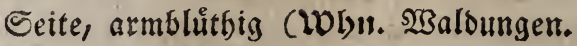
23lbj. Juli. Gebr. wie oben)

II. Der Salm beblåttert;

filiformis, fabens formige.

a bie Släter rofbrig, nach bent Erocfinent fnotig; Die Siifpe einfad); bie Reld): blätter mit einer furgen Granne; bie SJlütbenanfíbe fürser als bie Sllutben: båatptchen (WWht. Bebirge. Wlbj. Suni)

b bie Slåtter sulammgedricft, fnotig,

geniculatus, ges gliederte.

I you Der Rainge bes fralmes; bie SRifie armbleithia, aufred)t, viel fur:

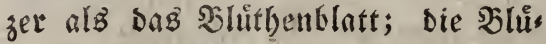
themanfritze langer als bie bluthen (Woln. Moosgruinde gwifhen Obebirs get1. 23153. Jum)

alpinus, 2f(pen:

2 ait Ente utro an Der Seite Der Sil binie. fpeniffe ftebcit bie 3 blütbigen frăupts d)en (WDhn. naffe Slákęe. Sll);. Juli)* = "

c Die Slâtter (d)wad)Enotig; Die Slutben tenteinie.

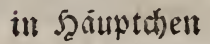

I au ben Epiz̧en unb Seiten ber åfti.

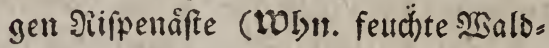
plóke. Slb;. Juni, Juli) - rubnodulofus,

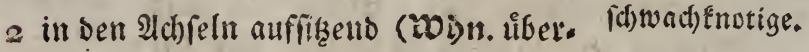
fdiwemmte Siebreiben. Żlb3. Juli) fafciculatus, bus y sie Slitter rinnenforrmig, liniettännlich); ifferbåuptige.

- Die silpe an ber Epitze, etwab zers fivent; Die Ieşten SBlüthenftield)en febr furr; bie Frud)t fugelfornig (WDLn. an (iráven. 25lbj. Juni)

bulbofus, thol. lige. 
e bie Sblitter borftenformig, ecfig; bie Şlưtyd)en länglicht, an Den Aefen ger: freut, ftiellog (WObur. feuchte \$läß̨e, in

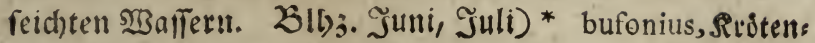
f bie Slätter pfrientenferrmig;

binie.

I ber f̧alm Iblütbig (Wobn. Giebirge. 23 (b). Tutui)

monanthos, ein.

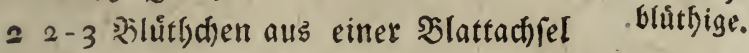

ant Ende bes Şalmes (WWhn. und

23it)3. wie oben)

trifidus, oveyblú:

thige.

g bie ஒblätter flach, behaart;

I bie Blůthenbunoel åftig, länger als bas früręende Slatt (Woln. Siachfels Der, şaloungen. Z3lb3. Frůgling) Nit 3 Abarten * = = pilofus, baatige.

2 bie Blưthenblátter långer alB bie

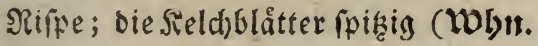
faubboflzer. 23lbj. Fribling) * niveus, toeibe.

3 mehrere 2lefrchen an Der Epitze, ge. ftielt, und fitellos (WOLn. Sistefen, Hecter, Waldungen. Bibj. MårzMiay) *

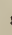

- campeftris, gelbs

Iungermannia, Jungermannie. binie.

I. Die Etengel nur mit Slättern gefiedert;

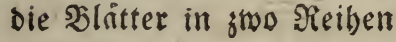

a volffommell ganj,

a (aud) fein gejåthnt) aufiwårts gerict. tet, faft tellerformig (WDlyus. Iaubs bolytwaloungen. Die SRapfeln ex: fdseinen im frrubling)

B Dadsiegelformig, vertieft (WDlno. tho. viticulofa, langs rantige. nige Aecfer. Die Slíthen im Frúf. ling)

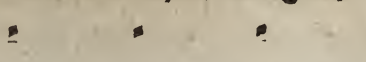

polyanthos, viels Glúthige. 
c Die Etengel lanjetformig, einfach), ober unter red)ten (Whur. fdattige Raubbólger) : lanceolata, lanjef:

b im Sroffuen unten vertieft, mit $u$ = ftenglige. endlid) Eleinen Änfăngen von Fran. zent; Die 3weige rumblid)t (?)

aquatica, Şaflet:

b gefrangt, faft tellerformig, wedjelfeitig jungermannie.

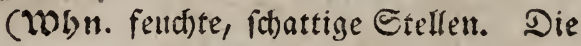
Rapfelt ifmen fich im December)

c am Eube feitjt aubgefdweift, jwevzåt) afplenoides, milfs frautartige. nig; sie âthne febr feit, langfpithig (WDir. unter ben Moofen)

bifurcata, aubges

d am Enoe 3-Gåannig, am Sirunde (a)mås (d) weifte. let (iolsw. wie oben)

quinquedentata, finfzólbnige.

II. Die Gtengel mit Bweigen, die 3weige mit ふláttern gefiedert. Sit ßlattol)ren.

a Die 3weige åfig; bie Silntter lôffels formig, Dadjiegelformig, gang; die Slattobren unten, wedffelfeitig, enfors nig (Whu. in Piaselforljern)

b bie Slâtter unregelmáfig vierecfig, am undulata, flaubٌ blitterige.

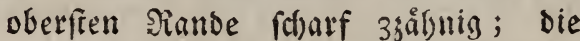

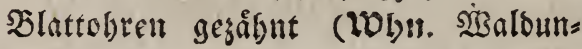
geil) *

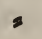

trilobata, orelle

c Die Sláter eyformig langetånnlich, an zoñnige.

ber Epitge feit 3jâfnig; die Epuble unten mit runolid)ten, entfornten Slatt: anfótan befest (Wont. feud)te Stels Ieit)

d Die Ståmme 4reifig mit refupinata, umges febite. fiebert, burchaus friechend; Die Sln̈tter 4jåknig (WDin. feuchte Dergwaloun. gen)

$x \in p \operatorname{tans}$, flies cjende.

III. Die 
III. Die Stengel badfsiegelfoirmig gefduppt;

a Die Blåtter in jwo Peifhen, balbteller. áfnlid); Die Zెweige an Der Spize fructttragend (Whan. Saumrinden)

$\mathrm{b}$ Die Slätter in vier Sieiben,

I bie tmtern amal fleiner (10in. Steine in Sorgwaldungen)

epiphlaea, $\mathfrak{3}$ mum: rinoenjungerman: nie.

tellerformig, oben genoflbt, unten tamarifci, tamas vertieft; Die Zmeige an ben Enven breiter; Die Epuble mit entfernten Şlattanfåzen (MObn. Soumrinden. frud)tjeit (fruffling) : . dilatata, augges

IV. Die 3meige runbum mit jerftrenten hant: breitete.

formigen, vieltbeiligen \$låttern geidhupht; bie 3weige rundlicht; Dab (ỉewåd) flein (WDin. wie oben)

trichophylla, baarblátterige.

V. Der Stamm feloft ein Blatt,

a linienformig, âfig; tie Enben gerunbet (DOhn. feuchte Scolsftamme, aud) unterm Moofe) . furcata, gabliffte.

b gablicht, bicklicht, fumpf, am SRlucken murzelnd (Woln. fandiser, feuchter Ibon)

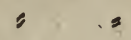
$=$ pinguis, fette.

Iuniperus, SGadjolder.

Die Slåtter brenfach, wegftebend, ftunipf breyfantig, febeno, långer als bie beere (Whln. Worbofiger, ausgelichtete $23 a$ albun. ger. Gebr. Die beeren zur WBurge, zum

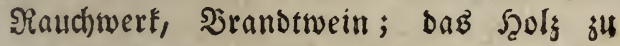
cingelegten und Dred)blerarbeiten, aud) in groffen Ståmmen z̆ Ébeniftenarbeiten; Das Sar giebt Eandaraf́) *

communis, gemeis ner. 


\section{K.}

Koeireuteria, Sistewtere, Sirannemittbeeren.

Die reifen Siapfeln gefrurbt, birnformig; die Ståmmdsen unten blattlog, oben blatteris; bie Slátter evígnlid), loffelfớrmig, Durch. fd)einig, bollenforrmig gebculft ( 10 l)n. fan. Dige SBseidepläze, Koblmeiler, Steine in feuditen 230 lounger. Die Sapjeln finden (id) faft immerbin)

\section{L. \\ Laetuca, Eattid), Salat.}

A. Die Slatter su ber Mittelribe frachlidft, gefiesert jerichnitten

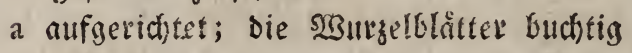
(Whin. an 3åmen, Felowegen. Z3!b:.

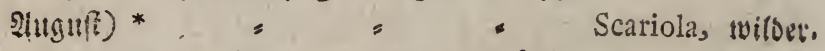

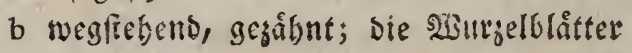
gants = " " virofa, ftinfender.

B. Die Slåtter volftemmen glatt, langetfors. hygrometrica, ge' meine. mig, gefiebert gerfinnitten; die Etucfe ge.

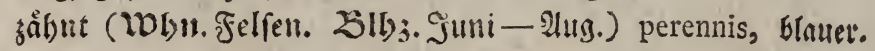

C. Die Slintter oben rundlicht, am Stengel bergformig; vie \$lithen in einem frachen Etranfe (LVIn. in allen Sirautgarten) * fativa, gemeinew:

\section{Lamium, ₹aubneffel.}

a Die Silfécennerve bes Scelmes gabelfơrmig;

Sie Slatter berjfơrnig

I etwas fumpfratecig; Der Etengel etwas friechend (Lobn. Aluinen, Etrafien điberall. Zlbj. bei) allen Itrten Frúb. ling bis serbft. Gebr. alle arten ben Sienen und Den (Shagfen) * . maculatum, ge* fleḉte. 
2 f(j)arf gugefpiazt; Der Ctengel aufred)t

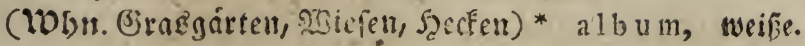

b bie Rilfennerve bes Seflmes einfad);

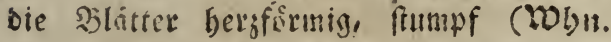
Gartenlano, alefter) *

$=$

กิurpureum, cotbe.

c bie Slätter unter ben Sllithenquirlen ftiels log, umfaffeno, frumpf; bie Slamen weip ober blafroth (Whn. wie ober $)^{*}=$ ampexicaule, um: fontende.

\section{Lapfana, Siainfobl.}

a Die Frudt ectig; Der Etengel åftig; bie Slötter enfơrmig gefrielt (Wobrt. Aecier. 2315:. Nay - Juli. Gebr. als jung frifit ifn Das Rillovieb) *

- communis, gemei:

b bie Edhifte gang einfach, Ibluthig; bie 2Isurjel Ifopfig; bie fdinitten (IDbn. Raubborlger)*

c bie Schafte aus ber 2 (3urjel, meifteng gab= foctida, ftinfen= ber.

licht, aufruairts allmáblig bickel, wollfom: men uacft (Whn. Feloer. Zlly. 2lıg.) minima, Eleinfter.

\section{Laferpitium, Saferfinat.}

a Die Slâtter breynal gefiedert; bie lezten Slätthen jufanmflefseno, vielfpaltig; bie Stưde foharf lanjetfórmig (WOln. feuchte Gebirglvicietr. 2315;. Juni, Juli)

b bie Sllitter gefiedert;

bavaricum, baicps (i)ie?.

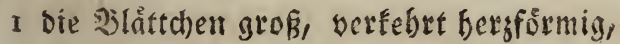
ftumpf rägezábnig; die Ságejáne an ber Epiţe fursftachlicht (Woln. 11.21 ) wie oben)

2 bie lezten Slättd)en geftielt, fpisig ev: latifolium, breits formig, volffommen ganj (Wolsu. wie oben)

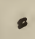

Sil er, fepelartis
ges.

Lathraea, 


\section{Lathraea, Schuppenwury.}

Der Stengel volltommen einfact); bie Blú: then uiberbangent; bie Unterlippe zipals tig (Whn. Wiejen, 2uen. 25lbj. গRay,

\section{Juni)*}

a Die Sllutthenftiele Iblůthig;

I bie Sd)lingen blattchenlos; bie Salin.

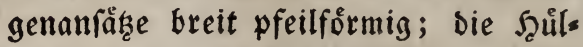
ren jugerpizt, 6faamig (WOLn. Şecfen. 2llb3. Juli, 2uguft) = " Aphaca, blattlofe.

2 bie Sdlingen 2 unb 4 blatterig; bie

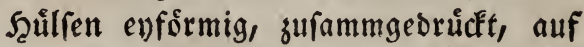

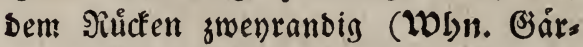
ten. Gebr. Epeife, Futter) " fativus, znfme.

b bie Bhęthenfiele 2blưthig; bie Salingen 2 blåtterig; bie Ṡlåttd)en enruno långlid)t;

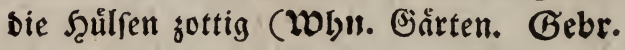
Sierbe, (Beruch)

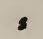

c bie Slưtbenftiele vielblưtbig; rqua m maria, globie.

a bie Sd)lingen 2 blátterig,

I bie Blattd)en länglid)t enformig

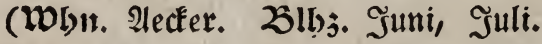
Gebr. Die ŞSurgeln zum Sd)weing. futter, fonft ein bofes linfraut)

2 bie Slattchen lanfetförmig, nervig;

odoratus, wobts riedjende. bie Sd)lingen gang einfact; bie ßlatts anfirke fponbonfơrmig (Wobn. STies fen. Slly3. Juni. Gebr. gutez Sutterfraut) * = = $=$ pratenfis, ges

3 bie Blâttchen langlangetfórmig, 3 ner. vig; (ber Stengel geflugelt) - fylveftris, $\mathfrak{T a}$ alo, platterbje. 
4 bic \$lattchen breitlanjetforntig, 5ner. vig; (ber Stengel gefribelt) latifolius, beeit=

$b$ bie Ed)lingen $2-4$ blåtterig; bie \$låts Glätterige. d)en finmal lanizetformig; Der Stengel gefiugelt (Gebr. Futter)

- heterophyllus, grasblitterige.

c Die Edlingen 4-66látterig; bie ß̧latt= anfäkze balbpfeilfơrmig; Der Etengel

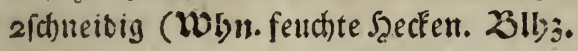
Iแแni)

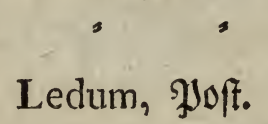

Ledum, $\mathfrak{d o f t . ~}$

Die eingige befannte Int wolnnt in Moré: ften, blitlst im Suni. (Gebr. jum Gers ben, miber Notten unb Echaben) = paluftre, Rien

Leerfia, Seerfie.

2 Die Rappe verfebrt gloctenfórmig, suges pistąt

a am Ranbe unten franjenartig gezånnt; Die Sdeibe ant Grunde bes Siaprelftie. les waljenformig " " " ciliata, gefranjte.

b glattransig; bie Echeibe am Girunbe

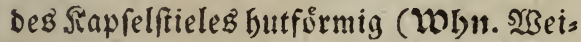
Den, graslofe झlåke; bie Srapfeln rei= fen im f̧erbfte) $=$ : " vulgaris, gemeine.

b bie Siapfeln aufred)t, enformig; bie Dectel fegelfórntig, f(c)uabeli̊tnulich, etwas gebs: gen; bie Slátter länglid)t eyformig, fd)arf zugefpiţt (Reife im Iipril)

cufpidata, ges

c bie Sapfeln gefturgt, ifre Stiele furg; bie ipiste. Slåtter fdimal lanjetformig, in ein langes fraar aublaufend (WDbn. Steine, alte Nonuern, Dächer. Reife. Jruibling)

pulvinata, polfter: fortmige.

Lemna, 


\section{Lemna, Entengrůn.}

Die Blåtter enfơrmig,

I larijetånnlich, geffielt, aนs ibrem Nittel Slatter treibeno

2 unten flad); eine cinfadie, cingelne Siurgel

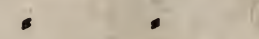

3 unten balbfugelfórmig; mebrere eingelne 2Surzeln

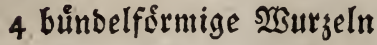

(Alle biefe 4 arten wobnen in Tei. d)en, So̊chen; blůbell im JuniEept. und geben ben Enten und ङ゙ånfen, und mit Sileven vermifht aud) ben Scứntern ein angenebmes Futter.)

\section{Leontodon, sowenjafn.}

1. Der Schaft âfig; bie \$laitter lanzetfor: mig,

I gesannt ; bie Slletthen bes Şauptaftes in Dolben; Der Stengel am Girunde fart fteifborftig (Woln. Wriefen, $\mathfrak{x}$ eiben in bergichten Giegenden. 23lbj. Spåts (ommer)* : , .

2 glatt, mit langen 3áfnen; bie \$llưthen: fitiele geid)uppt (Whyn. und 23lb). wie oben) *

II. Der Echaft Iblütbig,

umbellatum, bots Denformiget.

autumnale, hetbft: lidjet.

I blattlos,

a mebridjuppig, unter ber Sllütbe bicfer

( $\mathfrak{W}$ bu. Gebirggegenden. 25lbj. Juli) pyrenaicum, pys

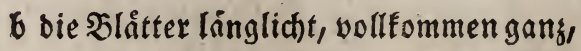
tenái的er. mit kursen bervoripringenden Báb. nen (Woln. wie oben, audh Boralpen.

23llj. Juli-Sept.)*

incanum, gratet. ( rơb. 
c róhrig, weich, faftig; bie Blåtter lanjetformig, grobjäbnig, glatt (ZWlin.

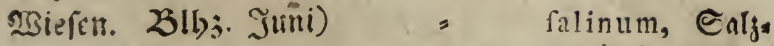

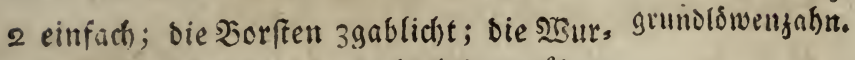
zelblätter niederliegento, bud)tig gejåłnt, borftig

3 Dic Blitter gefiedert serichnitten, glatt;

hifpidum, fteifs borftiget.

Der Sielch) glatt, ober mit feltenen Sors ften (Mhn. W3alsungen. Z3lbj. JuniSinguft)

Leonurus, Eowenidjwanj.

Die Stengelolatter faft banoformig zerfdnits ten; Die Etúcke lanjetformig, gezådnt; bie Slîtter an ben Blithenwirbeln lanjet:

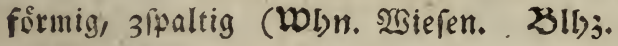
Sulli-Sept.)

\section{Lepidium, Sireffe.}

$=$ Cardiaca, gemeis net Şerzgepann.

I Die Sllitter enfơrmig, lanjeti̊nnlid), un. zerid)nitten, fågesåbuig, bie obern glatt= randig (WDhn. Birten, uno mo Giarten. mift biugeworfen wird. 25lbj. Juni. Gebs. wie bie folgende 2trt zut Epeife) *

2 bie Slitter línglicht, vielipaltig (MObnt. 2lbj. uno Gebr. twie oben)

3 bie \$ुluthen 2mánnig,

danubiale, Dos nauloroenzann.

a nit Slumenblåttern; bie Blâtter fans zetformig, fågezånunig (WWhn. Echutts baufen. 3lyj. 2lug.) = " Iberis, wille.

b blumenlos; bie sisurzelblåtter gefiebert; bie Aftelåtter linienformig, vollfommen gan. (Whan. Ruinen. Slbj. Eommer, Şerbif)

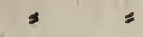

ruderale, frits Eenoe. 


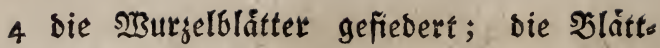
c)en vollfommen ganz; bie Blumen lån. ger alz bie Sieldhe; bie Echåfte naft (WDln. Alpen, an ufern. 23llj;. IprilSept.)

\section{Lepra, Gtaubntoos.}

alpinum, 2rlpen. ferefle.

I Edwary,

a ftaubig (Woln. alte biebnilbe, Edhiefers fteine)

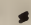

antiquitatis, (d)warzes.

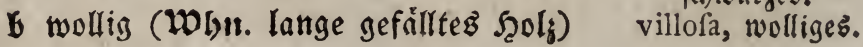

2 gruinlichtgralt, fo lange $\mathfrak{e B}^{\mathrm{B}}$ feucht ift, tro= d'en weiß (Wobn. Saaumrinben) = incana, beftiubs

3 f̉nefelgelb, abfårbend (WWl)n. Saumrin= teร. Den, Felien, Flecten, bie Unterfeite eini: ger Pfianjenblatter) " " candelaris, iffwes

4 graggrůn, in fleine f̧ณ̊แfden gefammelt felgelbes.

(Wolni. ain Fuf Der Giebrude, an ber (Erbe, in Slumentópfen) $\quad . \quad$. botryoides, tralls

5 fpangruin (Whly. tief an ber Eroe abge: bauene Stocle in feucten sisaloungen) aeruginofa, grun:

6 freibenweiß̄; unter bent Suchglafe ftaus ipanfầrbiges. bige Rörner (WDln. Saumrinden)

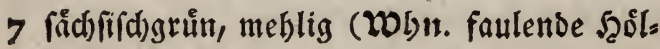
zer)

8 mennigroth (Whit. feuchte Nautern)

lacten, falfartis ges.

phofphorea, leuchtendes.

miniata, rothes.

9 blutroth, gelb abfärbend, nach Beilen ries c)eno, im Allter eingeichrumpft, graut (Woln. Steine in $23 a l$ bungen) s Iolithus, Biolens

Io rofenfårbige, faubige, gebåufte Rlump. moob. d)en (Woln. swifhen ben flechten an (Fi= d)enftámmen)

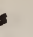

rơfea, rofenfåtuis gอริ.

Leskia, 
Leskia, Serfie.

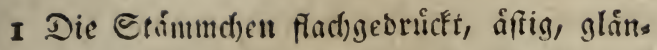
jent ; Die Bläter benderfetts 2reibig, an

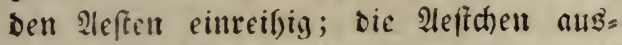
warts idjmåler (WDbn. Felien, Daumurin= Den)

= complanata, flad)s

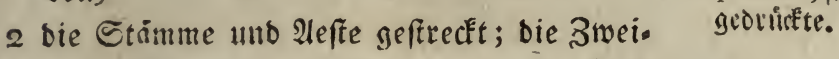
ge auffebend; die Epitzen im Irodfnen uberbangend eingebogen; bie Rapjelfitele einzeln; die Slätter ppişig lanjetfơrmig (Woln. Binune)

fquarrofula, ftraus

3 bie Ståmme und Iefte fried)ent, gebrángt; bige.

bie sweige aufrecht, âftig, gebróngt; bie Hefte fadenformig, rumblid)t; bie Slitter ramal langetformig, fehr fisigig (wobn. Saumritrden, ath an ber (Frbe) = myofuroides,

4 Die Etñumd)en bielåtig; bie Aefte ant mâuej id) wainzige.

Ende nabelforrmig, verfdumădtiget ober bicklid)t; bie Slátter eyfờrmig, Ioffelelåt)

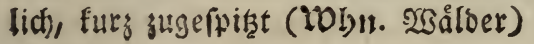

radicans, vet: lit)máchtigte.

5 bie Crámme und Afte friedsent, wernor: ren; bie Sweige aufied)t, fadenformig; Die Sappelftiele aus bem Etamme, etmas ưber zmeigelang; bie Slatter baarfein fpis kig; die Sielctblátter glüfer mit einem Enoshare (LWln. Saumwurzeln, Steine an feudten Stellen)

6 bie Ståmmdien friechend; bie Aefte aufs velutina, verwor: tene. red)t, ziemlid) einfach, bie 3weige fpar= fam; bie Slatter Ianjetformig, zugefpiţt;

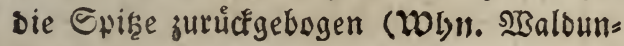
gen)

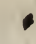

flagellaris, peit: jidenåbnlid)e. 


\section{Leucojum, fruhtingsglodé.}

Die Edyeite I-2bluthig; Der Siriffel Eolbents formig (bobn. fette șiefen uno 2luen. Zllyj. Febr. - Aipril. Grbr. Den Sienen febr willtommen)*

vernum, wahte.

\section{Lichen, Sledte.}

1. Sdjorfe mit şarzen.

A. Eine unablósliche

a weiße Srufte

a mit gleichfå̀tGigen, unregelmåzigen in Alter mebligen $2 \mathfrak{s a r j e n ~ ( W W u t . ~}$ an ben Rinben ber Suchen)

fagineus, ๖utren:

6 mit verichiebentlid) fich ourdfrelts fledfte. senben, fatwirjlid)ten ober (d)mat. zen Strichelchen (WDLn. Saums rinben)

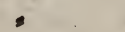

t mit rotben, geftielten Singeldhen (Whin. Fonnige Shonftellen)

b unrein weife, faun merflid)e Sirufte; fcriptus, હ(j)ift: fledtite.

b a c o m y ces, Fleifâfarbe. balbfugelformige, burch und burd) líchwarge 23 argen (Wobr. Bauntrin ben) .

,

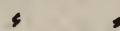

fanguinarius,

e grůnlid)tgraue sirufte, mit ungleid) Vlutflectite. großen, gebrúdt balskfugelformigen fhtwargen SBarzen (WDIn. Öranit uno 2 3acken)
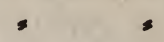

petraeus, 2 isacken:

d weiß̧licht âfdengraue Sirufte, mit eis fletite. ner gleidfforrmigen (im feudbten 3us ftanbe blapggrüen) mebligen Subs

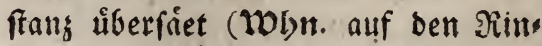
Den bes Roṕfaftanienbaumḃ)

carpineus, mor: telformige. 


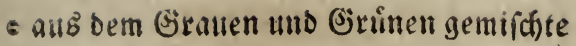
Sirufte, mit burchaus fdiwargen balb.

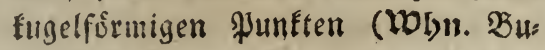
chenrinoen)

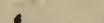

f grunlid)t afd)enfärbige, rinbenfơrmige limitatus, bes grånzte.

Sirufte, mit zertheilten braunen Sias pitelfen auf Stielen (WObn. Ed bie. fer)
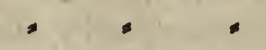

g grůnliḑt meiß̧e, neflige Srufte, mif fungiformis, pilz. ingnliche.

friellofen, foulfelformigen, im Alter gewolbten, weiskandigen, gelben Sas

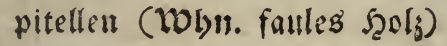

h graugruine,

lentigerus, \&infen; fleciste.

I meblige Rerufte, mit rotben, fitel. lojen, in Alter gerwellten Siapitels len (Wh). Sinden, faules frols in szaloungen)

2 mit braunen geffielten Rugigelden

(Whyn. Iettige Stellen) = fung iflorus,

i roothlichte, falfartige Sirufte, mit gleich). elveloides, falteni pilzformige. brame. fărbigen, fugelförmigen, oben Dur(h):

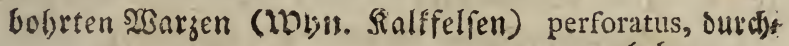

k fact)e Srufte, mit fdmarjem, allmäbs bolitt. lig fich) vergrsfisernben, endich in eimander fliešenden \$unften ( $\mathfrak{W}$ bu. Salffelien)

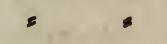

1 gelbe Serufte von unbeutlid)er Geftalt, confluens, vel: fliérenoe. mit ftiellofen, fatt oraniengelben Ra. pitellen (Whn. Eteine, Manern) fulvus, gotogelbe.

mn glanzlofe, meblige, tiefichmarze Srufte, mir gleid)fárbigen, balbfugelförmigen

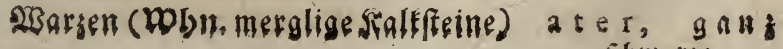
ifiwarge.

n faubo 
I faubâfnlidbe, ounfel nichengraue Srut. fie, mit balberbabenen, rantojen, fattictwargen $\mathfrak{W}$ arjen (Woln. Baum: rimben, Felfen) " =" fufcoater, fleins

o meblige, fhtwarge serufte, mit graus punźtige.

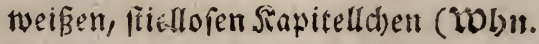
merglige Salffeine) = " atroalbus, weiøs

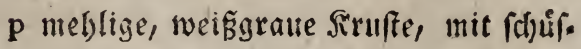
blüthige. relformigen, in Alter gewolbten, gleidjeandigen, lattichwargen Siapitells d)en (Lohn. Siof̂́aftanienbaum)

cinereofufcus,

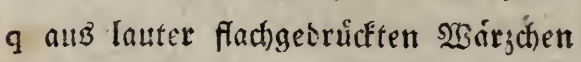
befiehende weisgraue Sirufte; bie aufs flechte. blübenden fiapitelld chen nur in Mits telpunte auffiţend, mit weif̧em, enos lid) geferbten Siande (WOLnt. merg= lige Salfiftime)

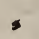

B. rußige, aus \$unften uno furgen, mit (d) warzem Stanbe gezeidneten finien beftebende Flecfe (Woln. Suthenrinben)

II. Sdyorfe mit Sduiffeld)en.

A. Eine unablóslid)e

I weislichtblaue Riufte, mit gleidhfér. migen runben Sỏchern (Whbn. RalEs felien)

2 weiglid)te, meblige Sirufte

a mit olivenbraumen, weislichtgeran, Deten Sdhuiffelden; ber ?anb fein geferbt (WDlt. Sauturinden) fubfufcus, oliven,

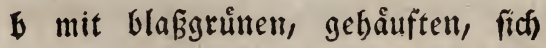
erofus, ausgeftef: iene.

cinereus, afdiens เociß̧e.

rugofus, iffrwarg: tunflict)te.

Sidwarglinjen 


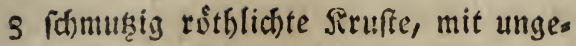
fitelten, fteinbatten, oranientotben Sd) uffeldien (Wobn. Minmor: gebirige)

- rubellus, tosths

4 gologelbe, meblige Trufte; bie Edhuf: licite. feldben roth, mit blófferm, bicklidten Sante (Lobn. Sialefelien) s

\section{5 gralle,}

ventofus, Sialf. felienfledite.

a barte, mehlige Rrufte; bie Şar. jen punftfơrmig, fotroarz, burct)= bobrt, werientt, endlid) hervortret. teno (Woln. wie oben) = punct iformis,

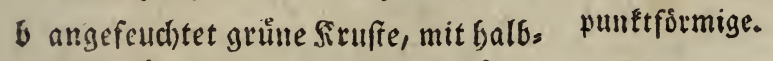
fugelformigen, blaß̧en @chůffel. chen (Wobur. wie oben) = urceolaris, fluge

c feine Srufte; bie Edxiffieldsen grau formige.

fleifhfâtbig, mit weiflid)tem গian= De, im Alter Rapitelicben ( $\mathfrak{W b}$ b. Baumrinben) - pallidus, vets

6 meblige, vergängliche Srufte; fitel. blabte. lofe, in Der Jugend vertiefte, fleifhs: fårbige; im Alter etwas "sewólbte,

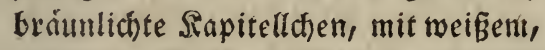
geferbten Rande (Wolnn. an ber Er: De in $\mathfrak{S a ́ l}(b e r n)$

-

pezizoides, bedjets

7 gelbe, meblige Sirufte, mit allmáglig pilzóngntiche. (ií) båufendelt, gefáttigten Echtiffels

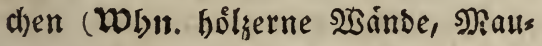
ern von Ennbráufern. Gebr. zum Belbfärben) $=$

candelarius, gelbe

8 gelblid)t grůne Srufte, mit rothen, Sairbefledite.

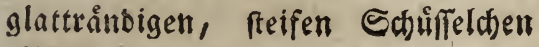
(DDLir. STalffelfer)

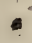

cupularis, flad): tellevfledite.

B. eine 


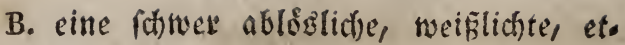
mas biflidate Srufte; bie Sditiflelden fitellog, fomairzlidst, mit einem graten,

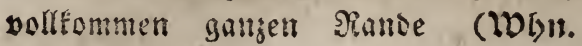
Strüucher) - . . nigricans, fiduates

C. obne Sirufte, mut afthengrane, fitellofe, (id)te. linfenfơrmige, in vcrtieften Mittel raume formarge Cabuffeld)en (WObn. Steitit)

lentiformis, grauer fintents ifjolf.

III. Eduppige flechten, mat હdfuiffelden.

I Eine unablogsliche, afdhenforbige, blât: terige Sirutee, mit georångtan, báufigen, fdrmairglichten, Greitlicht gerandeten, ges ferbten Echuffelchen (WOWr. am wilden Raftanienbaum)

nigrefcens, ointert farbige.

2 eine unten und oben fitharge, (frifd), oben ratt gelogruime) faft blâtterige Srufte; bie Enden ber Silntter furr gablicht, linienfơrmig; bie Edruifield)en gleidffirtoig (IDGn. bodhliegende natte Felfen)

3 eine (ch) ablósliche, blítterige, grůn. fahlunenfis, $8 \times 5$ : lunerfled)te. gelbe Sirufte; Die Blattfticfe vielfpals tig, lappig; Die fitelloren Rapitellthen ofrefsrmig, bottergelb, endich) feriffer. formig gerandet (WOLn. Selffeime) ochroleucus,

4 eime grune, mebr ober meniger ablós, gelobunte. lid)e ichuppigblätterige, unorbentlich úbereinanber liegende Rrufte; bie Sdhuifs feldsen gleidffarebig, in gelb verblafiend (Whit. Diure Birnbaumifite, abgefals lene Ziweige)

muralis, Naueri fiectite. 
5 fruftenlofe, fleifdrotbe, in ein, meiftens

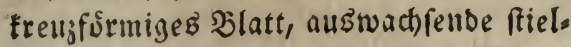
lofe Gduffeldhen mii meifiem Ranbe (Whn. an bet (ErDe) * $\quad$ cruciformis, fieufformige.

IV. Blätterige, aus einem Snittelpuntte im Sreife auslaufende Fled)ten mit $€$ (h) feldsen.

I Die fduppigen Blatter in einem Sreife, graw, langlicht, tief zertbeilt und laps pis, lunten fanrórgliat; bie ftieflofen

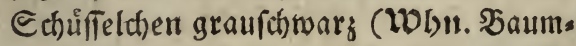

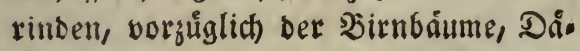
c)(r)

2 faft unablósliche

Atellaris, Stetn flecifte.

a blåtterige Echuppen, oben grau, ober (angefeuchtet) ichmutisig grinlicht, uns tell fhwars; die Edjuffeldhen in Nit. telraume gebauft, forwärylicht grau, grunlicht geranoet (Woln. \$̧aumrin. Den)

b chuppenfơrnig ưbereinander liegende, olivaceus, oliven: fárbige.

faft gefiedert zerfá)nittene, muß̧oraune, (feud)t, apfelgruำe) Vlăttchen, mit braunen, (feuct)t, fdimarzsrotben) ges randeten Echuffielchen (WDLn. SH: c)en, Dbftbåume)

- omphalodes,

3 bie ßlatter nach alfen Richtungen ber. grabgrůne.

umliegeno, glatt, fchmal, angedruidt, mit \$unften Durchbofrt, lappig, an ben Enben etwas aufftebeno, und meblig aufblübend, oben reisgrau, unten fhwars (Whn. Saumrinben) - pertufus, outdo 4 Die Slåtter úbereinander liegend, isfjelte. 2 vielfach lappig, 
a unorbentlich, mit neţformigen, ets babenen, mebligen Sinien, unter (c)roary, mit gleid)fä̀bigen, zabl. reiden Sorften (DOLn. Saum. rinben, Eteine. Gebr. jur \$urs purfarbe)

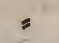

b fraus, angenebm bleidjgru⿰!, unten fdhuars; die Sdhiffeldsen grofi, unpforming (WDLn., wie ober. Gebr. Farbe)

c (t)uppig, aus einent Sittelpunft caperatus, rofers formige.

laciniatus, ges fricifte.

fommens, mit gleid)fârbigen, in Der Sitte gebrăngteru Echuiffel.

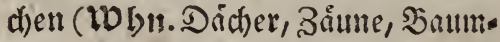
rinben. Bebr. Die Biegett frefs fen fie. Farbe) $=\quad$ parietinus, $\mathscr{\text { TBnns; }}$

ouffebent, breitlappig, fraub, oben flectite. glatt, unten ureben und (c)ivars (TDLı. Saumrinben)

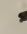

b gablict), linienfortmig,

a fluppig liegend, etwas aufitebend; sie Stucke faidig gefiangt, oben int feudben వuftande gruin, im trodinen grau, unten fómuşig

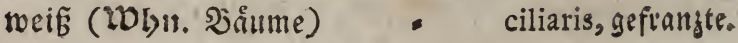

6 iu einen Sireife liegend, an ben Spisen aufgeblafen, oben afchens färbig, untell fowwarz Sămne, Steine)

(WDLtr.

5 blattrifulich, faft linienformig, serfanits glaucus, meets gixine. ten, aufred)t, mit langelt Sorften ges fianjet, trodfen, uno feuct) weiblidt (DD)11. Sdblebenftămme) 
6 bas silatt aufliegent, friechent, vieltheis Iig, breit, sben mildtrabmfơtbin, unter formar; die Echúfieldhen groß̧, braun. roth, fwarfam (WWhn. Felfentrimmer) perlatus, sepers

7 ein auffethendeg, zerichliffents, mit $\mathfrak{B e r s}$ lete. tiefungen ausgebsolteg, allentbalben

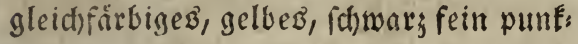
tirtes Slatt (Won. Faumrinden. Gebi. Fatt Der Drfeille, Fânerinoog) nivalis, Sdjnees

8 ein unordentlich jerriffenes, aufliegens flectite.

Des, feuctit, uno trocken weißes Br!att; bie Etudcte länglidft fich aufridtent, geframit; Die Schulfeldhen friellog, fchnars (Wobn. Edjlebenfauden)

hifpidus, tano, borfige.

V. Straud)âbnliche Slåtterflectren.

I Die Blatter żemlich auf:ed)t, grůnlichts gelb, auf ber Unterfeite uno an ben fef) fraufen Råndern fattgelb (WDbrr.

Sisachbolderftaltoen. (Oebr. fâlbt gelb)

2 bie Blätter auffebend, linienformig $\mathfrak{g} a=$ belábnlid) zertbeilt, beyoerfeits graus juniperinus, T3 a th boloels fledite.

weifi, an ben Ceiten mit mebligen fla. d)en Sisarzen befejt (Wobn. Sónme,

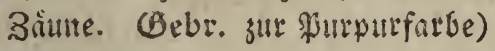

3 bie Slátter aufrecht, vielfaut) âfrig zers farinaceus, meø. rige.

fchnitten; bie 2efte linienformig zers fornitten, ůberall gleidhfórbig, mit lång= lid)ten Giribchen; Die lejten Ilefteden angefpigt (WDbu. b̊rume)

4 bie Blätter breitlinienfơrmig, gablicht, calicaris, geffinase belte. runglidt, einförbig; bie Schuiffeld)en an Den Seiter und Enden, endict) in grofere, Dem Blatte gleichfärbige, faft gefticlte Seller ausmachfend (robn. (Eichen)

fraxineus, (Eichen: flectite. 
5 bie slâtter aufitebend,

a gablicht âftig; bie Ilefte linienfơrmig, an Den Eeiten zuruicfgeroflt, oben grall, gan mit mehligen Siornern bebedit, unten tief fetwarz; bie En. Den fpisig (WObn. Daumrinden) furfuraceus,

b lappig zerfanitten; bie Etricte ges Elevenartige. framjt; ber Fü ber Plange rotb (Whin. an ber Erbe. Gebr. Epcife

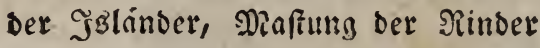
und Schweine, jur gelben Farbe) islandicus, islán.

6 basే Blatt aufliegend, vielfad) lappig Dilifie. gefpalten, oben mit Giruben neģfơrmig befäpt, unten zottig ( chen, attc) Slabelfols. Gebr. miber

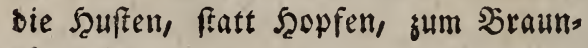
fơrbeli)

-

VI. Biallertig hruttige flect)ten.

I Die Slåtter gebảuft, Ernub, auffétend

a fumplappig, obne $S d$ úfield)en (Whn. alte Nauern, Baumrinden)

b fdrwarggruin, mit gleichfärbigen, ge. băuften Schúffeldben (Wobrt. Mafte Felfen)

2 ein vielfach) zertheiltez, (d)mallappigeb, (d)warggruines Blatt, mit (ch)wargrotben Ed)uffeld)en (Hobn. Selfen)

VII. Berufte, blåtterige flectiten, am æittel. punfte auffitizeno

I ein lappiges, ftumpfrandiges, gefranss

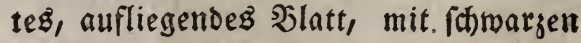
Sügelden und \$unften auf Der Dber. peite (WOLn. Felfen) pulmonarius, $\mathfrak{R}$ uns. genfledite. granofus, tirs nige.

crifpus, ftaufe. 
2 ein rumbes, ftumpflappiges, oben ruß̧,

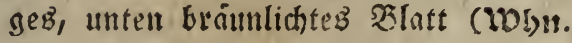
Steine) = . " = deuftus, mäige.

3 ein gallertig bäutiges, breitlappiges, Dưnes, in ber Jugeno etwas burd)= fdheiniges, unten idiswarges, oben trocken graues, feudse fattgrúnes, in alter fdwarzes Slatt (Mbir. Båme)

4. Die Slatter bachsiegelformig ůbereinan. Lactuca, falat: blátterige. ber liegent, ramal, gefrnurelt, an bey. ben Eeiten glatt, am Sande geferbt (IDGur. natte Felfen)

VIII. Seberartige flechten.

= polyphyllus, vief Glatterige.

I ftuch,

a unten aberig, einfad) gruin; bie Sdillde am Sianbe sageredt, idswairs. licht (TObn. an ber Eroe in feuchten (Biebuirchen)

-

venofus, aberige.

b die Schilde foruffiflformig, braun, in Das Slatt eingefenft (IVb)i. zwifhen bem Mioofe auf Felfen) * " faccatus, factfor:

c ututen abernlog, in ber Stugenb graus mige. grůn, im Ilter rotbbraun; bie Sdbils be eingeln, tellerfismig, fdymal geralts bet, unten fammetartig, im Alter nach) ber Quere linglicht (WWlyn. an

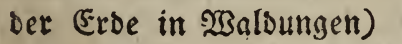

2 bie Schilde an dent Enben ber Rappen horizontalis, was getedte. aufifebeno,

a lappig; bie llnterfeite aberig; ver. fhieben fårbig (WDbu. unter bem IRoofe)

- $\quad$ caninus, Şunbe Fectite. 
b fparfame STarzen auf ber Sberfliche; in feuchten 3uftanbe grasigrin, tros cfen stau (WDltr. in Şaldungen an Der (Erbe)

,

IX. Rorallenflechtert.

I Fin gan' cinfaches, fpitsig sulaufenbes Scorn obne Sduppen uno Slätter fimpliciffimus,

2 ein parfam åftiges, einfaches, bobles, nattes, mebliges f̧orn

3. cine einfad)e, oder faum affige Reule

4 eine einfact)e, oder wenig affige, nafte, bald gefornte, bald in einen Sedber auslaufende Flechte

5 eit Eurjer Jecher,

a einfad, an ber Miuntung geferbt, unb mit braunen Rigeld)en befegt pyxidatus, כes

$b$ an ber sionoung geftralt, uno mit rotben sugeldien befezt

6 ein langftieliger Secher,

a mit rotben Singeld)en an ber Maits bung,

a einfach, glattrandig, ant Sanbe proliferirent ,

$\mathfrak{b}$ åftig; bie Aufenfeite mit Schup= pen befezt

b ziemlich einfach), mit einer jiemlich ganjell, Dunfelförbigett, engen Min. oung, am Sianbe proliferirend

c mit gejąbuter Nưnoung, einfach, am Siande oft proliferirend

d faluppig, sftig; bie Ninoung mit braunen Sigeld ben befegt

(Wbu. alfe diefe Arten an mobernben Stocken uno פaumtourzeln) a phtorus, Sch wa mm chen: flectite. einfadffere.

polyceras, mebrs boinige.

clavaeformis, feus lenformige.

deformis, geftalt: loje.

\section{cherfledite.}

cornucopioides, fullbounábnlidfe,

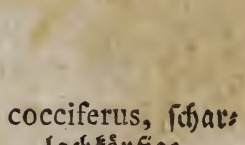
bointe.

fimbriatus, ges zífnte.

impetiginofus, aนรี|สุรุige.

X. Strauct)= 
X. Straudbignlidje Gled)ten.

a Did)t,

I vielgablidst, weiff, am Grumbe rofts gelb (Wobn. Die ?iinne, nuB welcher

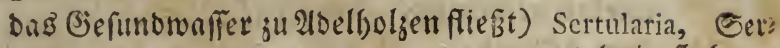

2 febt åfig, mit fiugeldhen an ben Ept=

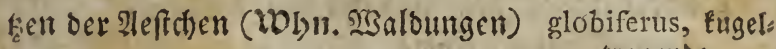

b róbrig, tragende.

a reble effig,

I an Den Id felln burchbofrt; bie lezten 2leftchen febr fury, ipirgig (LOLn. an Der Erbe in Rabels bof(zern) =" " uncialis, Futrys

- bie 2lefte überbangent; bie lestent aiptige.

2lefthen an Der Epizze braumrotb, abgeftumpft (WOln. wie oben) rangiferinus,

3 blåtterig, (aud) naft); bie lezten Fiennthiermoos. Ileftchen ppişig mit siugeldhen (Whln. trockne Eannenwaloungen) Co rnu cervi,

b fparfamer gablid)t, åftig, ziemlid) naft; Şirfithgornfled)te. Die lezten 2lefte pfriemenförnig, mit Sirigeldhen an ber Epitze ( obeir)

XI. Sanแmbårte.

fubulatus, pfrie. menformige.

I 2ufrect)t, siftig, bidst,

a an Den Enden mit gefrangten Tellern floridus, fterntras

b gelb, rotbmerdeno; bie Aeffe pfrie= menformig

c zerftreute, meblige sisarzen

2 berabbangeno

a mit baarfirmigen $\mathscr{A}$ eftchen; bie $\mathscr{A d})$ s reln zufammengebruicht; zerfteut mef) lige Punte

$=$ jubatus, mábnen: formige. 
b rehr åfig, febr lang, mit gerftreuten

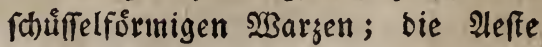
runblicht

c fabenformig; bie Aefthen bin unb barbatus, rangi mieber gefrümmt, ein weitlo̊cheriges शeţ bilbent

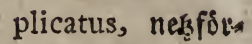
mige.

3 unorbentlich bin unb mieber gebogen, fabenformig, etwas affig, bicht, mit långliditen Bruibchen an ber Dberflidhe chalybeiformis, (Whit. alle auf Baumftammen) Drathformige.

\section{Ligufticum, siebftocfel.}

I Die slitter 3theilig, bie Theile zwenmal 5theilig; Die Enoftucke 3fpaltig, fågezât). nig; bie llmichlagblåtter am Ende ganj, und gefpalten (Whltw. Waloungen. Zll)j. Șuni-2uguft)

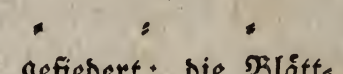

2 bie Slätter Doppelt gefiebert; bie Blatts d)en feulformig eingefdnitten, bie Stuidfe gang (TOhn. wie oben. 23lbj. 2tuguft)

\section{Liguftrum, Şartriegel.}

Die Blătter enfơrmig, lanzetifnlid); bie

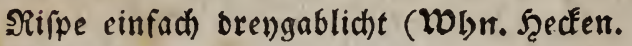
215;. Juni. Gebr. zu Sceffen, ben כie.

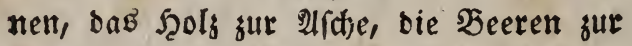
Surpurfarbe, Die 3meige ben Siorbma. d)ern) * :

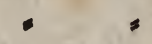

:

\section{Lilium, silie.}

2 Die Slätter im Suirl; bie \$lưthen ab. wårts bangend; bie Shlımenblatter zแแ rúdfgerollt (WDltr. feuchte 2 Saloungen, (chattige Şน゚gel. 23!hj. Juli. Gebi. Den Bienen) * brancionis, grof โยร.

\section{auftriacum, offe} reidjilifers. vulgare, sibein:
weibe. 
b bie Blâtêr getftreut; bie blumelt gladfent. firmig,
a ưberbangend, inwendig glatt
- candidum, weį́e.
$b$ aufrect)t, intoentig raub
(30b!. Gevbe in (Jårten)
bulbiferum, geuevs lilie.

\section{Limofella, Cumpffraut.}

Die Blätter länglid)t enfortnig (IDLn. ûber.

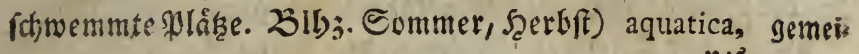
nes.

\section{Linum, \&ein.}

A. Die 2litter f(hmal langetformig wechjel. peitig;

a bie Relche unb Sapfeln fharf jugeipigt;

Die פZluntenblatter geferbt; Der Sten. gel sientlidh einfad); die 23 urgel eins jubrig (Wobn. Aleffer. 3lbs. Miny, Suni. Eebr. Der Safî ju Flach8, Der Saamen z̆ Del; sie Delfudjen zur Maffung Des Rinbuiebes) *

ufitatifimum, ges meinet:

b Die Reldbe geruntet,

I etwas bautig geranbet, fumpfipisigig; sie Slumenblátter vollfonmen ganz; Die Blátter glattrandig (WOLu. trodf́ne 2Biefen. Blhj. Miars-Nug. Gebr. laingern, aber grơbern flachs, fonft wie ber gemeine)

: perrenne, auss

2 bie Sieldje ftumpf, mit einer fefr fur= Daluetnoer. sen Epine, am Siande volfommen san; ; bie Slätter abroarts fteifbor. ftig; Die Dlumenblåtter reicht uns weitliuftig geferbt (Blb). Altguft, Eept.)

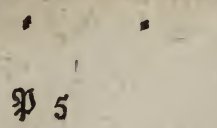

auftriacum, sfters reictifachet.

B. Die 
B. Die Slảttek faft borftenfơrmig, zerfteut, abmarts feifborftig; die sield)blitter fpis figig lanjetformig, unten mit Drưfen ge. franjt; bie Slumenblåtter långlicht (Błl);. (Eept.)

tenuifolium,

C. Die Släter lamjetfơrmig, mersig, mit Drü idjmalblatteriger. fen sefrant, bebaart (WObur. Stiefen. 3153. Juni, Juli) = $=$ vifcofum, Elebris

D. Die Slâtter evformig langetåbulid), gegens get.

ůber; Der Stengel gablicht; bie Slumen

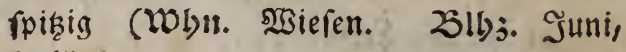
Juli)* Lithofpermum, Steinfaame.

a Die Saamen glatt;

I bie Şlumen Eaum linger, alB bie Seldhe,

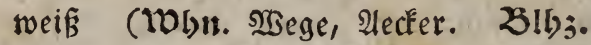
Nay, Juni. Gebr. Die Ņßurgel fårbt rotb) * , = ,

2 bie Silumen einigemale lónger als bie Sieldie, blau

b bie Saamen runglidft; bie Blumen faum lánger als bie Sielche; bie Slatter famal

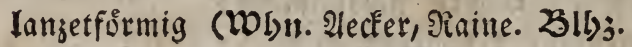
May) * :

Lolium, \{old).

officinale, Detten: birie.

purpureocaeruleum, purputs blauet. Purgitlein.

I Die Hehrchen einander beribtend, vielblu: thig, Die Slatter breit (WWhn. Aecker. 2lh);. Jinni)

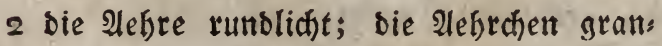
perenne, ausbau= ernoer. nenlog, genảbert; Die Slátter linienfơrmig

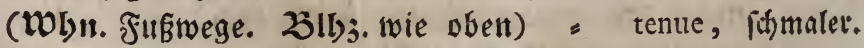
3 bie 2lebrdhen entfernt; bie Sranten fefro furs

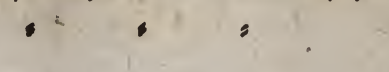

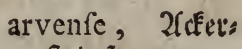
fteiniane.

remotum, entferns ter.

Lonicera, 


\section{Lonicera, Epefflitie.}

A. Die Plithen an ben Enden,

2 in sisteln, fiellog; bie oberften \$llatter sufammgewad) fen (WDhn. S̈irten. Blb). Juni, Juli)* , , Caprifolium, tvåts

b in eทrunden Iop fd)en, badjiegelfornig ithe. úbereinanber liegent; bie Silatter utm: vernact)en, einander gegenuiber (WObn. uno 23153. Wie oben $)^{*}=$, Periclymenum,

B. Swo \$lithen auf einem Etiele gemeine.

a bie Seerent verwachfen, roth, mit, ben Heberreften ber Doppelten Slluthe; Die Slätter enfórmig, zungeipizt, gan (Wobn. Gebirge, Şârtea. Slb3. Mial), Juni) alpigena, 2(tpen: b bie Secten nidft vermadfen, getrennt -ipef́lilie.

a bie Silitter efliptiad), zugeipizt, felbe unorbentlich fógezâfing, ober ant Siande gant; bie Scülle furber, als bie

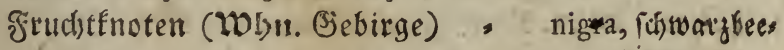

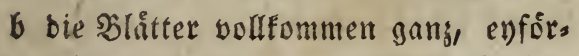
rige. mig, fammetartig (LDL)t. Scecken.

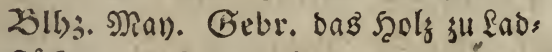
fiscfen, zur Feurung) *

\section{Lotus, Gdottenflee.}

$\mathrm{X}$ y 1 oft e u m, รุundรีtiridge.

a Die Shuthenftiele einğeln; bie Scúlfen 4fei. tig, gefligelt; bie Stengel am Brunde nies Derliegeno; Reld uno blátter bebaart (105n. Wiejen. 25!bj. Juni)

filiquofus, bâffi: b bie şlutthen in Şåuptdyen get.

I bie Şiuptchen vielblúthig; bie Sieldhe zottig; Die Blåtter 4 - 5 fingerig, fark bes baart; Der Stengel nufrecht (MObn.

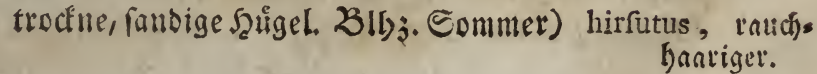


2 bie f̧ruptchen niebergebrickt; bie Sten; gel auffebeno; die Suilfen walsenförmig, regftehento (WDiti. Wriéfen, Sisetben.

23lbj. Sommer. Gebr. Den Bienen) corniculatus, geo

Lunaria, Mronobeil.

bornter.

Die Echottchen länglidht elliptird), etwas fitigig

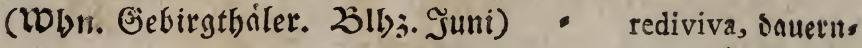

Lychnis, \&ichtroீ:ð)en. Der.

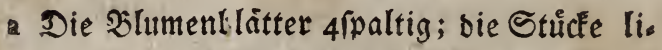
nienförmig (Whln. feuchte $23 i e\{e n .23 ! l y 3$. Juni) *

Flos cuculi, હ゙ঁ.

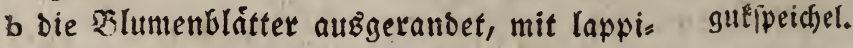

gen Эiågeln; Der Stengel oben flebrig; Die Slätter lang gezogen, langetähnlich) (WDlyn.

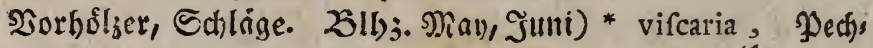

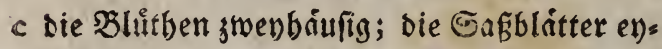
nelfe. fơrmig, lanzetåbnlid) (WObn. WBiefen, \&aub. bo̊lzer. Blb;. Nay), Juni. 2tum. alle brey Arten finb auf 2 Siefen febr bófe Unfråuter) dioica, gemeine.

Lycogala, MYGeberzotte.

2 Geftielt, gebriuft, gelbweiß (Wobn. an Siels lerbalfen, an Stollenzimmerungen in Serg. werfen. (Erfid). Das gamje Jabr)

b ein purpurrotber, faft flufigiger Tropfen gl ob ofum, ges meine.

(Whir. auf Steinen, auf ber Erbe. KRrfh. Srubling, Jerbft vor Af fgang Der Somme) purpureum, pits Lycoperdon, Staubpili. purtotbe.

1. Lleber ber Erbe

a birnfớrmig, weiß̈;

a bie Sberflád)e glatt, zur Saamenreife wagerecht, jerfuringetto (WObn. in Sæalbungen ber Oorgebirge) - fubalpinum, 230\% Eergsftaubpil 
b bie Sberflide fornig, ober feillwar. zig; zur Saamenreife an ber Spiţe fich) sfuend (Wobn. allentlyalben.

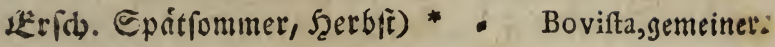

$b$ faft fugelformig ftruntlog,

a rstglidft; Die Dberflacthe glatt; bas Fleird) rotb (Wobn. faules Scols.

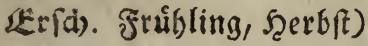

Epi d endrum,

$b$ einbaittg, warjig; der Snamenfaub

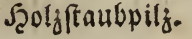

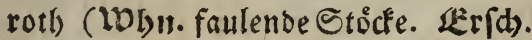
Şevbft)

c glatt; Die Niundung fpisig, gezåfint; pififorme, etofens formiget. bie Eybaut fernforrntig, fich abloffend (WObn. an Der (Frbe, febr gemein) * ftellatum, frern

- bie Evbaut vieltheilig; bie frutht cine formiget. aus Dem Saamen geballte Sügel (Wobu. im Mroder alter băume, nur einen

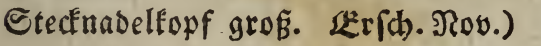

d Der CtrumÉ långer, als ber Şut, wals Carpobalus, $\mathfrak{k}$. geltwerfender. zenforrmig; Der f̧ut fugelfơrmig; die NRinoung ringfórmig, gang. (WObur. an Der (Erbe, in Şedfen)

pedunculatum,ges frielter.

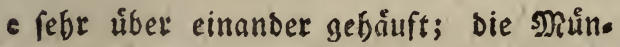
Dungen jerriffen, mebrtheilig; Der Etaub roftblichtbraun; Der gange \$il polfts rig, aus unendlich vielen, febr fleinen båutigen Zellen zurammgeregt (Whln.

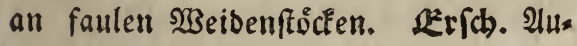
guft)

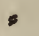

f gelb, gebåuft, brenartig, glatt; bie MRü" Dungen burchbohrt (W0bit. faulende

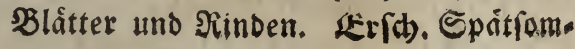
mer, গ̧erbft)

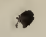

favaceum, waben formiget.

luteum, gelbet, g. fóma. 
g fomarogent, walzenformig,

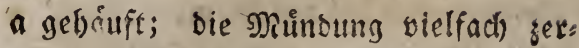
rifien, Der Etaub braun (MDly. auf Den Heften Des $23 e i$ boorng, wo er eis nen fleimen $($ d)wulfie mad). Eirib. Suli)

6 verrent; bie Minoung 4theilig: Die gregarium, gejels. liget.

Theile zerriffen, zertbeilt; ber Etnub gelbbraum (Whin. SBeiboornifie)

h unorbentliche rotbe, auf beuben Geiten Der blatter fidt)tbare, endich cinen bralls Oxyacanthae, 2Batíndornfaubs pily. nen Etaub nuzjduttende Flecte. (Why. Sirnblätter. SErfd. Spátrommer, feerbfit) "

i gebornfte gelte \$unfte mit vielfach) fers cancellatum, ges gitterter.

riffener Mindung, und oranienrotbem Enamen (Bohn. alf Der Siudfeite Der Slîtter des Şuflattichs, und ber Erle.

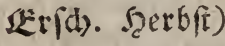

epiphyllum, şuf:

k gebauft, gologelb; die eingelnen \$ilse lattidiffaubpily. nach Der Sefnumg waljenformig; Der Staub gelb (IDLit. nuf der Unterfeite

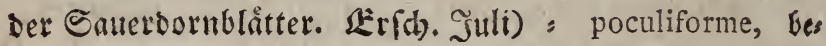

1 oraniengelbe, zerftrente \$uncte; Der d)erformigev. Etaub gefättigter (Whin. auf $2 \mathbb{3}$ olfs. milchblíttern)

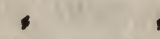

m mild)rabmfarbene, fóñffelformige \unf: te; Der Etaub braun

n balbenformige, weislichte \$lafelt, bie nach Der grof́fern Axe jerfiringen, und cimet faffeebraunen Staub aus/chütten (Wobr. Blátter ber Gartennelfe. LEriad). Eept. Dct.) *
E uphorbiae, SBolfsmiloffrabs pilf.

fcutellatum, ichú: felformiget.

caryophyllinum, शelfenftaubpilz.

o linis. 


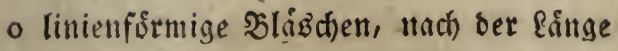
aufipringent, uno einen farmar;braumen Etaub ausichuittetio (WObn. Srasigals men und Grasulåtter)

lineare, linienforts miget.

p kuglig, weiflichtbraun; Die Sanmen ein

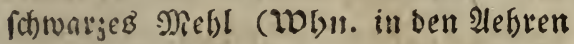
Der Gerffe, des 2 seizens, Mayfes, bet Şirfen, 万̧aberarten, einiger Birăfer, in Den Sllithen bes Socfabartä, unb Der Ecorjonere. Durch Sieinlidffeit, oder atch) Daburch verbutet man ibn, bafis inan mur jågrigen, moblgetrocfiteten Caamen baut) *

II. Iluter ber Erbe (Truffeln)

a mursellos

I แแนregelmåsitg, ftumpf, vielectig, bicht, wurjellos, fitwarzrindig (Wohn. in Wraldungen. Zieife Şerbit. Gebr. Epeife ,

2 nicbergebrickt, Euglig, faameni̊nnlid Tubcr, gemeine Tเuีfer. (Why1, unter Sroblblatter in Srsinters ungen, unter abgefallenem baum. laube)

$b$ angenurgelt,

Uftilago, Biand.

I fuglig, im Nittel meblig; (WDLn.

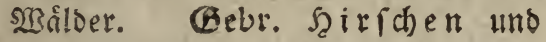

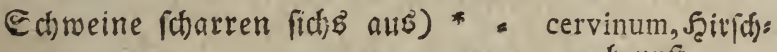

2 faft fugelformig, glatt, bidt), weidh biunfr.

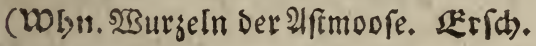
Epaitberbft)

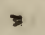

Lycopodium, Såulappe.

mufcorum, अ100: jetruffer.

a Die Aefre weitfdrueifig, nieberliegend; bie Slatter zerfitent, mit einem Szaare an ber Epite; Die 2lebren paarweife, gefielt

(20) 
(WWhn. Bugằnge ber Nabelgstger. Gebr.

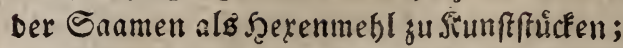
bie Pflanje zu Justeppid)en, und 23 einver, befíerungen) *

b bie Ilefte gablig;

clavatum, feulfors mige.

I bie Slitter zerftreut, bachiegelformig,

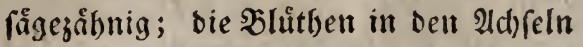
ber Blätter (LDhn. Oebirgmaloungen) * Selago, tanken.

2 brenfdineibig; bie Sblitter sufanmge: formige. madfen, vierfad) berumftelent, ange. orůfft, zugeipijt (WObn. $23 a l o u n g e n$.

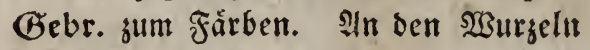
finbet fid) eine (Sochenille)

complanatum, vielgablige.

3 flad)gebrueft, friedsend, wie ber Stamm;

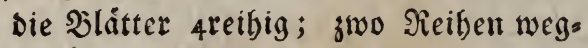
ftebéno, zwo die Spule (oben) Dachşies gelfơrmig Dectend; bie $\mathfrak{A}$ ehre fadenfơr mig, gablig (WOlnn. Saummurzeln) * radicans, mus c bie Reftchen mit abgefesten Jabrtrieben; zelinde.

bie Slưthenåtren eingeln, ftiellos; bie Slátter lanjetfơrmig in ber form eines Jưnfers im 23 ưffel um bas 2 efíchen ber:

um jerftreut (WDLn. SBälber. Bebr. jun (jelbfärben)

d bie Albren einjeln, blätterig, an Enbe ber annotinum, wachs boloerblatterige.

Alefte; bie Şlätter zerftreuet, mit Zähnen gefrant, fanzetformig Woly. (Jebirgmals Dungen)

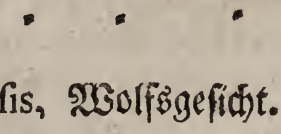

I Die şlåtter vollfommen gan; Der Sten. gel aufred)t; Die verblubten Relche en" formig, bangend (2)!bj. Suli)

pulla, fifivargtos ther. 


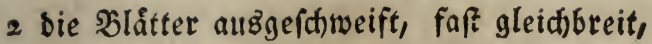
an ber Epitze lanzetförmig zulaufend, bie Slúthen und bie verblůbten Relche aufs

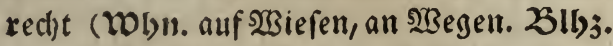
Jumi-2luguft. Gebr. Den Sdjweinen unb Sienen) *

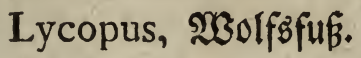

arvenfis, 2ldely rolfegeficit.

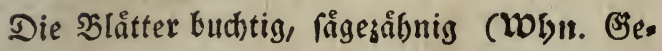
ftabe, Gräben, alte Nauern. Slly. Juni, Suli) *

\section{Lyfimachia, Evfimadfie.}

europaeus, eutos paififfet.

a Die Slůthen eingeln, aนş Den Blattwinfeln I ber Stengel nieberliegend; Die Slåtter enfơrmig, fpitigig (Woln. id)attige $\mathfrak{E a u b s}$

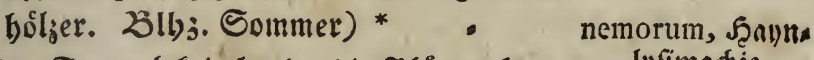

2 ber Stengel friechend; Die Slåtter bers: (1) fimadie. åhnlich), tellerfơrmig (Wobn. Orríben, Teiche, an Brichen. 23llyj. Sommer) * Nummularia,

b bie \$lluthen in furzen Irauben, an ben Pfenningftaut. Seiten ber Stengel auB Blattwinfeln, ges ftielt (Whn. Giríben. 25!h)3. Juli. Gebr. Den Sienen) - = " = thyrfiflora, ftraut:

c bie Sllithen in einer zufammgefełten, ris artige. fpenfơrmigen Traube, an ber Epitąe; bie Slatter 3 u. 4 fach), auch gegemúber (Wobn.

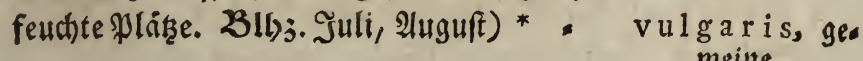

\section{Lythrum, \&ytfrum.}

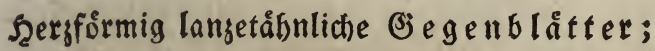
zmo̊lfmánnige blůthen in $\mathscr{A e h r e n ~ ( W W h r . ~}$ Geftade. 23lb3. Juli-Gept. Gebr. Fut: terfraut, zur (jerberlobe)

Salicaria, rothet

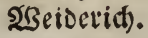
M. Malva, 


\section{M.}

\section{Malva, ঐappel.}

2 Der Stengel aufrect),

I raub; bie $\mathfrak{i l a t t e r ~ v i e l t h e i l i g , ~ r a u b ~ ( W W i r . ~}$ ungebaute, grasreiche \$lähęe. 25!bj. Juli, 2uguit) * = = . =

2 Erautartig; bie Slåtter 5-7lappig; bie Alcaea, Siega maistwur. Sappen fägejånnig, etwas fpişig; bie Stiele ber blátter und Sllithen bes baart; bie Slattanjąe enformig, ge. frant (Wobn. Adferraine, Felien, Naus. ern, Etrấent. Zlbj. wie sben. Gebr. Den $\mathfrak{B i e n e n ) ~ * ~}$

b ber Stengel mieberliegens; die Slatter fylveftris, Robis pappel. berzfoormig, tellerâtnlid), feid)t, 5-7lappig,

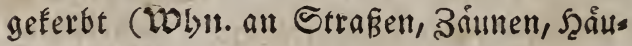
rern. 23lbj. Juni bis Sept. Gebr, mie obeil) *

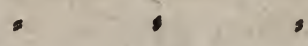

2Inmert. Tlfe 3 Irten baben eine ers rotundifolia, $\sin$ Tepapper. weidbenoe Rraft.

Marchantia, SNatc(jantie.

1. Der Slütbentnopf balkÉtgelfơrmig,

- I Drenlappig, (Woly. Matuern) =

2 fünffpaltig; ber slluttyenftiel ofne feld): triloba, oteylaps pige.

formige (Finfafiung (Whan. smifd)en

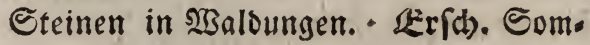
mer)

,

II. Der ß̧lutthenfnopf ftumpf, kegelforrmig, funffäherig (WWlnn. feuct)te, fduattige Stels

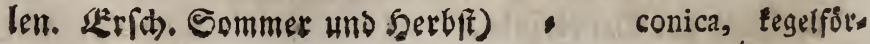
mige.

hemifphaerica, balbéugel fortmige. mige.

III. Det 
III. Der BluthenÉnopf flach, feidft act)tlappig; Der Bluthenffiel 4 fantig ( $\mathfrak{W b}$ h. verlaffene (Gartenbetten, auch) Mauern. (Rer (d). Mian)

IV. Der Shluthenfnopf fternformig getheilt; Der Slutbenftel runo (WDlun. an Duellen unb Sish)en swifhen Steinen, in (ih)attigen

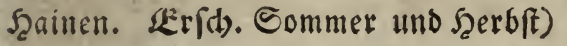

Marrubium, ânoorn.

u m be 11 a ta, Sil) it m mals dantie.

Itellata, fernfor: mige.

Die zehn Seldhłånne borftenågnlich, bafen: formig (Blbj. Juli, 2uguft. Gebr. Ger: berlobe)

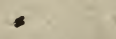

vulgare, weißer.

Marfilia, Marfilie.

Sierblatterig; bie Blätter berffơrmig, ůfers Sereus, an Der Epitee DeB Etengels (LDLu. Rachen und Síche) "
Matricaria, Nutterfraut.

quadrifolia, vievs blitterige.

I Die Blätter flach, zufammengerezt; Die Slatt(t)en enformig, zerfánitten; Die Sllis

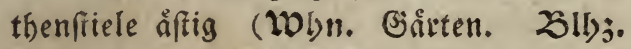
Juli ${ }^{*}=$ = = Parthenium, ges

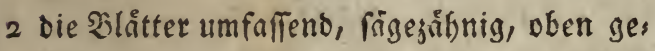
meines.

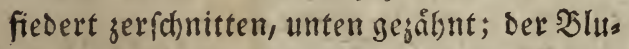
menftral am Orunde fatter gefơrbt (WDIn. 2lecker. 25lb). Juli)

fegetum, STHudjel:s blume.

3 bie Blatter boppelt gefiedert; bie Slått: chen fehr fhmal, 2-3ipaltig; Die Strufe lanjetformig (Won. 1t. Blb;. wie bben) * Chamomilla, (5)as

4 Die Sllitter alle ftiellos, langgezogen, faft mille. gleichbreit, grob fägesâthing; Der Etengel Iblüthig, aftlos; Der SRand ber Relchichups pen rdhwars (Woln. Gebirge. Zll)j. Juli (Sept.) tertéraut. 
5 bie Etengelblåtter ftiellos, långlicht, ges sånt, am Sirunde leberfórmig jerfonnitten; bie șurzelblätter geftielt, eyforrmig, geferbt; ber Etengel åtig; Der Ranb Der Sieldh: ichuppen vertrodfnet (Whin. an @traßen,

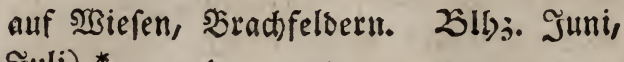
Iuli) *

\section{Medicago, Sichelflee.}

Leucanthemum, હ̧ånjeblume.

I Der Stengel aufretht; bie \$lutthen in Trauben, aufredt); bie Şuilfen in Edsraus. bengånge geroumben; bie Blátter lánglid)t,

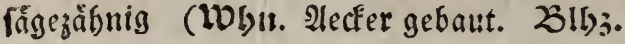
Juni, Juli. Gebr. ein febr gutes futters fraut) *

2 ber Etengel auffehent ; bie Slutthen trauls benformig; Die Relche gottig; Die Şúlfen fict)elfơrmig, vieliaamig; Die Silåttchen

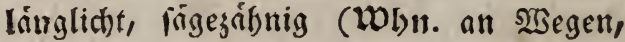

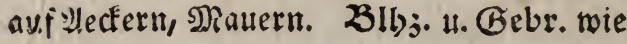
giben)*

- falcata, gemeiner:

3. Der Etengel nieberliegend; bie 2lebren eb)= fơrmig; Die Şúlien nierenformig, I paamig; bie Slo̊tter faft tellerformig, fägezåbnig

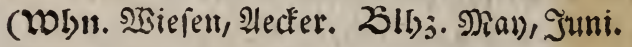
Gebr. wie oben) *

lupulina, Şor pfenficheltele.

Melampyrum, Sufweijen.

I Die Slưthen einfeitig,

a gepaart; Die untern Slátter volffommen ganj; bie $\mathfrak{b l u ̊ t h e n b l a ̊ t t e r ~}$

a gefiebert jerichnitten (WWhn. 2 Beiben, Borwálber, Laubbo̊lfer. Zibj.Mian2luguft. Gebr. Das Sindvieb fríft fie gerne) *

pratenfe, 2 Bieferts tubiveizen.

b gefies 
b gefiebert, gezåhnt; bie Rippen flafs feno (Wobn. Gebaue) - fylvaticum,

b ungeäbrt; Die Sllithenblåtter hersfor. $23 a l o k$ Eubeizen. Inig, gezignt; die oberften blittenlos, gefárbt; bie Reldye zottig (WWhn. Raub. bo̊lger)*

= nemorofum,

2 bie 2hehren vierfantig; bie Blüthenblótter blauter. berjfơrmig, gebrångt, bichtģånnig (IDbn. Gebuifche. 23!bs. Juni)

3 bie 2lebren Eegelformig, lang, weitlauftig; criftatum, framm: artiget.

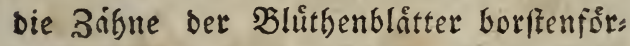

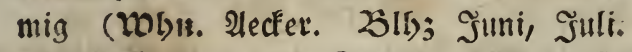
Gebr. Rinder und Schafe freffen fie gerne, fie bient zum Fårben, uno ben Bies nen; die Saamen geben §rod) * =

\section{Melica, Jerlgras.}

I Die åuñere Shlappe jeber untern Epelse nit Seibenbaaren fark gefrangt (WDIn. Jelien. Slbj. Man, Iuni)

ciliata, gefralty= tes.

2 bie Epelsen ungefrangt; sie slütben in arvenfe, $\mathfrak{Z}$ lefev: Eutroeigen. einer einfeitigen Tiaube; bie $\mathfrak{A e b r h e n ~ m i t ~}$

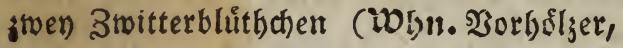
2luen. 23!b). Sommer)*

nutans, íberfan: gendes.

3 eine arme, einfeitige Sifpe; bie Seldse 2blütfig, bas eine $\mathfrak{b l u t t h c h e n ~ e i n ~ 3 m i t t e r , ~}$ Das andere gefaledtlog (LOLlt. Felien)

\section{Melilottos, Melilottos.}

I Die Şưlfen in einer Traube, maft, ůber: bangent, rumslidht, zrvev)faamig; ber Eten. gel aufred)t (Wonn. Aecfer. Blb;. Juni, Juli. Gebr. zum Futter, Flact), Fåt: ben) *

officinalis, gemeis net.

I 32 die 
2 bie Şånttchen bolbenfórmig, blåtterlos ; bie Slätter ftiellog, sfingerig, feinfeiben. glånzeno (WDln. fteinige Ctellfen) *

\section{Meliffa, Meliffe.}

Dorycnium, ges
fingerter.

I Die Sluthenftiele aus Den $2(h)$ feln, gablid)t,

långer alB Das frutzenende blatt - Nepeta, Birgs

2 bie Slutthen it Srauben, aus ben Slatt= melifie. winkeln, wirtelfórmig; die Bluthenftiele

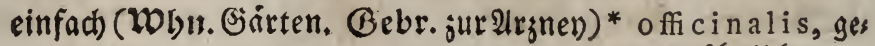

Melittis, Melittiö. iosfnilidje.

Die eingig befannte 2art wobnt auf gen, blitbt im Nay uno Juni

Mentha, Muinje.

melliffophyllum, gemeine.

I Die Slüthen in lainglidbten Aebren; bie Slätter friellog,

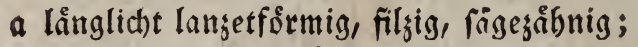
Die Ctaubfriden lónger als die Slume (WWhn. Griben, in Şedfen. 215j. Spats (ommer) * " " " fylveftris, Fof

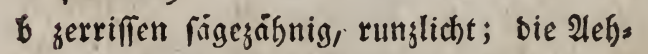
ren unterbrochen ( $2 \mathfrak{l b}$; wie oben) rotundifolia,

2 bie Sllutben in Dotirlen;

2 bie Duirlen geftielt;

a bie Slåtter eyfơrmig, gerunbet, faum geferbt; bie Tråger långer als bie Slumen; bie Stengel faft runo, fries

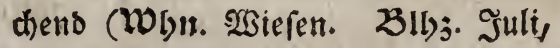
2uguft). , Pulegium, Polev:

$b$ folang als bie Slattficle; bie Relche borifig; bie unterften Slumenlappen Ianjetférmig; bie Staubfäben fo lang als bie \$lumenrógre (Błl)j. Suli, श(uguft) - = gentilis, Ëbets c bicht), 
( Did)t, ziemlid) glatt; bie Slatter evo fơrmig lanzetáfnlidy, meitlaulftig fäges zoifnig; Die Etaubföben lónger als Die Şlume; Der Slattftiel lónger als bie Quirle (Wobn. idhattige, fenchte (tellen)

- borftig, furffticlig; bie Slitter ev)= fativa, Binten: minzze. formig, weitlåuftig rảgejåbnig, et= was raub; die Etaubfáden lánget als bie Slume; bie Slattfitele fưr. zer als bie Duirle (WWhır. Gråven. 25l53. Juli-Eept.) * , = verticillata, quith

b bie Duirlen faft friellog, raub; die bluthige. Blåtter enfơrmig, weitlóuftig lágęăth= nig, rauth; die Staubfáben ungefingr von ber fringe ber Şlume (MVhur. Alecter. 23lly. wie oben) .* : - $\quad$ arvenfis, 2rkéer=

c bie legten Duirlen in Ropfdhen gefants muinge.

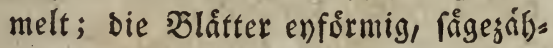
nig, geftielt;

a bie Staubfäben linger als bie Blume (Wolit. Geftade, Ditellen. Z3llj;. Sommer, $\mathfrak{h e r b f t ) * ~ = ~ " ~ a q u a t i c a , ~} 2$ tsoffer=

6 bie Staubfóden furryer als bie Slume minze. (Whln. Gârten. (Bebr. 2(rzmen) * Piperitis, Pfeffe: minge:

\section{Menyanthes, Bottenblume.}

Die Sllutten in Srauben, oreufad); bet ges meinfchaftliche כluetbenfiel fumpf, 3 fan. tig; bie Bläter 3 fingerig (WWlnt. See,

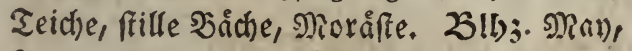
Juni. Gebr. ̧um รårben, Den Sienen)* trifoliata, 3blát: terige, Viberklee. 
Mercurialis, ß̧ingelfraut.

I Der Stengel gang einfach); bie Slátter ets was fteifborftig (Woln. Sergwålber. 2 lbj. 2lpril, $\mathfrak{M}_{\text {aiy) }}$ )

perennis, aubsaui

2 ber Stengel offtig; bie - Slätter glatt; bie einder. slůthen in Hebren (WWlyn. Bartenland, 3̊̊ume, Jૃecfen. 231l)j. Sommer) *

annua, gemeines.

\section{Merulius, 2 bernpilg.}

I Seberartig, mit ber untern flåche anges wachiell, bie obere runglicht, bơcfericht

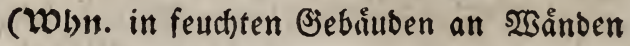
und an ber (Erbe)

Vaftator, verwùs

2 Etrunflos, faft bolsig, oben etwas filsig, ftender. ungleich, bofuferig, unten, ober an ben Seiten gewoebnlich mit åftigen, in einan: Der gefalungenen 3 lättern (Woln, an al. ten Brettern) ,

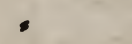

3 flachgebruidt, nieberliegend, aneinander ges fepiarius, 3nuns adernpily. wachlene, aus einem Nittel fommende, fid) allmählig erweiternde, am Ende zuges runbete Blåtter (WDbn. an Buchenftåm. men zmifhen গinde unb f̧ols)

lichenoides, fledji tenartiget.

\section{Mefpilus, Mipipel.}

\section{Unbeweldrt.}

I Die Blåtter langetfơrmig, unten filyig; bie blithen ftiellos, eingeln (Wobn. Gär. telt. 23lb3. Nay. Gebr. Die Fruidte zur Epeife; Sweige und faub zum Ger: ben, bas heols zu 2(rbeiten) *

2 bie Siläter eyformig,

a volffommen gang, unten filfig (Wolnt. Oebirge. 25lbj. Juni) . . Chamaemefpilus, niedriget. 


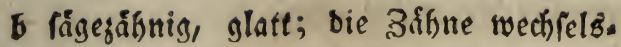
weife fleiner; bie Şlüthenbuindel fopf.

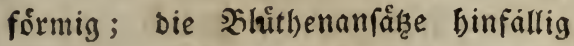
(W0la. H. 23lbs. wie oben)
Cotoneafter,

Milium, Scirjengras.

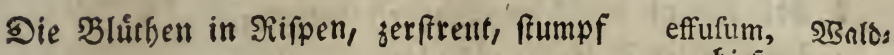

Moehringia, Mo̊fringie.

bitiengins.

Die einģig befannte Sirt wolnut an Felfen,

blibt in Suni

Molinia, Molinie.

mufcofa, Ferfen: móbringie.

I Die sifpengelenfe naft (Woln. feuchte פriefen. Zll;. Juli-Eept.)* $*$ varia, wanber:

2 bie siipengelenfe mit langen 3otten (WDIn. \$ergtbåler. 2ł!bj. Juni)
Mollia, Mollie.

I Die Etrimmdien felje furł,

a rafenbildent; ber Girund bes Sappelifte. les̉ unbebedt'; bie Slåtter långlicht, pitigig,

a mit einer Nittelribbe unb einen Scaare an Der Epişe, regftehent (WDIn. Mauern, Dâdher, Steine. Zleife Frůbling) . ' muralis, Mauet:

6 mit einer etwas úber bie Epize mollie.

Gervortrettenden baarlofen Nittels ribbe (WVl)n. auf fanbigem $\mathfrak{B}_{0}$ Dell. Zieife Sisinter)

- Die Saprelfitele aus ben Enben, eingeln; 2rund in acea, robrattige. Die Rapfeln pfriemenforrnig; bie SBläts ter långlidht, burd)rdeinig, mit einer Mittelribbe (Whin. Sitinen, jmifden niebrigem (irafe auf Thonboden)

fubulata, pfries menformige.
2 bie 
2 bie Ctåmmchen aufred)t, etwas ciftig,

a aufwairtz blätteriger; bet Rapfelbeckel lang, pfriemenfórmig, Erumm; bie Blîts ter lanzetfórmig, mit eines Mittelribbe (Holn. Sandboden)

b boh binauf mit 2 Surzelfaaren bebect'; unguiculata, vo: gelflauige.

bie sjlåter linienforrmig, langetål)nlid), mit einer Mittelribbe, im feuchten 3 แ.

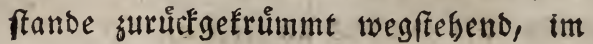
trockiten einmairts fraus (WOLn. be: waiferte STippen) * : = fallax, trúglidie.

c bie \$låtter breitlicht, sungenfơrmig, ber. ablaufend, mit einer rothen Mittels ribbe und einem wegfehenden fraare an Der Spike; Der Sirund Des Saplels fiteles beutlich) bebecft (WVht. Saum, wurjeln. Zieife Frůfling) = ruralis, baatblat

(2unm. ein Strobdach mit bierem Noo. terige.

fe bewachien, bålt romal långer, als obne baffelbe.)

3 bie trocfinen fruchtfitiele gewunben; bie

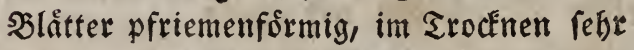
fraus; Die Rapieln faft waljenfórmig (Whin. W̉albungen. Zleife Spátfommer) tortuofa, gef́taus iete.

\section{Monotropa, Monotrope.}

Der Ctengel mebrblutthig (Wobn. Gid)ten: wurzeln. Zllj. Juni, Juli)* = Hypopythys, Montia, Montie.

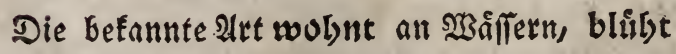

im Sav unb Juni $=\quad$ = fontana, ges

Morus, 


\section{Morus, Naulbeers.}

Die Slâtter herłfơrmig,

I und geigenformig, an ben Stielen breit, oben zugefpist, mit fleinen (sinfor)nitten verfebell, und auf benden Seiten glatt und glinjeno* : : = alba, weiß̈e.

2 unbeutlich 5lappig, frumpficht, am Salt De gesoifnt, uno beym Infuiblen bart uns fcharf* = $*:=$ = nigra, fofwarge.

3 zumeilen auch 5 ober 3lappig, am ?an: De icharf gezaignt, unten zottig; Die rriteden malgenformig* = , rubra, rothe. (Alle 3 strten werben bey) uns gego: gen, borsugiglid) aber bie erfte, Der, Seibenraupen wegen, weldhe, won Diefen Sláttern genaigrt, feinere Seibe macht. Die Fruidjte wer: ben gegefier. Das frol taugt zu Riaften, Fåffern uno Drebarbeit.)

\section{Mucilago, Nober.}

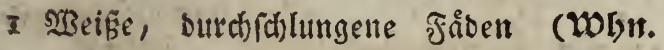
zwifd)en baummurzeln, zwilchen simbe

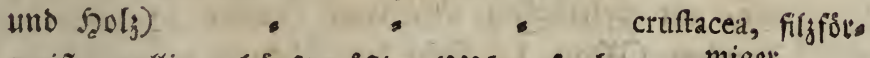

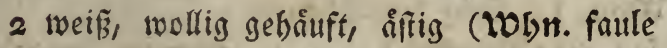
Silse, \$flangen unb thierifhe 2(usmürfe) plumofa, feset.

3 meifigtall, fpinnengewebartig (TObn. faus lenve Pflangen in verfaloffenen Drten) arenofa, fpinnen: 4 gallertige, an ben Eeiten oft mit Rugel, gewebartiget.s d)en befeste Fåben (Wbit. in şaffer ver: mefente Siorper)

\section{Mucor, Efjimmel.}

infuforia, 2 fufgu[x moser.

I. Geftielt.
A. Der Ctrunf einfad), wei B ;

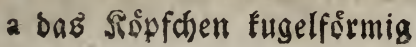


I twafferfirtbig, bann gruin, enblidi fchrwars (Whn. Broo, alte Epei. ren, faulenbe fruichte) : glancus, Obft:

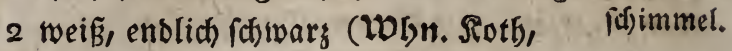
Das an feuthten Stellen vermes (et) = " . fimeti, Siothichim:

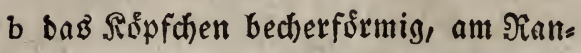
be zerriffen (WWlnn. faulenbe \$ilze) fungorum, Pilze B. tieffchwary, aiuferft flein, in ber IIt: fúsimmel. geno bas Rofpfchen in eine burchfichtige Situgel eingebúfft (WObn, unter

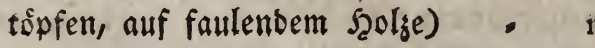

II. Stiellog,

microfcopicus, microliopifater.

\section{a fugelfớrnig,}

I weiß (WDitr. faulende $\mathfrak{B l a ̊ t t e r ) ~ a l ~ b u s , ~ w e i ß ̄ e r . ~}$

2 gelb (WOLn. auf Pflangen, bie unter Der \$reffe zu Siriuterfaumlungen zu feucht gebalten worden)

flavus, gelbet.

b gebåuft, gallertig, linfenfơrmig, zufams menfliefend (Woln. auf nafiem (jes máuer)

c Pefr ciftig; bie Aefte borftenfơrmig, fpar. rig, mit weitlåuftig ftebenden frudt). trauben (WWhn. Die Erbe ber Slumen.

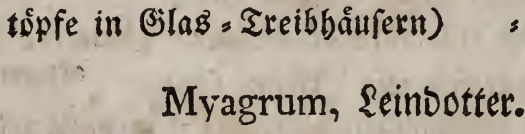

A. Das Schơttchen verfebrt evfơrmig,

a geftielt, vielfaamig; bie Blátter langet. ábnlich), pfeilfórmig (WOln. unter bem

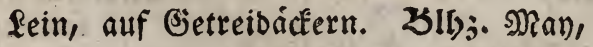
Itmi. Gebr. Der Saame zu gutem Dele, Futter ber Cingugogel, Naftung Der Siñne, Die Dlůtben Den Bienen)* fativum, gemeis net.

b etwas 
b etwas jufammgedruicft; Der Etengel ge. rifpet; bie Slátter linglicht, weitláufs tig fingezílnis (LVbr. Gebirggegenden. 2!l)3. Intmi, Juli)

\section{- faxatile,}

leinoottet.

B. Das Scho̊ttchen zufammgedruct fugels fơrmig, ztweofád)erig; bie Stengelblátter ungeftielt, pfeilförmig, rauh), bie untern

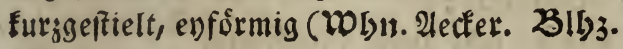
Nay, Juni)

Myofotis, Zergi(ßmeinnid)t.

paniculatum, ges rifpeter.

2 Die Caamen naft; bie SIattpitsen bicker

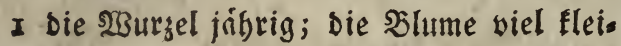
ner alB ben ber folgenden (WOHn. Aecker,

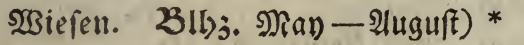

2 bie Srsurgel lang, aubbauternd (Wohn. Girás annua, einjåbrti ben, feudte $23 i e f e n$, Oebege, fable Etels len Der Alpen. 23!bj. Nay - Sctb.)* perenn is, auss

b bie Saamen mit bacfenformigen Eteifs Dauernoez. borften; bie Blâtter Ianjetfórmig "(WObn. an Etraffen. 23l!jj. Juni-2luguff) *

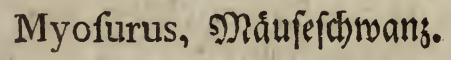
Lappula, flettens formiges.

Die befannte 2nt roobnt auf Ibonboden,

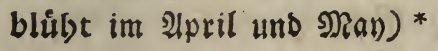

\section{Myrica, Bjagel.}

minimus, Eleiv ftet:

Strauchartig; Die Sllåtter verfebrt lametfớt. mig, fägegånnig, furggeftielt (LDhn. tročne Şeibenplåz̧e bergiger Gegenden. 23ll)j.

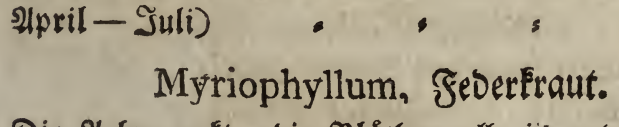

I Die 2lebre naft; bie Sllutben alle it ent: fernten, blattlofen Quirlen (Dol)n. Feiche,

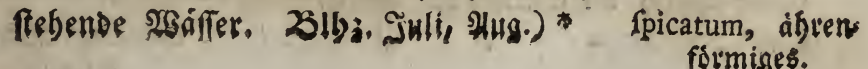


2 bie Bluthen in ben 2ldfeln ber Slätter ftiellos; fuinf slatter im Dutrle (WWIn. Gråben. 2̇lbj. Juli)* : $\quad$ = verticillatum,

\section{N.}

quirlfostmigez.

\section{Narciffus, श्रarciffe.}

A. Die Scheibe einblittbig;

a bie innere Stlume fefre furz, rabförnig, geterbt, trocken $=$ " poëticus, weise.

b bie inmere Slume glocfenfơrmig, Eraub, aufred)t, fo grof als bie niuf̧ere eys rumbe Slıme ", " Pfeudonarciffus,

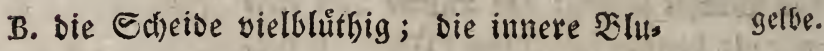

me glockenformig

a gefaltet, abgeftuţt, zmal fleiner als bie áuß̂ere; bie ஒlåtter flact)

Tazetta, ₹ngette.

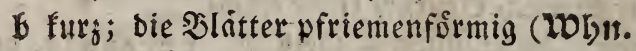

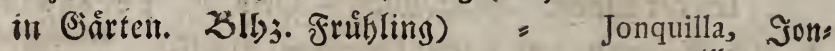

Narcuius, รืovftengraż.

Die Aebre alfrecht, borftenformig; bie $\mathfrak{b l u}=$ then einfeitig, in zwo Reiben (Woln. mas gere 2 Seiben. 2lly;. Juni) * = ftricta, ftartendeb.

Nekera, Nefere.

Die Rapfelu euformig; die Raprelftiele ges wunden, bis nn bie ફ̧âlfte mit ben Reld).

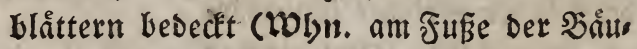
me in Nalbungen)
Nepeta, SRepte.

Die Blitben in unterbrodhenen 2lebren, quirls fơrmig, furzfitielig; Die Silntter geftielt,

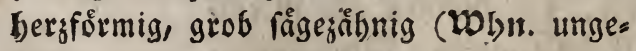
baute Drte, an Strafien. Zlly. Suli(Sept.)*

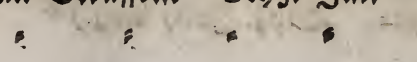
curtipendula, Eurzgeftielte.

Cataria, Saksen: múnze.

Nigella, 


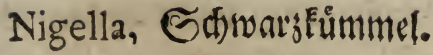

Mit 5 Stempeln.

I Die Rapfel birnformig, mit fefr langen f̧ơrnern; Die Şlumenblätter gang (WDln. 2ecker. Slbj. Juni-Dctb. Gebr. Den Sienen) *

- arvenfis, Felos

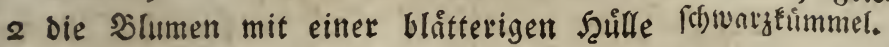
umgeben (Whan. Sairten) $=\Rightarrow$ damafcena, Damas

3 bie siapfeln rundlid)t, igelartigftacblicht; icenijchet.

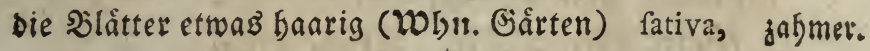

(2linir. Die Eaamen aller 3 Itrten find ges

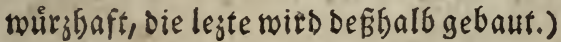

Nymphaea, Geerofe.

Die Slätter berzformig, gerundet, vollfom. men gans;

I ber Reld) bat rundlidte, boble, bicfe, inmenoig gelbe, glånzende, viel gro̊ßere Slatter als bie Slume (Woln. Seen, Seiche, Grriben. 25lbj. Juni, Juli. Gebr. zur Edheinsmaftung, zur હ๋er. berlobe) *

- $\quad$ s lutea, gelbe:

2 Der Melds) 4fpaltig, weif̧grůnlid)t; bie

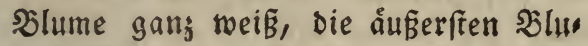

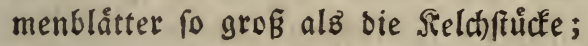
bie folgenden immer fleiner (WWhin.

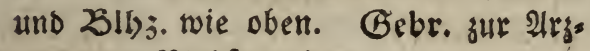
nen, zum Biegfutter) *

- alba, weífe.

\section{O.}

A. Stiellos,

Odtofpora, 2lettfaamen.

2 tellerfơrunig,

I sinnoberroth, randlos (WDbn. im úber.

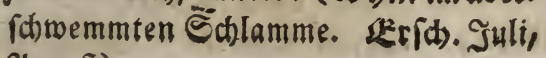
शैuguft)

im $\mathrm{m}$ arginata, tandlofer.

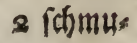




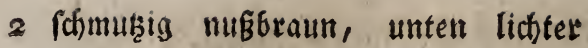
(Wobir. H. KErfh. wie oben)

applanata, fladjer:

3 gewelbt, rotb, mit fatrargen Fran. zen ant Ranbe (WWln. an ben Rain. Dern ber Fufitritte, meldhe Die Růhe auf lettigen 2 Beiben macher. Krifd. Sept.) $=$ = $=$ fcutellata, iffilo:

4 flad), alfentbalben glatt, meißggrau formiget. (IDln. zwifhen ben Etúcten ver:

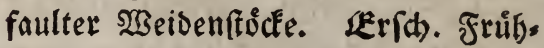
ling)

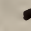

pallida, Glá̧er.

b bederformig, rotbgelb, am Rande feins jottig (WOLn. zmilchen Moogarten auf

- Thongrund. UErfh. Detb.) " lutea, getbet.

$c$ in ber Jugend fugelfórmig, im 2lter flach, inwendig mennigroth, uno glatt, austwentig braiunlichtroth, und allents balben ichwarjborftig (Wobn. faulende

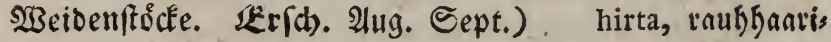

B. in ber Jugend furzgeftielt, fugelformig, get. grau; im Alter faft fitellog, faft fhuiffels fơrmig, \{chwarz, am Sande mit einem weifen, zerriffenen, aufrechten singe

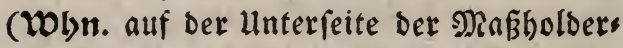

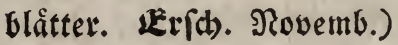

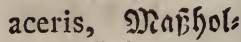
Detaditiamen.

C. faft Ereufelformig, braun; bie Minoung faum vertieft; Der Tellerrano fein ges ferbt (Wol)n. Stiele abgefallener Eichen. bläter. (Erfid. Octob.) - " epiphylla, Eichens

D. becherfórmig, gelb, inwendig glatt, auß̃en mit braunen פorften befest (WWhn. vers cottetes Pferbefotb. EErfd. Sept.) fetigera, bouftis get.

E. prise 
E. proifentirtellerfórmig, gelb, ourd)aแz glatt ; Der Schulffelrand glatt; ber Mittelraum in Der Jugend vertieft, im IIter flach (WDhn. jwichen bem Mioofe auf $\mathfrak{S u r}_{j}$ eln alter

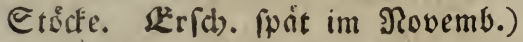

hypocraterifor-

F. seuvelbt, polftrig, braun; unten vertieft, weis̄lid)t, fơrnig, Deutlich) murzelnd ( 20 bn. Eanbboden)

\section{Ocymum, Sanfilien.} mis, prifentirtels lerfourmiget.

rhizophora, wuts zelnder.

Die Slatter evrumo,

I gefranjt, oder marsig, ooer blafig, oder glatt; Die Sielche mit feinen J̧aareat gefrangt

2 gans glattrandig

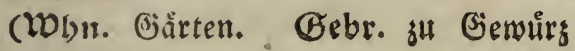
Bafilicum, ges meine. uno 2(izney.)

Oenanthe, Siebendolbe.

Ranfentreibend; Die Etengelbläter gefiebert, faft fadenformig rofbrig (Whrr. fiebende

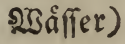

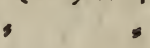

fiftulofa, to̊ 5 rige.

Oenothera, Silibrapunzel.

Die Slatter enähnlich lanjetforming glatt; Der Stengel rauh (Whar. Wfer, Gärten. 23!ba. Juli-Eept. Gebr. alB Eduunfens falat zur Epeife)* " " biennis, gelbe.

Onobrychis, Eiper.

Der Etengel geblittert; Die blüthenåhren langgeftielt; bie \$llumenfiủgel łleiner als ber Sield); Die frúlie frachlid)t (IVl)n.

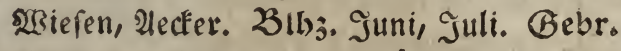
eines ber beften sutterftáuter, ben Şies nen) *

Sativa, gemeinet. 


\section{Ononis, Scaudjedfel.}

Die Blätter brenfingerig, bie obern einfad; Der Etengel nieberliegent;

I bie Aeffe fracblicht; bie Slittyen paar.

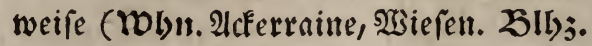
Julli. Gebr. Den $\subseteq$ dhafen und Bienen) * fpinofa, fecthende.

2 bie Aefte fatchellos; der Stengel flebrig

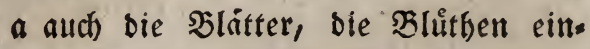

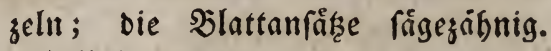
SSirflich eine eigene $2 \mathfrak{n t}$ ? ? , hybrida, $\mathfrak{B}$ aftat

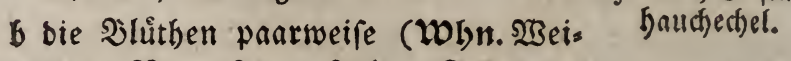
Den. 23lbj. Juni, Julli. Gebr. Das

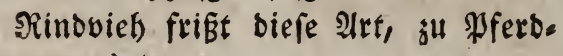
arzmen) *
Onopordum, Zellenfraut.

Die Blåtter långlicht enfơrmig, fpitigig, bol lss

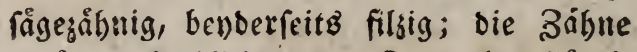
gezi̊nnt, faachlidft; Der Etengel geflugigelt (Whyn. an Straffen. 23lbj. Juni, Juli. Gebr. Die Eaamen geben Del, Die Blüs then bienen Den Sienen) * = Acanthium, weifo

Ophiogloffum, ఇattersuinglein. arvenfis, Xcters
baucffedjel. fę.

Der Stengel einblåtterig; bas Şlatt eyfơre mig, lanzetånnlich, ftumpf (WWbn. feuchte Wiejen)

\section{Orchis, Sinabenfraut.}

vulgatúm, beut idjer.

\section{Die $\mathfrak{S u r}$ zelfnollen ungetbeilt,}

I ein einzelner 23 uržllfnollen; zwen $\mathfrak{b l a ̊ t . ~}$ ter am Grunbe, und ein lanzettórmiges in Der Mitte bes fonft natten Stengels; Die untere $\mathfrak{B l u m e n l i p p e}$ 3ípaltig (201)n. in Gebirgtbơlern. Z Zlbj. Juni)*

Monorchis, $\$ B$ raminabentraut.

2 evfor 
2 enformig; die Unterlippe enformig, bey. DerfeitB einjåthnig, gan\}; Die ß̧látter

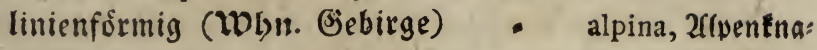

3 faft runb; ber Etengel blátterig; bie bentraut. Unterlippe fammetartig, ausgeranbet, grể, an ben Seiten außgeichroeift

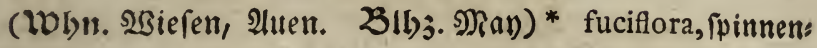

4 bie Unterlippe lanzetfơrmig, vollfommen fortmiges. ganj; baß f̧orn felgr lang; die Sei. tenblitter des Sieldies wegltehend ( 10 byt. in Scecten. Zlbj. Juni, Juli)* bifolia, weiß̄es.

5 bie Unterlippe 3ipaltig,

a zurưff́gebogen, geferbt, ober bas Mits telftucf auछgerandet; bas Şorn furs

a bie Seldjblîtter aneinander geneigt (WOLn. SBiefen. 25lbj. Juni)* coriophora, ftine $b$ bie Episen ber Reldhblatter fas tenoez. Denformig (WObn. Bebirge. Zlbj. Juli) - globofá, fugetblú,

b die fappen vollfommen gans; bas thiges. f̧orn fehr lang; die fieldhblátter

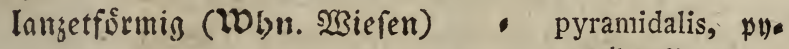
c bå Mittelftủc tief auṡgerandet; bag ramidenfỏrmiges. Scorn fehlt; bie 3 åufern field)blát= ter bicklicht, fumpf; bie 2 innern linienformig, funipf; bie $\mathfrak{L}_{\text {Gurgelfnols }}$ len långlid)t rund (20l)n. Alggrůnde. 23l)j. Juni) * =, = mufciflora, fie. 6 bie Unterlippe 4f̂altig; bas f̧orn ftumpf genblüthigez.

2 raub punftirt,

a bie Etưcfe fågegâlnnig, bie mittlern

fưrser; Die bllůtbenåbre febr fur

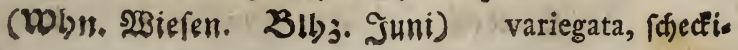
gis. 
S bie 3 oberften Relchblitter beutlid getrennt (Woltr. und 23lly. wie oben) *

b Die Stuicle faft gleidh, geferbt, ausz=

uftulata, punktiv tes. gefd)weift; Die Sield)blätter von ein= alloer entfernt (Whin. walbige \$erg, stefen. 23lb;. Nay)*

Morio, [aleptourz: netiges.

c bie zroeen mittlern fappen fleiner; Der Sippenrand fein geferbt, in ber Nitte gefintigter geflectit; bie zmen

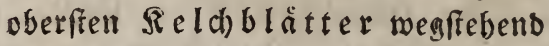
(WObn. Niefen. ZlI)j. May, Juni)* mafcula, ådftes.

7 bie Unterlippe 5theilig, raub punftirt; Das f̧orn ftumpf; Die Relchblátter am Grunde aneinander gerwad) fen (Wolnn.

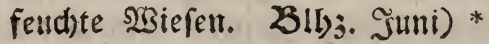

cinerea, graueb.

1I. Die SIUtrgelfnullen gertheilt, bandformig;

die lunterlippe mebr oder minder deut. (id) 3lappig

2 Daళ f̧orn Éegelforrmig;

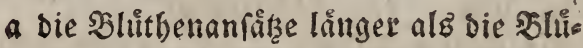
then; Der Stengel kebrig (Wohn.

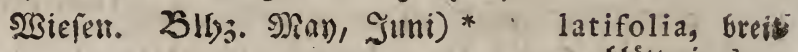

6 Die Slưthenanjątze fo lang als bie blaitterigez. Slůthen;

a bie Unterlippe enformig, geftreift;

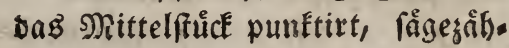
nig (Wohn. Serggegenden. Zlbj. wie oben) *

fambucina, Şots tundertinaben Etraut.

2 bie Ulnterlippe făgegábnig; bie obern Relch)blätter zurúcḱgebogen (WObn. S(Bálder)

- incarnata, fleiffos fîtbiges. 
b bas frorn frunm; bie llnterlippe eys forrnig, jutgerpitat; die \$blätter lintens

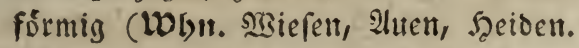
23t5; S. Juni) *

c bas f̧orn etwas fiurger als ber Frudyts

incarnata, woble riechendes.

fnoten; bie Seitenlappen ser Unter. lippe breit, geferbt, ber mittlere bolls fommen ganz; bie Relchblátter an ben Seiten wegftefeno (WOlnn. tmb Sllyj. wie oben)*

ג Das J̧⿻rn långer alB ber Fruchténoten, febr falmåchtig; zmev Sieldbblatter wegs

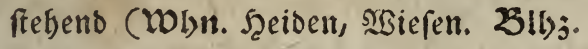
Juni, Juli) *

III. Das f̧orn febr furg (Stenbelmur;. Satyrium L.)

a bie Sisurgelfnollen stheilig; bie llnters lippe 3înaltig; Die Eeitenfuidfe lang, linienformig; bas mittlere febr furs, bie untern Blätter elfórmig (Woln. (Ses birge. Zlyj. Juni) . '

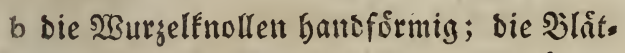
viridis, grůntirf)s ter linienformig; bie Aebre gebrångt; bie Slintter umgetebrt; bie Unterlippe ungetbeilt, eyfơrmig, fpirzig (WW 2libj. wie oben) " 3 maculata, geflect: tę.

conopfea, ftedf) fliegenbrittiges.

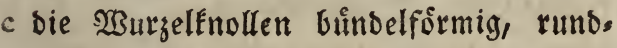
lid)t; bie fippe zipaltig; bie untern Slätter gerunbet, die sbern lanzetfor: mig (Whyn. wie oben. 23llj. 2itg.) albida, weiflichs ter.

Origanum, Majoran.

a Die Rebren gefairbt, runblid)t, in Siipen

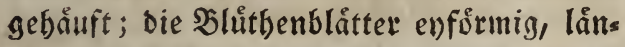
ger als bie Reldue; die Staubfáden linger 
als bie ร̧lume (WOLn. fteinigte Şegenbent, Etraffen. Blly. Ilug. Gebr. zur Arznev, in bie Siluche, zum frirben) * - vulgare, wilbet.

b bie $\mathscr{A e b r e n}$ runblidt), bid)t, etroas haarig; bie Slitter evrund, ftumpf (WDlın. Gåars

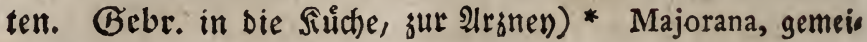
net.

\section{Ornithogalum, Jogelmild).}

1. Der Etengel ectig, zwenblatterig; bie

2. lutbenftiele in einer Dolde,

a einfach) (WObn. 2lecfer, 2luen. 23ll)j.

2tpril. Gebr. ben Bienen) * - 1u teum, gelbe.

b åftig (Molnt. Bll)b. u. Gebr. wie oben) minimum, f́leine.

II. Der Stengel waljenrund; bie Slütben

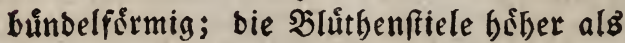
ber Sdhaft; bie Triger an ber Spitee auşgeranbet (WWln. Grasgårten, feuchte 2Biefen. 23lbj. 2(pril-Juni)* , umbellatum, Dok III. Die Blưthen ůberbangend, einfeitig; bie Denformige. Ctaubbeuteltråger bilben eine $\mathbb{G}$ lo (Whin. Graggirten. 25lbj. May)

\section{Ornithopus, Vogelfuß.}

nutans, libethadn. gende.

Die Blitter gefiedert, die $\mathfrak{B l a ̊ t t c h e n ~ z a b l r e i d ) ; ~}$ bie Shullen bogenformig (WWhn. an Strafs (en. 2lls. May)
Orobanche, Sonnenwurs.

perpufillus, होleis net.

E Der Siengel gaits einfact), fammetartig; bie Slétbenblåtter lanjetfórmig (WDhn. Gebuifche, Dbifgoirten, aud) auf Angern, meiftens aber auf ben 2 surzeln perenniren. Der Wflangen (a)marozend. Z3!bj. Nây, Juni) $*$ : $=$ = major, gemeine.

2 Der Etengel aftig; bie \$lútben sfpaltig (3)bj. Epåtfommer) - : ramofa, åftige.

Orobus, 
Orobus, כiergerbfe.

Die Blätter gefiedert; die $\mathfrak{b l a ̊ t t c h e n ~ e y f o r e r m i g , ~}$

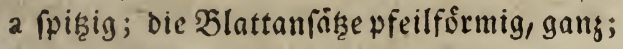

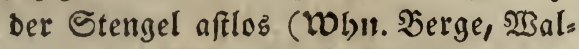

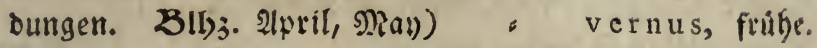

b mit einer Giranne (6 paar Blättchen)

Die Bluithen faft einfeitig; ber Eten=

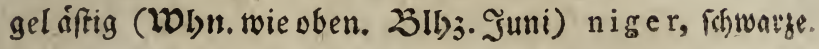

Osmunda, Traubenfarren.

Die $\mathfrak{b l a ̈ t t e r ~ g e f i e b e r t , ~ b i e ~} \mathfrak{S l i t t c h e n ~ m o n d f o ̈ r . ~}$ mig; Der sllutthenfiel eine Sortfeţung bes

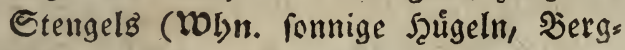

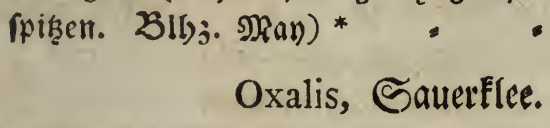

I Der Scijaft einbluttfig; Die blätter breys

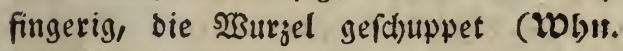

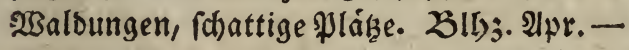
Juni. Gebr. bie Blåttchen an bie Fles cten ber Sleider und ber Seinwand ans gerieben, und bie angeriebene grüne Farbe wieder ausgewaifhen, tilgen bie Glecten fo gut, als bag Sals biefer $\mathfrak{P f l a n g e}$ * Acetofella, gemeis

2 ber Stengel âftig; bie Sllutbenftiele mit Lunaria, monos blätteriger. Dolben; bie Slatter wect)pelfeitig (MWhn. Gartenlant. 23ly;. Aluguft. Gebr. ̧ut Epeife; bem Sieb angenebm und gefund) corniculata, gels ber.

\section{P.}

Paeonia, Jdonie.

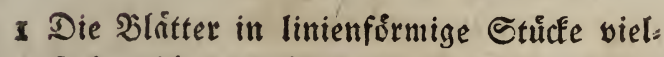
fac) geridgnitten (WObn. um 2lerbing. Slbj. 2)(ugu(f)

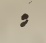

?
, net.

$$
\text { ler. }
$$




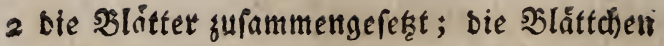
loinglid)t (HWlan. in Sirirten. Zlbj. Som.

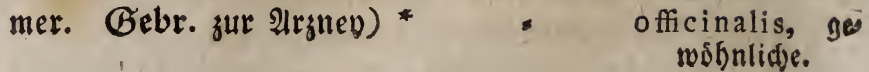

\section{Panicum, ভேjraben.}

I. Die 2lehren walsenfórmig,

a ftâtig; aus bem Grunbe ber 2ebrohen

I báufige lange Borften; bie Eaamen wellenformig, rumglid)t (WOLn. Alecfer, (b) a tenlanb. 23lbj. Durd)gebends Juni) * . - glaucum, grauer.

2 lange Sorften; bie Caamen nervig

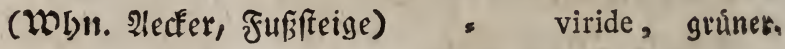

b an Grunbe einigemal unterbrodhen; vier Slutt chen an einer Iraube; grobe bor ften am Birunse ber bionlglein, långer

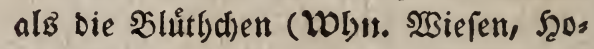
pfengårten)

II. Die Aefree långlidft, fufammgefe\}t; bie verticillatum, quitlfömiget, Alebrchen fnaulfírmig; bie Sluethden mit Sorften untermisht; bie Stielden raub) (Whli. zwar fremb, wirb aber mit bem Şir(e gemein(d)aftlid) gebaut) * - italicum, wel(j)er:

III. Die Aebren wechelsweife, und paak weife an ber gemeinfthaftlichen Epuble; bie Aebrchen abermal in fleinere getheilt; fteife Şaare an Grunte ber Slüthenfticle und ben Slappen ber Эölglein; bie bålgs Iein mit Sirannen (Woly. fetter Boden)*

IV. Die Aefren wedjelfeitig an Der Epise Crus galli, bahi nenfubartiget. DeB Szalmes, fabenformig; bie Epuble

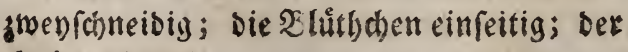
Salm idwach

,

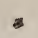

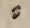

fanguinale, $\mathfrak{T l u t s}$ [chwaden. 
V. Die Iefren bolbenformig gefingert, fas Denformig; bie Slutthen einfeitig; bie

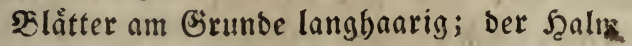
nieberliegent; bie Ranfen rundlid)t, trie: chent

VI. Siself umber flatternbe siifpen; fteifs dactylon, gefin. getrer.

baarige Slattidheiden; bie Sâlglein zuge. rpitst, nervig (Woln. wirb auf Aecfer ges baut. Gebr. veridhieben jur Speife)

miliaceum, șitre, Btein.

\section{Papaver, Mofn.}

a Die Sapfelin glatt,

I linglid)t; Die Sorften bes Stengels ane

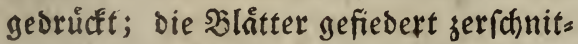
ten; Die Stride eingefdritten (WDLnn. 2ecfer. ¿lb3. Juli) - - dubium, zweifers

2 faft Eugelformig; bie Sieldhe glatt; bie baftet: Blåtter umfaffend, eingef(h)nitten (WObn. in allen Giårten. Gebr. Die Saamen geben gutes Sel, Das zum Vrennen beffer taugt, als Baumól; bie Delflls d)en bienen bem $23 i e$; fonft bat biefe Pllange bumn und follåfrig mad)ende Sirífte) *

fomniferum, (5ati

3 fuglidht; ber Ctengel bebaart, sielbli: tenmohn.

thig; bie Slåtter gefiebert gerfdonitten; bie Slättchen eingefdnitten (WDln!. 2lecfer. Blb;. Suli. Gebt. giebt eine rotbe farbe) * $\quad=$, Rhoeas, rothe

b Die Rapfeln raub, feulenförmig; ber Eten Rornblume, Sitaps gel blätterig, vielblútbig

Parietaria, (S)lasfraut. pertofe.

Argemone, feulen: fortmiget,

Die Blåtter enfơrnig, langetåbnlid), fpişig, nechjelfeitig; Die Seldhe zwendaltterig officinalis, officis neller. 


\section{Paris, Einbeere.}

Man fent bishter nur eine Art (MOlnn. faubbofljer. 25lb;. Juni, Juli. Gebr. zum Fårben und zur Niniaturfarbe) * quadrifolia, viel" blåtterige.

\section{Parnailia, Einblatt.}

Man fennt nur eine Art (Wobn. feudite 25iefen. 2ll);. Juli-Sept.)* ${ }^{*}$ paluftris, Sumpf (3tnm. Die Staubgefáfe fint wanbelnb.) einblatt.

\section{Paftinaca, ઝaftinacE.}

Die Slätter gefiebert; bie Bláttchen enför = mig; bas ungleiche brenlappig (WDlyn.

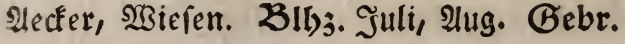
Die $23 u r z e l n$ jur Epeife)* - . fativa, gemeiret.

(2lnm. Der Gartenpaftinact unteriaheis Det fich vom milben nur baburd), baf̈ ber zabme glatte, ber wilbe aber raube Slítter bat.)

\section{Pedicularis, \&åufefraut.}

a Der Etengel áftig; bie ઝlåtter gefiebert; Die Blattchen gezánnt; bie Reldhe an ben sionbern fnorrig; bie Şelme ftumpf (Whun. feuchte 23 iefen. Zlbj. Nay, Juni)*

b Der Etengel am Grrunbe åftig; bie Reldh. paluftris, อ $u m y f:$ láujeftraut.

- rinber glatt; Die blätter langetformig, gefiebert zerfhnitten; bie Stücke gezåbnt, bie Slůthenblätter geơlbrt (Woln. Siebirge. 23ils;. Juni)

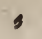

c ber Stengel faft aftlos; bie Slumenbelme fpişig gefdhnabelt; bie Reldie etwas bebaart (20l)r. Gebirge. Z3!bj. Juli) - fylvatica, $\mathfrak{x a l d :}$ láuléfraut.

roftrata, finnabel: blitthigez.. 
d ber Stengel einfach;

I bie blithen mechfelsmeife, uns orev. fach); bie Relche fpitzig geferbt; bie Blättchen gefiebert zeridynitten, bie Etuicfe gerundet, geferbt (TOLn. șals Dungen)
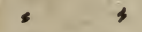

Sceptrum, ifitives

2 Die Bluthen in blatterigen 2lebren; bie Difober Zepter.

Bluithenblátter feld)lang; Der Reld) fünf. zånnig, fdiefmuintig; bie Şelme ziem. - lid) ftumpf, an ber Epitze etwas zuruick: gebogen (Wolnn. Giebirge. Slly. Juni) tuberofa, Enolfio

3 bie Aebre georångt, mit untermengten ger. Ṡlâttern; ber f̧elm wollig; bie un. tern Blatter boppelt gefiedert; bie ober. fien berablaufeno gefiedert (WOlnt. uno 3lbj. wie oben)

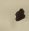

comora, zopfiges.

\section{Peplis, ગુepliş.}

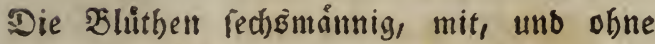

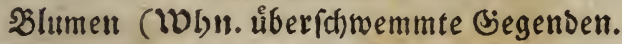
251b:. 24เguft)

$\checkmark$

Peucedanum, Scaarftrang.
Portula, eutos páifóde.

a Die blátter 5 mal orentbeilig; Die \$lâtt. denl linienfirmig, lang, vollfommen gans

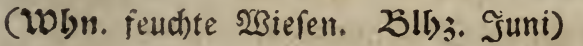

b bie \$latter gefiebert zertheilt; bie Igeile officinalis, officis neller. lanzetformis; gegenúber, an ber Spite mit emem furzen Etachel (Whn. fette 2 siefen. 23lb). Juni-Sept.)

Sil a us, Cilau.

c bie untern Slátter brenmal, bie obern zmen: mal gefiebert; bie lezten 3 lättchen 3-4lap= pig; bie Blåttctjenlappen lanģetfórmig, am Rande gans, an ber Epitze furzftad)=

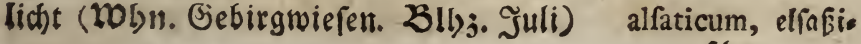
fdoet.

Peziza, 


\section{Peziza, Secherpils.}

a Sed)erfirmig,

a mit linfenfo̊rmigen Siơnern angefưlt,

I inmensig geftreift, glatt, auben zottig (Woln. all alten 2 rettern) - hirfuta, rauber.

2 benderfeits glatt (WWhr. an faulen Stơcten)
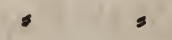

lentifera, glattel.

b oben mit einer tellerågulicjen, weiñen, (d)warzpunftirten flîdhe (Wobn. auf

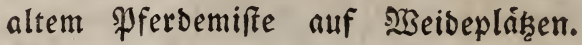
(Exrid). im Sommer)

3

-

b napfäbnlid), Fråufelfơrmig, bỏutig, brăıน,

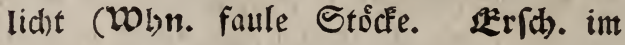
Sommer nad) Siegenwetter)

c żweifelfafte $\mathfrak{b e c h e r p i l}$ e;

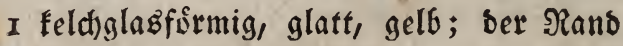
ftumpf, aufgeric)tet (WDbu. an ber Erbe. (2Erid). im 2 (pril)

2 frunflos, in ber Jugeno fuglid)t, glos cyathoides, mapf, ábnlicbet.

punctata, puntetit: ter.

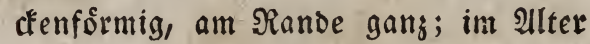
erweitert glocfenformig, am Siande zer:

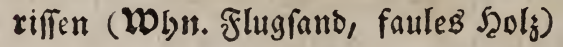

\section{Phalaris, Bjlanjgrab.}

arenaria, Gansi bedjetpily.

I Die Rippe faft eyrunb, und ábrenfưrmig;

- Die Epelsentlappen fdifformig (WDLntr. miro gebaut. Gebr. ber Eaamen fir bie $\mathfrak{E a}=$ nartenvogel, giebt aber auch gutes \$iebl zu 2jrod) *

canarienfis, Canas rienjanmen.

2 Die গiipe walzenformig, hibrenåbnlich, glatt, ichmal, frumpf (WObn. Siaine, SBiefen, Etraffen. 23!bj. Juni, Juli) ${ }^{*}=\begin{gathered}\text { phleoides, riefth. } \\ \text { grabartiges. }\end{gathered}$ 


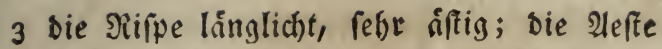
aufrecht, am Girunde jeber Epeljenflappe

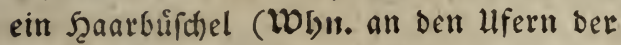

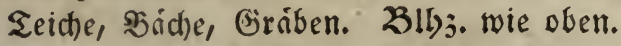
Gebr. Futtergraß *

\section{Phallus, SNotd)el.}

arundinacea, rober attiges.

a Dhne Enfaut; Der feut faltig, enfơrmig naft,

I an ber Epiţe fegelfơrmig, unten vom

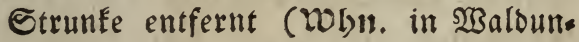
gen unter ben Såumen. (Kerid). im Frůbling) = " patulus, offre.

2 unten am Etrunfe angemadiren (WObn. Doftgairten, Eaubbo̊lzer. Kerfit). im Fribling) * - $\quad$ efculentus, efss

b ber StrunE aus einer (Enbaut; die Epite Deg f̧utes offen (WDLn. SSaloungen) impudicus, ftin Phafcum, Shnmuno. Eenoe.

I Die Slåtter enfôrmig, zugefpiţt, etwas megftebend, die oberften angeichlofien (Wolyn. Gartenland, 2leder, Nodererbe. KErid. mit Srapfeln im Scornung, und bann wies ber im 2uguff) * = = = cufpidatum, fuges

2 bie Blåtter lanżtấnlid) linienfờntig, şiem= fpijtet.

lic) megftebent; die obern aus eirem breitlichten Grumbe pfriemenförmig (WWln. auf fandigem Ibone an (d)attigen, feuct). ten Etellen. Kapfeln im Juli) " fubulatum, pfites

3 bie ßlåtter langetfírmig, volffommen gang; menfortmiger. am Stamme flein, entfernt; um bie frúchte lang, pfriemenformig fpitigig, gebrangt, im

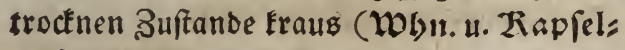
xeife mit Den Früblingsbungerblúmchen) crifpum, gektaus feter. 
4 féfr flein; bie Şlåtbre pfriemenfórmig langetrifnnlich, aufrecht (IDbn. feuchte Stel. (en)

nitidum, glanzen Det.

Phafeolus, Shifole, Soofnen.

I Der Stengel fich mindent; Die Slumen ftehen pantweife in Trauben; die Decf. bléttchen Fleiner als bie Sieldhblätter; bie รૃůlfen berabbangent * = - vulgaris,gemeine.

2 Der Stengel aufredt, fich nidst mindend, glatt; Die Deckblattchen grốfer als bie Reld)blätter; Die Scuillen berabbangeno, zufammengebruofft, runglid)t (Wobn. bevde Arten fino in Gå̊ten gemeit. Gebr. รur Speife)*

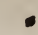

nanus, 3̧werg bog: nen.

Phellandrium, Jferofaamen.

1 Die Slätter zmal gefiebert; bie Abtheilum. gen won ber Scauptribbe unter rectiten unb ftumpfen $23 i n f e l n$ wegftebend; Die lezten Şlätd)en vielfach zerfd)nitten (Woln. fillle (3ewåtier. 23!lj. Juli)

2 bet Etengel faft blattlos, jiemlich einfad); aquaticum, gemeis nel.

- Die Slátter boppelt gefiebert, eingeid)nit= ten; Die Stůcke febr fpiscig lanjetfơrmig; bie Dolbe roth (Woln. Sobgebirge. Wll); Juni, Juli. Gebr. eines der beften Futs terf́råuter)

,

(1)

Mutellina, Mats tau.

\section{Philadelphus, Sironenjafmin.}

Die Slátter gezåant (WDbn. in Şecken milb, in Girten Des G̈eruches regen. 20lb3. nit $\mathfrak{A n f a n g}$ bes Sommers. Gebr. Den Sienen) *. 
Phleum, Eiejhigrag.

a Die Relche furjgrannig; Die 2lebre wal. zenformig, lang;

3 Der Şalm aufrect; bie खsurgel einfach

(robn. 23iefen. 2blbj. Nay-Sept.

(Gebr. Futter) * $\quad$ - pratenfe, gemeis

2 am Girunde unfrudtbar; ber Salm aufs febeno; bieşurzel bulbenfơrmig ( $\mathfrak{W}$ )n.

$2 \mathfrak{b}$ j. น. Gebr. wie oben)* = nodofum, Enotis

(211m. nur eine 2 bart ber vorigen?) gez.

- die Relche behaart; Die benben Girannen fo lang (oft linger) als bie fielchtlappen; bie 2lebre enformig, malsenábrlid) (HOLn. allentbalben auf 2ltpen. Zlbj. Juni, Iulli)

Phyfalis, Jubenfirfde.

alpinum, 2fipene liejchgrab.

Die Slâtter paarmeife aus einer Stelle, gang zugefpigt; Der Stengel frautartig, am Grunde etwas áftig (WWhn. verfachies ben, auth in Giårten. Zll;. Juni-Aus

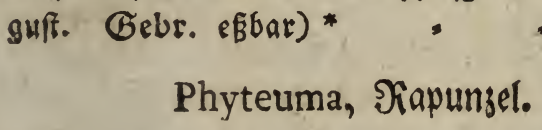

Der Stengel einfact); Die Slîtter fágejåh. nig, Die untern geftielt,

1 berffórmig; Die 2lebre långlid)t die Narben zwentheilig (WDIn. Ratatige Grashugeln. 2lbs Juni, ฐ̃uli. Gebr. bie Şlütben Den Bienen, Die \$\$urzeln zur Spcije) *

2 bie Şauptd)en faft Galbfugelfo̊rmig; bie Alkekengi, geo meine.

fpicata, langaifs. rige.

ঐarben orentheilig (WDLn. Gebirgtuáls Der. B!bj. Juni)

- orbicularis, fteigs runde.

Pimpi- 


\section{Pimpinella, Sibernelle.}

I Die Sllittchen Der 23 urgelblïtter enformig, bie Der Stengelblätter lanjetformig, alle eingerd)nitten (LDhn. magere wseiben, Etraffen. 25lbj. Juli-Eept.) * , Saxifraga, Hleine.

2 ber Stengel ectio; bie Slâtter enformig, ecfig eingefanitten, bas ungleidue breylap. pig (MVhn. feuchte $23 i e f e n$, Giebirge. 2615:. Juni, Juli)

3 bie Slâtter boppelt gefiebert; bie Slättchen magna, grofie. ber joten Dromung tief eingefdnitten; bie

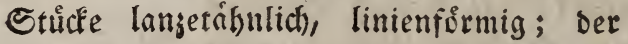
Stengelectig(10hn. Scobgebirg. 23!b3. Juni) diffecta, ferfdnits

Pinguicula, Settfraut. tene.

I Das f̧orn ber Şlume rpikgig, fo lang als

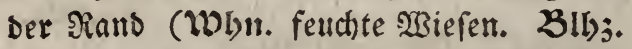
May) * Die Blume blau - vulgaris, gemeis

2 Das f̧orn ber Slume fitumf, furryer als nes.

ber Siand (Whir. allf Illpen allentbalben,

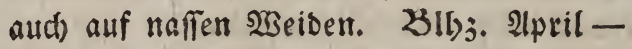
Juni) * Die Zlıme toeif

Pinus, Sidate, Foofre, Tanne. alpina, 2flpenfetts Etaut.

I Die Nabeln paarmeife aus einer Stheibe; bie erftern cinfad) (WDGn. in STaloungen úberall.

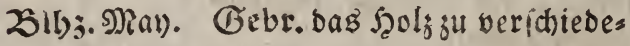
nem Gebralche, giebt audh) Dech, etmas Ter:

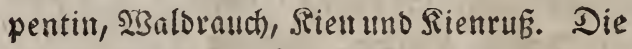
Bluttben liefern båufigen $23 a$ chsftof) *

(2tum. auf Dem Bebirge giebt es eine 2rt, Deren 2efte nieberliegent, uns fylveftris, Fifice, Sitefer = Siens Mantelbaum. linget als ber Stamm find, und bie man Listibeli, Lecterten, puinilio beišt. Das . helz Davon giebt vors treflidse Sd)miebetoblen, uno ba Sirummbolsofl.) 
2 funf glatte Nabeln aus einer Scheide (20bur. Gebirge. Bebr. Die Sürbelnuiffe fino fomadibaft zu effer, uno geben ein fachones Del)

-'

3 bie গabeln buifdelmeife aus einer Stelle,

Cembra, 3uitbels nแถ์ abfallens; bie Sapfenid)uppen evförmig, am Siande zerriften (WWln. auf Giebirgen båufig, aber auch in andern Gegenben, meiftens angepflangt. Gebr. Daß̧ ḩols vor allen Sånmen vorgůglich gut, ftark, Dauerbaft, befonders zu S\$affergebåuben,

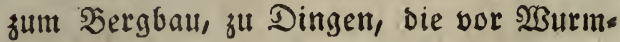
fraßz fidjer feun follen, fu febr guten Siobs. len. IUs Dem Safte madyt man febr fd)one weike frandfhuthe. Die Borke bient zum Bjerben. Der venetianifhe Serpentin fommt von biefem Saume, befs

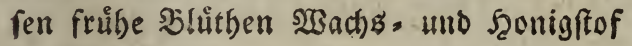
(iefern)

4. Die Slåtter eingeln,

Larix, Eerchen: baum.

$a$ an ber Epizze eingefdnitten; Die Zapfen linglid)t (WOln. 2 baloungen. Gebr. gu Şecfen, giebt Serpentin, Beigentbars, Roblen) *

Abies, Edettanne.

6 pfriemenformig, etwas ftechens, in gwo Reiben; Die Zapfen långlid)t; Die Echupen am Sande wellenformig uno zerriffen (WObn. $25 a l$ bungen. 25!h). פial. Gebr. giebt gutes Baubols, Wech, Terpentin, Oeigenbark, Dient zu Gerăthid)aften, Diufitinftrumenten; Die Sinde sient sum Gierben; Die నmeige Dienen ร̧น \$randtmeiii)*

$\therefore$

Picex, Foth. tanne. 


\section{Pifum, Erbje.}

Die Slattfticle rumblid)t; die Slattanfíze unten zugerunbet, geferbt;

I bie Shlutbenftiele mebrblutthig (WDgn. Aecfer, Giårten. 2̇lb;. Juni, Juli. Gebr. in bie אuchche)* . . fativum, gemeine.

(2nm. Die Slluthen Der Felierbre weifi, aud roth, pantreife zer. freut. Die rotbblutenden Erb. fen, und jene, beren Stengel auf ber $2 i s u r g e l$ trodfen gemorden, wer. Den nicht weich.)

2 bie Shlithenfiele einbluthig (WDbn. มecfer. Zlbs. 2uguft) *

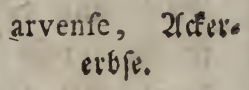

Plantago, 2regerid.

a Der Edaft blattlos;

a bie Sllitter enformig,

I fiebennervig, geftielt; die 2lefre fehr lang (20bur. an Etraffen uno Şån. fern. Zly). Man bis Sept. Gebr. Der Snamen zum futter Der Cings voigel * : : = major, grofer.

2 furzgeftielt, etwas foisis, fommetat. tig; Der Echaft rumb; Die Alefre ebformig, oder walgenfơrmig (Whn.

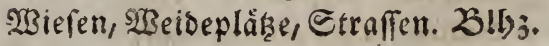
Sommer)*

6) bie Slåtter lanzetfórmig,

media, Steitues gerid).

I idjmal, bebaart; bie 2lebre långlid)t, (d) war (Whn. Biebirge und hoh lie, gende biegenden. 23lbj. ManIMIi) *

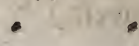

alpina, 2llpentwe gerid). 
2 Die Rehre enforrmig, naft; ber Ed)aft

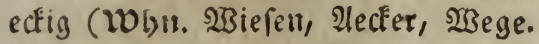
23l53. 2tpril bis Eept.)* - lanceolata, Epis,

b ber Etengel ăfitig, frautartig; Die ßlåtter suegeriof. linienforrmig, etmas gezäbnt; Die slútben. bäuptchen blattlos

\section{Poa, Nifpengras.}

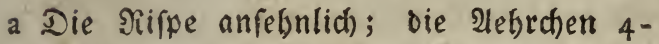
6blithig, gleich)beit (10)n. Biråben. 23153. Juli. Gebr. gutes Futter, uno auf Nid. râften mit Dortbeil zu bauen) - aquatica, $23 a f f e t s$

b Die Rifpe unanfebnlid); bie Aebrdjen 3rifpengiaz. 8blutbig, am Enve abgeftoflen; Die wilnern Epelzentlappen enformig, am Grumbe unb an ben Råndern von Şaaren glinjent; bie innern gefrangt (IDbu. Gebirge. 23!l)3. Juni)

c Die Sippe fpammenlang; die Alebrhen fies Pfyllium,
jaamen. benblutthig, linicnfơntig; bie bålglein felgr ftumpf; Der Salm unten etwas fries d)eno (2315;. Juni)

fálina, Satzrifpen: gเaต.

d bie Riipe furr, ziemlid) enge; Die Aefr. alpina, 2lpen: rifpengraz. d)en 2-4blutthig; bie Epuble furghanarig; bie ăufern Epelgenflappen langetformig, am Brunde wollig (WDhll. an 2 segen,

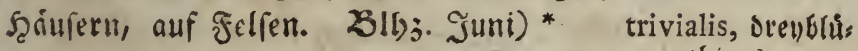

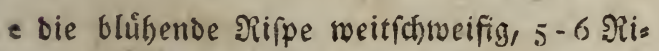
thiges. ipentifte aus eben berielben Stelle; bie 2llebrdten 3-5blittbig;

I Die Epuble und Epelgen bebaart;

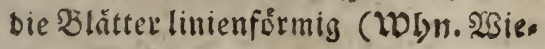
ren. Ż!bj, Juni)* - anguft if o lia, (i)malblíttetiges. 
2 enformig; bie Enelzen am Grunbe mollig; bie Silatter jiemlid) breit

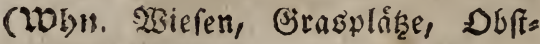
górten. 2̇ll)j. Juni. Gebs. ein gus teE Futtergrab) *

f ber Şalm etwas jufammgebrůfft; Die SRi: pratenfis, $\mathfrak{i s i e j e n ;}$ rifpenglas.

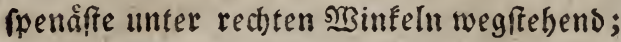
bie Epelgentapten naft (Wobn. Srsiejen, 2lenger, an f̧åufern. Zll)j. Fruibling, Commer. Gelir. wirb swar vom Vieb gers ne gefrefler, bleibt aber şiemlich niebrig) * arnua, jäbriges.

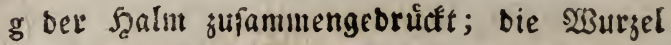
Eriechend; Die \$luthenftiele der Эiifpe ein. zeln; bie $\mathscr{A e h r d h e n ~ v i e r b l u t t h i g ; ~ b i e ~ c i u s ~}$

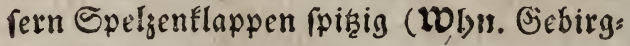
gegeriden. 23ib3. Juni)

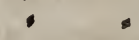

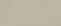

h ber Şalm gefrummt, (ct)mach; die Rifpe enge, uiberbangeno, mit aufgerid)teten $\Re$ i. ipenaffen; Die Mebrchen 2-3blutbis (BDbn.

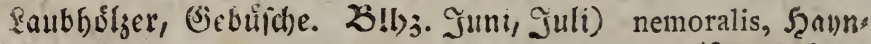
i bie Rifpe cinfeitig, zienlid) ausgebreitet; die Aebrchen walzenformig, vierblinthig; die äunern Epelgentlappen fpişig; Die sisurget bulbenformig (Wobl. Bebirge.

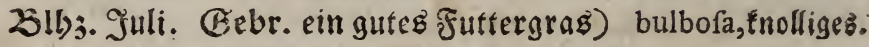

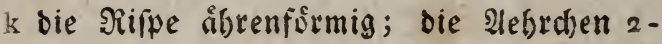

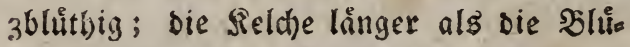
thenftieldhen; Die Bålglein uns Spelgen id)arfipizizig (Wolnn. bergige 2lecter, গabel.

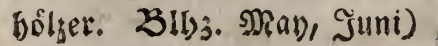

Polemonium, Sriegs์blume.

criftata, fremmfors miges.

compreffa, zus fammigedrúdtes. tippengrab. 


\section{Pollichia, Jollichie.}

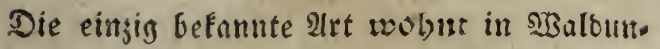
gen und 2tuen, blübt in Juli, 2uguf *

Polycnemum, Sinorpelfraut.

Galcobidolon, gulbe.

Es if nur eine 2 at befannt

Polygala, Sireusblume.

arvente, 2feters fnotpelfuat.

a Die Şlutben federbujhfơrmig, in Trauben;

I ber Etengel Erautartig, einfad), unten niederliegeno; bie Slätter ourdjaus fothal lanzetfơrmig (LOLn. Graspláţe.

3!b). Nav, Juni. (Gebr. Den Bienen)* vulgaris, gemeitte

2 bie Etengel fiemlich) aufred)t; bie uns tern blátter gro̊ser, werílibrt eyfósmig (พoln. u. Slb. wie oben) * , a mara, bittere.

b bie Blưtgen cinfad) zerftreut; bie Epitze bes Schiffchens gerumbet; Der Stengel firauchartig; bie Slåtter linglicht evfírs mig (Whit. ḩeiben, Esebirge. 251 ): 2tpril-Sept.) * : Chamaebuxus,

Polygonum, 2rsegtritt, నudiweigen. buxbuátterige.

A. Der Stengel gang einfach, eintigrig;

a Die Aehre walgenformig; bie Blatter enformig, oun blattifticle herablaufeno

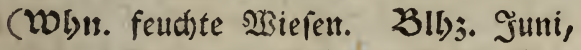
Juli. Gebr. bie Blåtter zu Gemůre; Die Srangel giebt Mebl gul brod, uno sient zum Gerben; die Şlüthen bienen Sen Sienen)*, , Biftorta, Iinttel"

$b$ bie Alebre bindfabenformig; bie obern เพนI'. Slátter lanjetformig (WDi)n. bof lies

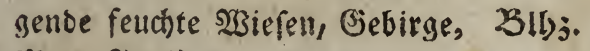
Miai), Juni) * 
B. bie Blutthen fünfminnig, zmeen Ģriffel; bie Staubfåben långer als bie Sielchblåt.

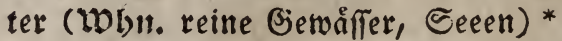

a m phibium,

C. bie Slưthen fechşmånnig; bie blåtter lan. शुed) jelwegtritt. zetfơrmig,

a nur etmen, aber tiefgefpaltenen Giriffel; Die Slîtter glatt; Die blattanlăke ge. franzt (WObn. Aecler. 23lly. Sommer,

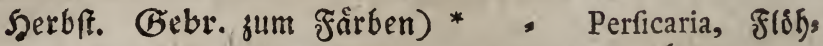
b zween Giriffel; bie Slattanfąhe franzen= Eraut.

log (Why. Geråben, Teid)e, Cecen. 2315. Juli, 2lıguft) *

Hydropiper, $25 a F_{5}$

D. bie Blúthen ad)tmånnig, z̧wentweibig; bie ferpfeffer. Slåtter lanzetformig; ber Etengel fraut. artig, nieberliegend (Wobu. Etraffen, Fußs: wege, Alecter, an Scålfern. Zill). Som= mer, Şerbft. Gebr. Die Samen geben gutes Mehl) *

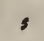

E. bie Slätter hersformig; Der Stengel fid minbent;

a bie Blůthen fiemlid flach; bie Reldy.

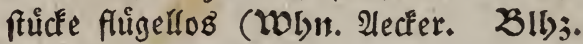
Suli, 2uguft. Gebr. Der Saamen, wie ber vom $\mathfrak{z}$ udweizen)

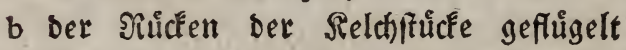

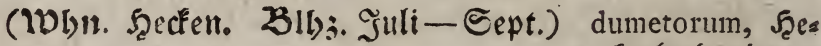

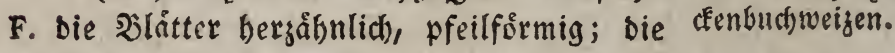
Etengel ziemlid) aufredt; bie Ranten ter Saamen zabnlos (Wobn. Alecker, wo er meis ftens gebaut wirb. Zlb;. wie oben. Gebr.

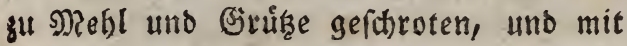
gleidgiel haber vermengt giebt er eit vors treftiches $\mathfrak{d}$ frofutter. Die Shlutben eine wortrefiche Sienenweibe. Die Snamen wers ben auth von Dogeln febr gerne gefreffer) * F a g o p y r m,

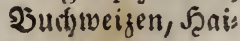
Den. Poly- 


\section{Polypodium, Tipfelfarth.}

I Das SIaft gefiebert gerfchuitten; bie Studfe fumpf langetforemig, wed)felsweife, in eins altoer verflefieno; bie sisuzel formppis (Whn. 23aldungen) * " " vulgare, Engels

2 Das Slatt gefiebert; Die Silattchen etmas fichelfiormig, fägejălinig, fradhlid) gefranjt, am vbert Grunbe mit eimem Blattolye (Whir. Scobgebirge)

3 bas Slatt fait boppelt gefiedert;

a Die erfien Blattchen lanjetformig, paak, weife am flevigen Strunte frent, uno alt ifm mit ben unterfien blátictien. filucten ein Biered bilbend (WObn. Thal, oungen)
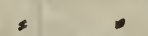

b Langetfórmig; die legten ßláttd)en etwas Phegopteris, 23us d)entüpfelfartn.

funmpf, fharf eingeid)nitten; Die \$latt: ribbe glatt (Whn. an Selfengueflen) fontanum, Suelle,

4 bas Slatt boppelt gefiesert; bie Blättchen

Lonchitis, Ditif Exnilt.

\section{Der jwoten Dromumg}

a gefiebert serfditten,

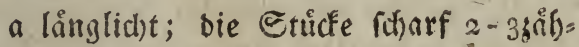
nig (Whyr. 2Balomingen)

dentatum, ge Jåñ

b Iangetfơrmig; bie Etidle $2-4$ mal ein tǜfelfartn. geichnitten

c giemlid) entfernt, wie bie ber erften crenatum, gefere. tet. Sronung; Die Ethide fumpf geferbt; Dav Blatt lanjetformig

b in eitunder verfiefient, tel.

a löinglicht, reid)t gef́erbt, sugerunoet; Der Etrunf flenin

= Tilix mas, Farrn. flautmannlein. 
b ppişig langetfórmig, fågełåfnig, am obern ङ̈runde faft geơbrt; bie Zెanne mit einem Stachel; ber Strunt mit Rlenen befezt

e enformig, fachlid)t gefranzt; bie ber erften Soronung etwas fichelforrmig aculeatum, ftachs

5 bas গlatt brenmal gefiedert; bie Slâtt. fpinofum, feinfors friger. dhen ber lezten Drbnung gefiedert zer. fchnittell, feinftachlicht Iid)ter.

6 baB $\mathfrak{b l a t t ~ m i t ~ g e f t i e l t e n , ~ r e c h t w i n f l i d ) t ~ g e s ~}$ criftatum, famm: fórmiget. genůberftehenben ร̧låttchen; bie Blättchen boppelt gefiebert, bie ber jwoten Dromung wed)felfeitig, gerunbet, in einander vers fließend (WDhn. 2 Baldungen)

\section{-}

\section{Polytrichum, भSiderthon.}

1. Die Rappe haarlog; die Rapfel malsen,

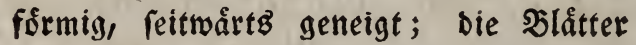
lanzetfórmig, fägezâfnig, gewellt

II. Die Rappe befaart; bie Siapfel
Dryopteris, Eis. dentupfelfartm.

a rundlicht felchforermig; bie Blåtter bicks lidht, langetfortmig, gang s nanum, Eleiner,

b aufred)t, walsenformig; bie Sefbarung unterbrochen; bie Silatter nabelformig, am Girunbe mit einem båntigen Rande, bidflidt; bie unterften febr furs harcynicum, c twalzenforrmig; ber Frudtffiel oben ges Searzwaldoswidets nunben, Der Decfel niebergebruidkt, mit einem fegelformigen Sthuabel " aloides, aloeblåt. d vierfantig; ber Stamm einfad); bie teriget. Slitter linienfơrmig, lanzetåbnlich

$\mathfrak{a}$ in ein fraar auglaufend - pilofum, Gaariget.

b baarlog,

I fågez̊n̊nig

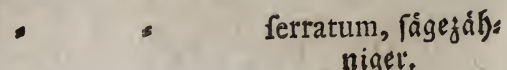

niget. 
2 faft ftectjens, fpięig, gans. commune, gemcis (MDIn. 2Baldungen. Die Siapich net. reifen theils in Fruthling, theils im Spatherbfte.)

\section{Populus, ঐnppel, Expe.}

a Die ミlåtter faft tellerfórmig, fpiąig, ectig,

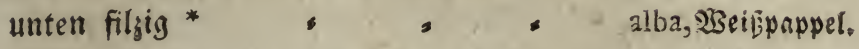

b bie Slåtter tellerformig, benderfeitz glatt, gejâfunt winflicbt * - t tremula, Elpe.

c bie Slätter faft Deltafơrmiz, roeitlåufig

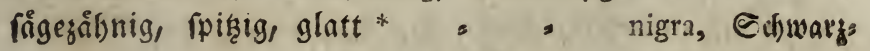

(TOb)r. Iuen, Scecfen, an Straffen. parpel.

Slbj. Niárz, 2lpril. Gebr. Das

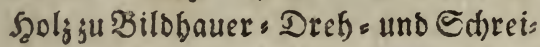
nerarbeiten; bie Ssolle taunt ffatt Baummolle.)

\section{Portulaca, ઝoortulack.}

Die Blatter feilfòrmig, gerunbet; bie $\mathfrak{b l u =}$ then ftiellog (Wobn. Gairten. 23lbj. Jull, 2lug. Gebr. Solat)* = oleracea, gemei. $_{\text {net. }}$

\section{Potamogeton, Eaamfraut.}

I. Die Slåtter fitellog,
2 berjformig, umfaffend * o = perfoliatum,
b lânglicht, gefpiz̧, burchidheinig, mersis, neşourmig geabert * - lucens, ourdd
nigez.
c gegenuiber,

I enformig Lanjetäbnlich, brennervig, an Der Epise megitebeno : denfum, dititbláts

2 lanjetåbnlich, etwas wellenfórmig, teriges. paarweife von einanber entfernt ferratum, fägefors: migę.
55
d gegen. 
d gegenúber, unb wedhelpeitig, linienforte mig,

I am Grunbe wegftehend, bann aufrecht (Dem Etengel paraleil); ber Sten. ge! rundlicht - - pufillum, fleines.

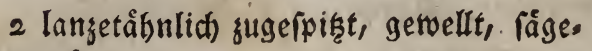
zoihnig

e wechfelfeitig *

crifpum, fraule: blätteriger.

I breitlicht linienfo̊rmig, feinnersig, gu= geipint; bie Aebren etwas weitlăus tig; ber Stengel gablicht

-

2 linienformig, lang; bie 2lebre lang weitliuftig unterbrochen*

II. Die ßilatter geffielt, nervig, eyfórmig, fdimimmeno*

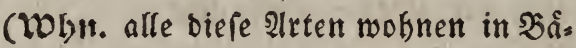
d)en, Gráben, Seeen, Ieidhen; blĭ: ben im Juli uno 2luguft. Gebr. ifle Saamen werben won Enten ges fuctit.)

\section{Potentilla, Jotentille.}

2 Der Etengel ranfend; bie Blatter gefie. Dert; bie Slåttchen rägezáfnig (WOLn. Bieh. weiben, Etraffen. 25lbj. Nay - Suli.

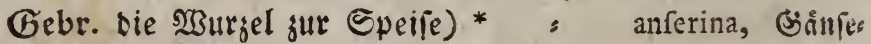

b ber Etengel aufrecht; ridy.

a bie Slatter funffingerig; bie Slåttden

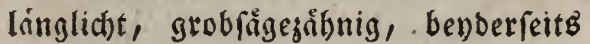
bebaart

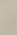
recta, nufredte.

$\checkmark$ bie Slatter fünffingerig; bie $\mathfrak{b l a ̈ t t ( t ) e n ~}$ feilfurmig, eingeichnitten, unten filsig (Whir. Idferraine, Etraffen. 25lh).

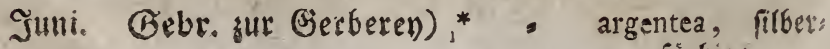
fárbigt. 
c sielblithig; bie Şlumenblâtter lảitg. licht; Die Fruchtbsten befjant; bie Slitter finffingerig, an Der Spine mit anliegenben Bảbnen jügejágnig (WObu. zwiid)en Felien. 20lb3. May) " caulefcens, vielo

c ber Etengel ziemlich aufred)t, ober nicber, blüthige. geworfen auffelgent; bie Slatter gefin: gert, ungeftielt (Wobur. trodfue Grinde. 25li)j. Eommer und Şerbft. Gebr. zum (jerben)*

d bie Etengel nieberliegent;

Tormentilla, 3/utruig.

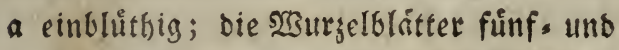
fiebenfingerig; bie Slättchen feilfơrnig, am Borberenbe

I pharf eingefdinitten; Die Stengels blatter brevfingerig (Wobn. ma=

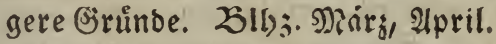
Gebr. Den Bienen) *

2 fígezäbnig, bebaart; bie Stengel: verna, Frůblings: potentille. blitter 3-5fingerig (Wvbur. ma=

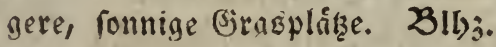
Mara, 2lpril. Gebr. Den Bies nen) *

6 vielolutt)ig;

I ber Blutherosen bebart; bie Blats ter fünfingerig, am গorberende mit alleinanter liegenden 3ifnen

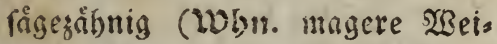
Den. S1b): Mray, Stmi) * alba, weíbe.

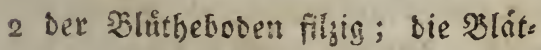
ter funf= brenfingexig, an Enve gisabut, am sianoe peibenábnlich gefrangt (Wohn. Gebirge. S\$l)j. Sikgufit)

clufiana, clufilde. 
c gablid)t; bie Slatter gefiebert, bie obers

ften brenfingerig (Woln. magere Brun.

De. $3(1) j$. Niay) * $=$ - fupina, liegende.

e ber Stengel friechent; bie blåtter gefin. gert (WDln. Wsalfergráben, feuchte, thos

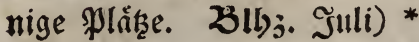

Poterium, Nedierblume.

reptans, ftries chende.

unbewehrt; bie Stengel etwas ecfig (Wolnt.

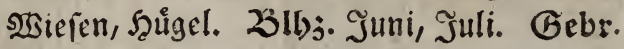
(Gerbereb) *

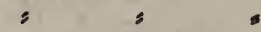

Prenanthes, Şafenftraudf.
Sanguiforba, ges meine.

Soinf Slúthdhen in einem Reldhe.

I Die blătter lanzetfơrmig, grob̧åănig

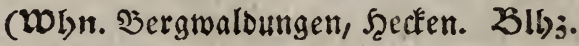
Suli, Siuguft)

2 Die Slåtter gefiedert zerfhnitten, bas ånßerfte Stůck orenectig, orebipaltig

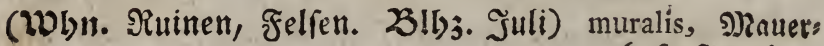
Primula, ๔d)lufelblume. purpurea, tothet. bafenftrautí.

1. Der Sdhaft armblưtbig; Die Slluthen alle ůberhangend; ber Sllumenrand aufgerid)= tet; ber Schlund glockenforrmig, aufgetries ben; Die Sliatter runglid)t, gezaibnt, oben glatt, unten fammetartig (WObn. SWiefen. Zlly3. April, Nay)

II. Der Schaft vielblưtbig;

hybrida, $\mathfrak{b}$ aftentos

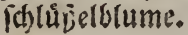

a alle Slüthen úberbangenb; ber SIlumen=

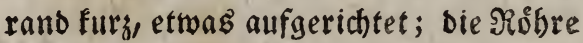
in Der Mitte etwas aufgeblalen; bie Blïtter rumglidst, gezăhnt, unten bes baart, oben glatt (Whlu. B̋årten, હiras.

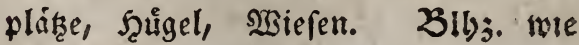
oben. Bebr. Den bienen) * " officinalis, ges wosfntide. 
6 nur bie åußern Sllutthen ůberbangend, bie unnern aufrecht; Der blumenrand flach wegftehent; bie sjlåtter runglicht,

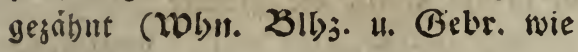
obcin)*

,

,

c alle Slumen aufrect);

I ber Sllumenranb flach wegftebenb; Die Silátter faft glatt, geterbt, untelt meblig weiß (IDbn. naffe sisiefen, an (jtáben. Zlbj. Niay-Juli) * farinofa, nueblige.

2 Die Sllîtter frigejåbnig, glatt, ver. fehrt enfirmig; Der Echaft etwas långer als bie blâttcr (WWblr. auf Gebirgen und in Bointen. 25l5j. Shărs, Ipril, im Giebirge Juni. Gebr. zur Sierde, und den bienen)* Auricula, 2furis felin.

\section{Prunella, ßrunelle.}

2. Die Slitter Durchaus eyforrmig;

I bie Slume noch einmal fo lang als ber

relch (Wobrr. fetter boden, an Giráben.

25153. Juni-Sept.) ${ }^{*} .$, vulgaris,gemeine.

2 bie Slume 4 mal fo lang als ber Rel(t)

(Mbn. Dưre \$låke. 25lb). Eommer) * grandiflora, grof,

b bie untern $\mathfrak{B}$ ! i̊ter långlid)t evfơrmig, gea blumige. ftielt, bie vier oberften linienformig, ges fiebert, gezübnt

\section{Prunus, פfflumen.}

x Die Slüthen in Irauben; bie Slätter an Der Rúckeite Des Birunbes mit zmo Drů. fen (Woln. Şecten. 25ll)j. Nian. Gebr. bie Früchte ếbar, Das şols ju eingelegten Arbeiten) *

Padus, elirens beetie.

\section{lole.}


2 bie $\mathfrak{B l u t t h e n}$ in Dolben,

A. faft friellog; die Slåtter evformig, lan.

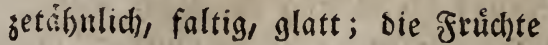

a grof́

a fdreargroth, faftreid), fauter.

$b$ roth, etwaz feft, fauer - 2t maxellen.

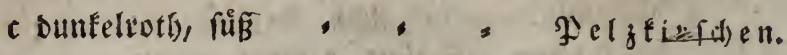

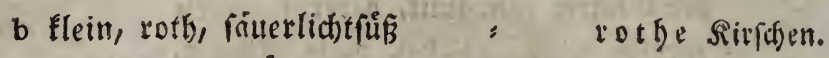
(Woln. Dbfigairten. 23lly. May.

Gebr. Die Frúchte ef̣uar.) *

B. ungeftielt; bie silnitter lanjetăhnlid), auf ber Ficffeite behaart (Whn. 2ors

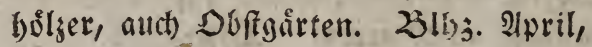

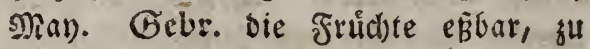
Srandtwein)

3 bie shitbenficle meiftens eingeln, furg; avium, Ecintwars Eiticide.

bie Blitter langetähnlid), enfớrnig, oben gefurcht, unten aberig (Oebr. Die vers fidirbenen Arten von Frudten find ein fehr fdimacfbafteg, gefundes Doft. Die Rerne afler sirfoch = und Pfiamenarten geben gus

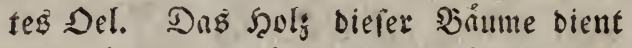
jum Sărben, vorzúglich zu Geråtbfd)aften, bie bet freben Suft nicht ausgefegt fint)* d o meft i c a,

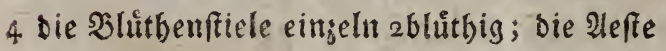

furzf(tad)lid)t; bie Şlatter eyrund, am Siante gezabme, auf ber untern Fläche nol. lig, einwălts gerolit (WWhn. (Jårten) * infititia, Stichen.

5 bie Blithenfitele I und 26 lutbig; bie 2lefte ftadidlid)t; Die Slatter glatt langetfơrmig

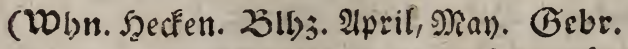
Die Rinbe uno bie unteifen fruidte fók ben, unb geben eille gute fdrwarge Dinte. Eejtere bienen audh sum Sdjlebenwein) * fpinofa, Eibleben. Pteris, 


\section{Pteris, Saumfarm.}

Die Slátter orenfach bufammengefest; bie Iezten Etricfe gerunbet lanzetfơrmig, voll. fommen ganj (Wobn. Nadelbưlzet. Gebr.

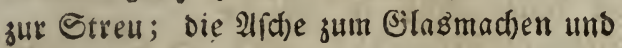

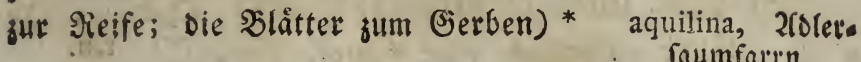

\section{Pulmonaria, \&ungenfraut.}

Dié șurgelblitter enfơrmig, bergåthnlich, raub (Woln. Eaubbulzer. Bibj. Man. Gebr. Den Sienten)*

-

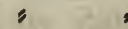

Pyrethrum, NGundfarrn.

Die Blätter gefiedert; Die SIlätchen gefies Dert zerfontten; Die Studfe fägezåhnig; Die Shlutben in einem Stranße (WObn. 2orgólger. ふら!bj. Juli, 2uguft) - corymbiferum, frrauß̧ต์

\section{Pyrola, MISintergriin.}

I Die Staubfäben auffebeno; Der Griffel abrårtż gefrúmmt; Die Sllåtter tellerfơr. mig (WDIn. \&aubbơlger. 23lbj. Juli)

2 Der Siriffel gerabe; Die Staubfäben gegen rotund ifolia, tundolătteriges. Denielben gebogen; die Blätter tellerförs mig, runo geferbt; bie slúthen jerftreut (Wobn. Bebirgwaloungen. \lbj. Juli) minor, tleineres.

3 bie Traube einfeitig; Die Slátter fpişig enformig (WObn. WSaldungen) : fecunda, einfeiti

4 bie Blưthen boldenforrmig geftellt - umbellata, ioldens

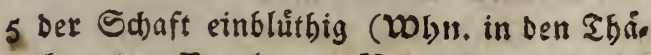
ttagendes. Lern Der Boralpen. \$!l)3. Juni, Juli) uniflora, einfiน, thige.

Pyrus, 


\section{Pyrus, Sirnbaum.}

- Die Slåtter enfớrnig, ftumpf, volffommett

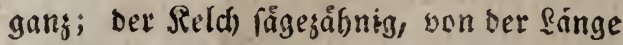
Der Şlume; Die ふllúthen cinzeln (WWhn. an bet Donau wild, fonft in Oårten. 2jlbj. Nay. Gebr. Die fructit jur Epeife)* Cydonia, Suits

2 bie blótter evfformig, fehr ftuntpf, fage. ten. zoonig, unten wollig und faflnerdend; bie Slattanfítze lanzetfórmig, binfällig; Die Slumenblîtter faft linienformig (Woln. (siebirge. 25lbj. Shay)

Amelanchier,

3 bie Blåtter fágegífnig, enfórmig lanzet. Jlúbbitme.

\section{ailinlids}

a unten befaart; bie Blithentolben fitels

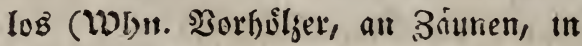
(Sairtelt. Zll); Nay. Gebr. Die Fruedte ein vortreflid)es Dbft, geben Moft, Effig, Sirandrwein; Die Blütben geben teid)s

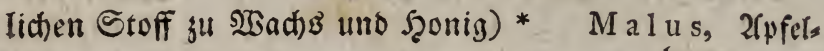
glatt; Die Blüthen fraukfórnig (WWbn. baum.

31b). U. Gebr. wie obcr. DaB frols ifteines ber boirteften, tougt gu allers (ev) feften Gierithichaften, und ftatt (Ebengolis) * : = communis, Viin,

(21um. bende legte Arten babell uns baum. jåblige Abarten, bie in Giarten gezogen werden.)

\section{Q. \\ Quercus, Eidhe.}

1 Die Şlätter lỉnglicht, gefiebert, buchtig, yor, wairtz breiter; bie ફudtiten etwas fpiţig; Die Ecten fumpf; bie Fruichte faft ftiellos

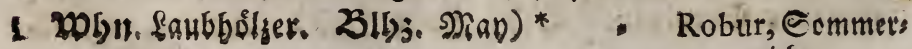
eiche. 
2 Die Bucten fumpf; Die Slattftele furs; bie Fruid)te langgeftielt (WOLn. Waldun.

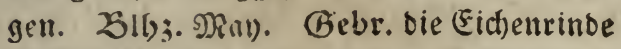

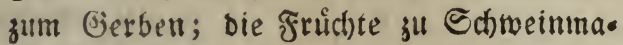
frungen, auch ju Eaffee. Dab Jcoly ift fefre

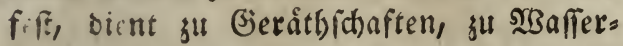
gebåuben, Iro̊gen 4. D. gl. vorzuglich) * fructipendula, Steineidje.

\section{$\mathrm{R}$.}

Ranunculus, Şahnenfußs.

I. Die Blätter gang,

a geftielt,

I berzformig, fumpf; der Etengel ets nas nieberliegeno; die Slume viels blätterig (Don. auf fetten, etwas feuchten \$låtzen. \$15j. Mairy-

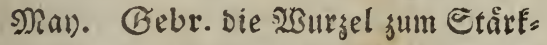
mebl; die Dluthen Den SBienen) * Ficaria, Edjals

2 lanjetformig, etwas gejålut ; der bock.

Stengel auffebend (tohn. felthte

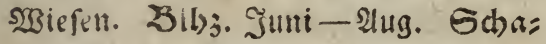
sen. Simbern und Guafen, wenn fie broon freffen, iff er tustlich)*

Flammula, Eleinet.

b fitellos, largetformig; Der Stengel rofb= rig, aufrecht (Won. Wanfergräben. కll;. Juli. Gebr. Den ઝienen) * Lingua, grobet.

II. Sie Blåtter zerfit)nitten,

a bandfermig,

a sipaltig; die Etudfegerfdnitten, glatt; Die ふllåtter am Cirunde ber Slütbes ftield)en linienformig (Whit. Wấls Der)

platanifolius, 》las tamusblatteriget. 
b bie untern geftielt; bie obern fitelo log, tief vielipaltig; Der Stengel einblutthig (WDH. SEerge. 23lbj. Jัเni)

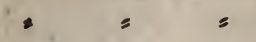

b burchaus 5fitrgerig; bie Finger langets fơrmig, zerichnitten, fågezófnig (Wohr. Gevirge. 2ذil)3. Juni, Juli) .

c spaltig, lappig, geferbt, fommetartig; bie Slattfiele, Der Etengel, sie Shlus thenfticle uno Selche raub; bie Sieldye

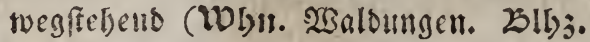
Juni - Saerb(i) *

d 3theilig; Die Ibeile vielfartig; bie obern linienformig; Dev Etengel viels blitthig; bie Shluthenftiele rund; bie Selde wegftebeno (Whn. Sisiefen. 231b). Nab), Juni. Eebr. Den Sies nen) *

acris, firatfer. lanuginofus, twok liget.

aconitifolius, eis fengutulatteriget. babuenfuE.

- Galbtellerformig, 3lappig; die Rappen eingerdnitten; ber Etengel friecheno, bebart (1Dbts. Tháler) : - ferpens, fableident

f vieltheilig; sie Reld)e wegftebend; bie 
3 ber Stengel ranfentreibent; Die Rel. (i)e wegftebend; die Bhitbenftiele ges

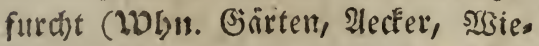
ren. 23l53. Nav, Juni. Gebr. Den Sd)meineri) *

*

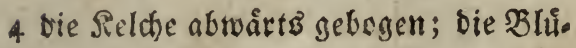
thenftiele ecfig, gefurd)t (WObn. (S)ir.

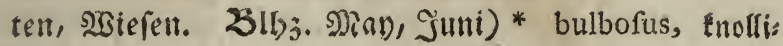

h bie obern Szlatter mế) regts; bie Stúcfe Iinienformin; bie Saas men Dornig (Wolnn. 2lecter. Blbj. Juni) *

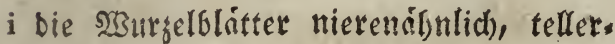
formig, gefpalten; Der Etengel weits

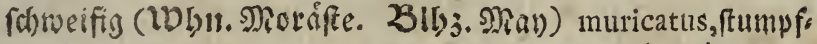

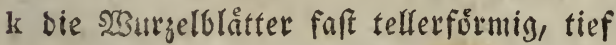
repens, ranten: get.

arvenfis, 2(dêt: babnenfu[. Dormiget.

3paltig; Das einjelme Stengelblatt lanjetformig; Der Stengel einblütbig (Wblit. Ëebirge. 23lbj. Sommer)

1 bie Sצแ alpeftris, $\mathfrak{Z}($ pen. babnenfuk. fdonitten, geferft; bie Stengelblátter ungeffielt, umfaffent, foft 7 fingerig; Die Finger linienfirnitg; Die Caamen feinbaarig (Whln. NSiefen. 33ly3. Mian. Gebr. er hat feitte Edjäre; Eam alio obme Gefabr als 3ugemuife getodht wers Den; allein ber siemner fudte fich feit

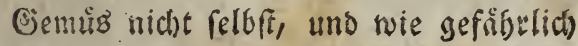
if bierinu Der Jurtijum!)* : auricomus, fíker.

m bie SIJurgelblatter gefitelt, 3theilig; die Theile lappig; ber Stengel einbluttig, foft blattlos; ein funrfingeriges Saß̄. blatt in Der Nitte (TDlyn. (Gebirge)

pyrenaeus, pores nåịน, 
n Die untern Silåter banbformig; bie obern gefingert; bie Frudhtfnipfe långs

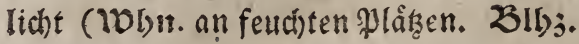
Nan, Juni) * = = . fceleratus, gifti

- Sie untern Şlåtter banräbnlich; bie get. obern nierenformig, tellerionnlich, bands. fơrmig, zertbeilt; Der Erengel fothimmt (Blb3. Juni) = = = diverfifolius, uns

p bie untergetaud)ten $\mathfrak{B l e ̂ t t e r ~ v i e l f a c h ~ b o r s ~ g l e i d b l a ̈ t t e r i g e t . ~}$ ftenformig zertbeilt; bie obern 3-5lap= pig; bie fappen abermal lappis; ber Etengel fatwinmt (Slij) Nian)

peltatus, (chilos blätteriger.

\section{Raphanus, Siettig.}

a Die Schotten glatt, vielgliederig, lang, eins fricherig (Whn. Aecter. 25!h;. Sommer. Gebr. Jutter, Den Sienen) * , Raphaniftrum, bie Edyotten Enotig, swenfơd)erig (WWhn. 2lekertettig. (3irten) *

2(barten bes gemeinen Diettigs, die in unfern Giêrten gejogen werden.

a) Die sisurgel anfehnlich grofi, fdarfo

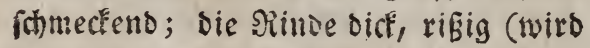
um Jobannis geftupet) $\Rightarrow=$ hiemalis, 2 Sinte:

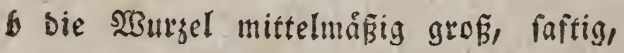
rettig. nid)t gar (d)arf; Die Rinde nidjt risig (mird im Nan geftupft) aeftivus, Somi

c bie 2 Surtzel flein (werden meift in Mifts mertettig. bettern gezogen)

* li̊nglicht),

I fohwars ober tweî . " vernus longiufculus; 2 refthlichts, purpureus, Forellen: rettig. 
G tumb, det Sthwans sum

$$
\text { Refeda, Siefeoce. }
$$

vernus orbicularis, ruttoet 9)?o= natrettig.

a Dic Blátter einfad), lang gezogen, lanjet. âfnlid); an Grunbe zu benben Seiten mit eittem fnorpliditen 3abne (10bn. als Ientbalben an 3 segen. 23lis. Jumi, Suli. Gebr. zum Gelbforben, Den Bienen) * Luteola, 2Bau.

b Die Slatter 3theilig, Die untern gefiedert (Whin. auf Jergelgrüben. BIbj. Som= mer, Serbir)*

3

lutea, gelbe.

Rhamnus, ngsegoorn.

a Die jüfrigen 3roeige an ben (Enben fact)= (id)t; bie Slătter enfôrnig, fägezånnig;

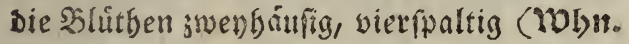

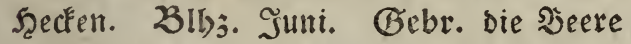
unb \{inbe zum färben; Das faub jum Futter bes Sindviebes; bas gelbe .golz รัt berfdiebenen 2 rbeiten)*

b die 3meige frachellog; bie Blätter gans; catharticus, Sieufoorn. sie Sluethen Bwitter, cintweibig (Whbr. Jecter. 2ib);. Juni. Gebr. Färberen, Sienen) " $=\quad=$ Frangula, Fauts

Rhinanthus, Silapperfiaut.

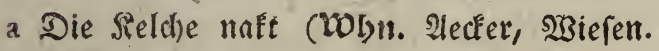

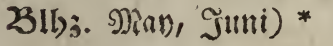

- Crifta galli, ger 23153. Juni, Juli) *

Rhododendron, Illpenbalfan.

a Die Slltme tridterformis,

I bie Blätter glatt, unten von gebringten Druifen rofffórbig (WDin. Bebirge. 2(1)3. Iแแni, Juli) ferrugineum, rofs färbiger.

a bie 
2 Die Slatter mit Sorfiten gefranzt, auf Der Unterfeite mit punftfórmigen Drús ren meitlóufig befegt (WObur. Giebirge. 2lb3. Suli, 2iuguft) = , hirfutum, jottis

b bie Blume rabformig; Die Slátter borftig gefrangt (WDbn, Gebirge. Blbj. Juli) get.

Chamaeciftus, ciffformiget.

Ribes, Sirausbeere.

I. Stadjellos;

a bie Slůtbentrattben bangend,

1 glatt; bie Reld)blitter wegftebeno (Whin. Gartanbeden. 23lb: Mian. Gebr. Die Seeren rob und cingefot. ten zur Epeije) *,

rubrum, Soban.

2 bebaart; bie Sllithen linglidht, mit nisbeeren. puntfformigen Drúien befezt (WWhn. Gartenlieclen. 2b!b;. Nav. Bebr. zum (Enen, Dranbtwein, ben Bienen)* nigrum, (jid)tbees

b bie Slutthentrauben alfrecht ober weg. tert.

ftehent ; bie Slattftiele viel füryer als Die Drenfpaltigen Slatter (Wobn. Gies

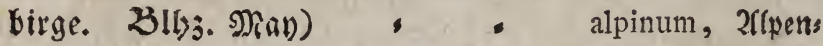

II. Stachelidit; johannisbeeren.

a bie beeten glatt; bie \$luethenftele eit. selin, mit einem eingelnen blithenblatte; bie-Slitter faft tellerformig, lappig, runb geferbt (10bur. Şecten. Z3lbj. May. Orebr. Epeife, Eirig, bie Slü then Den Bienen)*

Uva crifpa, Striat

b Die Trauben nufredt; bie Beeren rauh; fetbeeten. bie Slattfiele gefrangt (WOhn. Barten. becten. 23lb3. Nay. Gebr, wie bie obige 2(rt)*

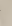

Grofularia, Sirof felberten.

Robinia, 


\section{Robinia, ๔chotenoortt.}

Die Blitben it Tranben; bic Etield)en ein: bluthig; Die solitter ungepant gefiebert; Die Silattanfôtze ftad)lid)t (Mbn. biod)ft felten fieft man ifn all ben b̧âfern ber fandleute gepfonset)

Pfendoacacia, ges meiller.

\section{Rora, siofe.}

I. Die Frudtennoten glatt,

\section{a enförnig,}

I bie felche faft gant; bie Griffel lang, gewunden; bie Slatter glatt; ber Etengel bornig, weitfdyeifig; bie

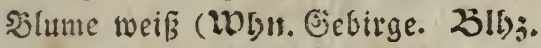
Juli)

2 bie Reldie balb gefiedert, gottig; bie fylveftris, $23 a$ alo, tope. Bheithenftiele glatt ; Der Stengel aufo recht, unb fammit bem Solattfitiele bor: nig, gan' unbebaart (WDGr. Fecien. 25!b). Juni, Juli. Gebr. bie Slata ter sul Rofend unb Rofentwaffer;

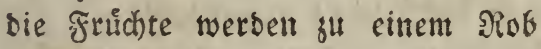
einge(otten) *

canina, Şundós tofe.

3 bie Selche wechleleweis gefiebert, zots tig; bie Slattfiele bornig, zottig (105u. 2luen. 3il);. Juni)*

4 bie Sieldbfitele volffommen gang; bie Emlanteria, 『n: gettoierrofe. Sllitínen = uns Blattfiele unbervegrt; Die Blăttchen enfơmin Iangetånnlich; Der Etengel vicloornig; bie Dornell gernoe, zween ftirfere an ben stab. feln; Lie Şluthenjweige febr zabl:

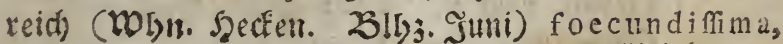
vollohthige. 
b faft futgelformig; bie \$lutthenfitiele glatt; am Grunbe Der SIlattanfåąe Dornen; bie Sllittct)en eyformig, rpişig, unten rammt ben $\mathfrak{S l a t t f t i e l e n ~ f e i n f o t t i g ~ ( W O L n . ~}$ Scecfer. Blb3. Suli) * : cinnamomea,

II. Die Fruthtfnoten glattwerbend, enförnig, Bimmettofe.

a bie Reldbblatter balbgefiebert; bie \$lüs then an ben Enden Der Bweige, faft buinbelfơrmig (WOLn. 23 aldungen. 25lh). Jumi)* , - mofchata, Miıg̀!

b bie SSlutthenftiele fteifborftig; Der Etamm kusto

unbewebrt (Whln. Eebirge. 25ll)j. Suli) , alpina, 2flpemtore:

III. Die Fruthttnoten faft glatt, eyformig; bie Bluttgen = und Blattfitele von Drûfn= borfen raub; ber Etengel gebornet (WWln. Oebirge. Zllyj. Juni). = " collina, Veetgrofe: IV. Die Frudt)tnoten fteifforftig,

A. evfeirmig, pammt ben Slutbenftielen ffeifborftig;

a bie $\mathfrak{b l a t t f i t e l e ~ f t e i f f o r f t i g , ~}$

1 gebornet; bie Seldhe halbgefiebert; bie Slâttchen enfơrmig, fpiçig, fågezånnig, unten feingottig (Wobrt. frecter. 23lb3. May) = gallica, franģos

2 von braunrotben Drifen, mit uns fifiche.

termengten fleinen, gefrimmten rotben Dornen, raub; ber Stamm bben febr bornig, blitbenarm (WDLn. Scecfen. 25l5). Juli) * auftriaca, fleine.

$b$ bie Slattfiele bornig, filzig; Der Stamm naft; bie Slåtter fil zig (Wbir. Saeken. 23153. Juni, Juli.

Gebr. wie oben bie Saunderofe) * villofa, zottige. B. fugels 
B. fugelformig, fanmt ben Blattfitelen fteifborftig; Die Dornen: Etunnt; Die Slatter unten mit rofffárbigen barz̧is gen \$umfen befegt; die Slume gelb

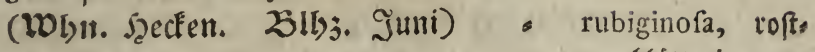

Rosmarinus, Siosmarin. biatterige.

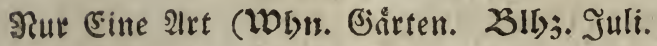

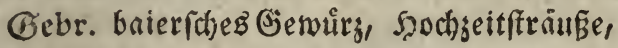
Den sienten)*

\section{Rubus, ふrombeere.}

officinalis, gemeis net.

1. Die Slåtter fưnffingerig sefiebert, uns 3fingerig;

a unten filgig; bie Slattfitele boblfeblig; sie Seeren roth ( Jumi, Juli. Gebr. sut Epeife, zum Effiganfeţen, ben Sieneri) * = Idaeus, Şimbees

b bie \$lattfitele bornig; Die \$eeren fdwar" renftraucf.

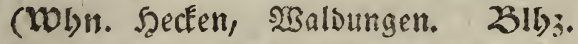
Jumi, Juli. Gebr. Epeife, ben Sies nen. Gdas. Dem holganfluge) * fruticofus, Broms

II. Die Slátter zfingerig, beerenftraudy.

a Ppisig, glatt; bie Ctengel jåntrig, friec cheno (WDIn. NSaldungen. 23lb3. Intli) faxatilis, Etein. b bie Seitenfucfe zmenlappig; Der Stamm beeren. niebergeworfen, Dornig, runb (MOLn.

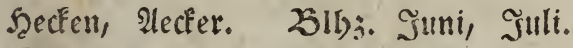
Bebr. Die Seere efbar. Ed)as. als cin (chmer auEzurottenoes UnEraut) * caefius, Raferbes Rumex, SImpfer. tenftraud\%

I. Mit Bmitterblund)en;
A. Die Släter lanjetfirmig,

a vollfommen ganis 


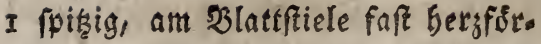
mig, bie fielche gezinnt, mit Dru. fen (WDbn. feudte 2ssiefen. Blbj. Sulli. Gebr. Die sisurgeln zum Eelbfórben) * : $\Rightarrow$ acutus, pipisiger.

2 beråånlich), am Ende fumpf; bie Duirln an ben Epinen ber Stweige

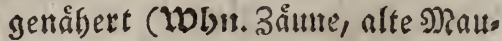
ern. 2lly. Commer) - obtufifolius,

3 fein wellenformig am Ranbe; bie ftumpfer.

Sielchblåtter mit einer grofien Drůie nutf bem Situcfen; bie Slutthen in Duirln (Wobn. wie bie obigen. 2)เร3. 2(ugu(t)

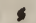

4 fchmal, wellenlos; die Sielche ges zábnt, mit Druifen; bie Duirle Des Stamms uno ber Alefte von einem lónglidbten Sblutbenblatte

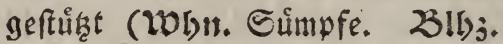
Juli, Aนguft)

b ettwas fraus, feit geferbt, lainglicht, bie Silattfiele oben flach; bie freld)e srúfenlos (Woltr. sisaffergriber. 23lb). Sommer)

c fraug, geftielt, bie Slattfiele benders maritimus, Eees ampfer.

undulatus, bes willter. feits gefurd)t (10bur. feuchte 3lij;. Juni. Gebr. Den Gerbern)* crifpus, frauler.

B. bie Slâtter bergformig, (pondonibnlich (Whn. (ianten. 23ibj. Man, Juni. Gebr. 3แgemน์ Siråuter(uppe) *

II. Mnit getrennten Gejaled)tern;

fcutatus, Sinttens almpfet.

a Die Blátter lănglicht, pfeilfơrmig (Wobrr.

S2siefet. Slly. Jumi. Gebr. in ber Sirues, ben Gerbern, Fe̊rbern, Futters fraut) * 
b bie 3 latter langetåthlich, ponbonforemig (10bn. Recter, an 2 segen. 3lib3. Miay. Gelor. Den Edfafen, (jemis) * Acctofella, Edjaf III. Mit vernengten Gielchled)tern; Die Blát. ter frumpf hersformig, rumglict)t (WDhur. Eebirge. Slbj. Jumi),$\quad . \quad$ alpinus, Misncts: thatarbar.

\section{S.}

Sagina, Mrapterant.

a Die 2hefte nieberliegeno (MLn. 25eiben. 216). Juni)*

b Der Etengel aufrectit, I-3bluthig; sie procumbens, nie: sisurgelklitter nieberliegens, zablreid) um Den Etengel (Blbj. IJan) = " eiecta, aufichteb.

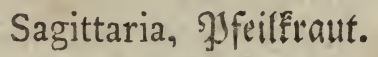

Die Şläter pfeilformig, fpithig (WOIn. $23 a$ f fergráben. 23lbj. Juli) = , fagittifolia, ges

\section{Salix, 2ISeibe.} meines.

I. Die Slatter lanjetfórmig,

A. Rågejåf)nig,

a glatt;

1 grunowoirts fdyôles, gahnlos; die obern gegenuber; bie månnlide Bluthe einmonnig (Wonn. 2(uen. 23lly. Itpril) *

2 chunal; sie erfen jabllos, bie fols Helix, Fofen seibe. genden frigegribnig; bie unterften sesenueberftebeno; bie måmnlid)en Silatter einmonnnig (20 2515;. Wie bie vorige) * = purpurea, purs 3 Lie פlattanfrize ectig, fi̊gesáfnig (Whit. Sofgmerwalo) (id) blitterige.

b ben: 
b Eenderfeits mit einem peibenartigen Szeren überzogen, fpibig, fitmal; bie

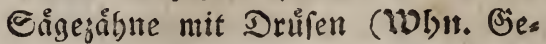
ftade. Zlbs. Ipril. Gebr. Die Saas menwolle zu Şuten; bie Rinbe ben Frarberw) *

a 1 ba, Silbet; weide.

c oben behaart, unten filgig; bie Griges zâfne mit Druifen (Lobu. 2uen. 2315). Fruibling) * incana, graue.

d unten feibenåfulidh, grau; fdomal, ftiel. los; Der Stamm niebergemorfen; bie fefre weitlouftig und feid)ten S: $_{\mathfrak{a}}$ gejähne bedruffet (Woln. 2uen. Blbj. 2pril. Gebr. Den fiorbflecthern) * rosmarinifolia,

e zugefpişt; die Srigeg̊̊nge brưfig; bie rosmarinbláts Slattfitele mit fparfamen Druifen be= terige. fejt; an Den oberften Blattern febr Hleine, fägezánnige blattanfáse

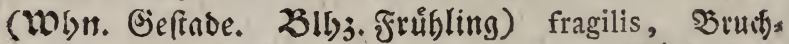

B. volffommen gatts, weide.

a fatmal, oben faft naft, unten bid)t mit reibenåhnlichen, langen, anliegen. Den Scaaren befleibet; Die $23 u r z e l$ friecheno (Mbit. Miorafte) = incubacea, fries

b benderfeits befaort; Der Stamm frie djente $\mathfrak{S B}_{\mathrm{B}} \mathrm{l}$ f ts

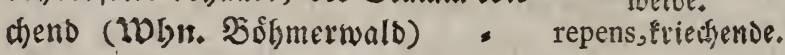

I. Die \$látter an benden Enden langetfó: mig zulaufend, unten faum merflich bes baart; Die Căgezăbne mit Drifien (Moln. 2tuen. 2lbj. Frubling) = " rubens, rothe.

III. Die ミlåtter lanjetfơrmig evruno,

A. glatt,

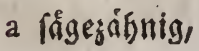


I bie Sågesifine fnorplidyt; bie Blattficle mit groben fitillofen Druifen (DOln. Oeftade. 23l53. Fruibling)

- vitellina, get6e:

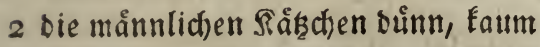
bebanrt, gefårbt, 3moinnig ( $(\mathfrak{O l}$ )r.

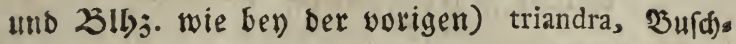

b bie. Slluttben vielmånnig (WDLn. Ge: weide. birge. 23!bj. Frůhling. Gebr. ̧̧ur Watte, Den Bienen) = " polyandra, Lot

B. oben fammetartig, unten faft fillig; beetweide. am Rande nellenformig; bie Sêtąt;en

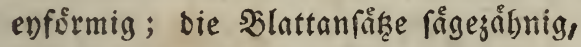

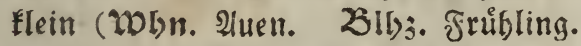
Gebr. Den Gierbern, Den Sienen, Farrs berey) *

IV. Die Slåtter elliptifch, vollfommen ganz, Caprea, serfts socive.

glánzent, oben glatt, unten fein befaart; bie Slattanfinge feblen ( 23lbj. Fruibling) = . , fufca, biaune.

V. Die Slåtter enfoermig,
A. fẩgęafbnig,

a lang gezogen, unten zottig; Die Såge. zoibne frumpf, mit Drúfen; Die jun. gen Triebe und bie Sjlattfitele jottig; Die Blattanfâtąe fảgezånnig, mit Drü. fen, balbherzformig (Woln. Eebirge) cinerea, afdigraue

b feinaberig, oủn, gebáuft; bie Seld). Bergweide. fatupen auggerandet; Der Etamm fraud)artig (Wolnt. Siebirge. 25ibj. Iuni, Iuli)

c glatt, aberig, fpizitg; die saldhe ge: framat (WDyn: Oebirgs)

Arbufcula, (trauct)s attige.

Myrfinites, f(d) warzoornblăttes rige.

B. am 


\section{B. ant Rande gants}

a oben naft, unten filsig; am noufern Enoe zugerundet, mit einer furgen Epifze (Bli)j. 2pril) = = acuminata, fpiks,

b unten feinjottig grall, nek̨formig, ঢlåtterige.

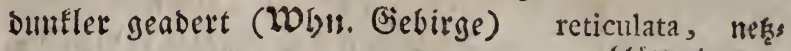

C. autgerantet, ungez̧ånt, glatt (WWli. blätterige. (Jiebirge. 3ll53. Juni) s, "retufa, abges

V. Die Blåtter verfebrt enfïrnig, gang, meis ftumpfte. fiens an benoen Seiten bebaar; furze, (d)ief berjformige SSlattanfâtze (IDLun.

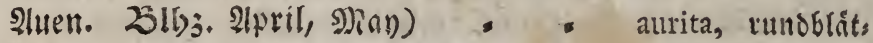
terige.

\section{Salvia, Galbey.}

a Die Släter länglid)t berşôrmig,

I alle geftielt, lappig zerfduitten, fágezấ. nig, untelt beljant, oben glatt; bie Shlutbentriebel entfernt; die Sield)e und Slutbenblatter ftarf bebart (Woltr. SWaldumgen) = " = bavarica, Baiels

2 sie obern umfaffend; bie Duirlen gient. icher.

lidi) naft; Der feelm flebrig (Wolytr. Stsiefen. 2Ibs. Miay, Juni. Gebr. Den (jerbern) * " . =

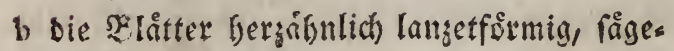
pratenfis, 2̧iefen: jalbey. zånnig, flebrig (Whn. Iluen. 2lb3. 2lug. - Sept.) *

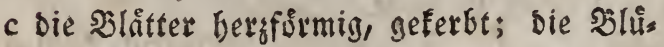
glutinofa, $\mathfrak{k l e b s}$ richtet. then in tingformigen, foft nokten Duirlen

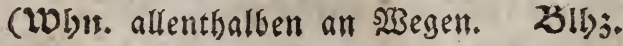
2ug. Sept.)*

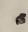

verticillata, quills blůtbtget. 


\section{Sambucus, Şollunber.}

a Saumartig,

I bie Şluthen in einer 5 theiligen Irtige soloe (Woln. S̊åne, Giårten, an Nau. ern. Zills. Sunt. Gebr. Speife.

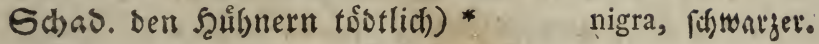

2 Die Trauben zufanmengefę̧t (WOJn. N3albungen. Z(bj. Nał) * - racemofa, Traus benbollunder.

b frautartig; Die Slitbenrifpe 3theilig (20hn. an 2 Báldern. 23ll)3. Juli, Ilug. Gebr. bie Seeren jum fărben)* . = Ebulus, 2(ttid):

\section{Samolus, §unge.}

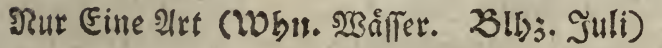

Sanguiforba, నBiefentnopf.

Die 2lebren enformig (Whn. STiejer. 23ll)j.

Juni. Gebr. Den (jerbern) * - officinalis, wilbe

\section{Sanicula, Sanifel.}

Die Srutrgelblétter tellerförmig, 5theilig; bie Theile 3lappig, fâgezátgniz; Die כblůthen alle friellos (Won. Raubbólger. Z3lbj. Nay, Iuni. (Jebr. ven \$ienen)*

europaea, gemeis net.

\section{Saponaria, Geifenfraut.}

Die Seldje rolyenformig; die Slátter en. fớrmig, lanzetăhnlich) (Whrt. SBalle, Etraf

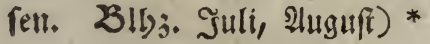

officinalis, gemeis nes.

\section{Savaftana, Savaftane.}

Die Epelgen mit furgen Steifborften; am Sande baarig gefrangt (Hobn. 2hen 231 j M(an) *
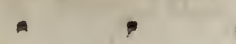

hijta, เauge. 


\section{Saxifraga, Steinbret5.}

1. Die Silétter ganz

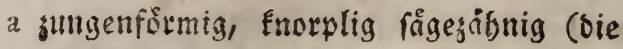

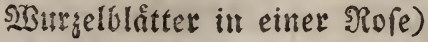

I ant Birunde gefrants; Die Sluments blătter zungenformig, mild hrabmfårs biģ, mit Dunfeln \$utten (Whyt. Gevirge. 2కlly3. Juni, Juli)

maculata, punftit:

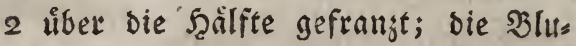
ter. menbläter lanzetformig, fattgelb (WDbur. Oebirge. 23lb). Juli, Mlig.)

3 ungefrangt; bie Sllumenblåtter sut. mutata, selbet genformig, ofne \$unfte (Wobn. Sies birge. 25ilj;. Juli)

Cotyledon, mabele

b linglidft evformig, zurudfgebogen, sben frautartiger. vertieft pumftirt; Der Stengel arnblü thig; Die 23 urgelblatter in einer Rofe (Whur. Bebirge. Bllyj. Juli-Eept.) caefia, mee: c bie Stengelblatter wed)felfeitig, gituet.

I linicufórmig, mit Eleinen weithen

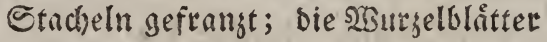
gefauft (Whur. feuchte Felfemiken; Slb;. Illli- Eept.)

a u tum na 1 is,

2 lanjetformig, ghatt, ber Stengel auf J Jetbftfeinbred\%. rectit, mit roftiger sisolle befleidet, armblithig (Mobn. Sumpfwiefer. 21)3. 2ugnif) $=5$

Hirculus, fottiget.

d bie Stengelblätter feilförmig, faftlog;

Die mtern an Sorberende 5 zalnnig; bie น̊brigen 3zăbnig, bie oberften länglicht unb jabnlez (WOLn. Ojebirge) " afcendens, breys e Die Stengelblåtter nievenformig, gežnhn, záfniget. geftielt; Die Slutthen in einer Silpe (WhI1. Gebirge. 25lbj. Juni, Suli) rotundifolia, 
f bie Etengelblitter feblen faft; die $2 B u r$. aelblintter feilformig, lönglic)t, frumpf, von Der Nitte an gegábut; Der Sten. gel ęifig, gerifpet; bie blumenblätter am Girunde mit 2 Sropfen (WOhn. Als pen. Blbj. Juli)

II. Die Blätter lappig;

ftellaris, fternfor: miget.

a Die Etengelblatter nierenformig; Der Etengel iffig; Die Sigurjel fornig (WDIn. - magere Grunbe. Blls3. 2tpril-Juni. (Gebr. Den Sienen) * : =

b bie Stengelblåtter keilfórntig, 3îpaltig, granulata, forni: get. wect)elfeitig, faftig; Der Etengel aufs recilt, âftig (WDlsur. alte Mauern, Fels ren, siecter. 25lb3. Fruibling) *

tridactylites, Şins

c bie Etengelblätter rehr menig; bie uns getnigelf́raut.

tern Silátter 3haltig; bie ŞBurgelblät ter in einer গiofe, tief sfpaltig, bie Etucle linienformig, allseinander fies bend (Wolstt. Gebirge. 23!bj. Juli, 2inguft)

s

d Die 2 sutryelblåtter linienfơrmig langets ăbnlich) uno gaùs, auch feilformig uno 3fpaltig; bie Edbifte vertrodnet, meis ftens einblutbig; bie blumenblätter fchmal lanjetformig (TOlyu. Bebirge.

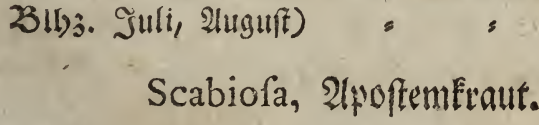

I. Die Sluimchent 4 fpaltig;

a bie am Umfreife groffer;

I bie blätter långlid)t eyformig, fäges zăbnig, unzertbeilt; Die Etengel fteif.

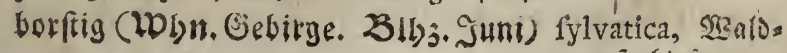
frabioje.

mufcofa, moos: affnlicfer. bildendet. 
2 Sie Slitter gefiebert, jerfditten (20)3r. an Etraffen. 25lb3. JuniGept. Gebr. Den Bienen, $\mathfrak{Y} i e \mathfrak{s}=$ futter) *

arvenfis, 2fefer: icabiofe.

b gleidfformig; ber Stengel ziemlid) ein. fad); Die Slatter enfơrmig, lanjetribus

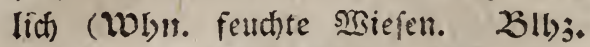
2lug. Cept. Gebr. Fårberey, Den sies nen) *

fuccifa, 2ab6if.

II. Die Şlumcten 5fpaltig; bie am Umfreife gro̊fer ;

a sie sisurgelblơtter exformig, geferbt; Die Otengelblätter gefiedert; bie Şlät. chen linienformig (WO!n. $25 e g e .231 b 3$. Juti) *

$b$ die Slätter boppelt geficbert, zerfanits columbaria, Taus benicabiofe.

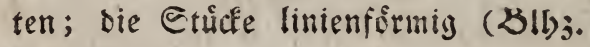
Alug. Eept.)

\section{Scandix, Fiurbel.}

ochroleuca, gerbs (iid)te.

I Die Eamen glatt, geftrcift; bie Bilntto chen lanzetformig, gefiedert; bie oberfen gefiebert zeriahnitten; bie lejten Blättchen lanjefformig, eingefdinitten; Die Slatts

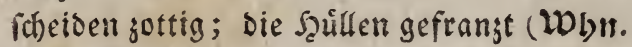
Bofhmerwalo)

- odorata, mofiries

2 bie Saamen enrund pfriemenformig; bie d)ender. Sa)irme fitellos, an Den Seifen (Winn. Gärten. Gebr. it ber fiecche) * - Cerefolium, gee

3 Die Gaamen evforrmig, ffeifborftig; Die meinet. Slumen Durchaus gleich; Der Etengel glatt (WDbu. an Báunen)

Anthrifcus, $\Re$ let: tentorbel. 


\section{Sherardia, Sherarbie.}

Die Slatter burdfaus in Duirlen; bie Slu. then an Det Spitze (WOlyn. auf Hecfern.

\section{2jlbj. Sommer) ${ }^{*}$
Scheuchzeria, Sheudsterie.}

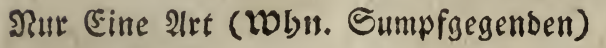

\section{Schoenus, Sinopfgras.}

arvenfis, $\mathfrak{A}$ (efet: idjeratdie.

I. Dek f̧alm naft,

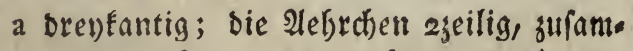
mengedruifft; bie f̧ůlle einblătterig

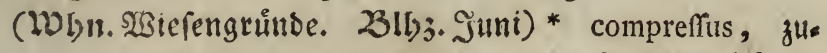

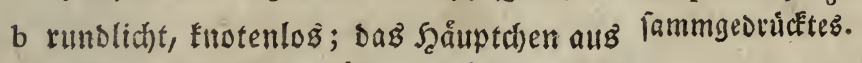
2 Hegren; Das groffere Sâlglein bes Şåntchens mit einer langen Spiţe (Wobn. SiBiefen)

II. Der Scalnt an eitrer Geite flad), ant ber paluftris, Eumpf: idseuiffertie.

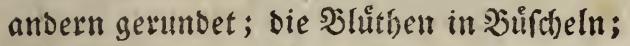
Die Ganmen mit einer borftentrone am Grunoe ungeben; Die blätter borftenfor. mig (I0Lr. naffe 2 siejen. Zlbj. Jull, siinsulft)

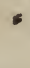

\section{Scilla, NReerswiebel.}

I Der Ėchaft zwenblattetig, armbluthis;

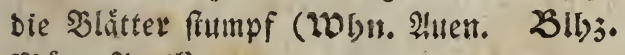
Miár, Mpril) = = " Difolia, Etem,

2 ber Echaft edfig; bie Sllitben iparjam, nigricans, icturitz: (iit)teร.. wed)felfeitig, etwas huberhangend; Die Etiele aufred)t, faft angebrilutt; bie Bláts ter begenformig, mebrere (IDbu. Orag:

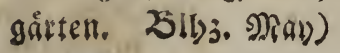

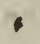

amoena, borta: genolumige. 


\section{Scirpus, Sinjengras.}

I. Det halm nât,

2 runo, (bie Alere ober Albren an Der Spitie)

I borftenfơrnig; bie Aebre armblithig, zwenflappig; die Saamen maft (WOnn. 2tuet. 23153. 2litg. Sept.)*

2 bie $\mathfrak{A}$ ebre enformig, Eegelafulich acicularis, 2ueflo bingengras.

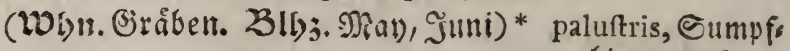

3 mefrere enformige geficlte Aebren binzengrab.

(WDir. Grioben. Blbj. Juli)* lacuftris, Eeebins

b geftreift; sie 2lefre an ber Epitse von zengrar.

Der fange der einfchließenden Blithen. bläter (rohn. feudite Saiefen. Zlhj. Sumi)

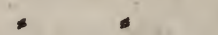

c borftenformig; bie Aefrell fehr fparfam cac fp it ofus, Mioorbinzengraร. alts Den Seitell, georiongt, friellos

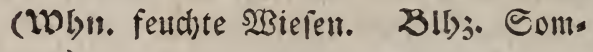
mer) = " s

d 3reitig, futhig; bie Sitpe nus ber Srite, artiges. febr furzftielig; Die Ilebren gebăuft (WDln. Oirioben)

II. Der Scalm 3tantig;

mucronatus, freif, ipiह̨igez.

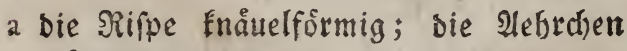
lainglid)t eyformig; bie blutthenichup= pen 3sibnig; Der Mittelgahn pfrtemen= formig (WoLn. Mlfer) $=\quad$ maritimus, $\mathfrak{H}$ fers

$\mathrm{b}$ bie Dolbe vielfad) zulammengefest; bie binzengraz. Hebrathen enformig; bie Slátter flad (Whin. feuchte \$läz̧e. 25lbj. May, Juni)* binzengtns. 
Scleranthus, sinauel.

a Die Frudtfeldie offen (Wobr. Aecfer. 3il). Eommer)* " . annurs, Eommer:

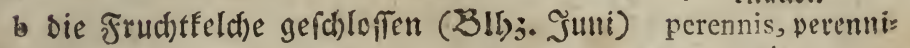
ienoer.

\section{Scorzonera, SForjonere.}

x Die Bläter lanzetfơrmig, mervig, flach, am Stengel faft gar feime;

a breit, ber Etengel glatt, einblutthig (Whit. STiefengründe. 23lbj. Mal). Gebr. ben Bienen; Schweine fuchen bie Sisurgeln gicrigft) * " humilis, niebrige.

b ber Stengel gan einfach, an Errumbe

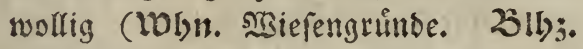
Maท, Jแแni)

2 bie Slåtter linienfơrmig,

a pfriemenfórmig; ber Stengel långer alB Die Slatter, faft' áftig, am Girunbe zots tig; Die Reldffhuppen giemlid) ftumtpf

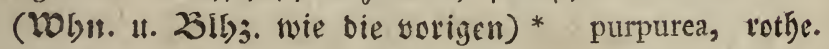

b langzinnig; Der Etengel aufredt); sie Selchfchuppen zugefpitzt; bie Spizen wegltebeno (I0III. 11. 23Ibj. wie bie vorigen)

- $=$
phularia, Ṡraummoury.

\section{Scrophula}

a Der Etengel 4 fantig,

I bie gianten gefligelt (WOhn. Giåben. 3lyj. Sommer) *

2 bie Ranten ungefligelt, ffeifforftig (Wh). laciniata, zetrifo Fenbtâtterige.

la nat a, molfige. 
b bie $\mathfrak{B l i t f e n f t i e l e ~ e i n j e l n ~ i n ~ b e n ~} \mathfrak{B l a t t}$ winkeln, mebrblütbig; die Slåtter ges genúber

8

vernalis, gelbe.

\section{Scuttelaria, Shildfraut.}

Die B̊látter bersfơrmig, lanzetåfnlid), gekerbt;

bie Sluthen altz ben Ad) feln (Wols. 2luen.

$\boldsymbol{D l b}_{3}$ (Sonmer) * = . galericulata, ges

Secale, sioggen.

Die åupern Spelgenflappen ftachlid)t ges frangt *

\section{s. \\ - Sedum, Scoum.}

cereale, gemeinet.

a Die Slåtter enformig, flach, fågegannig;

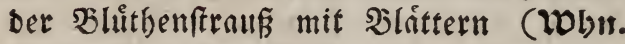
trocktue Girunbe, Slbj. Juli. Gebr. ben Sieneti) * $=$

Telephium, fette Jृenne.

b bie Şläter faft eyfơrmig, hiber bem Grunbe angewadifen,

I etwas aufiect)t, rưcfwårtz gewoelbt; bie Trugbolbe 3tibeilig (WDLn. fteinige Girůn be. 23!h) Juni, Juli)* = acre, fichares

2 all ben juingern 3tweigen 5 - 6 seillig (WOb)t.

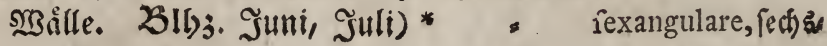

c bie Slítter rundlid)t, effiges.

I långlicht, frumpf, wegftehend; bie Trugs bolde affig (WObn. alte Mautern. Z3ll)j. Sulli)*

-

2 medjlelfeitig, entfernt; ber Stengel

album, Jiauels ampfer.

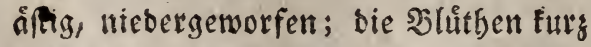
geffielt, entfernt ( $21 b_{j}$. Jutni, Juli) procumbens, nics 
d bie Bläter fainbelformig, etwas niebers gebricti; Die untern 4fad); Die Srugbolde 4theilig; Die Slumenblatter meiñ, mit ros ther Mittelribue (WDht. Jelfen. $23 ! 53$. Sulli)

e Die Slatter oben etmas flach gebruidt, feinbanrig, flebrig, ber Etengel alifredt, fammt ben siththenfielen feinbaarig, fleb. rig (whr. feudte Cegenden. Zlbj. Eommer) , , villofum, fottiges.

$f$ bie Slatter pfriemenformig, it 5 פieithen ftebent, gebrångt, unten los; die Slithen in unåd)ten Dolden (WDhn. feinige Grun. Silk. Sommer) ${ }^{*}=$
Selinum, Silge.

rubens, tother.

$\checkmark$. rupeftre,
feotum.

I Die Slitter 3 mal gefiedert; bie erften Slåttchen rechtminflid)t ftebent; bie lezten einfady, langetformig, boer sefiedert zer. (A) nitten; bie Scillen alle abwarts gerid; tet; Der Etengel edfig (TObn. 23ólber)

- bie Dlätter vielfach gefiebert; Die Ieģtent fylveftre, Dlies nid). Dlâtcdjen idumal lanjetformig; bie Girif: fel ber frucht abwairtz gebogen; ber Etengel gefurdit, fipisectig . :

3 bie જlatter boppelt gefievert; bie Slätt. carvifolia, fủm: metólitterige. chen gefiebert zeridnitten; bie Etudfe linien. formig langetfórmig; Die oberften Slâtter einfach weitlånftig gefiedert; bie blåtts (hen cinfad), lang)

\section{Sempervivum, Şausmurg.}

Clabraci, Cbab. re's Eilge.

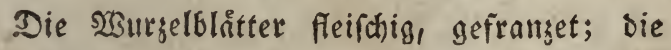
Etengelblatter bautartig; die $23 u r g e l i p r o f$. ren wesftebend

- teetorum gemeine. 


\section{Senecio, গ̧alogreis.}

I. Die $\mathfrak{B l i t h e n ~ f t r a l l o s ; ~ b i e ~} \mathfrak{b l a t t e r ~ g e f i e s ~}$ bett buchtig, umfaffent; bie blutten gers freut (WDLn. Aleffer. 25lb). 2tpril bis Dftob.) *

II. Die Slüthen geftralt;

vulgaris, gemein net.

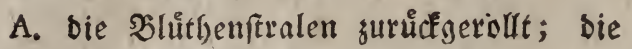
Slătter gefiebert zerf́chnitten;

a flebrig; bie Stúcfe edfig (WDbn. Alefer. ¿lb). Juli) * , vifcofus, tlebria

6 bie Stưfe grobzáfnig; bie Reldie get. glatt; Der Stengel ftraufiantidid (Wohn. Bebaue. 23lb3. Juli, 2ug.) fylvaticus, T3aldi

B. bie Stralblumdhen wegftehend, balogieiz,

a bie $\mathfrak{b l a t t e r ~ g e f i e b e r t , ~}$

a jerfonitten;

I etwas wollig; bie Stucfe liniens fe̊rmig, gez̧ignt; Die Blüther faft buichelforrmig; sie obern von bell untern ůberwadfen chryfanthemoi-

2 behaart; bie Etuiffe langetfor: des, goloblumens mig, gezaifnt; bie Aefte Der Mittelribbe berworftehent; bie Sluttben ftraukfoormig (:Dbn. Sỉniloer) * " alpeftris, $\mathfrak{F e r g}$ jafobstlume.

3 bie Stuicke linienfermig, glatt,
rpisig; bie Slüthen bủichel.

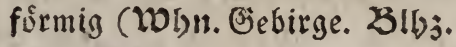
2tıg. Eept.) . = abrotanifolius,

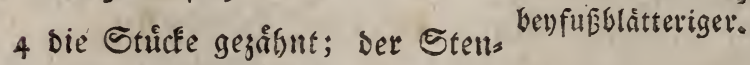
gel aufred)t (WDbr. SOSiefen.

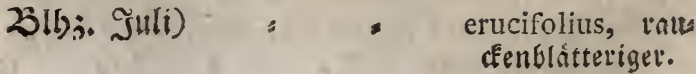

$\mathrm{b}$ levers 
b lenerformig; bie Etricke abermal in flemere jerftuctit; Der Stengel aufrecht (Wonn. 25iefen. Blhj. Sommer) = , Tacobaea, Gnfobs,

$b$ bie Slåtter langetformig, Evaut.

a geftielt,

I unter wollig; Der Stengel ein fach); bie Slluthen foft in Dols Den; bie গ̧luthenftiele ungleid' (Wonn. SWálder. 25lly. Som= mer)

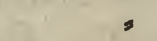

2 benderfeits glatt, fägegáfnig; Die Shluthen in einem Strautie (Wobn. an Bebirgen. 23lbs. Sแแni, Juli) $=$

b fticllog,

$x$ fharf fingesainnig, tmten filsig; Die Stralblutt)dien 16 (WOhn. Mori̊fte. Z3!bj. Jull, Nug.) paludofus, Mos

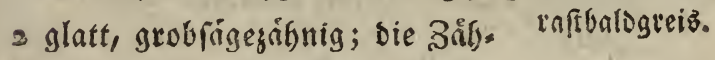
ne mit einer Enorpligen Epişe, gejånt; Der Stengel vieledtig (20)11. 24uen. Z3!b3. 24uguft, Sept.) " " Doria, Doria,

c bie Şlâtter bersfơrnig, grob fäges zålunig, geftielt; Die Slattftiele am Cirunbe nit fleinen Slattobren ( 10 b)

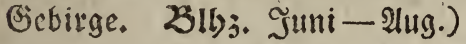

s bie Şurgelolítter enformig, geftielt, nemorenfis, Şain: balogreis.

faracenicus, Şcio: nifchroundétaut. alpintus, 2flperts balogieis.

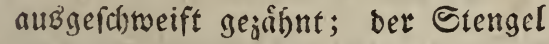
einfad), einblütbig, fil lig ; bie Eten= gelblätter lanzetformig, fiellob̉, făges zơfnig, unten filsig (Woln. Gebirge)

Doronicum, gembioulzábnlis d)er. 


\section{Serapias, Stendeliwurs.}

Mit fafrigen 23 urseln.

2 Die Slitter begenformig,

I ftiellos; bie Slưthen hangend; bie sip. pe ftumpf, am Ranbe mellenformig, fo lang als bie Sielchblätter (Bll);. Suli)

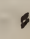

longifolia, lang: bláttcrige.

2 bie Sllitben aufrecht; bie Sippen langets âbnlich), mit rollenfórmigen finten (30hn. Berge. Silt). Sommer) rubra, totbe.

b bie Slâtter enfơrmig langetånnlid),

I umfaffend; bie Bllthen hangeno; bie Sippe lanjeti̊nnlich ( WObn. Serge. 23llj. Juli. Edjadet ben \$ferben) latifolia, falide

2 Die Bhutbent allfred)t; Die Pippe ftumpf,

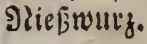
fürser als bie sield)blatter, mit $3 \mathrm{er}=$

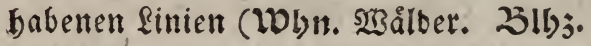
Juni)

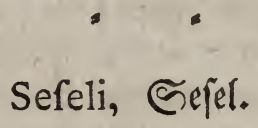

Die Slattfiele am Stengel icheibeformig, aufgetrieben; bie Slåtter mebrmal gefie. Dert; Die erften Slättchen sepaart, Die Iejten linienfórmig (WWhn. magere Gruinde. 2014). 2419.) * annum, einjaiblis
get.

\section{Sideritis, (J)liebfraut.}

Sirautartig; Die Etengel zottig; bie Sieldie gro̊f̧er als bie Slumen, ftad)lidht, nervig; bie Sberlippe 3ipaltig; Die Duiblen blus thenblatterlof (Bllyj. Sommer) *

montana, Vectg: gliedéraut. 


\section{Silene, Gilene.}

a Der Stengel gabelformig,

I unten gans einfach; bie Slatter linien. formig, umfaffend; bie 3wifhentrume Des Etengels in ber Mitte flebrig

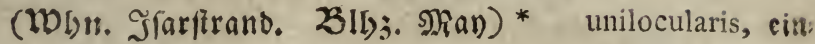

2 bie હiegenblåtter linienformig, wegftes fádjerige. bend; Die Slutthen gefticlt; bie \$lut menblatter 4lappig (Whls. Siebirge. blly. Commer)

. .

3 bie Sieldje roedig, gefárbt, flebrig, mit medrelsmeife iffigen 2loern

b bie \$latte Der \$lumendlitter faft atheilig; bie Tbeile linienformig; Die Frued)te all. recht, furzfitelig; Die Reldhe sfitreifig; Die Slåtter enfórmig lanzetáfnlicl)

c bie Sllumenblätter 2fpaltig; bie $\mathfrak{B l u t h}$ en einfeitig, ůberfangent; bie פiipe abroirts

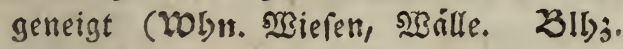
Juni) *

d bie Slunenblatter auşgeranbet; bie Relche etras malsenfórmig; Die SJlätter linien.

fơrnin lanjetännlic) (Wobn. Giebirge. 25lb3. Eommer)

e bie Slutthen in frraufformingen Sindeln;

quadrifida, 4laps pige.

noctiflora, naichts listie.

ceraftoides, horn: Etrutnetige.

nutans, ůGerbans gende.

bie obern \$lätter berfformig, glatt (B!l)j. Iuli)

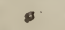

$f$ ber Etengel fitraudartig, nieberliegend;

Armeria, nelfen: artige.

rupeftris, Gerien. filene. bie aufgerichteten 2iefte einbluthig; bie Slutte geftielt (DObn. Biebirge. Zlí)j. Juni, Juli)

acaulis, 3roergs filene. 


\section{Sinapis, Senf.}

Die Chotte effig, won ben Saamen alfs: getrieben, viel lánger als inr Sánabel (Woln. Hedfer. Bllj;. Eammer) *

\section{Sifon, Sifon.}

arvenfis, Wffets ienf.

Die Slätter einfach gefiebert; bie Slattchen breitlitht, fägezånnig eingef(c)nitten; ber Etengel fried)t (Wolsu. feudte Grinde. 23!bj. Sommer) ${ }^{*} \cdot$,
Sifymbrium, Jiaucfe.

inundatum, $\mathfrak{B a}=$ fertifon.

2 Die Edjotten herabhangeno; bie Slatter gefiecert;

I Die Slattd)et enfórmig, lappig (WDLn. șaffergraben. 2bllj. Juni, Juli. , Gebr. Galat)* * = Nafturtium,

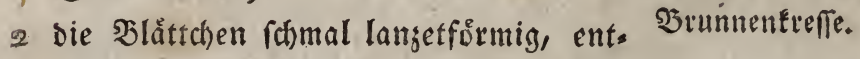
fornt fingezähnig; Das ungleid)e gro̊ker

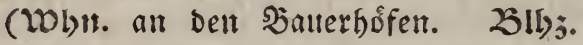
ذuli, 2lıguft. Gebr. ben ふienen) fylveftre, milbe.

6 bie Sdjotten wegftelend;

1 Die Slumen fleiner als bie Relche; bie SBlätter gefiedert jeridnitten (Woln. an Srriben. Slbj. Sommer. Oebr. Den ફ̧ichen * " " : 2 bie blumen groffer als bie Relche; bie Slátter einfach, lanjetfórmig, grob päge. góbnig (WWhn. Gräben. Zlbj. Eom.

mer) *

e ber Stengel åfitg, armblåtterig; bie Slåts ter leverfórmig; bie Stucfe red)twinflid)t wegfteheno, fteifborftig; die Steifborfter

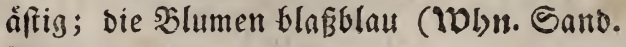
23lbj. Sommer) * paluftre, Moot. grunostaucke.

aquaticum, $\mathfrak{T} a$ a iarnuife. 
d ber Stengel unb bie Slattfiele borftig; bie Slatter bolzfägejatgnig, borftig, fpițig (robn. Bo̊nmerwalb)

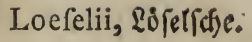

e bie Slumen fleiner als bie Relche; die Slátter vielfad) gefiedert; bie lesten $\mathfrak{B l a ̊ t t = ~}$ d)en linienfismig (Wobr. Felowege. Sll)j. Xiay - Juli) *

Sophia, Sophien fraut.

Sium, Merk.

2 Die Slateter einfacti gefiedert; bie Slatts d)en länglicht lanjetformig; Die Dolden au ben (Enden; bie Umichligge lanjetfór. mig (WVhn. Gråben. 25!b3. Sommer)* lancifolium, lams

b bie જlatter gefiedert; an ben untern bie zenblátteriger. Blättchen geferbt, alt Den obern einges

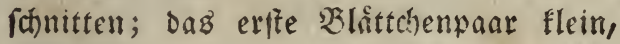
enternt; Die Dolben Den Blåttern gegen.

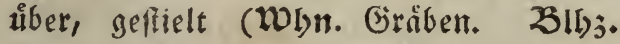
(ommer) * " " " Perula, शुafers

- bie Slåttchen lang, bandförmig, herablau, fend (MObn. Şiefen. 25!bj. Juli)

Falcaria, Eidjets Solanum, Naditfhatten. fraut. mette. 
2. bie blåtter enfớrmig Iangetånnlid), eckig; bie Silumentrauben riberbangeno; bie Slume meiñ; bie beere ichwar; (10bu. Giebege. Zilis. Sommer)

nigrum, idfwats fer.

\section{Soldanella, Drattelblumte.}

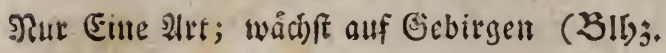

Suni)

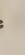

-

Solidago, Sioloruthe. alpina, 2frenorats telolume.

Der Stengel ectig; bie Blitbentrauben aufs red)t, rifpenformig (Wobit. magere Oirün De. 2\$lb3. Juli-Sept) $=$
Sonchus, Eondenfraut.

a Die Solutbenftiele uno Seldye fteifforftig;

I Die Sorffen mit fugelförnigen Drufen;

Die Slatter holgfiggejånnig, ant Cirumbe ber 23iefen. Zibj. Gommer. Gebr. Den Bienen) * . " .

2 bie Sluthenfticle an Enve ber Zweige Virgaurea, golses niอ 2 Bund rinut. genåbert; Die Şlåtter grob holjầges zålunig, am (jumnde pfeiläbulich) ausges fonnitten; Der Situdenmerven ber Silits ter aud freifborftig (Whin. Llfer. 23lbs. Sommer) *

b bie Sllithenftiele filyig, bie Sieldhe glatt; arvenfis, feldsa: jentobl.

\section{sie Silâtter gejåănt,}

I weid), gang, oder gefiesert serf(h)itten; Die oberiten gan umfaffeno ( 20 bn. SMiefen, Gartengrinde. S15j. Som. mer. Gebr. geH $^{*}$ * : = oleracells, semeis

paluftris, Soutm: jondjentraut. 


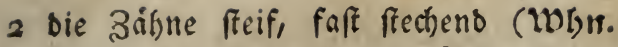

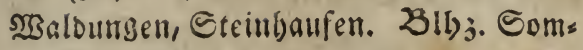
mer) *
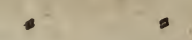

,

afper, Cnubiffet.

c Die Slutthenftiele fhuppig; Die Sllutthen traubenformig; die §latter gefiebert jers (d)nitten; bas ämEerfte Etuck zeckig, röges zófnig, refr grö́ (WObn. SBofmermalo.

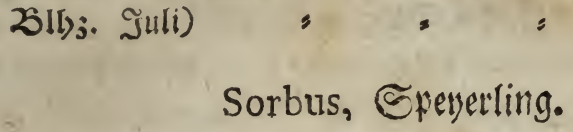

a Die gefiederten Slåtter bender feitz glatt (Wobn. Norbo̊ljer. 23!bj. Nav. Gebr. zum $\mathfrak{2 0 g e l f a n g , ~ F r a n t r o e i n , ~ E i b e r ; ~ D i e ~}$ jungen auggerwachienen 3weige jur (jer. beren; Das Scoly iff bart und jabe) * aucuparia, Bogels

- bie gefieberten Slätter unten filjig ( 21 l)j. May) *

'bertunum.

alpinus, Glauet Şa|enteghl.

domeftica, Epiets lingbaum.

Sparganium, Jgelร̈Enofpe.

a bie Slátter aufrecht, 3fantig (Wolyr. $23 a$ f. rergrâben. Slbj. Commer. Gebr. Den (jerbern) *

$=$.

e re ctum, aufs rect)te.

b bie Blâter auf benden Eeiten flach (20.5n. Gråben. Żlbj. Eommer) *

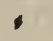

Spartium, ฏfriemenfraut. natans, fifswims mente.

Die Slatter 3fingerig uns einfact); bie Slättd)en evformig; Der Stengel ecfig (DDhn. trocine Sirůnde. 3ll)j. Eommer. Gebr. Den Gierbern, zu beren, Dell Bie. nett; Die Dlüthenfnoipen mit Eals ein. gemad)t ftatt ber Sappern; bie Slumen Den Fonkbern; Die Eaamen ftatt bes siafo fees; Der ganje (teaud) sur \$einmand) * Scoparium, gemeis ne?. 


\section{Spergula, Gpart.}

a Die Blåtter in Duirlen; bie Saamen lin. fenformig,

I randlos (WDbn. WBiefen, Hedfer. Zlly; Friffling und Sommer. Gebr. gutes Futterfraut) * ${ }^{*}=$, arvenfis, willer.

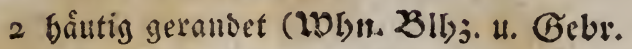
wie bev ber vorigen $2(\mathfrak{t})$

b sie Slatter gegemiber,

pentandra, รuts teripart.

I aufwairts allmäblig fưrser, pfriemenfờr. nitg, glatt; Der Etengel einfad) (WObn. am 23 urrmpe. 31 b. Sammer) nodofa, Enotiget.

2 unverfiust, fefre fithal, an ber Epitze mit eitsm feinen Stad)el, am Sirunde vertrodinet, umfaffend; die ßlumens blåtter fuirjer als ber Sield) (WOLnt. (biebirge. ¿lbj. Sommer) =

\section{Sphaeria, Siugelpils.}

2. Durdjaus roth, fitiellog, baltefugelformig ( Whin. todte 3weige Der Sorume)

purpurea, rothet.

b rotbbraun, $\mathfrak{x} u$ nglid) balbfugelformig (Whyr. Siseibenjweige) *

c in ser Jugend erbaben, tieffa)twars, mit faginoides, rngis nenblătteriget. gelber Rinde; in Alter rindenlos, fugs licht, burdjoobrt; imwendig idwars (Wobn. Baumrinden)

d gebáuft,

mucofa, firimlids:

ter.

I tieffowary, fugelförmig, tunsliclit; ins wendig fdhwars (WDbn. modernde Jals. (ftecte)

- rugofa, imnjlifis

lycoperdioides, fraubpifzartiget: ter.

2 (c) wars 
2 fonwar; balbfugelfơrmig, inmenbig weiß (20)n. jwifhen ben Ed)uiffeldben ber faltempiljühnlid)en Fled)te) - hemifphaerica,

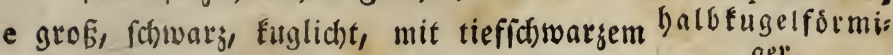
Etallbe bebecft (WDyn. unten am Stamme ber scafelfauden)

\section{Sphagnum, รorfmoob.}

a Die 2 efte abruátz bangend,

I waljenfơrmig; die Blätter faft langet. formig (WDbn. Eümpfe. Bebr. Diefe Slrt Gilbet vorzuglich Den Torf) - cymbifolium, ge: 2 felbr bun, haarformig zugefpitzt; die

maxima, grớctet.

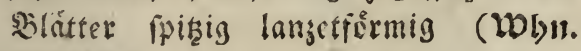
Wioräfte)

b Die Etåmme georaingt, oben riftig; bie meines.

Alte aufrecht; bie ઝlatter lauzetformig, nervenlog, an ben Seiten eingerollt (WDbn. M(oriffe)

\section{Spinacia, Cpinat.} get. 
c bie Blätter einfach) gefiebert (bie Şlithen bilden Seugoolden)

I bie Blättchen evformig lanjetñfnlich; făgezábnig; Der Stengel frautartig (20hn. SBiefen. Z1hs. Junt. Gebr. Den Lobgerbern, Den bienen)*

2 bas ungleidhe Sláttchen grover, lappig

Filipendula, Silis pertid.

(20hn. Sisiefen. 23lbj. Juni, Juli. Gebr. Den Robgerbern, Dell Bienen, Den Fárbern

\section{Splachnum, Edjirmmoos.}

Ulmaria, SBiefene geibbart.

a Der Raprelunte:faç verfebrt Kegelformig; bie Slátter mit ciner Nittelribbe, lamzets forrmig, lang jugefist = =

mnioides, mnium

b Der Rapfelunterfats enformig; Die Rapfel artiges. faft walsenformig; ber Dectel gewoilbt; die Siapfelftiele gewumben; die Slatter enformig, weid)fachlid)t jugefpizt = longifetum, langs

\section{Spongia, Schwamm.} frieliges.

(Birůn, mit filfartig in einntiber verworrenen Fáden, nach) bem Srocfnen grünlid)t grall,

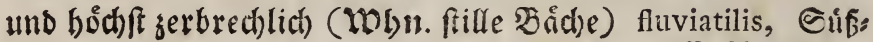

Stachys, Anoortr. wallerfotwamm.

a Die Duirlen in unterbrod)enen 2 ebren,

I blattlos; Die blitter berforentig, ge.

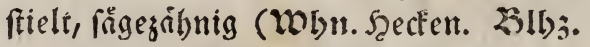
Sommer. Gebr. grůne F̊̊rbe; Der Stengel banfartig)

2 Die oberften gennibert; Der Mittellap= fylvatica, 2 sald, pen ber Unterlippe tief auggerandet; bie Slåtter elliptifh langetformig, faum ges

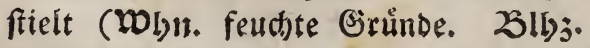
Sommer)

- , paluftris, Eumpf andorn. 
b bie Durirlen vielblůtbig,

I ferbe bebaart, gruinlidnt; bie Unterlippe flach); die Epitąen ber Eágejăbne an

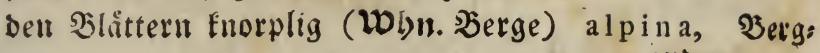

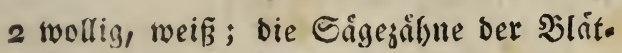
antorn.

ter gerundet, fuorpellog (Whlu. Auen.

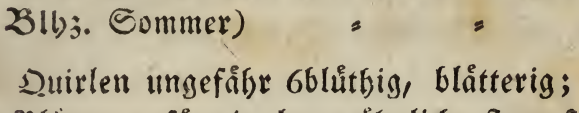
germanica, wobt: riedjender.

c bie Duirlen ungefåbr 6blůthig, blåtterig;
Die Slâtter enfớrnig langetñhnlid), ftumpf fägejăhnig; die oberften ungeftielt; bie Sieldi)e mit Etadtelipitisen; Die Stengel

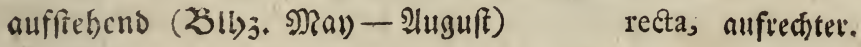

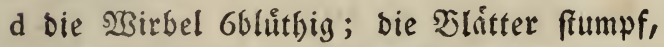
jiemlid) unft; bie Sllumen von ber lănge Des Seld)es; der Nittellappen ber Unter. lippe unausgerandet (LDbur. Aecfer)

arvenfis, Seloan: Dotn.

\section{Staphylea, \impernuß̧̧aum.}

Die Slatter gefiedert (105n. 2tuen. 25lh). Suni. Gebr. bie Saamen geben reidblid) Del)

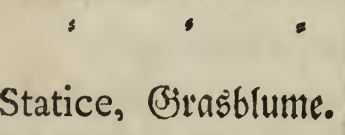

Der (d)aft einfad); bie $\mathfrak{b l u t b e n ~ i n ~ e i n e m ~}$ Sinaule an der Epitze bez Ed)aftes (WDbur. feudte 2 siefen. Zlllj. Ituguft) Armeria, Mieet:
grasnnléte. Stellaria, 2leiriø.

pinnata, gemeis ner.

\section{Statice, Girasblume.}


b bie SBlåtter lănglicht eyfơrmig, fpis̨ig; vie Stengel nieberliegent, faft gablid)t; bie כlutthenftiele einfach uno sblutthig; bie Blumenblátter tief getbeilt, fủrser als ber feelch) (robn. Sandufer. 25ly;. Som= mer)
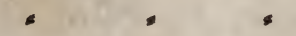

fontana, Quelle

c Die Slátter lang lansetfórmig, fricllog, meitid). gegemiber (bie Slumenblatter zmens. theilig)

I am Sande fein feifborfig; ber Sten. gel an belt \& Seanten freifborfitig (WOLn. शuen. 3!5j. Mav, Juni. Gebr. Den 25ienen $25 a d j 8$ )

2 nicht fteifborftig, am Girunbe geframst

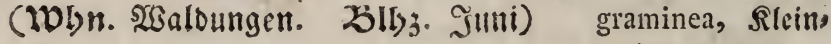
Stellera, Gteller. blumengras.

Die Slátter linienforrmig, wedbrelfeitig; bie

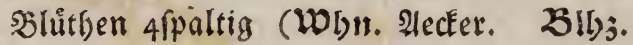
Sept.)

\section{- $=$ " \\ Stemonitis, Siolbenpily.}

pafferina, झante. tine.

a Geftrielt (Die Engaut vergänglich)

I gebåuft, braunroth; ser fruchttolben enformig (Woin. Gólgerne $\mathfrak{Y 3 a f f e r l e i t u m s}$

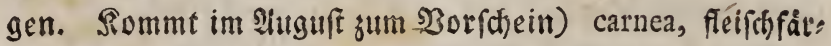

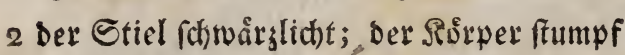
Eiger. -

fegelformig, Dunfelroth; nach verftaub. ten Gaamen flach), Durdirdheinig (Wolsn. auf faulem f̧olge. (⿺廴) b fiellog, zungřfnlidfer.

I gebauft, eyfơrmig, weiñ; bie Eaamen fijwars (Woln. Szafelftaubenrinden.

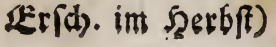

alba, Ueífel: 
2 ber Frudtefolben cufermig, veildentoth (LObn. faules $50 l_{3}$ )

lilacea, filnéfáis

Stipa, ઝfriemengrab.

Die Girannen fefre lang, feberånnlich) (Woyn.

Steingrinde. 2lly. Man)

pennata, gefieber:

teve.

\section{Struthiopteris, EpiEant.}

Die unfrud)tbaren Blätter gefiedert, in lange, fichelformige, aneinanber fiebente Stuicte zertfeilt; bie Fruchtblatter grüser, metts liuftiger gefiedert; die Stúcfe fómåler (Whan. Gebirge)

- Spicant,gemeinet.

Swertia, Sdutrtic.

Die Blume 5fpaltig; Die Nisurglblitter eys

fớrmig (WDin. Sebirge) . - perennis, aus: Dallernoe.

Symphytum, $\mathfrak{E e i n w e l l e . ~}$

Die Slâtter evfơrmig, Inngetåthnlid),

I burd)aus med)iclietitig, berablaufend; bie rsurzel armformig (WDbn. SWiefen.

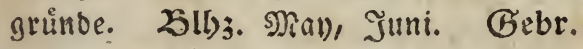
Rohgarre, Fårberel), autch wirb aub ben STluzjela der Siaméelbare = Reim bes reitet) * : "

2 bie oberfiten gegemiber; bie 23 urgel officinale, 23allo wour. Enotig; bie Slume gelb (WOLn. 2luen. 3lb3. 2(pril, M(a)) * - tuberofum, Enot. lige.

Syringa, Jlieber.

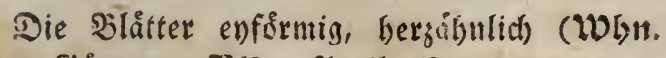

Gårten. Zlbj. 2(pril, Ray) * = vulgaris, fpani. icl)er Şollunder.
T. Tama- 


\section{T.}

Tamarix, Tamarišle.

Die Silưthen romånnig (WWhn. Geffabe. Slbj. Juli ${ }^{*} \quad$ - g e rmanic $a_{\text {beutiche. }}$

\section{Tanacetum, Sifeinfaren.}

Die $\mathfrak{B l i f t t e r}$ boppelt gefietert; bie lesten Etůcfe zerfd)nitten, făgezähnig (WDLn. an Etrafien. 23lbj. Eommer)* =

vulgare, gemeir net.

\section{Taraxacum, Siofhrleinfraut.}

a Sie untern Selddichuppen zurundfébogen; Der Edjaft einblútbig, rơbrig, gang naft

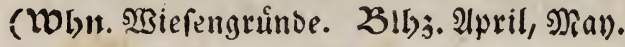
Gebr. Den Sienen, $\mathfrak{A} r \mathfrak{r} n e \mathfrak{y})$ *

$b$ bie Seldsforuppen aifle aufgeridfet; ber vulgare, gemeir nes.

Echaft roftrig, einbluttbig, ganz nalt

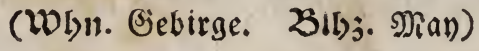
- erectum, aufreds: te?.

\section{Taxus; Eibe.}

Die bllitter auf 2 Seiten genåbert (Woynt. walbidfte Şerge. ZJlbs. fruibling. Gebr.

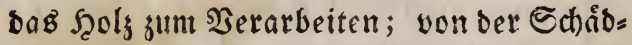

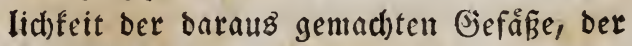
Deerell, und fogar yon der Tóbtlidfét feimes Edhattens ift man nod) immer zweifelbaft)

\section{Bierzafn.}

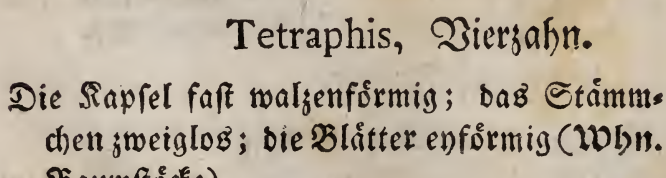

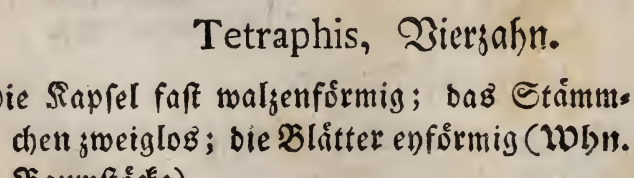
Saumftóde)

baccata, gemeine.

pellucida, ourtis: (ibeinenoer. 


\section{Técírium, Bamander.}

aie Sratter vielfpaltig; die blithenftiele

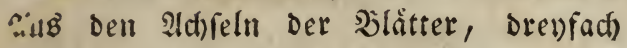

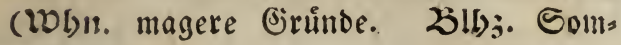
mer)* " = "

b bie Slatter brenfpaltig; die Etůcfe linient: formig; Die Sblutthen einseln, fulrjer als Die Slitter; Der Stengel weitidhetifig; bie field)zånne unbewebrt (LOLn. Hecter. 3!l). 2lıg. Gept. Gebr. Den Färbern, Ed)affutter)

c bie Blitter rägegännig,

I berffirmig; Die Şlütbentrauben eitr. feitig; Der Stengel aufredt) (Whyl. fteinige Gründe. 2015). Ang. Gebr. ben Fårbern)

2 länglid)t, fitiellos; zwo Bluthen in jes

Botrys, Trauben: gamander.

Chamaepythys, Eitopin.

Scorodonia, ef: rooonie. Der Adbfel, geftielt; Der Stengel 4 fant= tig, weitfdrweifig (WOLn. feud)te şBies

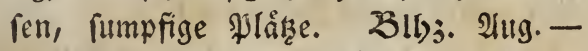
万ૃerbft. Gebr. Den Fråtbern) *

3 Feilifinlich, evforrmig, geftielt; bie Såges g̊ăne tief; bie blutbenguirlen geftielt; bie Etengel belaart (Whin. Fteinigte, trocfue Serge uns Srigel. 25lb). Som= mer) ${ }^{*}$ " = Chamaedrys, ges

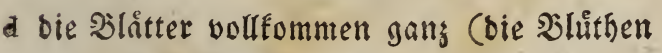
an Der Spifze gedraingt)

I linienformig, unten von anliegenden Seibenbaaren weißß; bie \$lutthen bils Den eimen flad)en Straur (WDLn. Sand

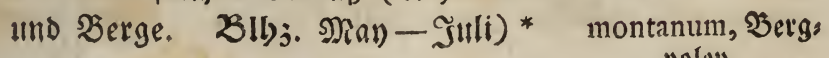
poley.

2 idfmal 
2 fhimal langetformig, unten feint Filsigi oben fein bebaart; benderfeitz grún; bie Rlutben bilben ein äbrenförmiges S.iuptchen (Wolnn. Felien. 25lb). 2lug. Sept.)

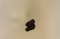

- fupinum, Eleinet Sergpolev.

\section{Thaliktrum, รhaliftrum.}

a Die Slåtter brenfact) gefiebert;

I Die lesten \$låttchen 3tbeilig; die Theile sifaltig, bie গånber gefârbt (WDbn.

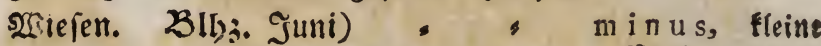

2 bie Slittchen fumpf 3lappig; bie Gaa. Wiejentaute.

men 3 tantig (WDln. Aluen, an 2 segen. 23lbj. Juni. Gebr. Farbe, Den Sies nen) *

b bie 5 latter boppelt gefiebert,

aquilegifolium, nglevblátteriges.

I bie Flittchen 3lappig, fpitig; ber Sten. gel blätterig, gefurcht; bie Siipe an.

- rehnlich, aufrecht (LObn. 2 sicien. Z1!)3. Juni-2ug. Gebr. Den Fárbern, Den รienen)

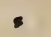

2 Die Slottct)en ganz, und lamsetformig, uno 3ipaltig mit langetformigen Stư

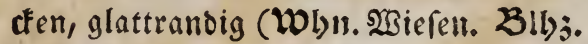
(ommer)*

\section{Thefium, sbefium.}

a Die Blätter linienfórmig,

I langetåbnlich; bie Blütbentrauben mit mebrblůtbigen જ̧fútbenftielen, bie lån. ger find als ibr 2ld) felblatt (Woun. (en. 2゙!bj. Juni, Juli)*

linifolium, lein. blàtterigez.

2 bie 
2 bie Sluthentraube aus einthitfigett, einfachen Eurzen Sbluttbenftielen (DObus. Bebirge. 23lb3. Juni, Juli) = alpinum, 2(tpen

b bie Slâtter lanzetfơrnig, 3nervig; Die Aefte thefium. rutbenformig; bie blúthen in einer siipe (20hn. 25iejen. Sllyj. Juni, Juli) bavarum, Eaiete fojes.

\section{Thlafpi, Scirtentafde.}

a- Die Şlatter långlicht, ge .jut, glatt; die Schottchen tellerformig, serticft, breit ges randet (WDln. Necter 25!bj. NayIuli) *

b bie Blåtter langetågnlich linienformig, ets arvenfe, ?fiferbir: tentafole. was fleifhig, frumpf; bie Edboittchen faft napfånnlid), freizrund (WDbn. Sandgruinde. 23lb). Frůbling) *

c bie Stengelblátter pfeilåbulich, faxatile, etcins birtenta(d)e.

I etwas gefrangt, aufrect); Der Ctengel faft einfach, etwaz bebaart; bie Echoitt. chen oben zufammgebridft, fpariam bes baart (MObn. 2lecfer. S1bj. Frůbling)

2 umfaffend, glatt, gezåbrit; bie șiurzel. campeftre, Feros birtentajoje. blâtter enformig, seftielt, gans; bie Shlumen groffer als sie Relche (Wolnt. magere (jrůnde. 25lbj. Frůbling) * montanum, Serga

d bie Stengelblätter berzformig, glatt, faft Durchroad)sblatterig; bie Echottchen läng. lid)t linfenformig birtentajoje.

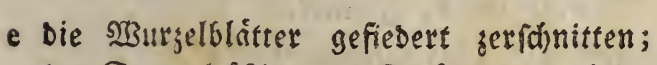
ber Etengel âftig; bie હcho̊ttchen veríebrt bersformig (10lur. Aeder, an Straffer. Żlbj. Ipril - Serbft) *

Burfa paftoris, ge: meine. 


\section{Thymus, Quendel.}

a Die enförmigen $\mathfrak{b l a ̊ t t e r ~ a m ~ G i r u n d e ~ g e s ~}$ framst; Die Stengel niederliegend (WDbn. trodêne Sirinde. 215;. Junt-Sept. (Gebr. Den Sienen, Ranpfer) *

Serpyllum, Felo: quender.

b Die enfórnigen $\mathfrak{b l a ̊ t t e r ~ a m ~ \supseteqq l a t t f t i e l e ~ b ~ e r a b s ~}$

laufend (bie Duirlen ofngefihr $6 b^{\prime}$ 'tuthig)

I vertieft, fehe menige; bie $\mathfrak{B l}$ (v) me reidit tiber ben fieldh zrweninal fo meit her. wor, als er lang ift (Wols.. uno 2 ll);. wie die vorige $\mathfrak{A}(\mathfrak{t}) *$

2 fpiţig, bie Etengel aufted)t; bie Sllume alpinus, 2flpen. quendel.

tagt ůber ben field f.aum fo weit her. vor, als biefer lang i,ft (WDln. u. 23l);. wie oben) *

\section{'Tilia, sinde.}

a Die bersformigen, sugefpijten blatter grof, bellgrůn, fågequignig, etwas raub; bie Jrücte vierfächerig, etwas raub) (Lobn. an Den Strafien ze. 23!hj. Juni, Juli.

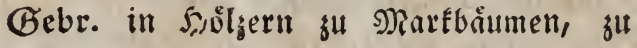
Rauben, Den Sjienen; Sel alts ben Saas men; Den Silobauern; Neißftoblen) * europaea, grof́

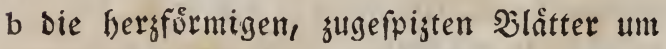
b! âtterige. bie J̧álfte fleiner, etwas Dunfler, ungleid) fägezăbnig; Die Frtichte 5 fåcherig, filsig (Bll);. Juli. Gebr. das frolg ift fefter als bas yon ber vorigen Art)

\section{Tordylium, Birmet.}

Die Slåtter lanzetfórmig, lang gesogen, gefies Dert zertheilt; bie Slattchen gefiedert eins gefdinitten; bie Dolden vielftralig (Wolnn.

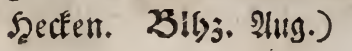

- Anthrifcus, ge. meiner. 
Tozzia, Sojzic.

Die Bltme gelb; bie Unterlippe roth punf: tirt (Wonn. Bebirge. Jlly. Jumi, Juli) alpina, 2l(pen.

Tragopogon, Soct'sbart. tozjic.

Die Reldye fo lang alis bie Slutthe; Die Slatter gan!, aufred)t (Wohn. WBiefen. 21h). Nay, Juni. Gebr. Salat, den \$ienen) *

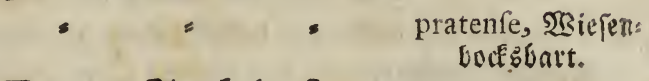

Trapa, Stachelnuß.

Die NRiffe mit vier hornfơrmigen Stacheln

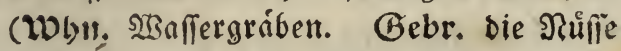
mandelartig, meblecid) )
Tremella, Siallerte.

a Sraniengelf, aufitizend, boutig, faltig (er:

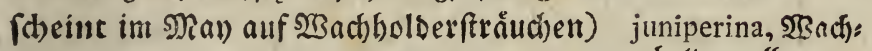

b fohmuticig oraniengelbe, fiellofe, etwas boldergallerte. unregelmáfige Riugeld)en (erfheiurn im

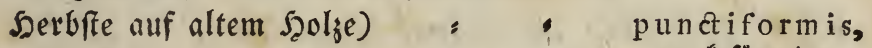

c. gelb, flebrig, in Falten zufammengelegt, punftformige. ungeftielt (erfideint fpait im faerbfte auf alten berinbeten Baunftecfeil) - mefenteriformis,

d femifchleberfårbig, im Mittel auffiţend, getro̊zartige. blattåbulich, am breiten Siande frey, weit. faltig, breitlappig (Whln. faule Stơffe) umbilicata, gena

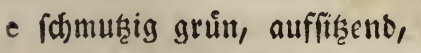
belte.

I wellenformig, båntig, faltig (erfochent. zur Riegenseit an ben Straffen, auf Sainen unb Sisiefen) " Noftoc, Noftock.

2 napfóbnlich) (erid)eint am Fufie ber (j)es buifhe zwifhen ben Noofen auf grobs fanbigem Soben fpât im Szerbife)

orbicularis, napfo ályntidje.

f $\{$ d $)$ mus 
f fohmusig gelbgkử, fuglig, wurgeltteifent (Whn. an ủberichromenten

g fohmars

globofa, tugelforts mige.

I unorbentlich fleinfraus, auffitend, ofne

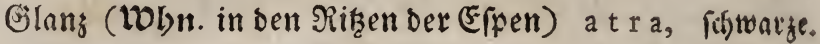

2 abfarbent, geftielt; ber Scut etwas ges mollot, Elebrig (Woln. auf alten \$uthens

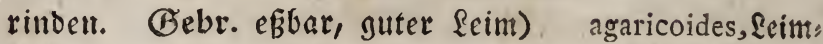

he fommutig weildhemroth, frugformig, gerellig, tremelle.

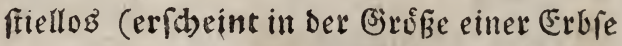
im Eept. alf faulenden Stócfen mad) Re= gemmetter)

it rothe, ineinander verfloffene, im Srodnen lilacea, ritaḱfáis bige. zerreiffende şergen (Whun. auf Baunt: rinden)

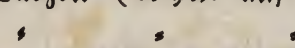

miliaria, Gdja:

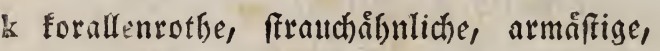
fumpie fiurper (Woln. auf ben sinben alter Sånute)

\section{Trichoftomum, Şaarmund.}

(a d) fle détentes melle.

coralloides, fornts lenrothe.

a Faft ftengellog; bie Blätter lanzetfơrmig, aufred)t; Die Siappe baudbig, langfduab. lig; Die Siapfel elformig malsennéfnlid); Dev Decfel ftumpf fegelformig, etwas fchief (Die Sapfeln fommen im April) pallidum, blaffer.

b Die Slåtter fảmal langetformig, aufred)t; Die Rapfelftiele mit einem ftumpfolitteri= gen Sield)e, aub. Dem Grunde ber 2leftchen; ber Decfel Eegelfórmig, etwas fhief (Die Sapfeln erfheinell im 2 (pril) $\quad$ bafiflorum, glunto.

c Die Borften Der Rapfelmůnoung gegliebert; blůthiget. Die Siapfel faft walzenformig, gebogen; Der Decfel fegelformig ; die Reapfelftiele gewun=

- Den (erfacinen in Den $23 a$ loungen im May) tortum, gemundes net. 
d bie Ståmmdben grweigig, nieberliegent; bie Sblätter fabnfơrmig lanzetåbnlich, in ein meiñes haar aแs่laufend; die Rapfelftiele faum ro lang als bie anveige (Whn. 235 al. Dungen)

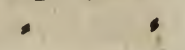

bie 2 efte fabena

e Die Strimme friectend; bie fôrmig, aufred)t; bie Slätter nervenlog, fpisig langetformig, wegftebend; bie Sias piel in Der Itrgend gerabe, Dann frumm; bie Deckel fumpi

\section{Trientalis, Echimfraut.}

Die Blåtter eyfortmig lanjetảhnlich) (WDhn. Noorgrund. 2 3 !bj. Juni) , = europaea, euto. Trifolium, Silee.

I. Der Stengel friectient;

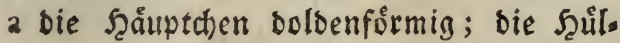
fen vierfaamig (WObn. SWiejen, Sweis Den. 23Il)3. Nan, Juni) * - repens, ftichen;

b bie Scaiuptchen faft fugelformig; die repens, flichen: Det.

hypnoides, aft: moosáfnlidjet.$$
\text { paijches. }
$$

Relche an ber Seite ber Fabne frart

behaart, nach bem

rhmollen; die Sllume blaß̧roth (Wolnn.

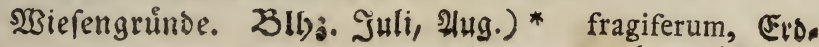

II. Der Stengel aufredt,

beerentlee.

2 meitfhneifig,

x Die 2lebren etwas zottig, zwifichen żwen ftiellofen (jegenblăttern; Die Sllumen einblätterig; bie SBlåttchen faft tellerformig (WOLn. STBiefen.

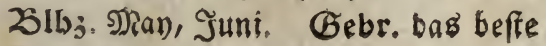
Futterkraut) * 
2 sie 2 efiren faft fugelformig, mit ges orångten raufhenden 3 hlithen; bie Fabnen abruirts gebogen, bleibeno; Die Slattanfiţe berffôrmig, gefran. zet; Das ungleiche Sblattthen beutlid geftielt; Die Shlume gologelb; bie ganje Sllange fehr flein (rolyn. 2 es dFer. 23llyj. Juli- Sept. Gebr. Futtextraut) * = : =

3 Die Alebren jottig, lang; bie Slumen agrarium, 2ffect; einblatterig; Die Reldhe glatt; Die Reldbjübue bebart; bie Silattchen langetformia, fein fägezáfnig; ber Stengel, wemn er trocknet, boljhart (Wolr. an Etraffen. Slly. Juli. Gebr. Futterfratit) = . rubens, tóthlidjer.

b am Grumbe niebergeworfen, boh, bicht;

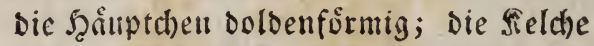

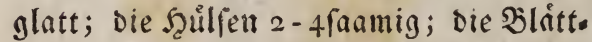
d)en enformig lanjetínnlid) (LObn. SRies retr. 23!bj. Juli. Gebr. vortefliches 2ielbfutter) *

c fiemlid) einfacty,

hybridum, lang: blátteriget.

I faft runtidt), f(t)neibig; bie blutthen. hăuptchen fugelformig, jottig, jebes von einen Sblutthenblatte geftuktzt; bie Slittchen langetfơrmig, fein fägezăh = nig (Whlt. Bllyj. unb Gebr. wie Die vorige $2(r t)$.

2 bie $\mathfrak{A}$ ebren enfơrmig, meifteng oren, alpeftre, 2ilpens mit georångten Slüthen; bie Fabne Iang lanjetformig; Die Sieldhe glatt;

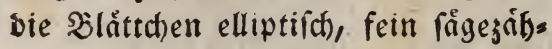
nig; die Bllume weiß ( $\mathfrak{W}$ l)tr. magere

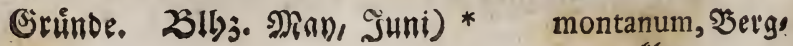
tlee. flee. 
3 Die Aebren einfad), mit gebringten rallichenden Sblütben; bie Sieldye glatt; die Slattanfáţe lanjetfơrnig; Das ungleidhe Slättchen faum geftielt

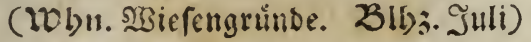

aureum, golofít: biget.

d armblứthig, oben nebft ben fieldien bes baart; Die 2lebren enformig, mit ges

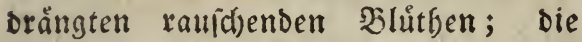
Fafne bleibeno; bie Blume gologelb) fpadiceum, farmes

e brevfantig, gablicht, an ben seriftun= litfärbiget. gen abgebogen; bie Dlütbenbåuptchen fuglicht, bebaart, blattlos; die Slátt=

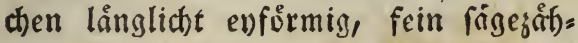
nig (WObn. 21l)j. und Gebr. wie die anbern guten Arten)

flexuofum, abges bogenet.

III. Die Slumenaihren evrund, zottig; Die 3ähne ber Slumendecke haarig; Die Sllu= mendecte fo grof als bie Slumentronen; bie fluigel ber $\mathfrak{B l u m e n f r o n e n ~ i n m e n d i g ~}$ mit einem rothen Fled"e (WOLn. Hedfer, Raine. Slbj. Sommer)*

arvenfe, Sakgens tlee.

\section{Triglochin, Triglodjin.}

Die Rapfel 3fåcherig, lintenformig (Wogn. Eumpfe. 25lb;. Juli)

Triticum, NBeif̧en.

paluftre, Eumpf trigloditin.

2 Die Relche vierblutthig, am Girunbe etwas bebaart (Słl)j. Juni, Juli)* " cereale, gemeiner.

b bie Relche vierblutbig; bie benben frucht. baren Slưthd)en mit Granmen; bie Gaas men von ben Seldhtlappen eingeichloffen;

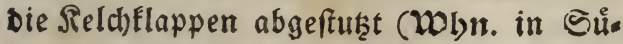
ben und Submeften oon Saiern. 25lbj. Juni, Juli)

Spelta, Dưnter. 
-c bie 2 eblethen zufammengebridft, 4-66he thig; bie Sielche fdumal, foighig; ein Iheil Des Şalmz unter ber Erbe friectient, fChuppig, murzelågnlich) (WDbu. Gånten, ล3tutne. 23!b3. Juni, Juli. Gebr. 2ieb= futter, su Rajendecfeu) *

repens, 2uecfe.

\section{Trollius, Srollblume.}

Die Blume Eugelformig, balbgefoloffen

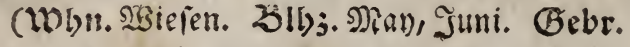
Den ミienen)

\section{Tulipa, Sulpe.}

europaeus, Engets blume.

a Die Stanbtriget pfriemenformig, butchs nus glatt; Die Sbluthen "aufredt); Die Slatter breit lanzetformig * - gefneriana, Bants

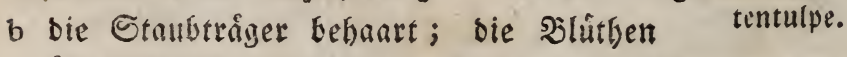
uverbanticno

\section{". " \\ Turritis, ₹lyurnfraut.}

fylveftre, frúfe.

a Dic Slatter alle fteifborftig; bie untern Etengelblítter enformig, bie mittlern lång. lidf, bie obern ictmal; umfaffen alle bent

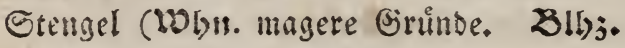

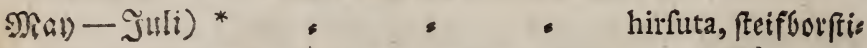

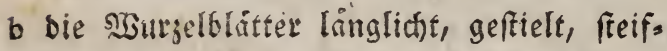
geb. borftig; bie Stengelblätter unfañend, glatt, Ianjetåfulid), pfeilformig; die Blütben flein, gelblicht weif; die ganze \$flamşe blaus

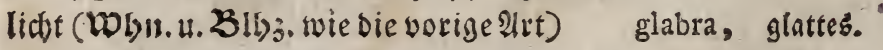

\section{Tuffilago, Şuñattid\%.}

2. Der Scraft einblütbig,

I faft naft, Die Slätter tellerformig, ges

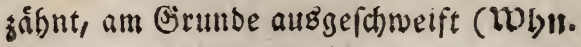
(s) birge. 25!43. Suli)

alpina, 2r(penthufs lattiti). 
2 Beiduppet; bie Blatter tellevifnnlid) berjfờrmig; bie weiblichen Sluthdhen mit Şalbblund)en, febr zaflreid) (WDlyn. Tbongruinde. 23lbj. Frubling. 'Gebr. ben ふienen; tun ?lotbfalle zur Epeife) * Farfara,gemeinet.

b ber Slithenbuns fitrausfoúrmig,

I bie weiblid)en જlůthdhen bes $11 m$ freifes

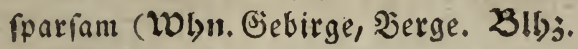
Fruibling) *

2 bie meiblidben \$llutbd)en zablreich, mit alba, weißBe $\mathfrak{P e f t i s}$ einem Blumenrande; bie \$lâtter brens. ectig, berjformig, gejo̊nnt; bie f̧er: lappen buctitig (WWhu. Wicengrübe. 215). รrưbling) - Spuria, falide 2 es

c ber Slůthenbund evfoormig; bie ßhlithen ftilenfmuiz. alle Zwitter; Die, Slátter berfförmig (WDbn.

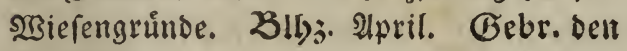
ઝienen)

d bie Shluthentraube lang; bie Etiele ein. Lenflouty. blüthig; bie Şlitter tellerfórmig, am Siande gezåbnt, am Orrunde berzformig ausgeidrueift; Der Echaft fduppig; zabls reidhe weiblid)e Sluttbden obne Şlumen:

- ranbe im umfreife (WDhn. feudte 23 ies

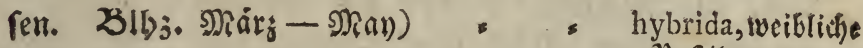

\section{Typha, \$Noosfolben.}

Peffilengroury.

2. Die månlidien und " weiblichen Sluttyen genciijert; bie blatter faft Degenformig

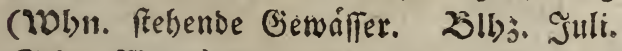
(Bebr. $23 \mathrm{atte}) *$

- Latifolia, beteits

b bie månnlichen sluethen voli ben weiblis bláttertiget. d)en abgefonbert; bie Slätter balbrunb (20lon. Grąben) *

ang u it ifolia, idgmalslatteriget.

U. Ulmus, 


\section{U.}

\section{Ulmus, HIme.}

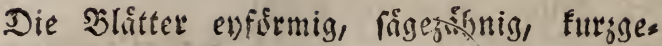
frielt, an Grunbe ungleich bith angefertst (20 h). 2tuen. Z3lb3. Frůlling. Gebr.

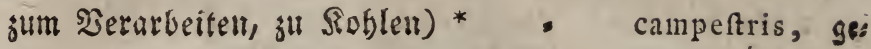
meine.

\section{Ulva, Lltoe.}

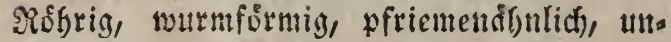

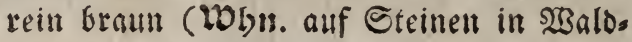
bádjen)

\section{Urtica, SReffer.}

vermicularis, wurmformige.

a Die Slåtter evforrnig, gegenůber

I faft vollommen gang; Die Frucbtgapfent Flugelformig (Wohn. Sintrten) = Dodartii, Sobat:

2 grob figgegrifnig; bie siąchell walsells tilicise.

fermig; bende Befhledter in Einem (10hu. Gartenlano. 25lbj. Sommer. (Gebr. (jemůs) *

b bie Gegenblitter bersfơntilg, grob fågejåb) urens, fleine siennneffel. nig; Die Bltitgen 2 båntg; Die Trauben gepaart (Wonn. allentbalben. 26lbs. Coms ner. Gebr. Giemis, zu Bontn uno Suth,

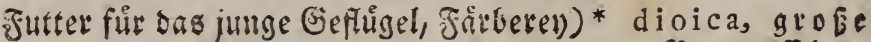
Sitennneftel.

\section{Ufnea, Nixhaar.}

2 Ėaumichwars, felbe neftig; fefre lang, glatt, wurgelforrmig, inmendig futrammig, filjig (WOLn. in 23 afferleitungen, zwifhen Rinde

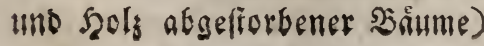

radiciformis, 10ut: zelformiges.

$b$ idjwars 
b (d)toary

I ziemlid) cinfach, pferbbaaråbnlid) (IDb)t. zwifchen abgefallenen פiabelı ber פใa. Delfoslzer)

$=$

2 fraud)afbulid), bart (IDbrt. auf Felfen, Die fidh mit Einter luberjieben)

c meifie, faum åftige, felfr lange, burchein. hippotrichioides, pferobaatformi: faxofa, feliens nixbani. nnder gefolungene Fäben (20hir. im Sies funobade zu Adelbolsen)

,

alba, weí̉es.

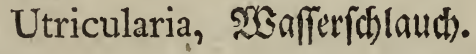

a Der Sુlumenfporn fegelformig; Die 2jlůs thenfitiele foluppig; bie Slatter franzens

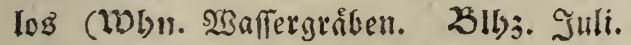
Gebr. Farberey) *

b ber Slumenfporn mit eitren Siele; bie vulgaris, gemeis ntet.

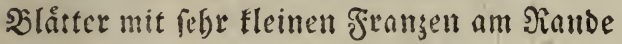

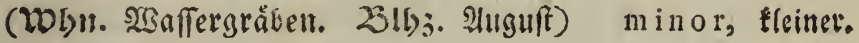

\section{Uvularia, Båkfleinfrout.}

Die Sblâtter umfaffens (Mobn. Sisfmerwald. 3ll). Juni. Gebr. Die sisurgel zum Ealat)

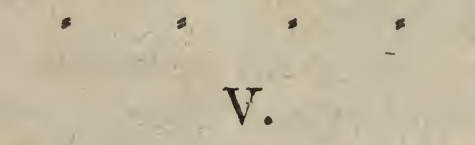

amplexifolia, ums falienoer.

\section{Vaccinium, Sacibelbeeren.}

I. Die Slâtter enfírnig,

a volfommen gang,

I meiftens am Grunte gefrant; sie Sllitbenfiele einblütbia (WWli. Mios raffe. Zlbj. May, Juni. Gebr. Ju כrandtwein, ben Fintbern, ben

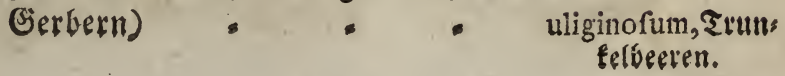


2 an Ranbe umgerolft; ser Etengel friechend, fabenfornig, unbewchet (Whn. Moraifte. Blly. Juni. Geb:. Thee, bie beeren enthalten eine un. rdhóbliche Sitronenfúure)

$O x y \operatorname{coc} 0 s_{2}$ Sivosbeeret.

b făgesăhnig, abfallenb; ber Etengel aftig;

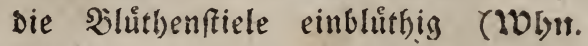
Traibungen. Sllyj. Ilpril, গiay. Gebr. Speife, Den Serbern, Jäberey, Brandt. wein, ben \$ienen)

Myrtillus, ges meine.

II. Die Slitter verfebrt eyforrnig, an Sanbe Juruckgebogen, auf Der unterfeite punftirt; bie Irauben an ben Epitzen Der âtweige

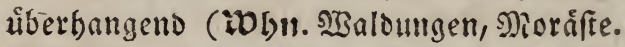
23ilj. Soumer, f̧erbft. Gebr. Apotbede, (jerberey, (jallerte, Srandtwein, Ibee)

$V$, it is idaca, soreubetseren.

\section{Valeriana, ふaalorian.}

I. Sie Sbluthen 2bainfig; bie obern Slaitter gefiebert (Wonn. jeudte 2 siepen. 25!bs. \$ay, Jumi) *

2 Die şlôtter alle gefiebert (SOLn. umb Blb3. wie Der vorige) *, = oficinalis, gemeis

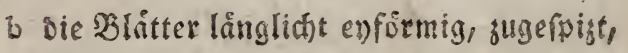
dioica, flimet Oumpfoaloriar.

- faum gezånnt; Der Stengel einfact (rogn. Serggegender. 25lb3. JumiSept.) *

montana, Bers balovian.

$\varepsilon$ bie Slåtter långliç)t, reitlåuftig ge. net. Jáhut; bie Caamen einfad); Der Etell. sel gablicht (WDbla. Ilckerraine, Şálle. 231b3. Nan, Juni. (Gebr. Jeloralat)* olitoria, Ealats balorian. 


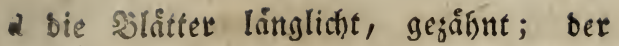
Eanme 3zåfnig; ber Êtengel gablicht

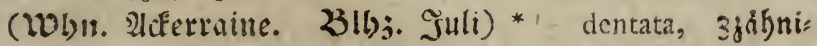

c bie untern \$lătter langgeftielt, bie oben sfingerig (WVhn. Alpen. Slh). Juli) tripteris, zfinger

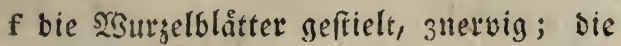
riget.

Etengelblätter linienforrnitg, aufitisend, flein (Woln. Gebirge.' 3̇lbj. Juni, Juli)

\section{Valantia, Zalantie.}

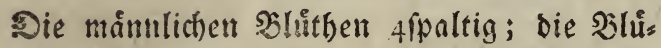
thenftiele zblåtterig; bie Slätter enfiors.

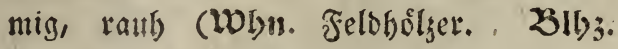
Juni) * ,

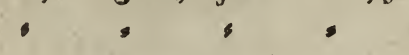

\section{Veratrum, Siermer.}

Die Traube regk zufanmengéfegt; bie $\mathfrak{B l h t}$ menblåtter alfred)t (WObn. Alpen. Blbj. Suni, Sult. Gebr. Die Șsurgel, gepuls vert und mit Miebl vermifd)t, giebt eirt gutes Mafffutter für bas (Beflugel)

Verbafcum, MBollfraut.

A. Die Dlatter benberfeits filgig, berablaus fens,

I ber Stemgel cinfad),

a bie গlarbe zwentuglicht (Wobn. an Etraffen. Slly. Juli, 2lug.)* bicolle, 2narbige

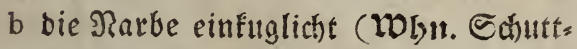
אisnigzferge. baufen, Etraffen. 23lb3. Juli, Mtug. Gebr. sum Shee, den Bienen, fait: beren) *

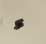

Thapfus, Şims
melškelye. 


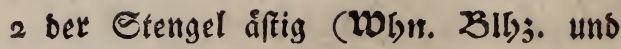
Gebr. wie bie vorige $2 \mathfrak{n t})^{*}$. Thapfoides, iffige

B. bie ஒlåtter unten weißlid)t, filsig, langs Roningterze. gegogen, enformig, fpitig (Wobn. Båume, Şecter. Silj;. Juli-Eept. Gebr. Den Sienen) *

s.

Lychnitis, fleine

C. Die Slätter gekerbt, eyformig, glatt; bie Ronigbetige. Stengel faft Glattlog, einfach; bie ein. fache Sllunentraube am Ende; bie Slle then bunfelroth (Woln. magere Grůnde. 23lbj. Juni, Juli) *

-

D. Die Slåtter boppelt geferbt, långlidft bergformig, geftielt; bie gelben silumen am Schlumbe mit veilchenrothen flecten; bie Triger mit veilchenrothen Saaren bes reģt (WWlir. an Giråben. Żlyj. Suni, Juli. Gebr. Den Sienen)

E. Die Slätter faft boppelt fágegännig, um, fallent, langetformig, glatt; Die Traube an ber Epitze, furz; bie Slutthenftiele einfach (Wonn. Straflen, Raine. 25lbj. Suli, 2(uguf)

.

Blattaria, gel6es
Diottenflaut. phoeniceum, roths braunes 9)ottens kiaut.

nigrum, id)warzes 230 llfterat.

\section{Verbena, Eifenfraut.}

Die 2ebren fabenfơrmig, gerifpet; bie Slåts ter vielfach in Rappen zerichnitten; Der

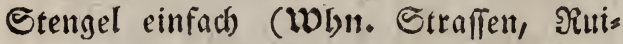

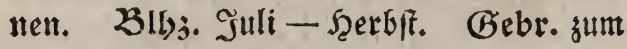
(Einmadben ber (jurfen) * $\quad$ = officinalis, get wosbulidjez.

Veronica, Efrenpreis.

I. Die Bhítbentrauben aus den Eeiten,

2 bie Slåtter enfơrmig, 
I geferbt, gesemiber; ber Stenget niederliegent; bie sibenabuliden

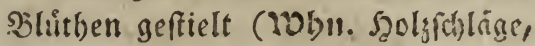
¿erge. Blly. Jutmi. Gebr. Die Blåtter zum Sbee) * = s oflicinalis, ges

2 flach, gegemiber; Der Etengel fries wóbulitlet.

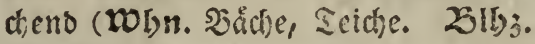
(ommer) *

- Beccabunga,

3 runglicht, geşâbut, friellos; bet כ̧adjounge. Stengel auf zmo Eetten bebart (W0bn. NBiefengrunde. 25il);. Na), Jัmi)*

-

Chamaedrys, ga• manderblitteri:

4 Innetrignlich); Der Etengel aufrecht get. (WOLn. 3erge. 23lb). Nay - Juni)

b bie Şlåtter langetfórmig, urticaefolia, nê Fefblatteriget.

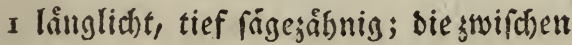
Den Siluthentrallbelt linienformig, zalinlog (WWhn. Sisterengrůnde.

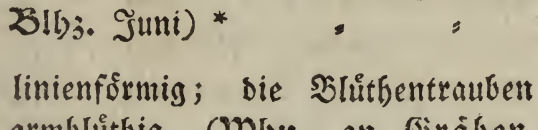
armblutthig (Wobu. an Biraben. 3!bj. Эu(i) *

auftriaca, sftels reidififiser.

3 ber Etengel aufred)t (WObur. an fies

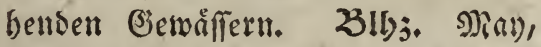
Suni)

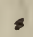

Anagallis, $23 a i n e t s$

II. Die Slůthentrauben nus ben Idffeln, gaucbitil. lang, armblutthig; bie Slatter evformig, geferbt, geftielt, fammt bem nieberliegens ben Stengel bebaart (Mbn. WSaldum, gen)

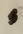

montana, Vergs eflenpletis. 
III. Die Sluthentrauben nabe an ber Spitse Der Pflanze, fefre lang; die blatter eys. fơrmig, gegenuiber, gegånnt, rumglid)t, ziemlich) ftumpf (WObn. WBiefengrunde, Şe

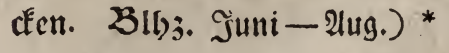

Teucrium, כetgs batbengel.

IV. Ein Slüthenbuichel an ber Spitze; bie Şlâtter bes Fruct)tfengels länglicht ens. foermig, gegenúber; bie an ben unfrudgts baren Aeften bidjter uno fleiner; Der Stengel frrauchartig (Woln. Gebirge. 23lij;. Eonmer)

V. Dex Siluthenbuifhel an ber Spise bes - fruticulofa, fauls viget. obern blattloren Schafteg, flein; bie Blåt. ter am Grunde eyforrmig (Wobn. Eable Gebirge. Sllyj. Iuni, Suli) - aphylia, blattiofet:

VI. Die Bhlithen fparfam an ber Epiz̨e; Die Sisurgel friectend; ber Stengel eins fach), unten nieberliegend; bie Bjegen. bläter Eurs geftielt, rundlicht evfoirmig, feicht geferbt (Wolnn. Alpen. Zlly. Juni, Julii)

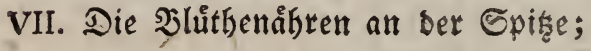

alpina, Ripen efrenpteiz.

a bie Sllitter fågę̧ånigy,

I nit abwech) felnben gro̊kera und fleis nern 3åthen, meiftens breyfach, lang= gejogen langetåthlich (WVlni. magere

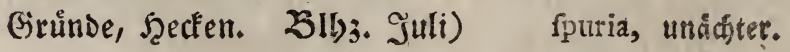

2 ไangetfơrmig, fpişig, gegenu์ber (WDLn.

2siefengruinde, fecten. Sil5j. Suni) longifolia, langs blátteriget. 
b Die Blätter geferbt, ftumpf, gegenüber; Der Etengel volffommen einfad), aufs ftebeno (10Ln. wie die vorige. 23!ly;. ذuแ⿰ - 2(ug.) *

-

c bie Slåtter eväbnlidh, glatt, gegemúber; fpicata, åhrentrus genoet.

Der Stengel murgelichlagent ; Die $\mathfrak{S l}$ lus the weišlicht (WDin. magere Grtunde. 3l5j, Nay, Juni)* . . ferpyllifolia, VIII. Die Slůtben cimgeln; quendelblátteriget.

a sie $\mathfrak{W l a ̊ i t e r ~ h e r s f o r m i g , ~}$

I geferbt,

a linger als bie shluthenfticle; bie

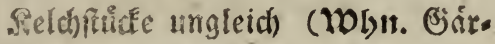
ten; Ilecfer. 25!l3. April Set乡fif) * , , , - arvenfis, 2fdeti

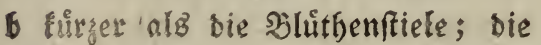
ebrenpteiz. sielchifurde gleid) (WObn. Alders lans. 23lbj. April, Naß)* agreftis, Felo. efrenpleis.

2 bren-firmflappig, flach; bie fappen

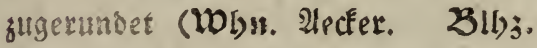
Sipril, Nay. Gebr. ein Futter fur Die (s)afe)

3 oren - funfectig, faft keilfơrmig; bie hederifolia, ephetts blätteriget.

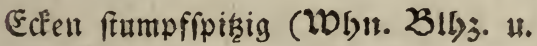
(Gebr. wie bie vorige $2(r t) *$

Lappago, ectotát tetiget.

b bie blätter glatt, gesăgnt, segenůber; ber Stengel etwas befaart, ziemlich nufred)t, fehr flein (IDLn, magere Gruำde. 23!hj. Juni) * = acinifolia, bergs quendelblätteriger. 
c bie untern Blitter 5fingerig; bie obern 3 fingerig zertbeilt; bie bluthenftiele fưrzer als bie Slåtter (WDlsu. Alecter. 2కlbj. Jrrúgling) *

verna, frủher.

d bie Shlutthenblätter sfingerig sertheilt, fưrzer als bie Slúthenftiele (Woln. แ.

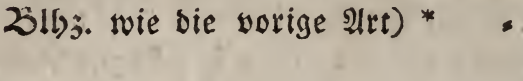

a Die Sllitter enforrmig, fágegannig, aberig,

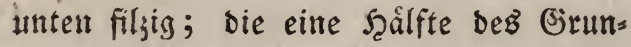
Des etwas hofber (MDhn. J̧ecten, 2lten. 2515;. April-Juni. Gebr. Yogelleim; Den Faß̧Gindern) * = - Lantana, Erthlins

b bie Slatter lappig; bie Eappen edig ges triphyllos, steys blitteriger. jăhnt; Die Slattftiele bruifig (IVIn. Şes čen. Sllyj. Juni. Gebr. Den Bienen; Die \$ceren Den Do̊gelm) *
Vicia, Wricte.

*.Die Shluthen geftielt ;

I. Die Sllutbenfiele vielblůtbig,

A. Lang; bie Slůthen badbjiegelfórmig; Die Slåttchen lanzetfórmig, banrig; Die Şúlien glatt, vielfaamig; bie Slưthenanfäze ganz (DOln. Adfers lant. ZIb). Juni bis 21uguft.

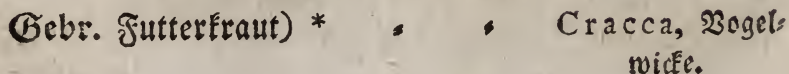

B. bie 
B. Die Blättchen enfơrmig;

a sie untern ftiellos, ant aneige;

Die SBlattfiele vielblátterig, bie

Blumen gelb (WDLu. Idfarland) pisformis, cubfen fortmige.

b mit einer Granne verfeben,

I abgeftorlen frumpf; bie Slatis anfätze gezähnt, auf Der unter. feite mit einem eingebronnten Fledfe (WOLn. Şectien. 25lb). Juni, Suli) * - dumetorum, Şcs

2 långlid)t; bie Slumen geftreift; dentriffe.

Die Scuilfen fâbelformig (WDLn.

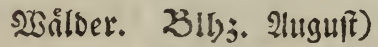

fylvatica, $\mathfrak{Y a} a l_{0}$ twicke.

3 zefin an ber Zabl, etwas ipitzig; bie Slattanfâta pfeilformig, ganz; Die fieldhe behaart

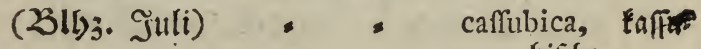

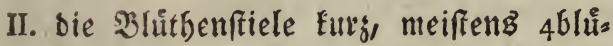
biid)e. this; Die Slättdyen evformig, gegen Die Spize fd)máler, gerumbet, mit einer Gramne, nach Der Ed)linge bin abwad)= feno (WInn. Idferlnno. 25l5j. Juli. Gebr. Sutterf(raut) $* \quad$ : $\quad$ Sepium, 3nun wicfe.

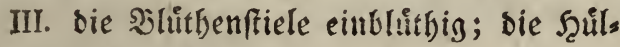
fen aufrecht; bie Sllattchen 2 \$aar, exformis, lanzetríntlid); Die Slattán.

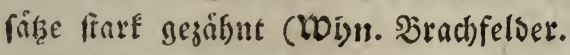
Gebr. Jutterfraut)

bythynica, baiets idje. 
** sie \$lüthen ftiellos ; bie f̧úlfen in bes

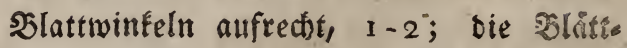
d)en am Enbe abgeftoffen, mit einer fur. zen Eranne; Die Slattanfíze ges̆innt, mit einent eingebranten flecten (Woln. 2lecker. ZJlbj. Juni, Juli. Gebr. Pferb= $\begin{aligned} \text { futter) } * & = \\ & \text { Vinca, Sinngruir. }\end{aligned}$

Die Stengel nieberliegeno; bie Blatter lans setâfulich, evformig; bie Shluthen geftielt (WDIr. STBâlber. 23l5j. Itpril-Juni. Gebr. Cerberlobe) minor, Eleites:

\section{Viola, Deilden.}

A. Der Stengel feblt;

I ofme Ranfen;

a bie Blatter nierenformig; bie $\mathfrak{B}$ lü.

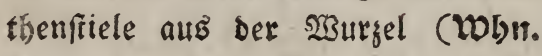
Bebirggegenden. 23lb3. Juni) paluftris, Morafis

$\checkmark$ Die $\mathfrak{b l a ̈ t t e r ~ b e r g f o r m i g , ~ e t w a s ~ r a u b ~}$ fativa, Snaturife. (20511. magere Gründe. $2515 j$. Mårq, 2(pril) * = = hirta, rauber.

2 mit Ranfen (WOIn. Gebrege, Girten. 25!5;. Frůgling) * - odorata, MRázen veilderen.

B. Det Stengel feblt anfangs, wridfit aber veilduen.

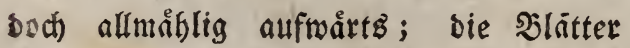

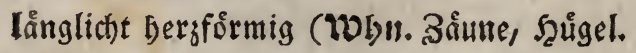
25!bs. 2pril, פ(ay) *

canina, Scunds: veildsien. 


\section{Det Stengel aufrectit,}

I bie Slåtter bersfórmig, lanģetrignlich, fågęånnig; die Sblattanfiase balb lan.

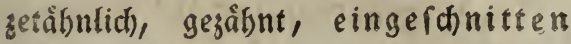
(W01). Derge, magete Siruinde. 31th3. Iuni)

-

montana, Berge. veilden.

2 bie Blåtter verfebrt eyfơrmig, geferbt; sie slattanfáte gefiedert zerfhnitten (Molr. N3iefen, 2lecter. Zlb). May) Detob. (Gebr. Tbee) * - tricolor, Frevjam. finut.

D. Der Stengel meitidneifig, äftig; bie 2Jlatter wed)felsheife, berzfơrmig, finge. Jåknig, fpişig; bie Blưthen langgeftielt, ลนธ่ Den Slattwinfeln

- bavarica, bailio

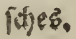

E. Der Etengel orenfeitig; bie Sthteter nierenformig, bergånlid); einige જlle: then blumenlos (DDln. Serge. 231bj. खนเท)

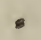

-

mirabilis, fett fa mę.

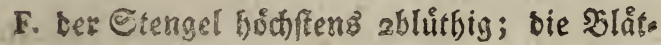

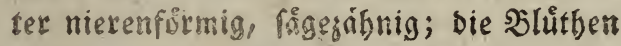
fleiner als Die Sblátter, gelb (WDIn. Be. bitgr. 2316. Juli)

bitiora, 2llpen veildien.

\section{Vifcum, פniftel,}

Die Slåtter frumpf Langetfórmig; bek Ster. gel gablicht; bie blüthen in ben 2thfeln (Whln. auf Raubbaumen. 25l53. Ipril.

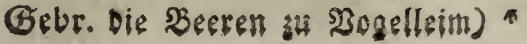

album, neifiet.

Vitis, 


\section{Vitis, NGeinrebe.}

Die Slâtter lappig, budftig, naft (WWInt.

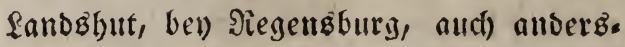
wo in Girinten. 23lyj. Miay, Juni. Gebr. Die abgefd)nittenen Zweige fammt Dem falibe jur $\left.\mathbb{R}_{\mathrm{obgarer}}\right)^{*}$, . vinifera, gemeine.

\section{W.}

Waldfchmidia, IGalofdmibie.

Die Silatter gerunbet, ber fifrernig, wouffont. men ganj; sie Blume gelb (WObn. Ş̧ǻs (er. $i$ Zlbj. Juli $)=$
Webera, NGebere.

nymphoides, Eumpfiwalofitmis Die.

a Die Siapfellt gefturzt,

I eyforrmig, wenn fie fratben ober reif fino, feeben fie aufred)t; die Etrímm. d)en unten armblätterig, mit einem

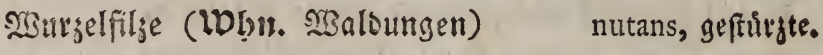

2 faft walgenformig; ber Dectel getwólbt,

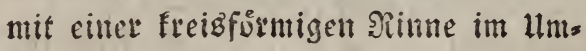
fange; bie kinfern Mindsångne breit, úbereinander liegend; bie innere Şaut franjentog (Wobn. Ssaloungen. Die Shapfeln reifen int 2pril) $\quad$ S Trichodes, bants

b bie Siapich aufred)t, funelforonig; bie blätterige. Sllâtter pfriemenfôrnig (Wobr. sisalbun.

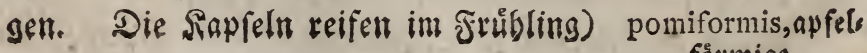
fơrmige. 


\section{Weiffia, গ⿱\zh21}

Siriectend; bie Sweige aufred)t, in zwo fidh Decfenden Jlåchen gefiebert; Die Slått. d)en langetformig, gefpigt, auf ber Dbers flache genellt; Die Siapfeln friellos, aus ber Mitte (WOHn. Saumrinden) - pennata, mefies

\section{X.}

\section{Xanthium, Spifstette.}

Der Stengel unbetwehrt; Die Slätter bers: fórmig, 3nervig (Whn. 3ninne. Zlbj. Juli. Gebr. ber Siern ef̧́ar) - ftrumarium, gei Xylaria, Singetpils.

Echwars,

2 åftig, bolsartig,

I bie Spicen fegelformig (Whn. Șálfe) $=$ clavata, feulfors

\& bie Epiŗen gefingert; bie Finger zufammengebrüft (WWIn. faulende Sretter, alte Stócfe) = digitata, gefinger,

b einfach), fabenforrmig, bas se̊pfchen flls tet. gelfurrmig, mit einem weiflid)ten ঐeife, Der fich wegwifchen lifift (WWhn. alte (Eidjenfto̊cfe) " = . fphaerocephala,

6 bơd)f einfad, pfriemenformig, bet mit tugelfórmiget. Dem Eamen bedecfte Theil gefhlan. gelt geltet.

z. Zanni- 


\section{Z.}

Zannichellia, 3amnicjelie.

Der Stengel fabenformig; bab \&aub borfter. ågnlich, gegenůber; bie Sllutben in bett

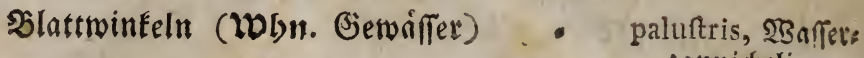
zannidjelie.

\section{Zea, NRans.}

Die Slătter am Sande gan: (Gebr. 9ikebl, Siebfutter)

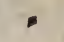

Mays, gemeinel: 




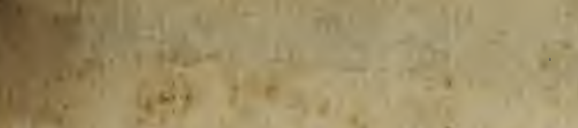

a

\section{2. $13, y=$}

ixty ix

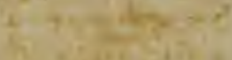

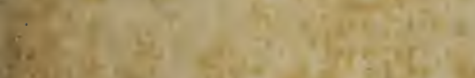

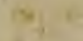
8.
If

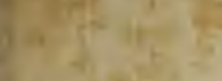
is:
$-7 x^{\circ}$

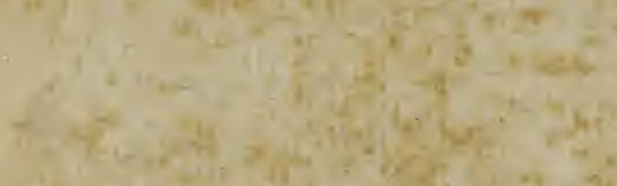

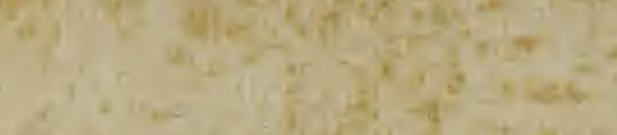

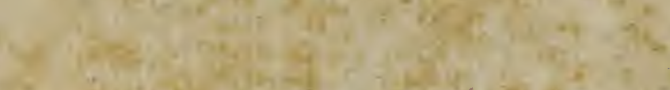
tin

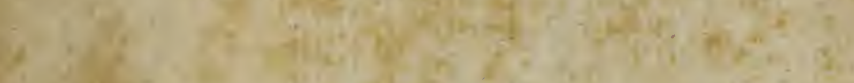

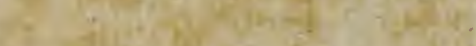

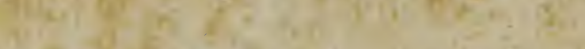

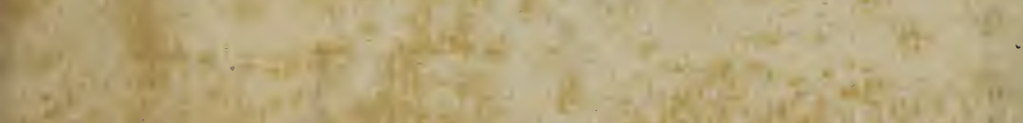

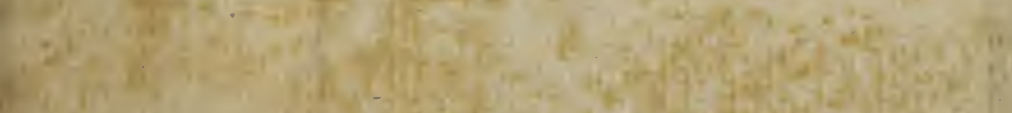

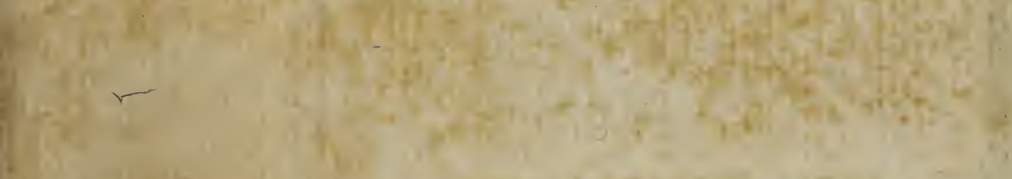

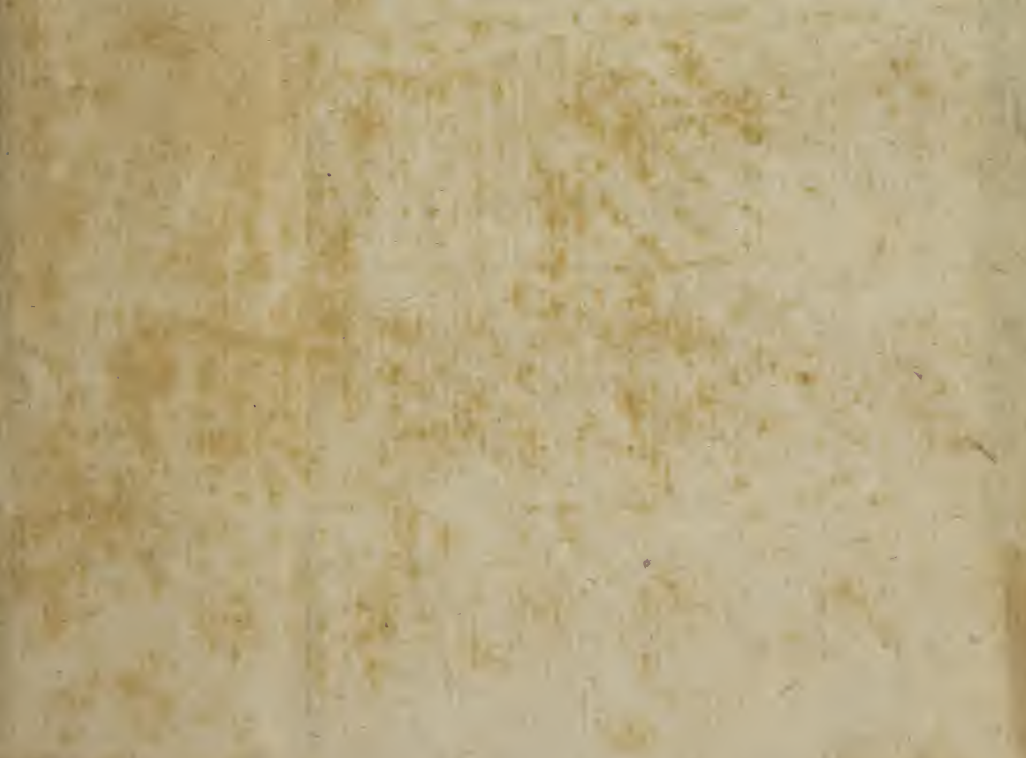


